\title{
PHOTONIC QUASICRYSTALS: \\ A CASE FOR CRYSTAL ANGULAR MOMENTUM \\ by
}

Khaled Mnaymneh

B. Sc., (Windsor), M. Eng. (Carleton)

\begin{abstract}
A Thesis
Submitted to the Faculty of Graduate Studies and Research in Partial Fulfillment of the Requirements for the Degree of Doctor of Philosophy
\end{abstract}

Ottawa-Carleton Institute for Electrical Enginecring Department of Electronics

Carleton University

Ottawa, Canada

Copyright $\odot$ November 2007 by Khaled Mnaymneh 


$\begin{array}{ll}\begin{array}{l}\text { Library and } \\ \text { Archives Canada }\end{array} & \begin{array}{l}\text { Bibliothèque et } \\ \text { Archives Canada }\end{array} \\ \begin{array}{l}\text { Published Heritage } \\ \text { Branch }\end{array} & \begin{array}{l}\text { Direction du } \\ \text { Patrimoine de l'édition }\end{array} \\ \begin{array}{l}\text { 395 Wellington Street } \\ \text { Ottawa ON K1A ON4 } \\ \text { Canada }\end{array} & \begin{array}{l}\text { 395, rue Wellington } \\ \text { Ottawa ON K1A ON4 }\end{array} \\ \text { Canada }\end{array}$

Yourfile Votre référence ISBN: 978-0-494-36792-6

Ourfile Notre référence

ISBN: 978-0-494-36792-6

NOTICE:

The author has granted a nonexclusive license allowing Library and Archives Canada to reproduce, publish, archive, preserve, conserve, communicate to the public by telecommunication or on the Internet, loan, distribute and sell theses worldwide, for commercial or noncommercial purposes, in microform, paper, electronic and/or any other formats.

The author retains copyright ownership and moral rights in this thesis. Neither the thesis nor substantial extracts from it may be printed or otherwise reproduced without the author's permission.
AVIS:

L'auteur a accordé une licence non exclusive permettant à la Bibliothèque et Archives Canada de reproduire, publier, archiver, sauvegarder, conserver, transmettre au public par télécommunication ou par l'Internet, prêter, distribuer et vendre des thèses partout dans le monde, à des fins commerciales ou autres, sur support microforme, papier, électronique et/ou autres formats.

L'auteur conserve la propriété du droit d'auteur et des droits moraux qui protège cette thèse. $\mathrm{Ni}$ la thèse ni des extraits substantiels de celle-ci ne doivent être imprimés ou autrement reproduits sans son autorisation.
In compliance with the Canadian Privacy Act some supporting forms may have been removed from this thesis.

While these forms may be included in the document page count, their removal does not represent any loss of content from the thesis.
Conformément à la loi canadienne sur la protection de la vie privée, quelques formulaires secondaires ont été enlevés de cette thèse.

Bien que ces formulaires aient inclus dans la pagination, il n'y aura aucun contenu manquant.

\section{Canadä}




\begin{abstract}
Photonic crystals are periodic nanostructures whose dispersion relations can be regarded as the optical equivalent of electronic bandstructures from solid-state physics. Photonic quasicrystals are the rotational analogy of photonic crystals. These structures give rise to rotational extended states similar to the translational extended states, known as Bloch states, in photonic crystals. These rotational extended states appear as defect states because they are localized to certain sites within the photonic quasicrystal pattern. However, the pattern does not have physical defects to yield these states as in the case with photonic crystals. This thesis puts forward the case that these rotational extended states arise from crystal rotational symmetry. The rotational order (or foldness) of this photonic quasicrystal can be identified with an orbital angular momentum quantum number, $l$, which leads to $2 l+1$ rotational extended states. These states are called crystal angular momentum states and are similar to central potential states in atomic systems. The analogy is strengthened further by the discovery of Lamb shift states similar to those in atomic systems. Group theory predicts less than $2 l+1$ localized states but it is the unconventional scatterer shapes of the photonic quasicrystal that manifests the $2 l+1$ modes. Theoretical, simulated and experimental evidence is presented in this thesis for the existence of crystal angular momenturn states in photonic quasicrystals.
\end{abstract}




\section{Acknowledgements}

There is no doubt that this degree is dedicated to my dear parents. My father, Walid Mnaymneh, came here from Beirut, Lebanon to give his children opportunities that were not available in a country that, unfortunately, was being ravaged by war. He sacrificed his dreams so that his children can attain theirs. My mother, Khadije Batal Mnaymneh, was my best supporter. Her prayers, her sacrifices, her unconditional love and her friendship will always be in my heart. I love you two so much. As I am writing this, I am brought to tears thinking of the love and support my parents gave me and my siblings. My dear sister, Danya Mnaymneh and my brothers, Bassam and Marwan Mnaymneh, I know you guys were always in my corner and I am always in yours. To my children, Abdurahman and Noorah (and whoever comes after, you too!), you are my inspiration. When I look at you, you give me the resolve I need to press forward with any problem. I love you so much. To everyone else in my family, the Mnaymnehs and the Batals, especially Nizar, Munzer and Zaina Batal, thank you all so much for your prayers and support. To my dear father and mother-in-law, William Alexander McCrum and Maria Carmela Pinedo McCrum, thank you both so much for your solid support and invaluable advice. I have always listened to both of you with my heart. Thank you. Also my sisters-in-law, Vivian and Anna McCrum.

To all my brothers, especially Mohammad Agal, Hussein Alfiekh and Walaa Kholagi for your advice, guidance and classes. Thank you all so much for your prayers and support.

To my supervisor and friend, Professor Robert Claude Gauthier, thank you so much for your guidance in this endeavor, your patience and your support! To Professor 
Garry Tarr, thank you so much for your support and words of encouragement. You really are an excellent teacher! To my examination committee, thank you so much, especially Professor Jacques Albert. I thank you for bringing the best out of me! I will always remember this defense in the best light. To the support staff in the Department of Electronics thank you so much, especially Blazenka Power and Peggy Piccolo for your encouragement. A special deep gratitude to Rob Vandusen and Carol Adams. Your guidance and patience with me is very much appreciated. Thank you. I would like to thank Edith Post for her help.

To my student colleagues, Faisal Saleh, Winnie Ye, Keith Sarault, Kristen Medri, Scott Newman and Suthaharan Kanagaratnam thank you for all the interesting conversions we have had.

Chris, you get your own paragraph. I am sitting here trying to think of something witty to say. I realized that we have such a database of wit; it would be unfair to all the other statements if I only choose one. Seriously, Chris, in the little time I have known you, you have enriched my life more than you know my friend. Our discussions, our analysis...Chris...thank you so much.

Finally, last and absolutely not least, my best friend in this entire Dunya, my Sweet wife, Martha Elizabeth McCrum. Martha, I love you so much. There are no words to express how I feel about you except that I want to hold your hand and walk into Jannah with you. Thank you so much Sweetheart. Thank you for your patience, thank you for your support, thank you for your encouragement, thank you for our Children. Thank you, thank you, thank you. I love you so much. 


\section{Contents}

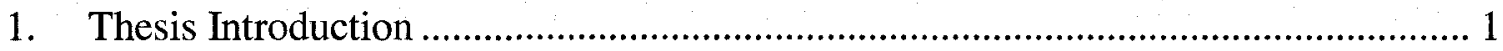

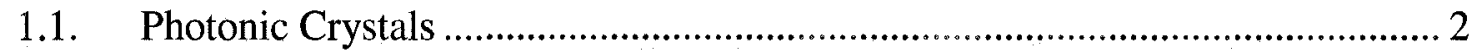

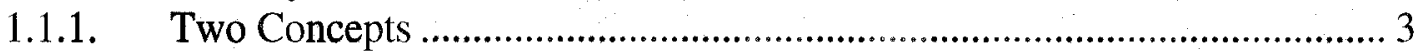

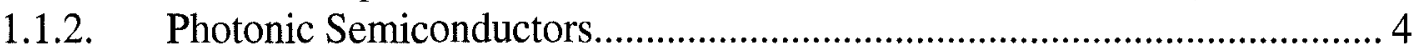

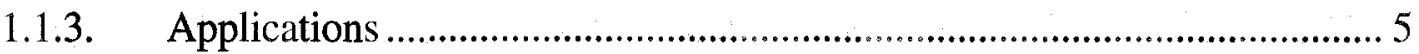

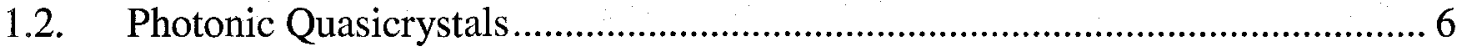

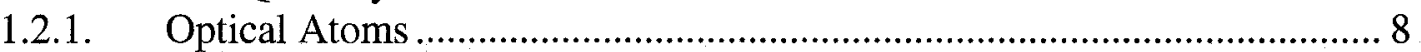

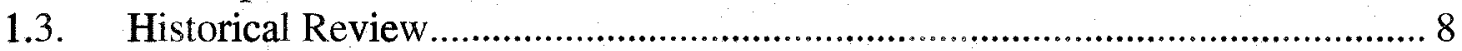

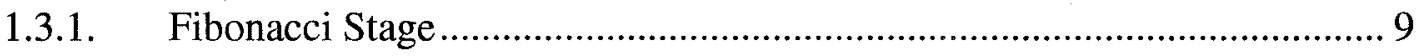

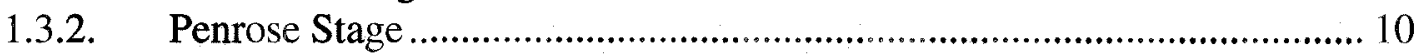

1.3.3. Thesis Context ................................................................................... 14

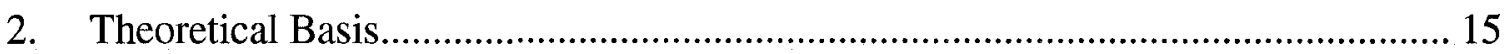

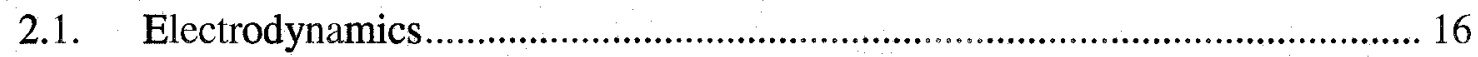

2.1.1. The Maxwell Equations ..................................................................... 16

2.1.2. Electrodynamics in a General Medium.................................................... 16

2.1.3. Operator Form of Maxwell's Equations .............................................. 18

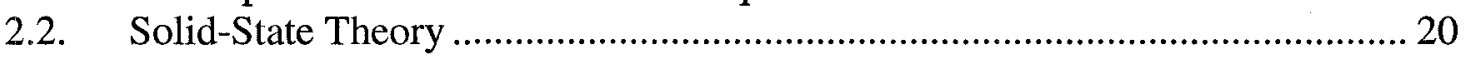

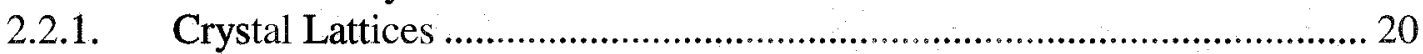

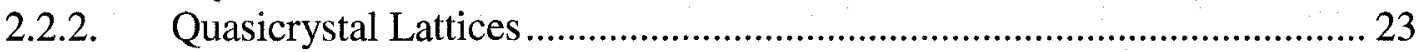

2.2.3. Form Factors ..................................................................................... 26

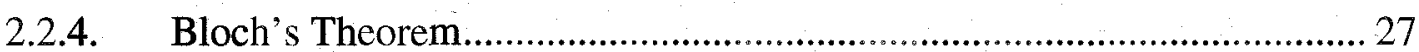

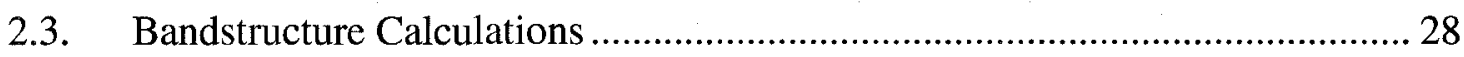

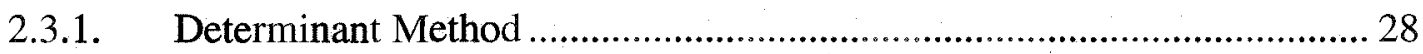

2.3.2. Planewave Analysis: Diagonalization Method......................................... 30

2.3.3. Planewave Analysis: Variational Problem.............................................. 32

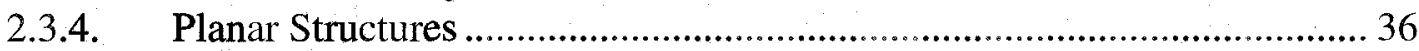

2.3.5. Green's Function Approach.................................................................... 46

2.3.6. The Need for a Numerical Approach....................................................50

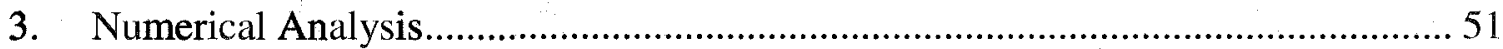

3.1. Photonic Quasicrystals Pattern Generation.................................................53

3.1.1. Dual Beam Holographic Lithography Technique ....................................53

3.1.2. Crystal and Quasicrystal Lattice Generation ............................................. 55

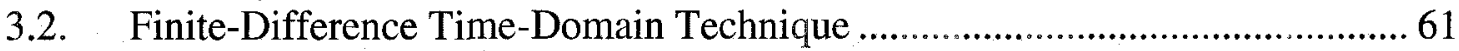

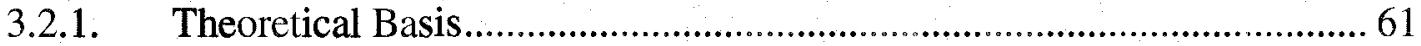

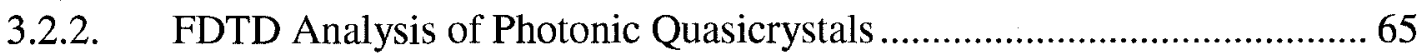

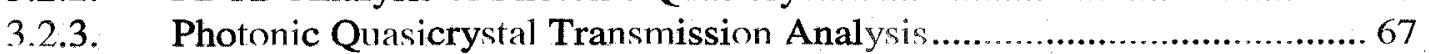

3.2.4. Photonic Quasicrystals Waveguides ......................................................... 72

3.2.5. Towards a Photonic Quasicrystal Device ……….................................... 77

3.2.6. Moveable Computational Domain Window ……..................................... 78

3.2.7. Photonic Quasicrystal Defect State Analysis.............................................. 82

3.2.8. Crystal Angular Momentum (CAM) Postulate........................................... 87

3.3. Finite-Element Method (FEM) Technique ...................................................... 90

3.3.1. General Theoretical Basis ....................................................................... 90 
3.3.2. Modeling PhC Bandstructures as Verification .......................................... 96

3.3.3. Importing PhQ Patterns for FEM Anälysis ............................................... 100

3.3.4. FEM Eigenvalue Analysis of PhQs ...................................................... 103

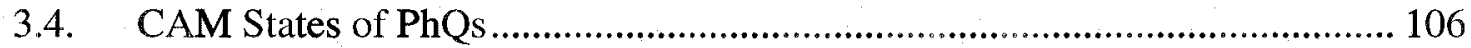

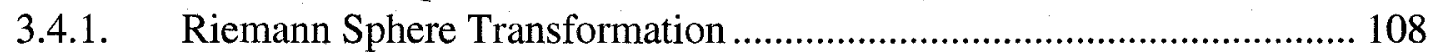

3.4.2. Group Theory Predications ................................................................ 110

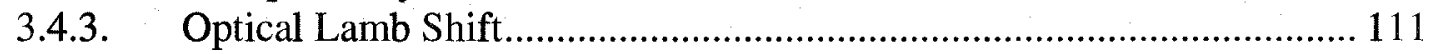

3.4.4. CAM States of Different PhQ Foldness.................................................. 112

3.5. Accessing Symmetries: Coupling Simulations ................................................ 113

3.5.1. Designing Three Systems of Observation.............................................. 113

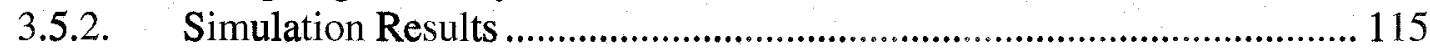

4. Fabrication: Accessing Symmetries................................................................. 118

4.1. Electron Beam Lithography (EBL) ............................................................. 119

4.1.1. Scanning Electron Microscopy For Writing …………………................ 119

4.1.2. Electron beam resist.............................................................................. 123

4.2. Image Processing for Electron-Beam Writing ................................................ 124

4.2.1. Pattern Preparation..................................................................................... 124

4.2.2. Nanometer Pattern Generation Software (NPGS) .................................... 127

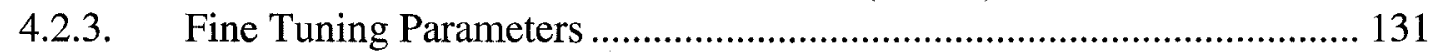

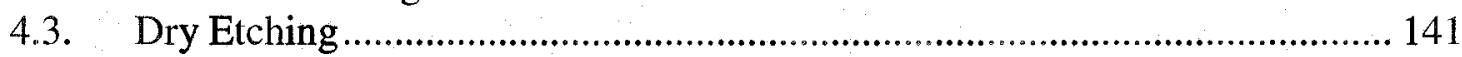

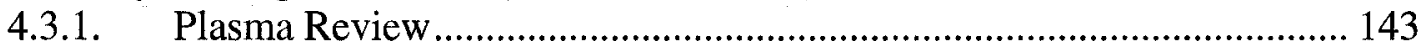

4.3.2. Electron-Cyclotron Resonance Etching ................................................ 144

4.3.3. Dry-Etching of Structures .................................................................. 145

4.4. Accessing PhQ Patterns for Measurement..................................................... 150

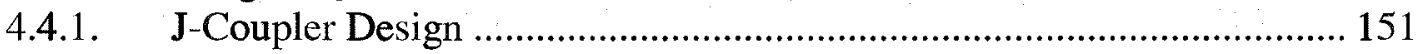

4.4.2. Waveguide Aligning …………............................................................ 157

4.4.3. Fabrication of Three Systems of Observation ........................................ 160

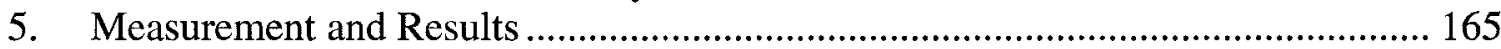

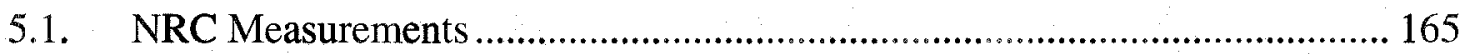

5.1.1. Measurement Setup and Equipment ....................................................... 166

5.1.2. Measurement Process................................................................................ 167

5.1.3. Measurement Result: System One ........................................................ 169

5.1.4. Measurement Result: System Two .......................................................... 176

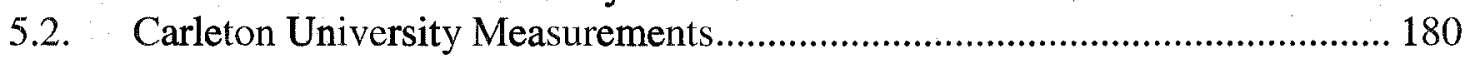

5.2.1. Measurement Setup and Equipment ..................................................... 180

5.2.2. Measurement Process............................................................................... 182

5.2.3. Measurement Results: System Three.................................................. 185

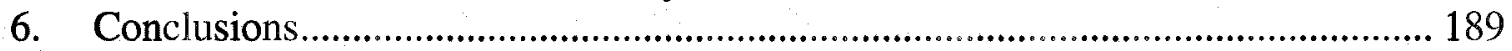

6.1. Summary of Results .................................................................................. 189

6.2. Summary of Thesis .................................................................................... 189

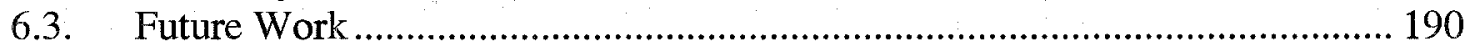

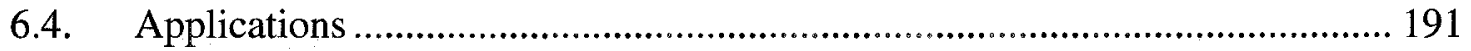

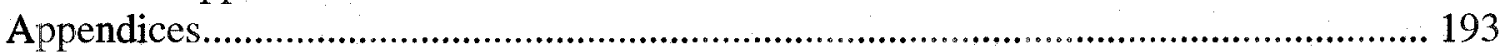

Appendix I: Algebraic Vector Spaces............................................................. 193

Algebraic Description ............................................................................................... 193

Hermitian Operators............................................................................................ 195 


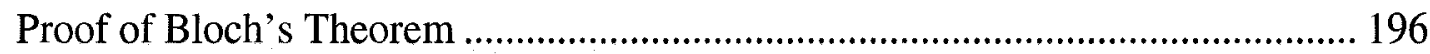

Appendix II: Green's Functions.......................................................................... 199

Density of States from Green's Functions .............................................................. 202

Appendix III: Diffusion Picture versus Wave Picture ..............................................206

Appendix IV: Noether's theorem............................................................................. 209

Appendix V: Gauge Invariance and the Electromagnetic Field .................................... 211

Appendix VI: Invariance of Schrödinger's Equation .............................................. 214

Appendix VII: SEM Architecture ....................................................................... 216

Appendix VIII: Applying Resist and Sample Preparation............................................ 223 


\section{List of Figures}

Figure 1.1. Example of photonic crystal structures. (a) One-dimensional (Bragg grating), (b) Two-dimensional, and (c) Three- dimensional. Dark and light regions are of different dielectric constants.

Figure 1.2. Defect engineering applications. (a) and (b) show line defects acting as optical waveguides and (c) shows physical point defects in PhCs actions as add/drop ports for specific wavelengths [6]

Figure 1.3. Creating PhQ structure from Penrose tilings. (a) Penrose tiling, (b) placing rods on all vertices and (c) the finished PhQ pattern.

Figure 2.1. (a) primitive cells of arbitrary shape in 2D crystals and (b) Wigner-Seitz

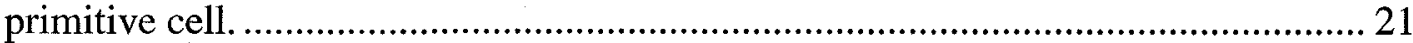

Figure 2.2. Primitive cells can be rotated through a special angle to construct an entire crystal. (a) Triangular cell, (b) square cell and (c) hexagonal cell.

Figure 2.3. Forbidden symmetries lead to an inability to tile all of space...................... 24

Figure 2.4. Penrose tiling................................................................................. 24

Figure 2.5. Scanning electron microscope image of quasicrystal 3D lattice of $\mathrm{AlCuFe}$ alloy. Dotted pentagon is drawn to highlight physical pentagon........................... 25

Figure 2.6. Dispersion relation for 1D periodic structure. Dashed line is the dispersion

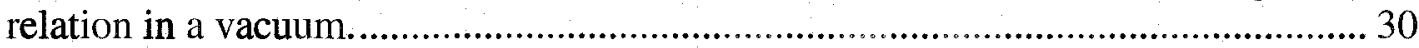

Figure 2.7. Unit cell of diamond lattice structure.................................................... 34

Figure 2.8. Dispersion curve obtained via matrix diagonalization. Shaded region

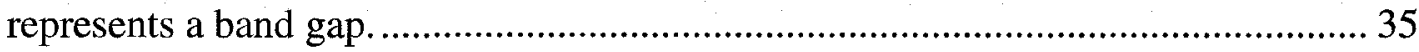

Figure 2.9. Brillouin zone for FCC structure......................................................... 35

Figure 2.10. Two-dimensional crystal. Square lattice with circular basis..................... 36

Figure 2.11. Photonic crystal and photonic quasicrystal. .......................................... 37

Figure 2.12. Octagonal PhQ using circular rods [8] ................................................ 37

Figure 2.13. Planewave diagonalization results for bandstructure of (a) square array of high dielectric rods in low dielectric background and (b) hexagon array of low dielectric holes in a high dielectric background.

Figure 2.14. Primitive cells for planewave analysis. (a) hexagonal array primitive cell

(b) 12-fold quasicrystal 'primitive' cell

42

Figure 2.15. Example of PhQ bandstructure calculated from the planewave expansion. 44

Figure 2.16. Top row images are of real structures and bottom row images are diffraction patterns. (a) Naturally occurring AlCuFe quasicrystal, (b) 10-fold $\mathrm{PhQ}$ fabricated in silicon, (c) 12-fold PhQs fabricated in silicon and (d) hexagon $\mathrm{PhC}$ fabricated in

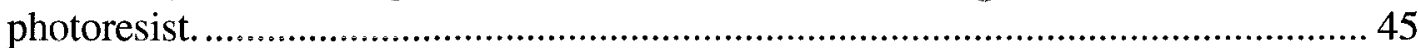

Figure 2.17. Obtaining the diffraction pattern on $\mathrm{PhQs}$ and $\mathrm{PhC}$ patterns.................... 45

Figure 2.18. Ten-fold symmetric Penrose tiling. High dielectric rods placed at vertices

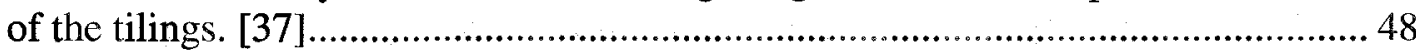

Figure 2.19. LDOS for PhQ structure in figure 2.18 (solid line) and square lattice of

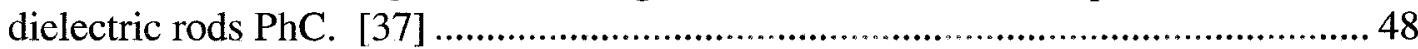

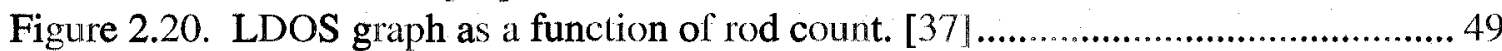

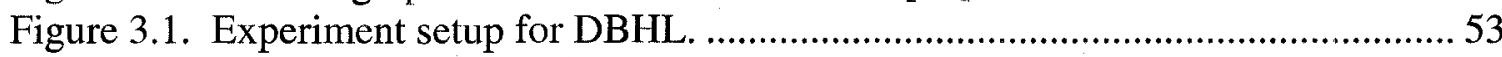


Figure 3.2. Planewave generation via the DBHL process.

Figure 3.3. Relationship between Euler angles of stage and plane equation coefficients.

Figure 3.4.. Interfering plane families (1) lead to crystal structures (2) with different lattice types (3). (a) Square lattice and (b) triangular lattice. Associated direction axes shown at far right.

Figure 3.5. Planewave schematic view of creating periodic structures and their relative threshold values in resist.

Figure 3.6. Physical structures of dual beam holographic exposure method. Bar length in both images is one micron. 58

Figure 3.7. Creating quasicrystal structures. (a) planewave schematic and (b) some set threshold ratio to generate physical structure.

Figure 3.8. PhQ patterns at different exposure thresholds yielding the different fill factors. Starting from top left, reading across, then down by rows, the thresholds of $7,8,10,12,14,15,16,18$ and 20 , out of a maximum of 24 .

Figure 3.9. Screen capture of Mathematica software generating quasicrystal patterns... 61

Figure 3.10. Finite-difference scheme and field definitions for the discrete 2D calculation lattice [48].

Figure 3.11. Stepping scheme for field computations. The $k$ term represents both $i$ and $j$ in equations 3.5 to 3.7 .

Figure 3.12. PhQ structure on a discretization grid. Black and white contrasts are related to dielectric contrasts. The solid line across shows location of the planewave source. Circles indicate observation points used for Fourier transform analysis.

Figure 3.13. TE polarization ( $\mathrm{Hz}$ component) transmission spectrum for a $10 \mu \mathrm{m}$ length 12 -fold $\mathrm{PhQ}$ of $50 \%$ fill factor. A band gap is present between the wavelengths of $1.05 \mu \mathrm{m}$ and $1.28 \mu \mathrm{m}$ and contains two defect states within.

Figure 3.14. TM polarization (Ez component) transmission spectrum for a $10 \mu \mathrm{m}$ length 12 -fold quasi-crystal of 50\% fill factor. Several band gap regions are present over the wavelength range displayed. Due to the rich nature of the band gaps for the TM polarization, one needs to explore in detail the optical properties of the 12-fold quasi-crystal for this polarization state of light. 70

Figure 3.15. Line plot of the transmission spectrum for the Ez component of the TM polarization plotted versus wavelength and dielectric fill factor. The larger of the band gaps is located in the flat zone about the $1.5 \mu \mathrm{m}$ wavelength value. A second large band gap is located in the $1.0 \mu \mathrm{m}$ wavelength range.

Figure 3.16. Line plot of the transmission spectrum for the Ez component of the TM polarization plotted versus wavelength and $0^{\circ}$ to $15^{\circ}$ propagation angle in $2.5^{\circ}$ increments. Through rotational symmetry, other propagation angles outside $15^{\circ}$ range can be rotated into the $0^{\circ}-15^{\circ}$ range displayed. The PhQ displays a uniform transmission spectrum over a propagation angle.

Figure 3.17. Dielectric contrast vs. band gap center wavelength. Dark region shows the relative size of the band gap.

Figure 3.18. Plot of the Poynting vector magnitude vs. wavelength and Fourier transform point location in the center of a $1.50 \mu \mathrm{m}$ wide waveguide centered on the PhQ. The top trace indicates that the low dielectric waveguide transmits strongly in the band 
gap range of the quasi-crystal and leaks light in those wavelength ranges which correspond to the transmission bands of the PhQ.

Figure 3.19. Transmission profile of the $1.5 \mu \mathrm{m}$ wavelength through a $1.50 \mu \mathrm{m}$ low index waveguide centered on the PhQ. The dimensions of the PhQ is $6 \mu \mathrm{m}$ across and 17

$\mu \mathrm{m}$ long. High transmission is observed for this wavelength................................... 76

Figure 3.20. High dielectric waveguide through 12-fold 20\% fill factor PhQ................ 76

Figure 3.21. Process towards PhQ DWDM Device. (a) Figure 3.15 indicates that different fill factor PhQs have different transmission characteristics. Line defects about a common wavelength can then be placed in each PhQ and different point defects with different resonance wavelengths can be designed. (b) Take a portion from each PhQ fill factor pattern and stitch pattern via the common line defect. (c) Continuous stitching or grading of (a) and (b) process to gain finer wavelength, $\lambda_{n}$, add/drop control

Figure 3.22. Movable Computational Window Domain (MCWD): (a) The shaded region in the PhQ pattern is the MCDW. (b) Arrows indicate that the MCWD can be scaled in size and (c) Arrows indicate that it can be moved around within the patttern. All FDTD features and analysis occur within this window only............... 79

Figure 3.23. Transmission spectrum for MCDW of width $10 \mu \mathrm{m}$ in 12-fold PhQ ........ 80 Figure 3.24. Transmission spectrum for MCDW of width $5 \mu \mathrm{m}$ in 12-fold PhQ. ............ 81

Figure 3.25. Transmission spectrum for MCDW of width $3 \mu \mathrm{m}$ in 12-fold PhQs........... 82 Figure 3.26. Sample size FDTD transmission runs of PhQ. Top trace shows large stop gap region of $10 \mu \mathrm{m} \mathrm{PhQ}$. Lower trace shows that 'defect' states have occurred because of sample size change to $3 \mu \mathrm{m}$. The solid circles show 'defect' states that were not present in the $10 \mu \mathrm{m}$ and the dashed circle shows that are perhaps in-band defect modes that have been made stronger because of sample size.......................... 83

Figure 3.27. FDTD domain for analysis of localized states. ............................................ 85

Figure 3.28. Fourier transform of observation points of FDTD defect state analysis inside

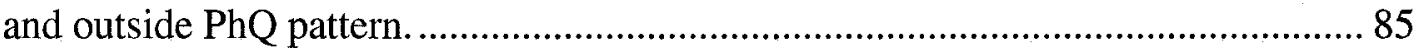

Figure 3.29. Defect mode profiles and where they occur in the transmission spectrum. (a) occurs in band gap at $1.56 \mathrm{um}$, (b) occurs in smaller band gap at $0.96 \mathrm{um}$, (c) occurs in band at $1.1 \mathrm{um}$, and (d) occurs in band at $1.24 \mathrm{um}$.............................................. 86 Figure 3.30. Results from [8]. (a) Eight-fold structure with gaps in DOS curve, (b) DOS curve showing frequency of (c) localized state profile. 88

Figure 3.31. Results from [9]. (a) Dodecagonal PhQ structure made from circular rods, (b) is the comparison between simulated and measured, where arrows point to localized modes and (c) is an example of localized modes found............................... 88

Figure 3.32. Basic finite elements. (a) One-dimensional. (b) Two-dimensional. (c) Threedimensional.

Figure 3.33. COMSOL screen captures are setting up primitive cells of (a) a hexagonal structure, (b) square structure and (c) vein structure. ................................................97

Figure 3.34. Lowest order field modes of eigenvalue problem of (a) a hexagonal structure, (b) square structure and (c) vein structure ...............................................97

Figure 3.35. Comparison between simulated results (right) and published results [] (left).

(a) hexagonal structure, (b) square structure and (c) vein structure.

Figure 3.36. Approximate bandstructure of PhQ using FEM technique. 
Figure 3.37. (a) Importing DXF image of PhQ, (b) shows the level of polygons required

to get curvature, and (c) is a close up of the polygons........................................... 102

Figure 3.38. Inversion of PhQ structure so that meshing will occur over a smaller area which will save computation cycles. (a) imported structure and (b) inverted structure.

Figure 3.39. Converting polygons into circles and ellipses............................................. 103

Figure 3.40. Finite element mesh of imported PhQ pattern........................................... 104

Figure 3.41. Example of eigenmodes found in FEM analysis. (a) appears to be a dipole mode and (b) appears to have 12 lobes but with half of the opposite polarity. ...... 104

Figure 3.42. Resemblance between eigenmodes and spherical harmonics. (a)

Eigenmodes, (b) top of sphere domain which harmonic functions are solved on, and

(c) isometric view of sphere domain. Color legend of 3.41 applies for (a). 105

Figure 3.43. Resemblance between eigenmodes and spherical harmonics of square array

$\mathrm{PhC}$ (top) and hexagonal array PhC (bottom). (a) Eigenmodes, (b) top of sphere domain which harmonic functions are solved on, and (c) isometric view of sphere domain. One can also view these as a mixture of 4-fold and 6-fold quasicrystals that have translational periodicity. Color legend of 3.41 applies for (a). 105

Figure 3.44. Localized optical modes in a 12-fold PhQ. The number of negative lobes (represented in red and a negative sign) or the number of positive lobes (represented in blue and a positive sign) equals the $m$ number. The wavelength at which these modes occurred are shown underneath each respective mode [10]

Figure 3.45. Stereographic projection of 2D PhQ pattern to 3D sphere. (a) Riemann sphere definition. (b) 2D PhQ pattern, (c) 2D to 3D transformation, (d) 3D projection of $2 \mathrm{D}$ sphere.

Figure 3.46. (a) PhQ CAM $m=1$ state, (b) Riemann sphere map of state, (c) equivalent spherical harmonic mode

Figure 3.47. Group theory predicated $m=0$ modes......................................................... 111

Figure 3.48. Rotational Bloch modes of octagonal (8-fold) PhQ................................. 112

Figure 3.49. Rotational Bloch modes for decagonal (10-fold) PhQs. .......................... 113

Figure 3.50. Three systems to simulate. (a) $10 \mu \mathrm{m}$ by $10 \mu \mathrm{m} 20 \%$ fill factor PhQ pattern with a silicon photonic wire of a width of $600 \mathrm{~nm}$ crossing the pattern at about 1.7 $\mu \mathrm{m}$ from the center. (b) $40 \mu \mathrm{m}$ by $20 \mu \mathrm{m} 20 \%$ fill factor PhQ pattern with a silicon photonic wire of a width of $600 \mathrm{~nm}$ crossing the pattern at about $1.7 \mu \mathrm{m}$ from the center. (c) $10 \mu \mathrm{m}$ by $10 \mu \mathrm{m} 20 \%$ fill factor PhQ pattern with a silicon photonic wire of a width of $600 \mathrm{~nm}$ crossing the pattern at the center but having a $3 \mu \mathrm{m}$ gap right at the center of the PhQ. 114

Figure 3.51. Placing photonic wire waveguides near sites shifts eigenvalues. (a) Mode with no waveguide nearby. (b) Mode with waveguide nearby, resulting in wavelength shift.

Figure 3.52. Larger PhQ pattern shows two types of localized modes. (a) Arrow points to center localized mode. (b) Arrow points to off-center localized mode. ............. 116

Figure 3.53. Simulation of third system. (a) $m=1$ excited and (b) $m=0$ excited. Note that they are Lamb shifted in terms of wavelength.

Figure 4.1. Carleton University's Scanning Electron Microscope (SEM) used for electron beam lithography. .............................................................................................. 119

Figure 4.2. PhQ generated from Mathematica. This is the $16 / 24$ or $20 \%$ fill factor... 124 
Figure 4.3. Image processing tool working on a PhQ structure ready for writing. ...... 125

Figure 4.4. CleWin editor showing converted PhQ image........................................ 126

Figure 4.5. Run file exposure parameter page......................................................... 128

Figure 4.6. Simulation of electron scattering in resist on a silicon substrate at (a) $10 \mathrm{kV}$ and (b) $20 \mathrm{kV}$ [52]

Figure 4.7. Optical gratings done in the first few days of conversion of Carleton University's SEM. Bar length is one micron.

Figure 4.8. First images of writing 2D PhCs. (a) One of the first exercises and (b) writing a "waveguide" along with the PhC. Bar lengths are ten microns............... 133

Figure 4.9. Stitching attempts by converted SEM. Bar lengths are ten micron............ 134

Figure 4.10. Evolution of SEM writing of 2D PhC structures. (a) SEM analysis of figure 4.8(b), (b) (c) and (d) show unexpected defects if writing parameters are not optimized - such as proximity effects, (e) better resist structures with intended defect structures, (f) is a close-up of one of the defects - and shows that the resist has developed consistently down to the substrate, (g) and (h) show 2D PhC and its close up, (i) shows a close up of the PhC holes to show curvature, (j) shows other defect structures, and (k) and (l) show a side profile of the resist walls. All bar lengths are one micron except ( $\mathrm{g}$ ) where the length is ten microns

Figure 4.11. PhQ SEM writing evolution. (a) Computer generated image of $12 / 24$ (50\% fill factor) $\mathrm{PhQ}$, (b) optical image of first time ever writing of $\mathrm{PhQ}$ at Carleton University's converted SEM, (c) SEM image of (b), (e), (f), (g) attempts of miniaturizing the $\mathrm{PhQ}$ so that its features are comparable with $1550 \mathrm{~nm}$ light, (h) perfected $\mathrm{PhQ}$ structure with the right exposure parameters. All bar lengths are one micron except (c) where the length is ten microns.

Figure 4.12. Defects can be added computationally and then written with the rest of the structure. (a) Shows a bent waveguide defect in the PhQ structure, while (b) shows a line and a point defect structure (point defect indicated by arrow). Bar length is one micron.

Figure 4.13. (a) A $10 \mu \mathrm{m}$ by $10 \mu \mathrm{m}$ PhQ structure and (b) a close up of the central region. The outer edges are badly developed while the central region is very close to being exactly like the computer-generated image. However, as (1) and (2) in (b) show, because of development, there is shrinkage and the posts are much smaller than they need to be. (1) has a diameter of $200 \mathrm{~nm}$, where it was supposed to be 250 $\mathrm{nm}$ and (2) has a length of $70 \mathrm{~nm}$ where it was supposed to be $120 \mathrm{~nm}$. These numbers are within range of the resolution capability of ZEP (which is $10 \mathrm{~nm}$ for 1 line). Bar length is one micron.

Figure 4.14. Compensation process: (a) choose a filter selection that enlarges white regions, (b) application of swelling feature, (c) transition to CIF for EBL, and (d) fabricated $\mathrm{PhQ}$ with the proper diameter middle rod size of $250 \mathrm{~nm}$. Bar length is one illicron.

Figure 4.15. PlasmaTherm( ${ }^{\circledR}$ SLR-772 Electron Cyclotron Resonance (ECR) Reactive Ion Etcher (RIE) System. Control module is on the left while the actual system in on the right. [Courtesy of Chris Raum]

Figure 4.16. Close up of control module for the ECR. [Courtesy of Kristen Medri] .... 142

Figure 4.17. Detailed description of ECR Method. (a) the top portion of the of the right hand side of figure 4.15. Item (1) is the microwave source and (2) depicts the tuning 
stubs. (b) is a schematic of the ECR system and the arrows from (a) to (b) connect the related regions in the physical picture and the schematic view.

Figure 4.18. Four etched PhQ structures of the same PhQ pattern. The substrate looks grainy. This is most likely grass residue. Bar length is one micron.

Figure 4.19. Higher density plasma smoothed background. The tilt in the image is an artifact of the SEM viewing system and is not physically there. Bar length is one micron

Figure 4.20. Going from unperturbed waveguide to perturbed waveguide to increase scattering. (a) Unperturbed waveguide-PhQ pattern. (b) Perturbed waveguide-PhQ pattern. Bar length is one micron.

Figure 4.21. Post analysis. (a) Tilted view of large area of PhQ. (b) Closer view. (c) Extreme close-up of post. All bar length are one micron except (c) where the length is $200 \mathrm{~nm}$.

Figure 4.22. Illustration of J-coupler geometry used for analysis and design............... 151

Figure 4.23. Slopes required to find y-components of outgoing rays............................. 153

Figure 4.24. J-Coupler design stages. (a) Base parabola halved and waveguide intersection points identified. (b) Waveguide region added. (c) Intersection function used to generate parabolic section of waveguide. (d) Taper region drawn. (e) Union function used to unite taper and parabolic region. (f) J-couplers overlaid with photomask design to insure J-couplers have appropriate dimensions. PhQ patterns would then be written between the J-coupler regions.

Figure 4.25. Physical fabrication of J-Coupler systems. (a) Optical image of J-coupler with photonic silicon wire. Size of pattern is $46 \mu \mathrm{m}$ by $26 \mu \mathrm{m}$. (b) SEM image of Jcoupler and $\mathrm{PhC}$ system, where bar length is one micron and (c) SEM images of Jcoupler and PhQ system, here bar length is $10 \mu \mathrm{m}$.

Figure 4.26. CIF Editor image of J-Coupler-PhC system with different layers shown. 157 Figure 4.27. Masking aligning with EBL pattern. (a) Unaligned EBL J-coupler-PhC pattern. This pattern is $26 \mu \mathrm{m}$ across and $46 \mu \mathrm{m}$ down. (b) Alignment mark written by electron-beam. (c) Unaligned photolithography waveguide mask. (d) Aligned EBL pattern to photolithography mask. [Courtesy of Kristen Medri]

Figure 4.28. Structures to be built to study CAM states on PhQs. (a) $10 \mu \mathrm{m} \times 10 \mu \mathrm{m}$ PhQ structure, (b) $40 \mu \mathrm{m}$ x $20 \mu \mathrm{m}$ PhQ structure, and J-coupler implementation with (c) $10 \mu \mathrm{m} \times 10 \mu \mathrm{m}$ PhQ structure 160

Figure 4.29. Preparation of PhQ pattern for integration with J-coupler system. (a) COMSOL® image of PhQ portion cropped from a J-coupler-PhQ system. (b) Photoshop@ image of cropped PhQ pattern with structure swelled to compensate for development and etching. (c) Larger swelling required for cropped pattern because swelling of (b) was not enough, yielding smaller rods.

Figure 4.30. PhQ structure with waveguide. No tapers will increase scattering so that the "defect" mode can be seen on the spectrum scan and detected by the IR camera. All bar lengths are one micron.

Figure 4.31. Fabricated larger PhQ system. (a) PhQ-waveguide system. Bar length is 10 microns. (b) Closer image of PhQ pattern, where bar length is one micron........... 163

Figure 4.32. J-coupler-PhQ system. (a) Large image showing the entire system. Arrows point to mismatches between EBL pattern and photolithography of waveguides. This will lead to scattering loss. The ellipse shows the EBL regions written in 
intentionally to keep the other waveguides in the photolithography mask from coming near the PhQ system. This will help reduce waveguide-waveguide coupling which would take energy away from the PhQ system. Bar length is 10 microns. (b) Close up of $\mathrm{PhQ}$ pattern. bar length is one micron.

Figure 5.1. General schematic for both systems used. The input stage consists of a tunable laser, and an input positioner 1. The sample sits on the positioner 2 and the output system begins at positioner 3. The output can then either be directed to a CCD camera and an OSA or computer system that models with output.

Figure 5.2. Measurement Setup at NRC. (a) Laser source, (b) tapered fiber input, (c) sample holder, (d) 40x objective output lens, (e) output filters, (f) optical detector, (g) IR output camera, (h) computer running LabVeiw® system. [Courtesy of Chris Raum]. 167

Figure 5.3. Waveguide outputs. (a) Optical output of $3.5 \mu \mathrm{m}$ width straight test waveguide and (b) $5 \mu \mathrm{m}$ width straight test waveguide.

Figure 5.4. Scan of straight waveguide vs. scan of waveguide containing PhQ (i.e. figure 4.30). (a) Straight waveguide scan. (b) Waveguide containing PhQ scan. (c) Large dip near simulated wavelength of defect mode wavelength of $1484 \mathrm{~nm}$. (d) Picture of the fabricated PhQ structure being tested.

Figure 5.5. Simulated $m=1$ CAM state with waveguide............................................ 170

Figure 5.6. Time-harmonic simulations of dip wavelength $1484 \mathrm{~nm}$. (a) the electric field,

(b) the power and (c) the simulated structure. 171

Figure 5.7. Power simulation results of dips at (a) $1495 \mathrm{~nm}$ and (b) $1517 \mathrm{~nm}$............. 172

Figure 5.8. Multiple measurements of localized mode wavelength. (a) Wavelength scan with input fiber changed slightly with respect to figure 5.3 measurement. (b) Wavelength scan with input fiber changed slightly with respect to (a) measurement. (c) Dip region at $1484 \mathrm{~nm}$ still prominent while at (d) coupling was reduced....... 173

Figure 5.9. Array of IR camera overhead pictures of PhQ. (a) Physical image. (b) and (c) Intensity pictures of the scattering at off-dip wavelength values. Arrows point to weak scattering in the area of the center. (d) Intensity picture of the localized mode shown exactly where the dip region occurs. The dash box represents the outline of the PhQ pattern.

Figure 5.10. Close up on other dip regions. (a) Figure 5.3. (b)Close up of other dip regions. (c), (d), and (e) Eigenvalue simulations of dip wavelengths.................... 175

Figure 5.11. Three scans of larger PhQ system. Curve (a), (b) and (c) all have consistent dip regions shown by 1,2 , and 3 . All curves show different coupling strengths. 176

Figure 5.12. System One and Two responses. (a) Straight waveguide response, (b) System 2 response and (c) System 1 response. Dips 1 and 3 coincides with both systems, confirming the existence of the CAM states at the center of the PhQ pattern. Dip 2 is in system 1 but not in system 2 . This is due to the weakness of this state. Dip 4 is in system 2 but not in system 1 because this is associated with a strong, off-center site that is not in system 1 . The X symbol indicates a dip on one curve and not in the other. 177

Figure 5.13. Three different strength eigenmode profiles of dip 3 wavelength of figure 5.10. Each eigenmode has slightly different eigenvalues, implying they are degenerate modes whose degeneracy is lifted by pattern structure. There/their/the strength would also depend upon coupling angles, etc. 
Figure 5.14. Simulated eigenmodes at (a) $1484 \mathrm{~nm}$ and (b) $1517 \mathrm{~nm}$ in the larger system.

Figure 5.15. Carleton University's Measurement Setup. (a) Agilent/HP 8168F tunable laser source, (b) Agilent/HP 86142A optical spectrum analyzer, (c) test jig, (d) monitor showing waveguide output mode and (e) microscope for viewing fibersample-objective lens alignment. [Courtesy of Chris Raum].

Figure 5.16. Close up picture of test jig. (a) Brass holder holds tapered fiber for input and output purposes, (b) stage that controls fine 3D motion and course 2D plane motion via knobs, (c) sample stage, (d) sample stage that controls 3D fine motion and course 1D motion (transverse to fiber), (e) 40x objective lens for output, (f) fiber stage which holds input tapered fiber, $(\mathrm{g})$ camera-fiber stage for switching between camera and input fiber, (h) camera-input fiber stage and 3D fine motion and 1D course motion control knobs, (i) output IR camera connected to monitor and objective lens, (j) overhead broadband light source, (k) free space coupler, (1) wavelength filter, (m) tapered fiber for input/output purposes and (n) input tapered fiber. [Courtesy of Chris Raum]

Figure 5.17. Positioning the Sample. (a) Overhead view of test jig and (b) close up of sample holder with sample (pointed to by arrow). [Courtesy of Chris Raum]...... 183

Figure 5.18. Result of careful adjustments. Arrow points to sharp images of waveguide mode of J-coupler-PhQ system. Inset is the third system to test, which is the Jcoupler-PhQ system. [Courtesy of Chris Raum]

Figure 5.19. Moving lens away and bring new input fiber in one roller stage. Arrow indicates direction of motion. [Courtesy of Chris Raum].....

Figure 5.20. First scans done at Carleton for a PhQ device. Curves (a) and (b) show dips in generally the same area in the spectrum which are indicated by the numbered arrows. However, the predicated wavelengths from Chapter 3 do not show dips. The reason is that the scan resolution is very coarse, which is a $2 \mu \mathrm{m}$ set resolution.

Figure 5.21. Post-measurement eigenvalue analysis of modes found in initial scan. (a) Dip 1 in both (a) and (b) scans, (b) dip 2 of (a) scan, (c) dip 3 of scan (a) and dip 4 of scan (b), modes (d) and (e) are at dips 2 and 3 of scan (b). These modes may be degenerate modes whose degeneracy is lifted by the DFF.

Figure 5.22. Two higher resolution scan of predication region. Dips 1 and 2 relatively correspond to the predicated CAM states that are Lamb shifted. The two scans overlapping in the right regions gives further evidence to the CAM state phenomena.

188

Figure 0.1. Correspondence between operator and its adjoint........................................ 195

Figure 0.2. SEM Column Description. (a) column housing electron beam source and electron optics system of magnetic lens, (b) sample chamber, (c) airlock port, (d) plunger that goes in airlock to deliver or retrieve sample in chamber, (e) variety of sample holders, (f) airlock control button, (g) airlock door control, (h) vertical control of chamber platform (i.e. bring sample closer or farther from columnchamber opening - also called the gun), (i) roll control of chamber platform,(j) yaw control of chamber platform and $(\mathrm{k})$ controls the xy motion of the chamber platform. 
Figure 0.3. Close up of control module of the SEM. (a) CRT where imaging and writing can be seen, (b) emitted beam current meter, (c) stage controller and joystick, (d) wobble control of stage, (e) magnification control, (f) focus control, (g) beam current control, (h) voltage and current main switches, (i) stigmators, (j) beam blanker, (k) gun direction control, (l) SEM to computer control switch and (m) the computer used to control the SEM for writing. 218

Figure 0.4. Physical and schematic drawing of electron beam column [65] .................. 220

Figure 0.5. Virtual source diameter. .......................................................................... 221

Figure 0.6. Resist spin station. (a) the spin area and spin chuck (not seen, inside), (b) control panel for spin station where spin rate and spin timing are controlled, (c) ZEP resist container, with eye droppers and petri dish near by, (d) hexamethyldisilazane (HMDS) which is applied on the substrate before any resist to promote adhesion, (e) hot plate set for low baking temperature - usually for photolithography resists and HMDS, and (f) higher temperature hotplate for ZEP. 224

Figure 0.7. Applying scratch on sample before loading into SEM. (a) sample and holder and (b) diamond tipped scribe. 225 


\section{Thesis Introduction}

In physics, the environment in which the dynamics of a system occurs is also a participant in the dynamics of that system. A clear example is the move from Newtonian physics to special relativity. In Newtonian physics, space and time was merely the stage in which dynamics occurred. In special relativity, space and time are active components that effect matter and field dynamics. Putting these concepts into a more practical sense, it was once thought the dynamics that obeyed conservation rules were intangible and only regulated by the laws of nature. However, if these dynamics occur within a crystal structure, the crystal itself then also has a part in these conservation rules, and the designer of the crystal can now manipulate these conservation laws. This is exactly how solid-state physics gave rise to present-day electronics.

In this thesis, the author will demonstrate the above concepts in optics by using a relatively new class of photonic band gap (PBG) materials, known as photonic quasicrystals (PhQs). In chapter 1, the author will introduce these PhQs in the context of photonic band gap materials and their history. In chapter 2, the author will describe the theoretical basis for analysis. The author will, in chapter 3, perform computer simulations on these structures and from the results, postulate the existence of new states in PhQs known as crystal angular momentum (CAM) states. In chapter 4, the author will discuss the fabrication of structures that will physically exhibit these CAM states, while chapter 5, the author will measure and physically obtain these results. The author will then conclude with chapter 6 and suggest future work in context of CAM states and PhQs. 
This chapter will first introduce PBG materials in context of structures known as photonic crystals (PhCs). It will discuss where PhCs come from, what is special about them, and their relevance in real world applications. Then, this chapter will introduce PhQs and discuss what they are, how they differ from PhCs, their relevance, and what makes them special. This introduction of PhQs will be followed by a historical review of the subject. This chapter will then conclude by putting this thesis into perspective with the field of PhQs.

\subsection{Photonic Crystals}

Photonic crystals are nanostructures having a spatially periodic modulation of the index of refraction that opens a forbidden gap of photonic energies in the electromagnetic dispersion relation known as a photonic band gap (PBG). This spatially periodic modulation of the dielectric can be in one, two, or three dimensions, as shown in figure 1.1.

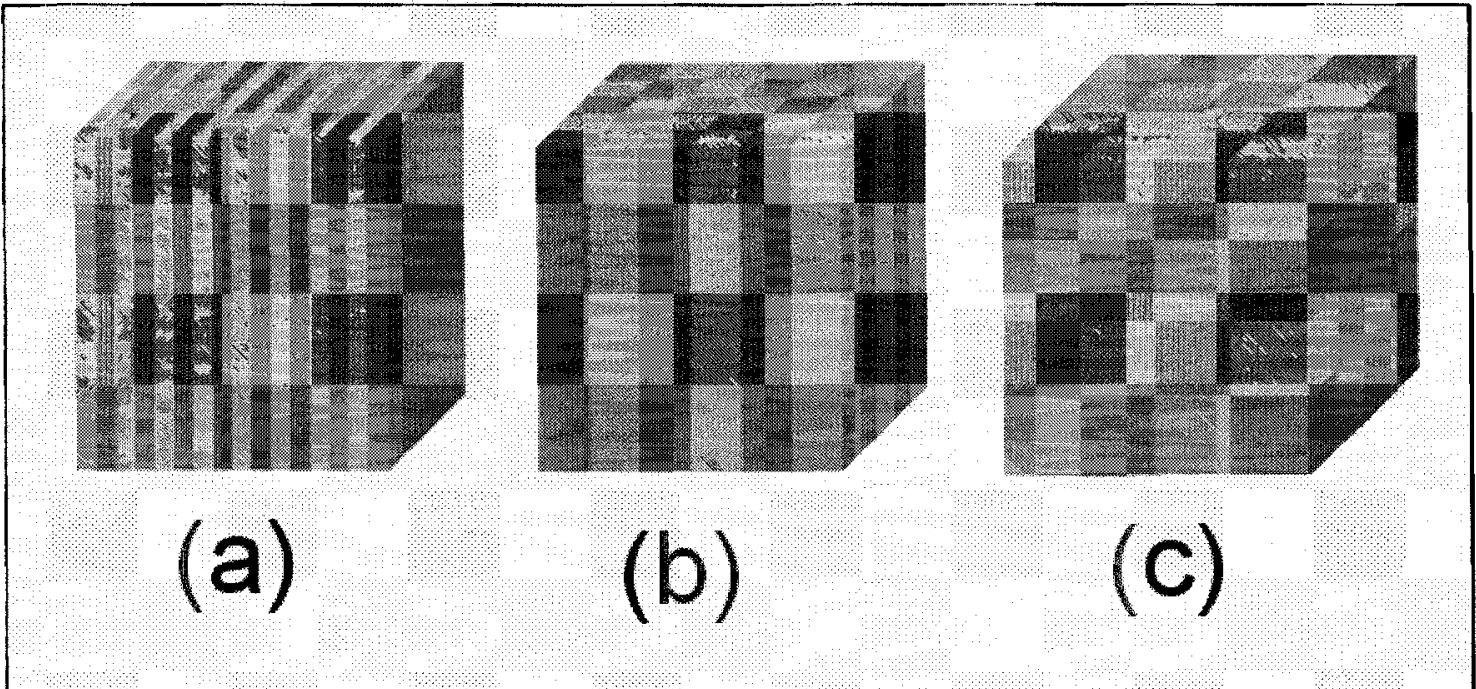

Figure 1.1. Example of photonic crystal structures. (a) One-dimensional (Bragg grating), (b) Two-dimensional, and (c) Three- dimensional. Dark and light regions are of different dielectric constants. 
Image (a) of figure 1.1 shows a 1D PhC. However, these structures have been around before the term 'photonic crystal' was coined. In 1887, Lord Rayleigh [1] made a significant discovery while studying the peculiar reflective properties of crystalline minerals. He discovered that these crystalline minerals had a narrow band gap prohibiting light propagation in a particular direction. This effect resembles a wavelength-dependent mirror and in 1971, Kogelnik et al. [2] utilized this property and suggested that a periodic structure in the gain medium of a laser's resonator cavity could replace the end mirrors. This backscattering effect (or feedback effect) improved the coherence of the laser light and reduced the overall size of the laser.

What makes PhCs different from 1D gratings is that one cannot physically take two or three 1D gratings and combine them to make 2D and 3D PBGs.

\subsubsection{Two Concepts}

In 1987, Eli Yablonovitch [3] and Sajeev John [4] independently published two papers addressing the area of inhibiting spontaneous emission and Anderson localization in electromagnetics, respectively, initiating the field of PhCs. Yablonovitch was trying to find a solution to control the spontaneous emission rate in different solid-state devices. For example, by surrounding a high-frequency switching transistor by a material that can inhibit spontaneous emission, one can reduce heating losses. Secondly, by surrounding a laser gain region by a material that can accelerate spontaneous emission, such as a cavity defect in a PBG, one can increase the laser gain. Yablonovitch had the idea to take 1D gratings and arrange them into $2 \mathrm{D}$ and $3 \mathrm{D}$ structures. However, this did not work due to the vector nature of electromagnetic field. In an indirect way, John understood what Yablonovitch failed to see. John was trying to demonstrate that localization could occur 
for photons in a similar way that localization occurs for electrons, as predicated by Anderson [5]. Showing localization for electrons is difficult because of the electronelectron interactions. Since there are no photon-photon ${ }^{*}$ interactions, John attempted to show that photon localization was possible, even though, as he acknowledged in the paper, this would prove to be difficult because of the vector nature of electromagnetic field. Ultimately he succeeded in giving the concept of PhCs a strong theoretical base.

\subsubsection{Photonic Semiconductors}

$\mathrm{PhCs}$ bring together two vast fields of study: electrodynamics and solid-state physics. Electrodynamics describes how matter influences the electromagnetic field to form dispersion relations. These dispersion relations can then be described in terms of solidstate concepts such as bandstructures and band gaps. If the matter is arranged like a crystal, that is, having an underlying lattice and a scatterer distribution attached to each lattice point (also known as a basis), the other solid-state concepts such as reciprocal lattices, Bragg planes, and Brillouin zones can be employed to describe how this dielectric crystal can control electromagnetic wave propagation. In a $2 \mathrm{D}$ crystal, the electromagnetic waves can be described by scalar wave equations, similar to the Schrödinger's electron wave equation in solid-state. Furthermore, the solid-state description becomes even more relevant when defects in the crystal structure are introduced to guide light or localize it for cavity purposes. Since one can control crystal lattice parameters and defect insertion points, the above concepts naturally lead to practical applications in photonic engineering.

\footnotetext{
* At least in first-order.
} 


\subsubsection{Applications}

The optical bandstructure of PhCs offers three types of engineering: (1) band-gap engineering, (2) band engineering and (3) defect engineering. The objective of band-gap engineering is to design PhCs with specified PBGs. These structures can be used as highly efficient mirrors in laser applications or optical insulators in planar integrated optics. The objective of band engineering is not so much the PBG but the shape of the bands. The slope of a band in the dispersion curve is the group velocity of the light in the $\mathrm{PhC}$. If the slope approaches zero, the group velocity also approaches zero. This feature can be used to increase laser efficiency, since the light would be traveling slower through the material and therefore excite more transitions. Defect engineering is concerned with introducing changes in dielectric contrast or material properties that break the local translational periodicity in some region of the PhC. This break in the local translational periodicity leads to localized states and any light introduced into these states have nowhere to go because of the surrounding PBG. These regions could be used as optical add/drop ports for wavelength multiplexers or if they involve a 1D defect such as a line, could then be used as waveguides. Figure 1.2 shows applications of the above descriptions. Images (a) and (b) show line defects and the images in (c) show point defects. 


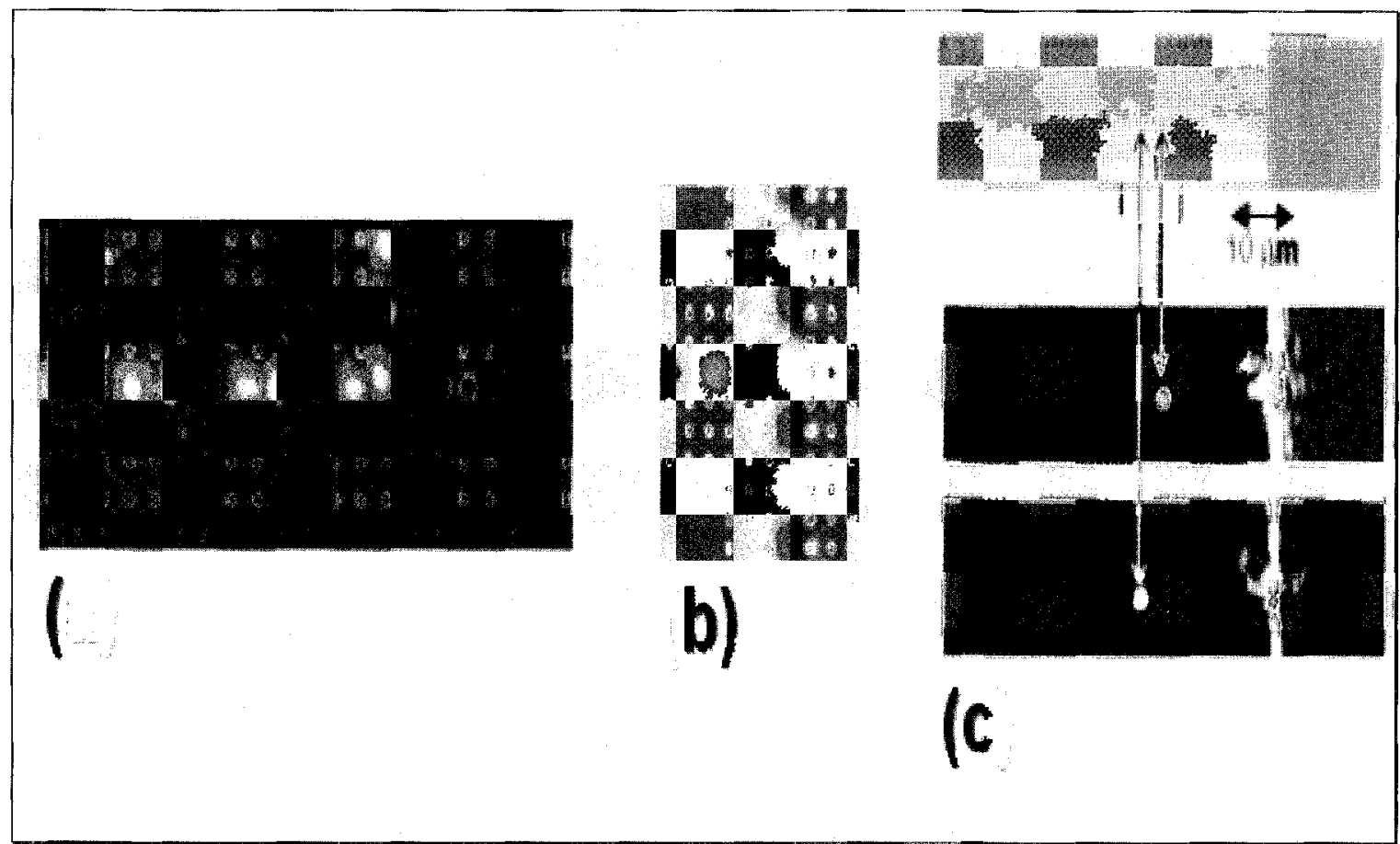

Figure 1.2. Defect engineering applications. (a) and (b) show line defects acting as optical waveguides and (c) shows physical point defects in PhCs actions as add/drop ports for specific wavelengths [6].

\subsection{Photonic Quasicrystals}

In the previous section, defect engineering was introduced as the study and exploitation of the local behavior of PhCs where the translational symmetry is broken. Extended modes of the translational symmetry, known as Bloch states, would become localized modes to these defects. What if the entire structure had no translational symmetry, yet had other types of symmetries or some type of order? These structures would not be amorphous, since amorphous structures have no distinguishing order or symmetries. These structures are called quasicrystals. Quasi $\cdots$, as defined in the Webster dictionary, means "resembling in some degree" and the definition of a crystal is that it "...has a regular repeating internal arrangement...". Putting these definitions together then 
describes a structure that has some type of order that resembles a crystal but is not exactly a crystal.

The author will, in chapter 2 of this thesis, go into further detail to define and describe photonic quasicrystals. However, it would be worthwhile to give a brief introduction about what quasicrystal structures look like.

Crystals are defined as having a regular repeating internal arrangement. This internal arrangement is referred to as a primitive cell. It must have a shape such that when tiled over all space, it must fill all space without there being a single gap. If this is the case, one has a crystal. If one requires a finite set of different shaped primitive cells to fill all space then one has a quasicrystal. For crystal primitive cells, the shapes are limited and can only have a 2, 3, 4 or 6-fold rotational symmetry. Primitive cells with other rotational symmetries than these will not be able to fill all space without any gaps. They require two or more other tilings to fill space. It was once believed these structures were only mathematical curiosities. However, in 1984, Daniel Shechtman [7] discovered an icosahedral phase of metallic alloys. This phase had long-range orientational order and no translational symmetry. This was the first time that such an aperiodic structure was discovered. This discovery turned quasicrystal structures from mathematical curiosities into scientific curiosities.

With this physical discovery established, researchers in the photonics community wondered if a quasicrystal analogy to PhCs would also produce bandstructures and band gaps. Such structüres would be called photonic quasicrystals (PhQs). In 1998, Chan et al. [8] discovered that 2D PhQs did indeed have in-plane bandstructures and band gaps just like 2D PhCs. However, he also found something unique about PhQs: they had localized 
states that did not have the same properties as the localized states that occurred in disordered media. He offered no explanation but took note that something was unique about them. This thesis is the first comprehensive explanation of this unique phenomenon.

\subsubsection{Optical Atoms}

The localized states found by Chan et al. [8] were in 8-fold rotational symmetric PhQs that did not have physical defect sites, although he did study such situations. In 2003, Wang et al. [9] found localized modes in defect-free 12-fold PhQs. He offered an explanation that involved a competition effect between self-similarity of the long-range order and non-periodicity or disorder. However, no one in the literature, with exception of Mnaymneh et al. [10], has put forth the idea that these localization modes in defectfree PhQs are actually Bloch mode equivalents in a rotational space. The rotational foldness of the PhQs can be related to an orbital angular momentum. This equivalence leads to various localized modes that resemble electronic states of a central potential. The sites that allow these localized modes can be viewed as optical atom sites with the localized modes of different sites interacting to make bands. Essentially, the PhQ is more like an optical material made up of optical atoms. This description is a unique one, and is also the basis of this thesis. However, it would be worth doing a historical review to put this present work into perspective.

\subsection{Historical Review}

The historical review of the PhQs is presented here in two stages: a Fibonacci stage and a Penrose stage. The Fibonacci stage actually precedes PhCs. The reason for this is that 
work in localization in disordered structures was becoming popular due to the 1977 Noble Prize of Philip W. Anderson in the area of electron localization. Researchers knew that periodic structures lead to extended wave states and disordered structures lead to localized wave states. The question at that time was what kind of states would occur in structures that were neither periodic nor disordered? The Fibonacci sequence was the ideal candidate to study because it was (a) neither periodic nor disordered and (b) it was one-dimensional. Penrose photonic structures were proposed after $\mathrm{PhCs}$ were discovered. Researchers wanted to determine if $2 \mathrm{D}$ structures that were neither periodic nor disordered had bandstructures with band gaps.

\subsubsection{Fibonacci Stage}

Briefly, a Fibonacci sequence is defined by the following recurrence relation,

$$
F(n)=\left\{\begin{array}{cc}
0 & \text { if } n=0 \\
1 & \text { if } n=1 \\
F(n-1)+F(n-2) & \text { if } n>1
\end{array}\right.
$$

Each number is the sum of the two preceding numbers. The first paper that gave a thorough analysis of aperiodic media that was stacked according to the Fibonacci sequence in the field of optics was a paper written in 1987 by Kohmoto et al. [11]. They mathematically analyzed a multilayer stack composed of two types of layers, each with different dielectric constants, that followed a Fibonacci sequence in layer thickness. They found that the spectrum of these structures possessed a fractal appearance. More specifically, their spectral response appeared to be that of a Cantor set. Diffraction experiments performed by Gellermann et al. [12] showed that when the stack become larger, the transmission dips also increased in number and in strength. They considered 
this to be evidence of photon localization since one of the signs of localization is that as the sample size becomes larger, the diffusion coefficient renormalizes to zero. Since that time, many papers in the literature have further investigated Fibonacci stacking sequences of different dielectric materials that yield very interesting spectrums $[13,14,15]$.

Penrose 2D lattices show fundamentally different spectra than the 1D aperiodic structures [16]. These 2D lattices do not present self-similarity and show degenerate states at the center of the spectrum which are separated from the rest of the states by a sizable gap [17]. It is these $2 \mathrm{D}$ aperiodic lattices that this thesis will deal with and provide a theory to describe their spectral response.

\subsubsection{The Penrose Stage}

It was Roger Penrose [18] who discovered that the minimum number of non-Bravais tilings required to cover all space was two. These tilings were first applied to research in photonics in 1998 by Chan et al. [8]. They constructed the PhQ pattern by placing rods at the vertices of the Penrose tiling pattern. Figure 1.3 shows a transition from Penrose tilings to a PhQ pattern with rods. Image (a) of figure 1.3 shows a Penrose tiling. Image (b) shows rods being placed at all vertices of the pattern and image (c) is the finished PhQ pattern. 


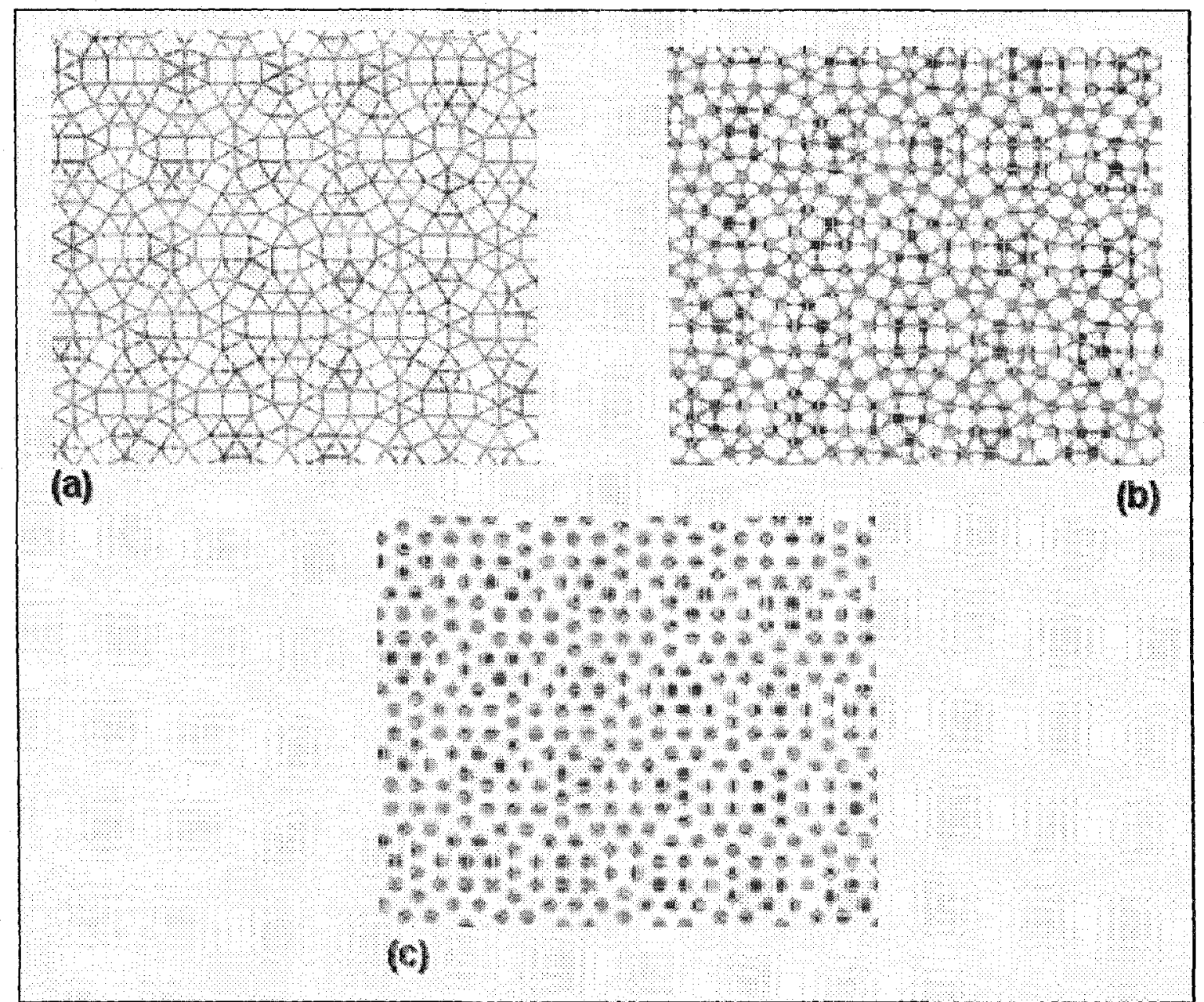

Figure 1.3. Creating PhQ structure from Penrose tilings. (a) Penrose tiling, (b) placing rods on all vertices and (c) the finished PhQ pattern.

After generating the patterns, Chan et al. [8] showed that band gaps were present in the spectral response of these patterns. They also investigated defects within these patterns. It was this first paper on $2 \mathrm{D}$ PhQs that noted the existence of localized states that had nothing to do with defect or disorder. Not much was discussed about this until the work of Wang et al. [9] in 2003. In between these two papers there was a lot of work in characterizing the bandstructure of PhQs. In 1999, two papers by Cheng et al. [19] and Jin et al. [20] studied line and point defect properties of PhQs and noted that their defects were more wavelength selective than PhCs. This makes them ideal candidates for dense wavelength division multiplexing (DWDM) systems. 
In 2000, Zoorob et al. [21,22] showed that the PhQ pattern allowed one to use a lower dielectric contrast to make PBGs. Also in this year, work on PhQ diffraction patterns and their theoretical link to planewaves [23,24] was published. Furthermore, the first paper to investigate short-range dependency of PBGs in PhQs [25] in the microwave region was also published this year. Short-range dependency means that number of lattice points with basis required to setup the PBG. This paper is important because it shows that the bandgap creation mechanism is different from the localization mechanism found in disordered structures.

In 2001, Kaliteevski et al. [26] wrote more on the diffraction results of PhQs and used these diffraction results to actually design $\mathrm{PhQs}$ in direct space from a Fourier transform [27]. Archimedean-like PhQs [28] were also presented in this year and showed similar results to previous PhQs. Also in this year, there was the first reference to using tight-binding theory for describing mode propagation via localized mode-hopping in a $\mathrm{PhQ}$ where defects were introduced to provide localization sites [29].

In 2002, another paper added further evidence towards short-range effects in PhQs and also noted that some transmission dips in PhQs depended upon the incident angle of the incoming light [30]. In 2003, the finite element method (FEM) was first applied to $\mathrm{PhQ}$ analysis showing excellent agreement with results found with the finite difference time domain (FDTD) technique [31]. The FEM technique lends itself easier to eigenvalue analysis so that more resolution of spectra can be done on PhQs. This year was also an important year for the discovery of localized modes in defect-free PhQs [9], which are localized modes generated from an inherent property of the quasicrystalline geometries rather than imperfections in the pattern geometry. 
In 2004, lasing applications utilizing PhQ defect modes [32] and defect-free defect modes PhQs [33] were reported. In addition to this, a technique known as dualbeam multiple-exposure holographic lithography developed by Gauthier and Mnaymneh was used to generate physical PhQs in silicon [34]. This technique could be used to mass-produce PhQ pattern templates. Also in this year, a new type of structure was proposed by Horiuchi et al. [35] known as a circular photonic crystal (CPC).

In 2005, J. Chaloupha et al. [36] expanded on the idea of Horiuchi et al. and further developed the theory for CPC. In this paper, the first reference to a rotational Bloch number is made, and allows the analogy between Bloch states in translational systems and rotational systems to be explored further, as is done in this thesis. In addition to this, A. Della Villa et al. [37] published an important paper on the topic of band formation in PhQs. They propose that there are two types of band formation mechanisms: a short-range and a long-range mechanism. Results from this paper are very important to the work in this thesis. In addition, more applications for DWDM systems using PhQs were also proposed in this year [38]. Furthermore, in 2005 Gauthier and Mnaymneh [39] presented an FDTD analysis of 2D PhQs that had unconventional scatterer shapes and dielectric fill factors. The result was that large band gap regions were found.

In 2006, further research on defect-free localized states in PhQs was published using an orbital theory [40]. This paper also utilizes group theory to establish the existence of these localized modes. Gauthier and Mnaymneh [41] discovered localized modes in unconventional scatter shape PhQs and studied their mode profiles using FDTD. Furthermore, A. Della Villa et al [42] published additional results on localized 
modes in defect-free Penrose lattices, and concluded that these modes should be considered as an inherent property of the quasicrystalline geometries. Subsequent papers on PhQ applications [43,44] were also presented in this year. In 2007, Gauthier [45] extended PhQs to PhQ fibers by studying the out-of-plane propagation of these localized modes.

\subsubsection{Thesis Context}

In 2007, Mnaymneh et al. [10] brought together all the findings of localized modes in defect-free PhQs into the theory of crystal angular momentum states. They demonstrated the existence of these crystal angular momentum states using FEM simulations and experiments in the near infrared regime (i.e. $\mathrm{S}$ and $\mathrm{C}$ channel ranges). Up until this time, all physical investigation on localized modes in PhQs had been done using large structures in the microwave regime.

From the previous section it can be appreciated that the area of PhQs is still new and quite active. It also shows that the theory governing these structures is still not fully developed. However, this thesis presents a strong argument that PhQs should be considered as optical atomic systems whose states are governed by the geometry and scatterer shape of the PhQ. Hence, the next chapter begins this thesis formally and provides the theoretical basis for the argument of crystal angular momentum in PhQs. 


\section{Theoretical Basis}

In this chapter, the author describes the theoretical tools required to study photonic quasicrystals. These tools come from two broad fields of study: electrodynamics and solid-state physics. While tools from these two fields can provide a complete theory for photonic crystals, photonic quasicrystals do not have such a rigorous analytical theory. This is because concepts within solid-state theory, such as Bragg planes and Brillouin zones, do not apply to PhQs. This means any simulation work for PhQs needs to be purely numerical. Regardless, it is still beneficial to use semi-analytical methods from solid-state theory to obtain a sense of how photonic quasicrystals interact with waves propagating within them. The author, in this chapter, will deal with this topic and discusses the application of two semi-analytical methods from solid-state physics: planewave analysis and Green's function methods.

The two most important results found in this chapter are: (a) while photonic quasicrystals have no translational symmetry but not disordered, they have a bandstructure with band gaps; and, (b) there are two types of PhQ band gaps: (i) those created from short-range interactions and (ii) those created from long-range interactions. All the above results allows PhQs to be used as optical components such as waveguides, laser, fibers, resonators, and add/drop ports. The second result shows that the bandstructure of $\mathrm{PhQs}$ is fundamentally different in origin from $\mathrm{PhCs}$ because the bandstructure of PhCs arises only from short-range interactions. 


\subsection{Electrodynamics}

\subsubsection{The Maxwell Equations}

The electrodynamics in vacuum is governed by the Maxwell's equations,

(Faraday's Law)

(Ampère-Maxwell's Law)

(Gauss' Law for Electrostatics)

(Gauss' Law for Magnetism)

$$
\nabla \times \mathbb{E}(\mathbf{r}, t)+\frac{\partial}{\partial t} \mathbb{B}(\mathbf{r}, t)=0
$$

where $\mathbf{E}(\mathbf{r}, t)$ is the electric field vector, $\mathbf{B}(\mathbf{r}, t)$ is the magnetic flux density vector, $\rho(\mathbf{r}, t)$ and $\mathbf{J}(\mathbf{r}, t)$ are the charge and current densities, respectively. The term $\varepsilon_{0}$ is the permittivity of free space. In order to describe the electrodynamics in PhCs and PhQs, one has to introduce the terms in Maxwell's equations that represents such structures.

\subsubsection{Electrodynamics in a General Medium}

The structure and material of the medium in which the electromagnetic field propagates is represented by the constitutive relations,

$$
\begin{gathered}
\mathbf{D}(\mathbf{r}, t)=\varepsilon(\mathbf{r}, \omega) \mathbf{E}(\mathbf{r}, t)=\varepsilon_{0} \varepsilon_{r}(\mathbf{r}, \omega) \mathbf{E}(\mathbf{r}, t) \\
\mathbf{B}(\mathbf{r}, t)=\mu(\mathbf{r}, \omega) \mathbf{H}(\mathbf{r}, t)=\mu_{0} \mu_{r}(\mathbf{r}, \omega) \mathbf{H}(\mathbf{r}, t)
\end{gathered}
$$

where $\mathbf{D}(\mathbf{r}, t)$ is the electric flux density, $\mathbf{H}(\mathbf{r}, t)$ is the magnetic field vector, $\mathcal{E}_{r}(\mathbf{r}, \omega)$ is the relative electric permittivity, $\mu_{r}(\mathbf{r}, \omega)=1$ is the relative magnetic permeability (which 
is set to 1 for reasons described below), and $\mu_{0}$ is the permeability of free space. The constitutive relations are the entry points in which the matter's structure and material effects control the electromagnetic waves. This is where solid-state physics enters into electrodynamics. Putting equations 2.5 and 2.6 into equations 2.1 to 2.4 , one gets,

$$
\begin{gathered}
\nabla \times \mathbf{E}(\mathbf{r}, t)+\frac{\partial}{\partial t} \mathbf{B}(\mathbf{r}, t)=0 \\
\nabla \times \mathbf{H}(\mathbf{r}, t)+\frac{\partial}{\partial t} \mathbf{D}(\mathbf{r}, t)=\mathfrak{J}(\mathbf{r}, t) \\
\nabla \cdot \mathbf{D}(\mathbf{r}, t)=\rho(\mathbf{r}, t) \\
\nabla \cdot \mathbf{B}(\mathbf{r}, t)=0 .
\end{gathered}
$$

The Maxwell equations are first-order coupled partial differential equations, and other than in very simple problems, such equations are hard to solve exactly. If a few assumptions about the medium are made, the equations can be decoupled and put into an operator form, which can then make use of matrix techniques for computation.

The first assumption is that the medium is sourceless and charge free,

$$
\rho(\mathbf{r}, t)=0 ; \mathbf{J}(\mathbf{r}, t)=0 .
$$

Secondly, the non-linear effects of the medium are so weak that one cannot observe them without great distances of propagation or enormous field strengths. Thirdly, the medium is non-magnetic so $\mu_{r}(\mathbf{r}, \omega)=1$. Fourthly, $\varepsilon_{r}(\mathbf{r}, \omega)$ is real which means the medium is lossless and finally, the wavelength range of the radiation will allow $\varepsilon_{r}(\mathbf{r}, \omega)$ to be relatively frequency independent: $\varepsilon_{r}(\mathbf{r}, \omega) \rightarrow \varepsilon_{r}(\mathbf{r})$. Applying these approximations to equations 2.7 to 2.10 one gets, 


$$
\begin{gathered}
\nabla \times \mathbf{E}(\mathbf{r}, t)+\mu_{0} \frac{\partial}{\partial t} \mathbf{H}(\mathbf{r}, t)=0 \\
\nabla \times \mathbf{H}(\mathbf{r}, t)+\mu_{0} \varepsilon_{r}(\mathbf{r}) \frac{\partial}{\partial t} \mathbf{E}(\mathbf{r}, t)=0 \\
\nabla \cdot\left\{\mathcal{E}_{r}(\mathbf{r}) \mathbf{E}(\mathbf{r}, t)\right\}=0 \\
\nabla \cdot \mathbf{H}(\mathbf{r}, t)=0 .
\end{gathered}
$$

Equations 2.12 to 2.15 are the starting relations when analyzing sourceless electromagnetic propagation in inhomogeneous structures such as PhCs and PhQs.

\subsubsection{Operator Form of Maxwell's Equations}

Due to the assumption of a sourceless environment, it is easy to decouple equations ${ }^{*} 2.12$ and 2.13. The uncoupled equations are then,

$$
\begin{gathered}
\frac{1}{\varepsilon_{r}(\mathbf{r})} \nabla \times\{\nabla \times \mathbf{E}(\mathbf{r}, t)\}+\frac{1}{c^{2}} \frac{\partial^{2}}{\partial t^{2}} \mathbf{E}(\mathbf{r}, t)=0 \\
\nabla \times\left\{\frac{1}{\varepsilon_{r}(\mathbf{r})} \nabla \times \mathbf{H}(\mathbf{r}, t)\right\}-\frac{1}{c^{2}} \frac{\partial^{2}}{\partial t^{2}} \mathbf{H}(\mathbf{r}, t)=0 .
\end{gathered}
$$

Seeking all time-harmonic solutions of equations 2.16 and 2.17 of the form,

$$
\begin{aligned}
& \mathbf{E}(\mathbf{r}, t)=\mathbf{E}(\mathbf{r}) e^{-i \omega t} \\
& \mathbf{H}(\mathbf{r}, t)=\mathbf{H}(\mathbf{r}) e^{-i \omega t}
\end{aligned}
$$

where $\omega$ is the angular frequency of the modes. Applying 2.18 and 2.19 to equations 2.16 and 2.17 , one can then write the Maxwell's equations as operator equations,

\footnotetext{
* Otherwise, one would have to represent the fields in terms of gauge invariant potentials (refer to Appendix VI).
} 


$$
\begin{aligned}
& L_{E} \mathbf{E}(\mathbf{r})=\frac{1}{\varepsilon_{r}(\mathbf{r})} \nabla \times\{\nabla \times \mathbf{E}(\mathbf{r})\}=\frac{\omega^{2}}{c^{2}} \mathbb{E}(\mathbf{r}) \\
& L_{H} \mathbf{H}(\mathbf{r}) \equiv \nabla \times\left\{\frac{1}{\varepsilon_{r}(\mathbf{r})} \nabla \times \mathbf{H}(\mathbf{r})\right\}=\frac{\omega^{2}}{c^{2}} \mathbf{H}(\mathbf{r})
\end{aligned}
$$

where $L_{E}$ and $L_{H}$ are differential operators defined by the first equality. By defining these operators, the mathematics of linear transformations and eigenvalue problems are now accessible for characterizing the electrodynamics within the medium defined above. These methods lend themselves to matrix methods that are used to perform simulations on a computer.

Operators can be represented as matrices, and field quantities are represented as column and/or row vectors. If the field quantities in equations 2.20 and 2.21 are not zero, then these two equations are eigenvalue equations. Eigenvalue equations are common equations in mathematical physics. If the operators are Hermitian, then this mathematically guarantees that the eigenvalues are always real, and that the eigenvectors associated with different eigenvalues are always orthogonal. This means that the eigenvectors of these Hermitian operators are complete since there is no larger space [49]. This important feature allows one to find the relation between a wave's frequency and its wavevector in some medium. This relation is called the dispersion relation in electrodynamics, and in solid-state physics it is known as the bandstructure.

Finding the dispersion relation for PhCs requires some understanding of crystal lattices. This is where solid-state physics terminology becomes important. Finding the $\mathrm{PhQ}$ dispersion relation will then be discussed in light of the dispersion relations of PhCs. 
This highlights the concept of moving from what is known (i.e. PhCs) to what is unknown (i.e. PhQs) via analogies.

\subsection{Solid-State Theory}

\subsubsection{Crystal Lattices}

Solid-state physics is primarily concerned with crystalline solids. Crystals can be defined as a set of translationally symmetric lattice points with some distribution of wave scatterers at each lattice point. A portion of the crystal that can be tiled over all space to reconstruct the entire crystal is called a unit cell. This unit cell can be mathematically represented as [46],

$$
\mathbf{R}=n_{i} \mathbf{a}_{i}
$$

where $n_{i}$ are integers, $\mathbf{a}_{i}$ is a basis vector of the unit cell. Einstein summation is assumed. In this work, $i$ will only go as high as three (i.e. the highest spatial dimension dealt with will be three dimensions).

If the unit cell contains precisely one lattice point, it is called a primitive cell. If the lines or planes of the primitive cell are perpendicular bisectors of lines joining nearest neighbor lattice points, the primitive cell is known as a Wigner-Seitz cell. Figure 2.1 shows primitive cells of arbitrary shape (a) and the Wigner-Seitz cell (b). Notice that one can tile all space with the shape and its rotations through nultiples of a special angle. Doing this, one constructs the entire crystal, as shown in figure 2.2.

Due to the translational symmetry of the crystal lattice, these lattice points can be collected into sheets (in 3D) or lines (in 2D) and represented as families of planewaves. The collection of wavevectors of these planewave families is called the reciprocal lattice. 


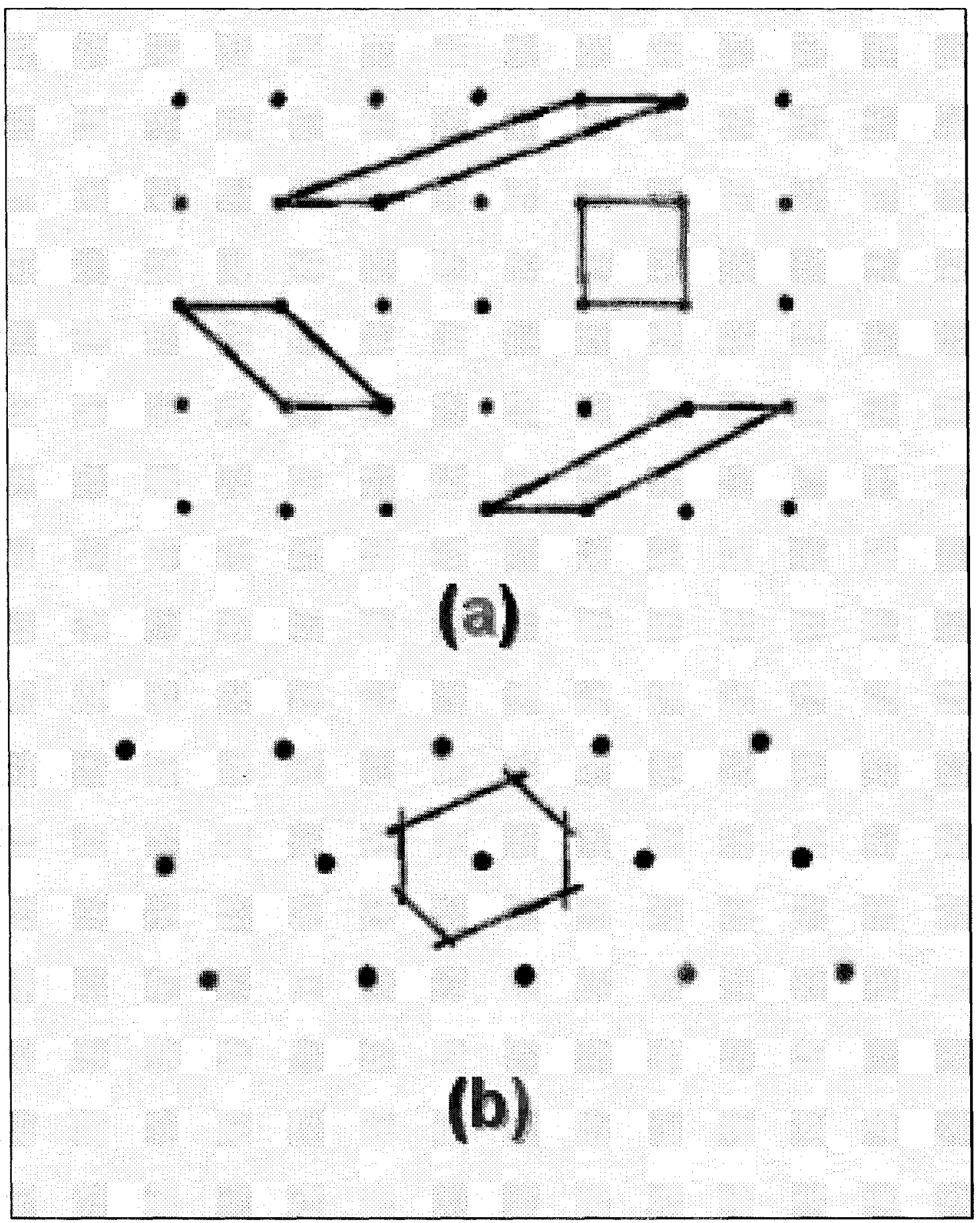

Figure 2.1. (a) primitive cells of arbitrary shape in 2D crystals and (b) Wigner-Seitz primitive cell. 


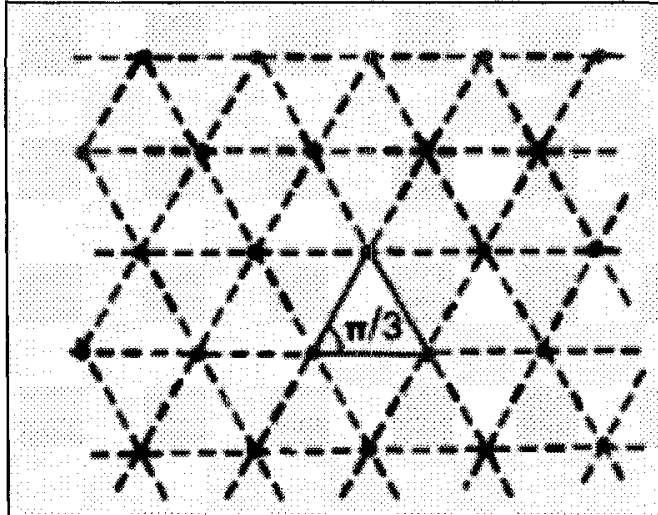

(a)

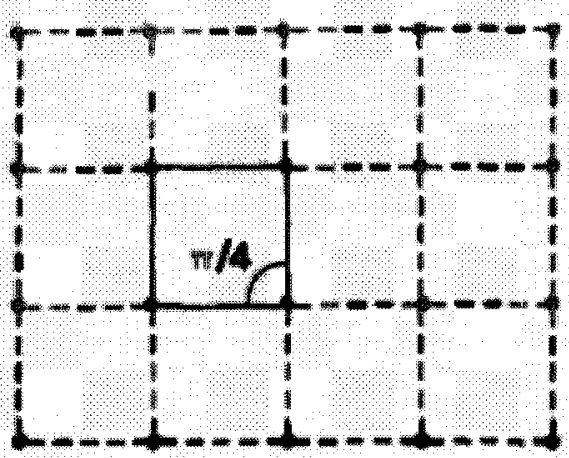

(b)

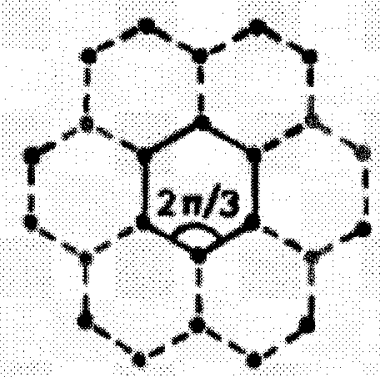

(c)

Figure 2.2. Primitive cells can be rotated through a special angle to construct an entire crystal. (a) Triangular cell, (b) square cell and (c) hexagonal cell.

The famous Bragg condition,

$$
2 d \sin \theta=n \lambda
$$

where $d$ is the lattice plane separation, $\theta$ is the incident angle measured from the plane, and $n$ is an integral number of wavelengths $\lambda$, can be re-expressed in terms of the reciprocal lattice vector $\mathbf{K}$,

$$
\mathbf{K}=\mathbf{k}^{\prime}-\mathbf{k} \text {. }
$$


where $\mathbf{k}^{\prime}$ is the outgoing wavevector and $\mathbf{k}$ is the incoming wavevector of some wavefront. This is known as the Laue condition. If the scattering is elastic ${ }^{*}$ then the condition that $|\mathbf{k}|=\left|\mathbf{k}^{\prime}-\mathbf{K}\right|$ leads to

$$
\mathbf{k} \cdot \mathbf{K}=\frac{1}{2}|\mathbf{K}|
$$

which means that an incident wavevector will satisfy the Laue condition if and only if the tip of the vector lies in a plane that is the orthogonal bisector of a line joining the origin of $k$-space to a reciprocal lattice point $\mathbf{K}$.

Such $k$-space planes are called Bragg planes. When these planes enclose a volume or area, these enclosures are called Brillouin zones. The Brillouin zones can be thought of as Wigner-Seitz cells in reciprocal space.

\subsubsection{Quasicrystal Lattices}

The previous terminology about Bragg planes and Brillouin zones are made possible because one can properly define a reciprocal space from a crystalline direct space. The crystalline direct space is possible because the primitive cell can tile all of space using the appropriate rotation point groups. This leads to the ability to describe the lattice as sheets or lines of planewaves.

There are "forbidden" primitive cell shapes because when they are rotated through their angles, they cannot tile all of space or they overlap, as shown in figure 2.3 . What is required to tile all of space is more than one shape of tile. A famous example of this is known as Penrose tiling, shown in figure 2.4 .

\footnotetext{
"Energy is preserved in the scattering, but direction is not.
} 


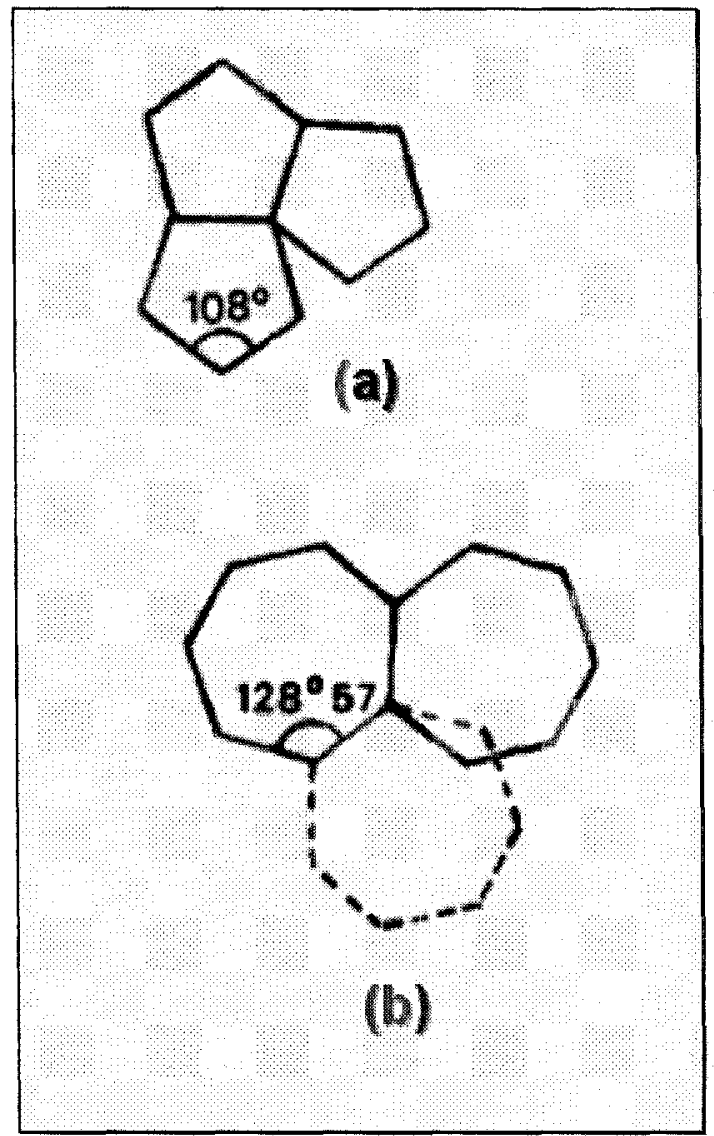

Figure 2.3. Forbidden rotations lead to an inability to tile all of space.

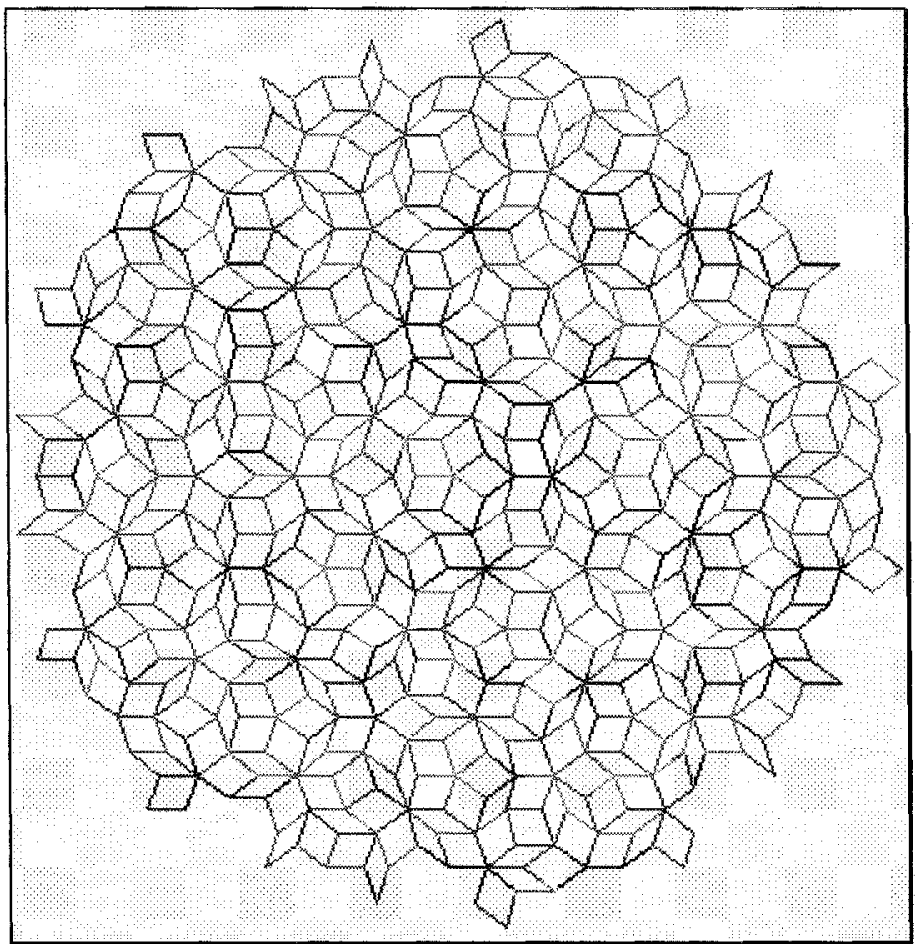

Figure 2.4. Penrose tiling. 
These type of lattices and structures were once thought to be only a mathematical curiosity up until 1984 when Dan Schetmann discovered naturally occurring structures in quenched metals [7]. A single grain of the AlCuFe alloy is shown in figure 2.5. The shape is a dodecahedron.

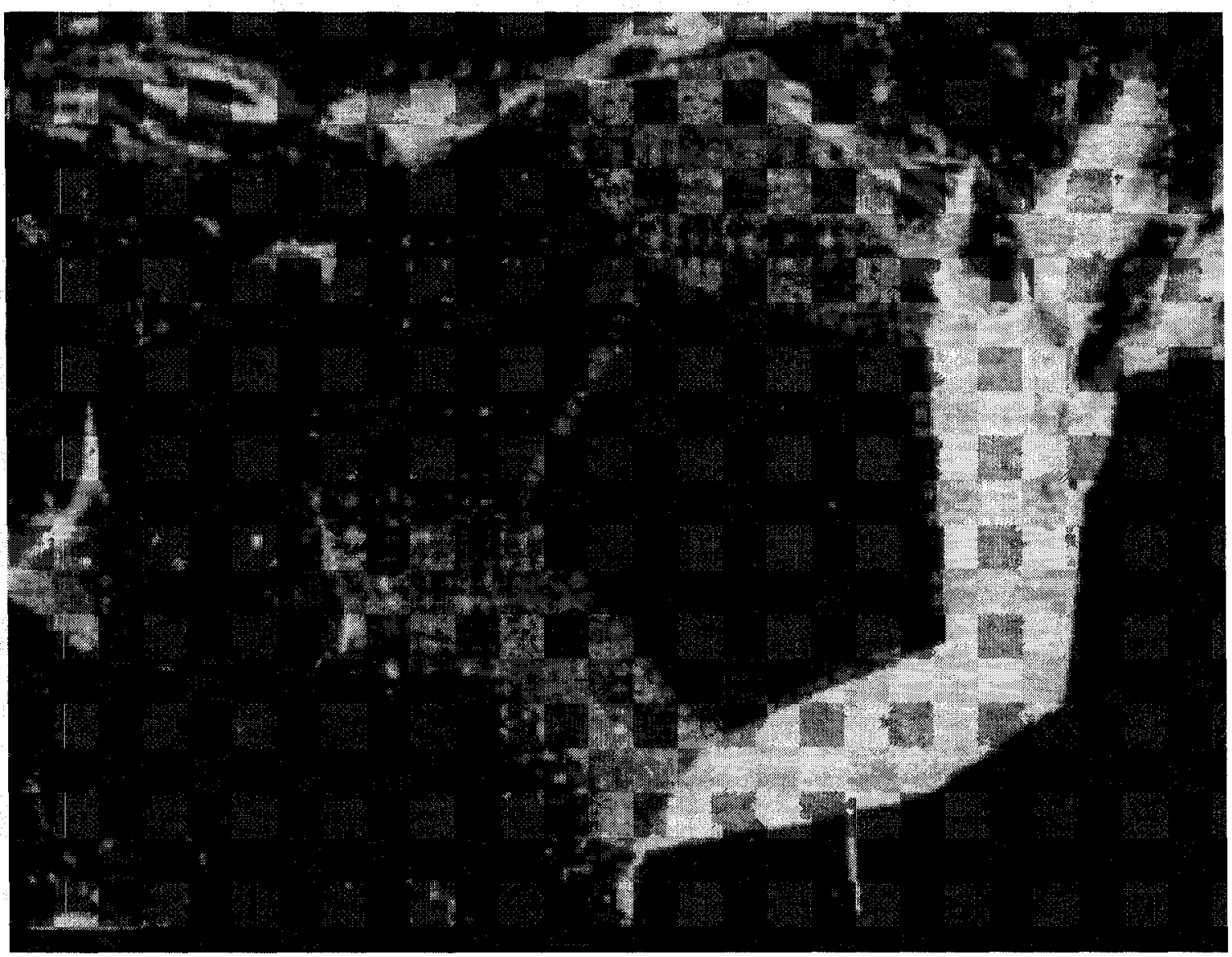

Figure 2.5. Scanning electron microscope image of quasicrystal 3D lattice of AlCuFe alloy. Dotted pentagon is drawn to highlight physical pentagon.

The previous tilings are called quasicrystals because they are not translationally symmetric like crystals, but they are also not disordered, like amorphous materials. They do have long-range order like regular crystals. One way of describing quasicrystals is that they are crystals with an irrational number for a lattice constant [12].

With no translational symmetry, the question that arises is, do the other symmetries, namely the rotational symmetries, play a role in the dynamics of what occurs within them. In this thesis, the quasicrystal structure influence within electrodynamics is 
studied. The next few sections will only deal with theories for crystalline lattices in order to introduce concepts that will be applied approximately to quasicrystalline lattices.

\subsubsection{Form Factors}

If a basis of scatterers are now attached to each lattice point, the phase difference between waves scattered at different points within the primitive cell will lead to constructive or destructive interference. The amplitudes of two scattered rays will differ by a factor $e^{i \mathbf{K} \cdot\left(\mathbf{d}_{i}-\mathbf{d}_{j}\right)}$, where $\mathbf{d}_{i}$ and $\mathbf{d}_{j}$ are position of the scatterers within the primitive cell. The net ray scattered by the entire primitive cell is the sum of the individual rays,

$$
S_{\mathbf{K}}=\sum_{j=1}^{n} e^{i \mathbf{K} \cdot \mathbf{d}_{j}}
$$

where $S_{\mathrm{K}}$ is called the geometric structure factor. It is a measure of the extent to which the interference of waves scattered from identical scatterers within the basis can diminish the intensity of the Bragg peak associated with the reciprocal lattice vector $K$. If the scatterers of the basis are not identical the structure factor assumes the form,

$$
S_{\mathbf{K}}=\sum_{j=1}^{n} f_{j}(\mathbf{K}) e^{i \mathbf{K} \cdot \mathbf{d}_{j}}
$$

where $f_{j}$ is known as the form factor and it is associated with the Bragg reflection given by the reciprocal lattice vector $\mathbf{K}$. It is proportional to the Fourier transform of the scatterer distribution of the corresponding point within the primitive cell.

\footnotetext{
${ }^{*}$ Different material properties or different shapes and sizes, or both.
} 


\subsubsection{Bloch's Theorem}

The final concept from solid-state physics required for deriving the theory for analyzing PhCs and PhQs is Bloch's theorem. Proof of Bloch's theorem is found in Appendix II. It was this theorem that established the concept that an electron in a solid can be represented as an amplitude modulated free electron wave packet,

$$
\psi_{\mathbf{k}}(\mathbf{r})=e^{i \mathbf{k} \cdot \mathbf{r}} u_{\mathbf{k}}(\mathbf{r})
$$

where $\mathbf{k}$ is one of the allowed primitive cell wavevectors, $\mathbf{r}$ is a position vector, $\psi_{\mathbf{k}}(\mathbf{r})$ is the amplitude to find an electron with wavevector $\mathbf{k}$ and position $\mathbf{r}, e^{i \mathbf{k} \cdot \mathbf{r}}$ is the freespace electron amplitude and $u_{\mathbf{k}}(\mathbf{r})$ is a function that modulates this free-space amplitude by the periodicity of the crystal. The form of $u_{\mathbf{k}}(\mathbf{r})$ has the periodicity of the crystal,

$$
u_{\mathrm{k}}(\mathbf{r})=u_{\mathrm{k}}(\mathbf{r}+\mathbf{R}) .
$$

An interesting note about this is the following:

$$
\begin{aligned}
\psi_{\mathbf{k}}(\mathbf{r}+\mathbf{R}) & =e^{i \mathbf{k} \cdot(\mathbf{r}+\mathbf{R})} u_{\mathbf{k}}(\mathbf{r}+\mathbf{R}) \\
& =e^{i \mathbf{k} \cdot \mathbf{R}} e^{i \mathbf{k} \cdot \mathbf{r}} u_{\mathbf{k}}(\mathbf{r}) \\
& =e^{i \mathbf{k} \cdot \mathbf{R}} \psi_{\mathbf{k}}(\mathbf{r}) .
\end{aligned}
$$

This result shows that a discrete symmetry operation on the wave function such as a spatial translation via a lattice vector is equivalent to multiplication of the wave function by a complex exponential. This result is similar to continuous transformations, as will be shown later in this thesis. In addition, this result lends itself to the explanation that the discrete translation operator commutes with the equation-of-motion operator. This implies that these two operators possess a complete set of common eigenvectors. However, the point that must be made here is that this result is non-rigorous from a mathematical point of view. It is non-rigorous from a group theoretic point of view 
because discrete symmetries cannot be described via Lie algebra. This point is being made now because later when only rotational symmetries are available in the structure a similar approach will be asserted, namely that the rotational symmetry gives rise to a Bloch-type mode about the center of rotation. This rotational Bloch equivalent looks like a defect mode but it is fundamentally different.

\subsection{Bandstructure Calculations}

\subsubsection{Determinant Method}

The concept of a bandstructure may be illustrated through the following $1 \mathrm{D}$ example. One takes equation 2.20 , and expresses the relative dielectric constant in terms of the reciprocal lattice and electric field in Fourier space,

$$
\begin{gathered}
\varepsilon_{r}(\mathbf{r})=\sum_{\mathbf{K}} \varepsilon_{\mathbf{K}} e^{-i \mathbf{K} \cdot \mathbf{r}} \\
\mathbf{E}(\mathbf{r})=\int d^{3} k \mathbf{A}(\mathbf{k}) e^{-i \mathbf{k} \cdot \mathbf{r}} .
\end{gathered}
$$

Substituting equations 2.31 and 2.32 into 2.20 one arrives at,

$$
\int d^{3} k[\mathbf{k} \times \mathbb{k} \times \mathbf{A}(\mathbf{k})] e^{-i \mathbf{k} \cdot \mathbf{r}}+\frac{\omega^{2}}{c^{2}} \sum_{\mathbf{K}} \int d^{3} k \varepsilon_{\mathbf{K}} \mathbf{A}(\mathbf{k}-\mathbf{K}) e^{-i \mathbf{k} \cdot \mathbf{r}}=0
$$

This relation is only true if

$$
\mathbf{k} \times[\mathbf{k} \times \mathbf{A}(\mathbf{k})]+\frac{\omega^{2}}{c^{2}} \sum_{\mathbf{K}} \varepsilon_{\mathbf{K}} \mathbf{A}(\mathbf{k}-\mathbf{K})=0 .
$$

Taking a $1 \mathrm{D}$ example, the relation becomes,

$$
k^{2} A(k)-\frac{\omega^{2}}{c^{2}} \sum_{l} \varepsilon_{l} A(k-l g)=0
$$


where $K \rightarrow \lg$ and $g=2 \pi / \Lambda$, where $\Lambda$ is the lattice constant and $l=0, \pm 1, \pm 2, \pm 3, \ldots$ Expanding 2.35 for $A(k)$ and $A(k-g)$,

$$
\begin{aligned}
A(k) & =\frac{\omega^{2} / c^{2}}{\left(k^{2}-\omega^{2} / c^{2}\right)}\left[\varepsilon_{1} A(k-g)+\varepsilon_{-1} A(k+g)+\cdots\right] \\
A(k-g) & =\frac{\omega^{2} / c^{2}}{\left((k-g)^{2}-\omega^{2} / c^{2}\right)}\left[\varepsilon_{1} A(k-2 g)+\varepsilon_{-1} A(k)+\cdots\right] .
\end{aligned}
$$

If the first two terms of the series are taken (as a lincar approximation), then

$$
\begin{gathered}
\left(k^{2}-\frac{\omega^{2}}{c^{2}} \varepsilon_{0}\right) A(k)-\frac{\omega^{2}}{c^{2}} \varepsilon_{l} A(k-g)=0 \\
-\frac{\omega^{2}}{c^{2}} \varepsilon_{-1} A(k)+\left[(k-g)^{2}-\frac{\omega^{2}}{c^{2}} \varepsilon_{0}\right] A(k-g)=0 .
\end{gathered}
$$

When solving two coupled equations, a necessary condition for a non-trivial solution is that the determinant vanishes:

$$
\left|\begin{array}{cc}
k^{2}-\frac{\omega^{2}}{c^{2}} \varepsilon_{0} & -\frac{\omega^{2}}{c^{2}} \varepsilon_{1} \\
-\frac{\omega^{2}}{c^{2}} \varepsilon_{-1} & (k-g)^{2}-\frac{\omega^{2}}{c^{2}} \varepsilon_{0}
\end{array}\right|=0
$$

or

$$
\left[k^{2}-\frac{\omega^{2}}{c^{2}} \varepsilon_{0}\right]\left[(k-g)^{2}-\frac{\omega^{2}}{c^{2}} \varepsilon_{0}\right]-\left[\frac{\omega^{2}}{c^{2}}\left|\varepsilon_{1}\right|\right]^{2}=0
$$

Equation 2.41 becomes the new dispersion relation of a Bloch wave propagating through material with a periodically modulated dielectric. Figure 2.6 is a plot of a 1D dispersion curve. 


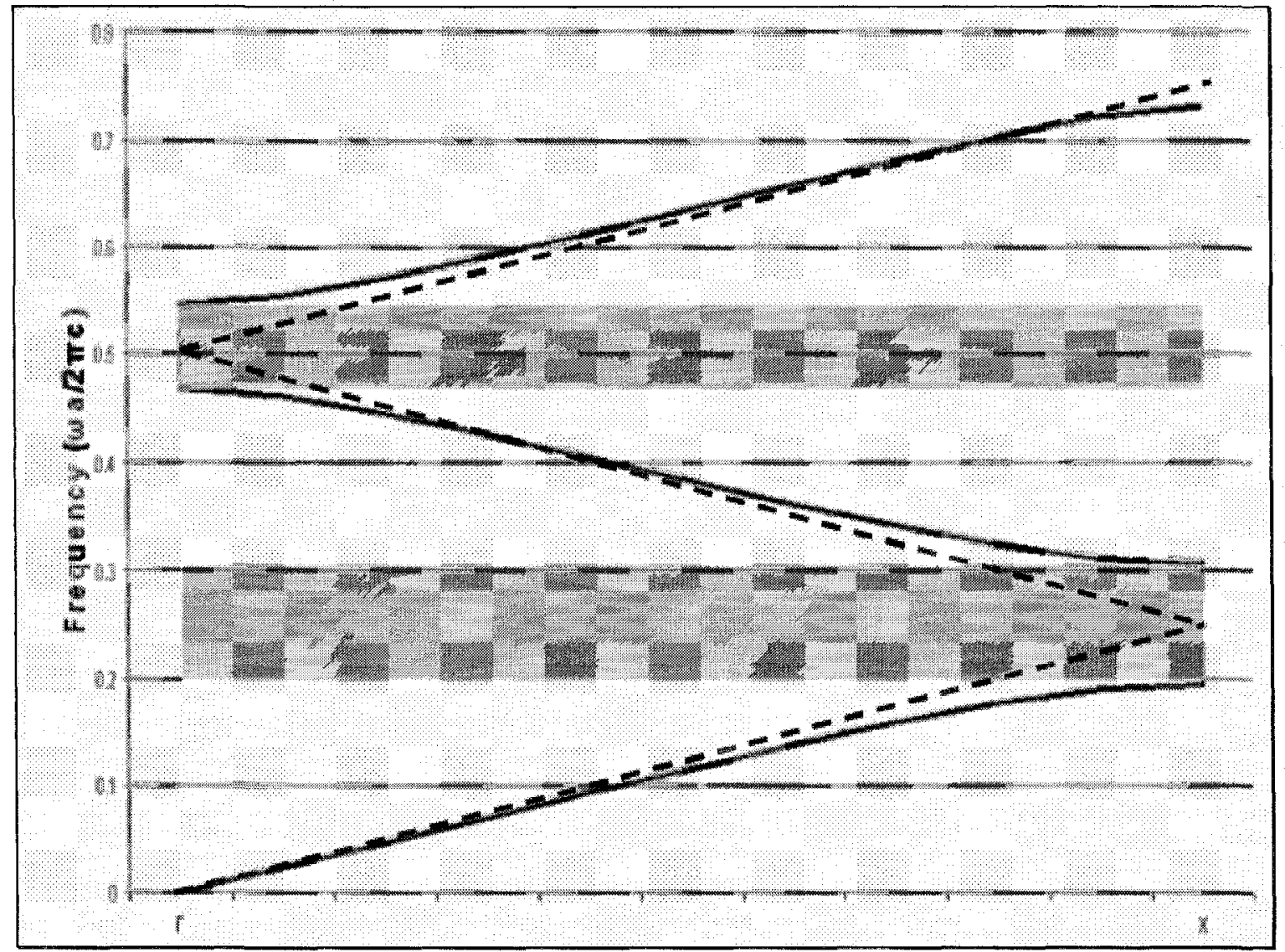

Figure 2.6. Dispersion relation for 1D periodic structure. Dashed line is the dispersion relation in a vacuum.

When the dispersion relation is plotted like this, this plot is called the optical bandstructure of the material. The dispersion relation in optics and the $E, p$-diagrams of solid-state physics are of the same nature.

\subsubsection{Planewave Analysis: Diagonalization Method.}

In more than one dimension, the determinant method used in the last section to obtain the dispersion relation becomes complicated. Another approach is to generate a matrix that describes the electrodynamics in the lattice and then diagonalize the matrix. This is equivalent to solving the eigenvalue problem. To obtain this matrix, one takes equations 2.20 and 2.21 and expresses the inverse dielectric in terms of the reciprocal lattice, 


$$
\frac{1}{\varepsilon(\mathbf{r})}=\sum_{\mathbf{K}} \kappa(\mathbf{K}) e^{i \mathbf{K} \cdot \mathbf{r}}
$$

and because the dielectric constant is real then $\kappa(-\mathbf{K})=\kappa^{*}(\mathbf{K})$. Due to the translational symmetry, one can express the field quantities as Bloch modes,

$$
\begin{aligned}
& \mathbf{E}(\mathbf{r})=\mathbf{E}_{n \mathbf{k}}(\mathbf{r})=\mathbf{u}_{\mathbf{k} n}(\mathbf{r}) e^{i \mathbf{k} \cdot \mathbf{r}} \\
& \mathbf{H}(\mathbf{r})=\mathbf{H}_{n \mathbf{k}}(\mathbf{r})=\mathbf{v}_{\mathbf{k} n}(\mathbf{r}) e^{i \mathbf{k} \cdot \mathbf{r}}
\end{aligned}
$$

where $\mathbf{u}_{\mathbf{k} n}(\mathbf{r})$ and $\mathbf{v}_{\mathrm{k} n}(\mathbf{r})$ are the Bloch vector functions that have the periodicity of the translationally symmetric structure. This is where planewave analysis gets its name: the field quantities are expressed in terms of amplitude-modulated planewaves. One now expresses the field components in the $k$-space,

$$
\begin{aligned}
\mathbf{E}_{\mathbf{k} n}(\mathbf{r}) & =\sum_{\mathbf{K}} \mathbf{E}_{\mathbf{k} n}(\mathbf{K}) \exp \{i(\mathbf{k}+\mathbf{K}) \cdot \mathbf{r}\} \\
\mathbf{H}_{\mathbf{k} n}(\mathbf{r}) & =\sum_{\mathbf{K}} \mathbf{H}_{\mathbf{k} n}(\mathbf{K}) \exp \{i(\mathbf{k}+\mathbf{K}) \cdot \mathbf{r}\} .
\end{aligned}
$$

Incorporating these into the equations of motion gives,

$$
\begin{aligned}
& -\sum_{\mathbf{K}^{\prime}} \kappa\left(\mathbf{K}-\mathbf{K}^{\prime}\right)\left(\mathbf{k}-\mathbf{K}^{\prime}\right) \times\left\{\left(\mathbf{k}-\mathbf{K}^{\prime}\right) \times \mathbf{E}_{\mathbf{k} n}\left(\mathbf{K}^{\prime}\right)\right\}=\frac{\omega_{\mathbf{k} n}^{2}}{c^{2}} \mathbb{E}_{\mathbf{k} n}(\mathbf{K}) \\
& -\sum_{\mathbf{K}^{\prime}}\left(\mathbf{k}-\mathbf{K}^{\prime}\right) \times\left\{\kappa\left(\mathbf{K}-\mathbf{K}^{\prime}\right)\left(\mathbf{k}-\mathbf{K}^{\prime}\right) \times \mathbf{H}_{\mathbf{k} n}\left(\mathbf{K}^{\prime}\right)\right\}=\frac{\omega_{\mathbf{k} n}^{2}}{c^{2}} \mathbb{H}_{\mathbf{k} n}\left(\mathbf{K}^{\prime}\right)
\end{aligned}
$$

where $\nabla \rightarrow \mathbf{k}-\mathbf{K}$ occurs because of the Fourier transform. This is similar to what happens in quantum mechanics in which momentum is a spatial derivative in position space. 


\subsubsection{Planewave Analysis: Variational Problem.}

Equations 2.47 and 2.48 are the starting equations for numerical methods that take a matrix and diagonalizes it to fully solve the eigenvalue problem. However, this method can be quite computationally expensive to solve. Another method is to iteratively minimize an expectation expression. One can establish an electromagnetic variational problem similar to that in solid-state physics when trying to obtain the $E$,p-diagram for some material. This variation expression is,

$$
\left\langle L_{H}\right\rangle=\frac{\left\langle\mathbf{H}\left|L_{H}\right| \mathbf{H}\right\rangle}{\langle\mathbf{H} \mid \mathbf{H}\rangle}
$$

where $\langle\mid\rangle$ is the Dirac notation and, for vector quantities, represents,

$$
\langle\mathbf{F}(\mathbf{r}) \mid \mathbf{G}(\mathbf{r})\rangle=\int d^{3} r \mathbf{F}^{*}(\mathbf{r}) \cdot \mathbf{G}(\mathbf{r})
$$

where $\mathbf{F}(\mathbf{r})$ and $\mathbf{G}(\mathbf{r})$ are arbitrary vector field quantities. Applying the convention of 2.50 to relation 2.49 yields:

$$
\begin{gathered}
\left\langle\mathbf{H}\left|L_{H}\right| \mathbf{H}\right\rangle=\int d^{3} r \frac{1}{\varepsilon(\mathbf{r})} \nabla \times\left.\mathbf{H}(\mathbf{r})\right|^{2}=\frac{\omega^{2}}{c^{2}} \int d^{3} r \frac{1}{\varepsilon(\mathbf{r})} \mid D(\mathbf{r})^{2} \\
\langle\mathbf{H} \mid \mathbf{H}\rangle \equiv \int d^{3} r \mathbf{H}^{*}(\mathbf{r}) \cdot \mathbf{H}^{*}(\mathbf{r})=\int d^{3} r|\mathbf{H}(\mathbf{r})|^{2}
\end{gathered}
$$

where $\mathbf{D}(\mathbf{r})$ is the electric flux density introduced in equation 2.5 . In order to minimize relation 2.49 , two conditions must be met. Firstly, the curl of the magnetic field must be small, which means that the field should vary slowly (ie. analogous to having low kinetic energy in quantum mechanics). Secondly, the curl of the magnetic field, which is the electric field, should be concentrated in regions of high dielectric (ie. analogous to having low potential energy in quantum mechanics). 
In order to obtain the bandstructure in this way, the modes that are solved for when minimizing relation 2.49 need to be orthogonal to each other. That is, eigenmodes with different associated eigenvalues must be orthogonal. An operator that admits this condition is known as Hermitian. One can determine if an operator is Hermitian if the following condition is met,

$$
\langle\mathbf{F}(\mathbf{r})|L| \mathbf{G}(\mathbf{r})\rangle=\langle\mathbf{F}(\mathbf{r}) \mid L \mathbf{G}(\mathbf{r})\rangle=\langle L \mathbf{F}(\mathbf{r}) \mid \mathbf{G}(\mathbf{r})\rangle
$$

where $\mathbf{F}(\mathbf{r})$ and $\mathbf{G}(\mathbf{r})$ are arbitrary vector field quantities, $L$ is some operator and the second equality denotes that the operator in between the vector field states is a Hermitian operator.

One will notice in relation 2.49 that the operator is the magnetic field operator $L_{\mathrm{H}}$ and not electric field operator $L_{\mathrm{E}}$. The reason for this is that $L_{\mathrm{H}}$ is Hermitian but $L_{\mathrm{E}}$ is not. The electric field operator can also be made Hermitian if its form is altered. If the electric field is expressed as,

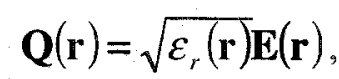

and 2.20 is expressed as,

$$
L_{\mathrm{Q}} \mathbf{Q}(\mathbf{r})=\frac{1}{\sqrt{\varepsilon_{r}(\mathbf{r})}} \nabla \times\left\{\nabla \times \frac{1}{\sqrt{\varepsilon_{r}(\mathbf{r})}} \mathbb{E}(\mathbf{r})\right\}
$$

then $L_{\mathrm{Q}}$ can be shown to be Hermitian.

As an example of the above minimization, one has a diamond lattice structure, where the unit cell is shown in figure 2.7. This crystal is represented mathematically in 2.42. One then solves for the eigenmodes by minimizing relation 2.49 , and ultimately the dispersion curve shown in figure 2.8 is obtained. 


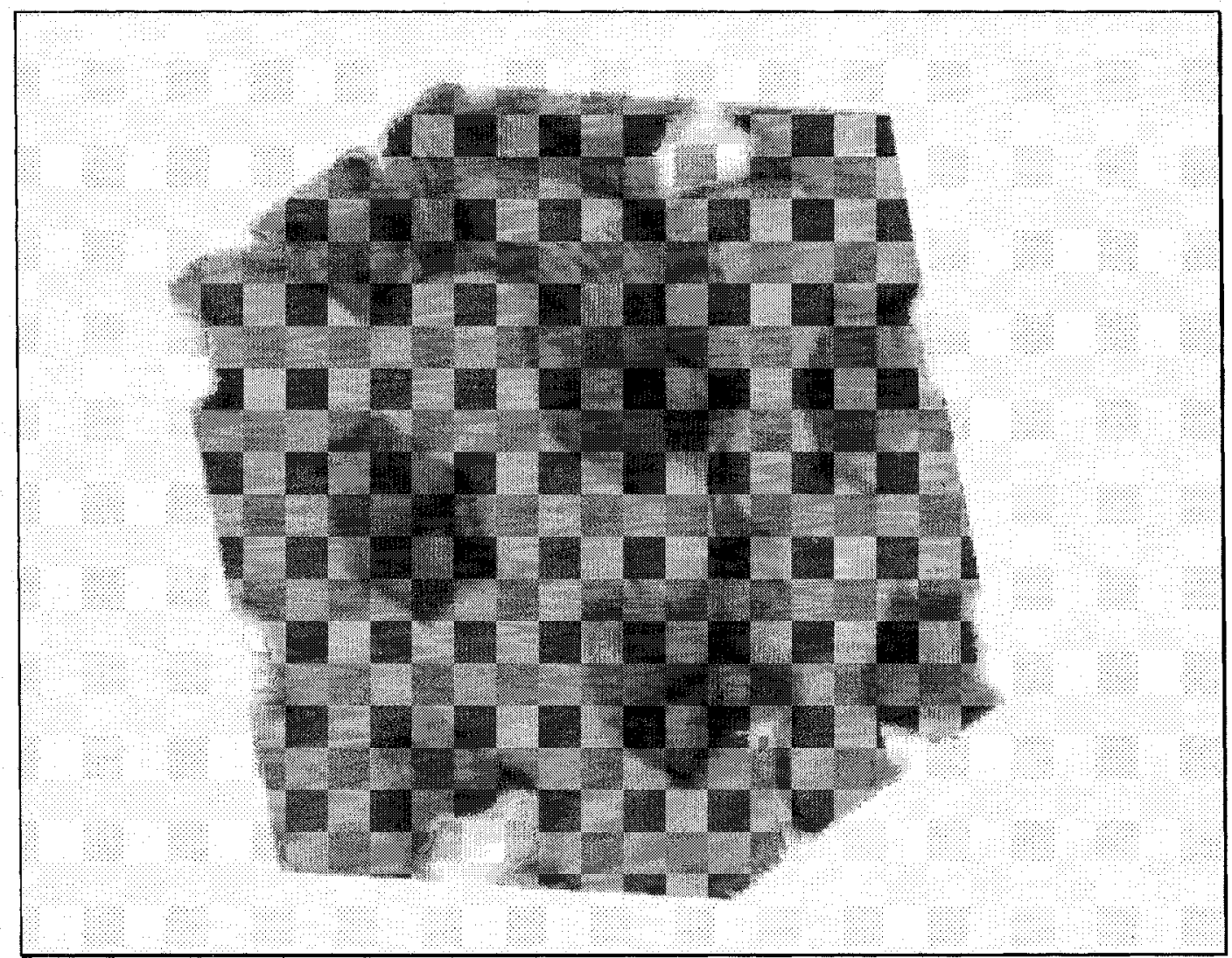

Figure 2.7. Unit cell of diamond lattice structure.

Notice that this structure has a band gap, which is represented by the dark band in the figure. The abscissa in figure 2.8 are the directions in the Brillouin zone of the reciprocal lattice. The diamond lattice is actually a face-centered cubic structure with two spheres of some material per basis. The Brillouin zone of an FCC structure is shown in figure 2.9 .

All the above work is for translationally periodic 3D structures. This thesis is about analyzing 2D PhQ structures computationally and physically. Nonetheless, the mathematical method above is also applicable to $2 \mathrm{D}$ structures. The only change is that when one introduces the 2D plane where all the dielectric variations are, all the optical modes can be categorized in two polarizations: the electric field in the direction of the normal to the $2 \mathrm{D}$ plane, and the electric field in the $2 \mathrm{D}$ plane. 


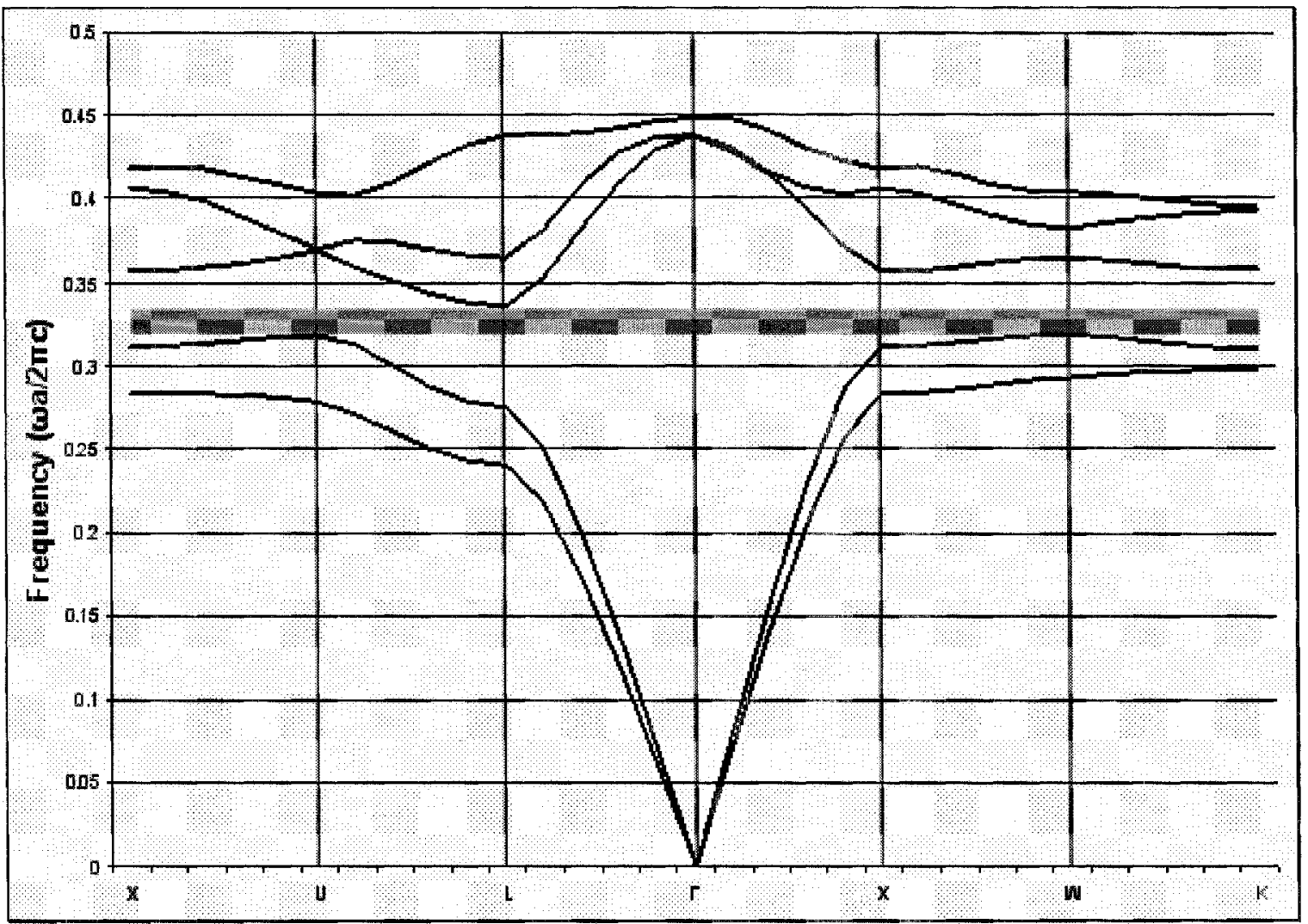

Figure 2.8. Dispersion curve obtained via matrix diagonalization. Shaded region represents a band gap.

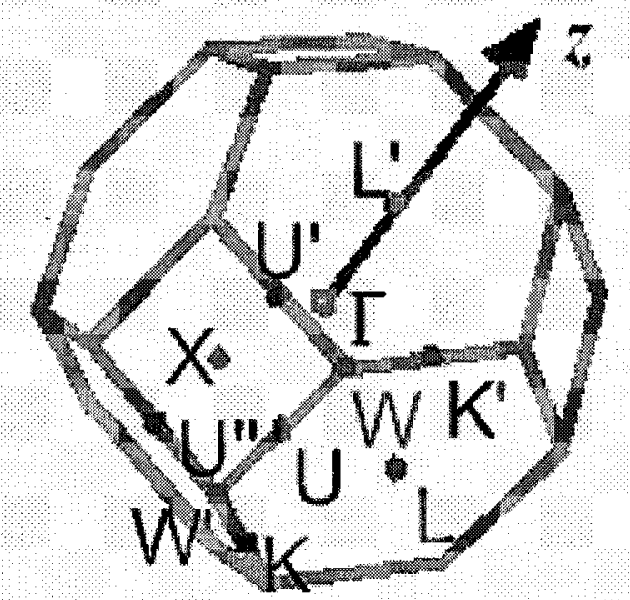

Figure 2.9. Brillouin zone for FCC structure. 


\subsubsection{Planar Structures}

For 2D translationally symmetric crystals, the eigenvalue equations become much simpler. A 2D crystal is confined to the $x y$-plane and is infinite in extent in the $z$ direction, as shown in figure 2.10 .

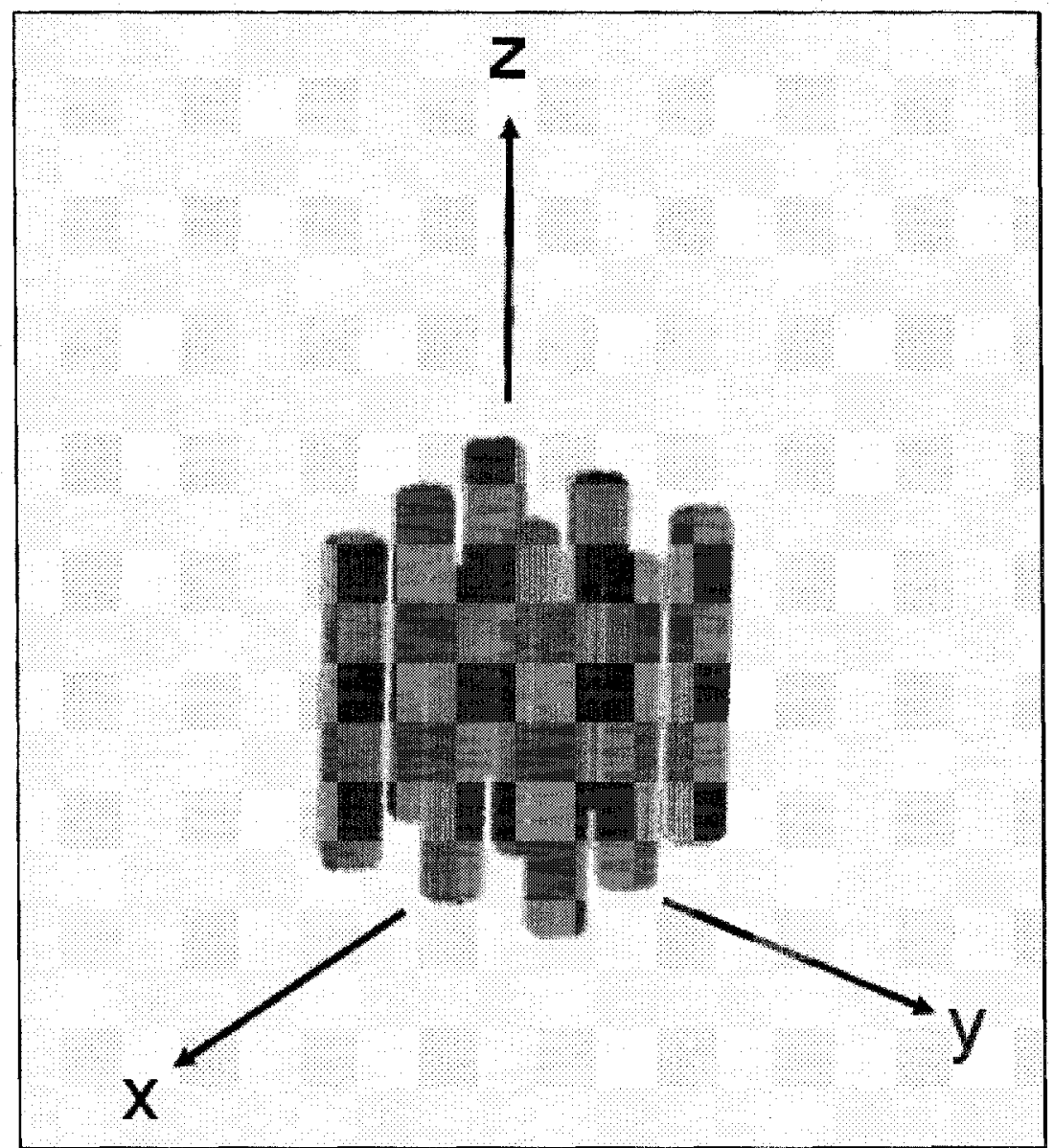

Figure 2.10. Two-dimensional crystal. Square lattice with circular basis.

Figure 2.11 gives an example of the dielectric variation in the 2D plane of a $\mathrm{PhC}$ and a $\mathrm{PhQ}$. The $\mathrm{PhC}$ is a hexagonal array of dielectric scatterers and the PhQ is a 12 -fold lattice of dielectric scatterers as well. The geometry of the dielectric scatterers in the PhC are circles, while the geometry of the dielectric scatterers in the PhQ vary, but not in an arbitrary manner. Usually in the literature, one will find $\mathrm{PhQ}$ structures with 
conventional dielectric scatterer geometries, such as circular rods, as shown in figure

2.12.

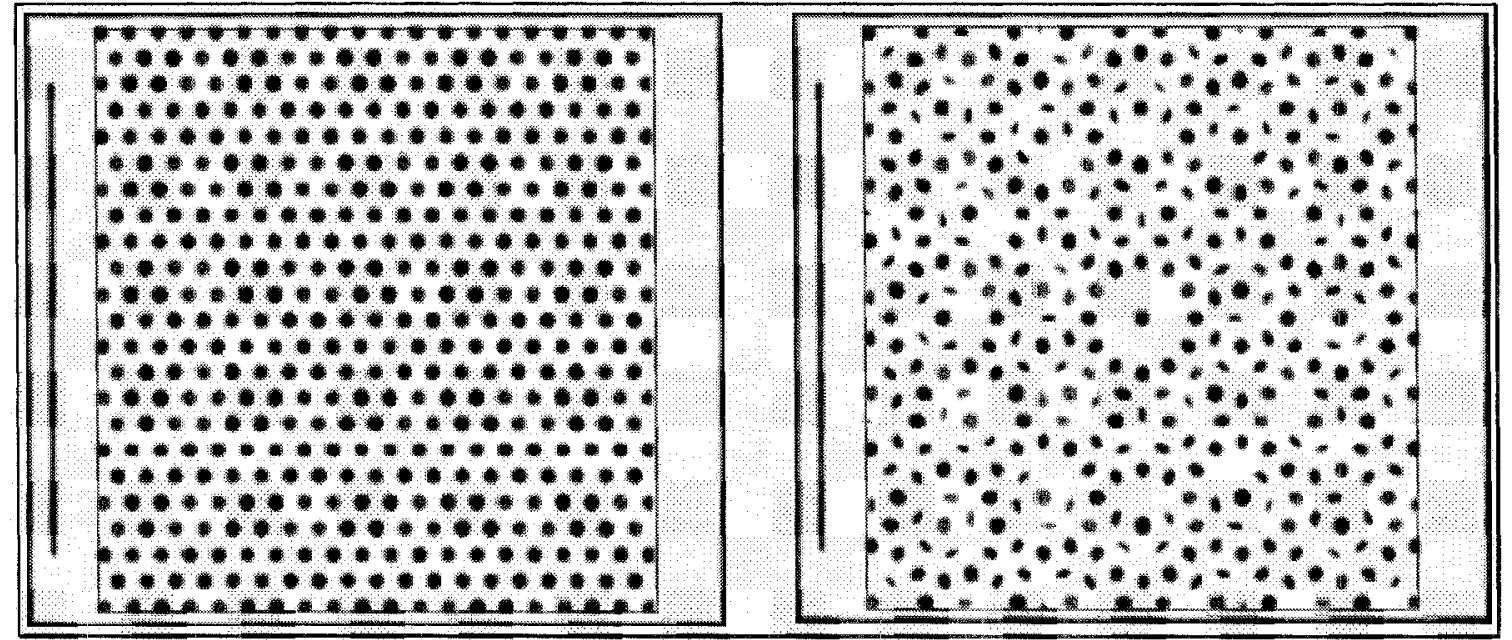

Figure 2.11. Photonic crystal and photonic quasicrystal.

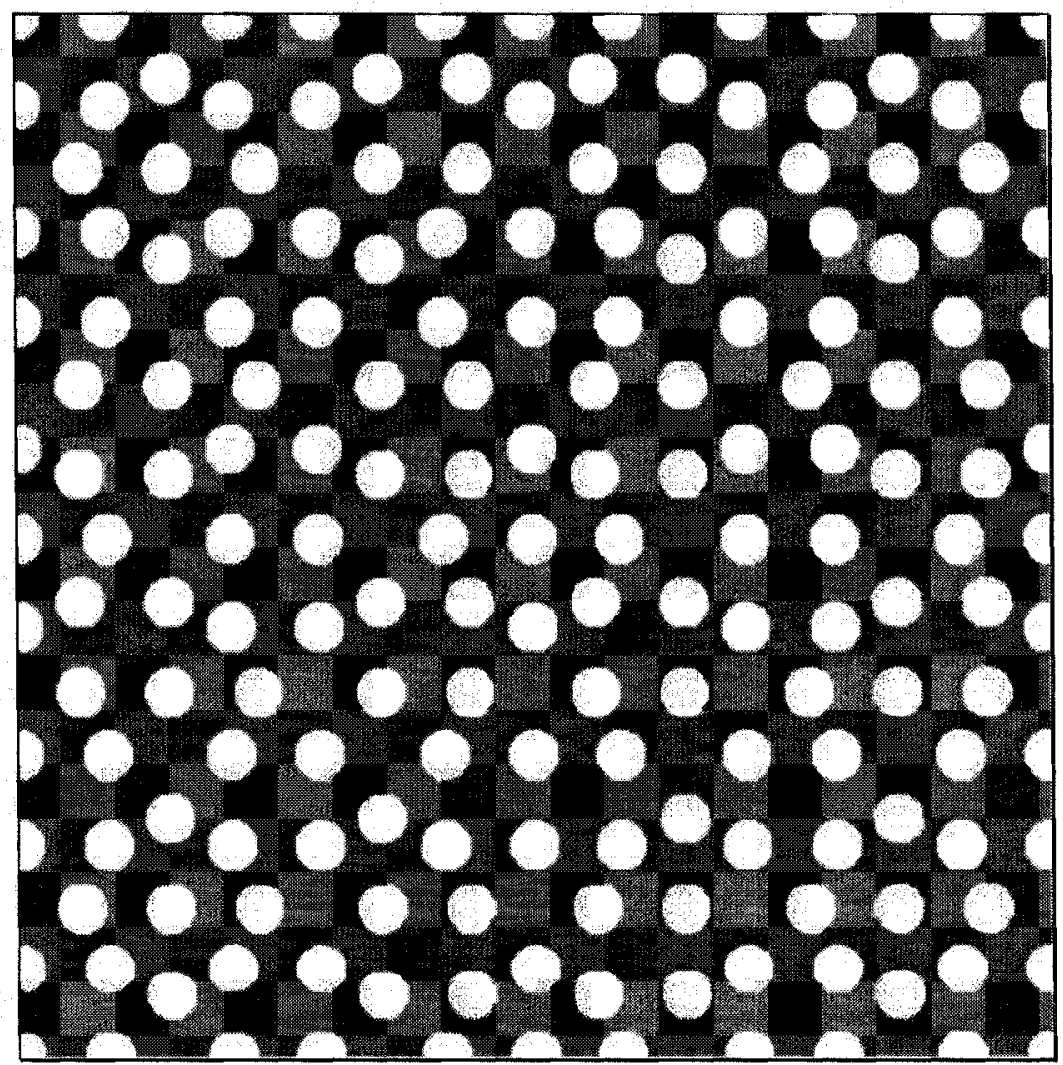

Figure 2.12. Octagonal PhQ using circular rods [8].

It will be shown that PhQs also admit photonic bandstructures just like PhCs. In addition, when the shape of the dielectric scatterers are non-conventional, new features start to appear and it is these features that led the author to the concept of crystal angular 
momentum. Before discussing these features and the bandstructure of PhQs, the theory for $2 \mathrm{D}$ PhCs will be reviewed.

In 2D PhCs, the Maxwell's equations are decoupled into two independent sets of equations; the first set is:

$$
\begin{gathered}
\frac{\partial}{\partial y} E_{z}(\boldsymbol{\rho}, t)=-\mu_{0} \frac{\partial}{\partial t} H_{x}(\boldsymbol{\rho}, t) \\
\frac{\partial}{\partial x} E_{z}(\boldsymbol{\rho}, t)=\mu_{0} \frac{\partial}{\partial t} H_{y}(\boldsymbol{\rho}, t) \\
\frac{\partial}{\partial x} H_{y}(\boldsymbol{\rho}, t)-\frac{\partial}{\partial y} H_{x}(\boldsymbol{\rho}, t)=\varepsilon_{0} \varepsilon_{r}(\boldsymbol{\rho}) \frac{\partial}{\partial t} E_{z}(\boldsymbol{\rho}, t),
\end{gathered}
$$

and the second set is:

$$
\begin{gathered}
\frac{\partial}{\partial y} H_{z}(\boldsymbol{\rho}, t)=\varepsilon_{0} \varepsilon_{r}(\boldsymbol{\rho}) \frac{\partial}{\partial t} E_{x}(\boldsymbol{\rho}, t) \\
\frac{\partial}{\partial x} H_{z}(\boldsymbol{\rho}, t)=-\varepsilon_{0} \varepsilon_{r}(\boldsymbol{\rho}) \frac{\partial}{\partial t} E_{y}(\boldsymbol{\rho}, t) \\
\frac{\partial}{\partial x} E_{y}(\boldsymbol{\rho}, t)-\frac{\partial}{\partial y} E_{x}(\boldsymbol{\rho}, t)=-\mu_{0} \frac{\partial}{\partial t} H_{z}(\boldsymbol{\rho}, t),
\end{gathered}
$$

where $\rho$ is the $2 \mathrm{D}$ position vector in the $x y$-plane. One can then obtain two wave equations in terms of $E_{z}(\boldsymbol{\rho}, t)$ and $H_{z}(\boldsymbol{\rho}, t)$.

$$
\begin{gathered}
\frac{1}{\varepsilon(\boldsymbol{\rho})}\left\{\frac{\partial^{2}}{\partial x^{2}}+\frac{\partial^{2}}{\partial y^{2}}\right\} E_{z}(\boldsymbol{\rho}, t)=\frac{1}{c^{2}} \frac{\partial^{2}}{\partial t^{2}} E_{z}(\boldsymbol{\rho}, t) \\
\left\{\frac{\partial}{\partial x} \frac{1}{\varepsilon(\rho)} \frac{\partial}{\partial x}+\frac{\partial}{\partial y} \frac{1}{\varepsilon(\boldsymbol{\rho})} \frac{\partial}{\partial y}\right\} H_{z}(\boldsymbol{\rho}, t)=\frac{1}{c^{2}} \frac{\partial^{2}}{\partial t^{2}} H_{z}(\boldsymbol{\rho}, t) .
\end{gathered}
$$

As before, one seeks time-harmonic solutions, 


$$
\begin{aligned}
& E_{z}(\boldsymbol{\rho}, t)=E_{z}(\boldsymbol{\rho}) e^{-i \omega t}, \\
& H_{z}(\boldsymbol{\rho}, t)=H_{z}(\boldsymbol{\rho}) e^{-i \omega t} .
\end{aligned}
$$

The eigenvalue equations become,

$$
\begin{aligned}
& L_{E}^{\partial D} E_{z}(\boldsymbol{\rho}, t)=\frac{1}{\varepsilon(\boldsymbol{\rho})}\left\{\frac{\partial^{2}}{\partial x^{2}}+\frac{\partial^{2}}{\partial y^{2}}\right\} E_{z}(\boldsymbol{\rho}, t)=\frac{\partial^{2}}{c^{2}} E_{z}(\boldsymbol{\rho}, t) \\
& L_{H}^{2 D} H_{s}(\boldsymbol{\rho}, t)=-\left\{\frac{\partial}{\partial x \frac{1}{c(\boldsymbol{\rho})} \frac{\partial}{\partial x}}+\frac{\partial}{\partial y} \frac{1}{c(\boldsymbol{\rho})} \frac{\partial}{\partial y}\right\} H_{z}(\boldsymbol{\rho}, t)=\frac{\omega^{2}}{c^{2}} H_{z}(\boldsymbol{\rho}, t),
\end{aligned}
$$

where $L_{H}^{2 D}$ and $L_{E}^{2 D}$ are the differential operators defined by the first equivalence. The differential operators can also be denoted as a transverse Laplacian,

$$
\begin{aligned}
& L_{E}^{2 D} E_{z}(\boldsymbol{\rho}, t)=\frac{1}{\varepsilon(\boldsymbol{\rho})} \nabla_{t}^{2} E_{z}(\boldsymbol{\rho}, t) \\
& L_{H}^{2 D} H_{z}(\boldsymbol{\rho}, t)=-\nabla_{t} \frac{1}{\varepsilon(\boldsymbol{\rho})} \nabla_{t} H_{z}(\boldsymbol{\rho}, t)
\end{aligned}
$$

where

$$
\begin{gathered}
\nabla_{t}^{2} \equiv\left\{\frac{\partial^{2}}{\partial x^{2}}+\frac{\partial^{2}}{\partial y^{2}}\right\} \\
\nabla_{t} \frac{1}{\varepsilon(\boldsymbol{\rho})} \nabla_{t} \equiv\left\{\frac{\partial}{\partial x} \frac{1}{\varepsilon(\boldsymbol{\rho})} \frac{\partial}{\partial x}+\frac{\partial}{\partial y} \frac{1}{\varepsilon(\boldsymbol{\rho})} \frac{\partial}{\partial y}\right\}
\end{gathered}
$$

Equations 2.66 and 2.67 represent two independent polarizations because of the mirror symmetry created by the plane of propagation [51]. There seems to be an inverse convention to waveguide propagation theory. In waveguide theory, if the electric field is 
transverse to the direction of propagation, the mode is denoted transverse electric (TE), while if the magnetic field is transverse to the direction of propagation, the mode is denoted transverse magnetic (TM). However, with 2D PhCs and PhQs, if the electric field is parallel to the normal of the 2D plane, then the modes are denoted TM because it is the magnetic field that is in the 2D plane. If the magnetic field is parallel to the normal of the 2D plane, then the modes are denoted TE because it is the electric field that is in the 2D plane.

As before, the field components are described in terms of planewaves in the 2D plane with the reciprocal lattice vector of the $2 \mathrm{D}$ crystal,

$$
\begin{gathered}
E_{z}(\mathbf{\rho})=E_{z, \mathbf{k}_{i} n}(\mathbf{\rho})=\sum_{\mathbf{K}_{t}} E_{z, \mathbf{k}_{i} n}\left(\mathbf{K}_{t}\right) \exp \left\{i\left(\mathbf{k}_{t}+\mathbf{K}_{t}\right) \cdot \boldsymbol{\rho}\right\}, \\
H_{z}(\mathbf{\rho})=H_{z, \mathbf{k}_{t} n}(\mathbf{\rho})=\sum_{\mathbf{K}_{t}} H_{z, \mathbf{k}_{t} n}\left(\mathbf{K}_{t}\right) \exp \left\{i\left(\mathbf{k}_{t}+\mathbf{K}_{t}\right) \cdot \boldsymbol{\rho}\right\},
\end{gathered}
$$

where $\mathbf{k}_{t}$ and $\mathbf{K}_{t}$ are the wavevector and reciprocal lattice vector in the $2 \mathrm{D}$ plane. Substituting 2.72 and 2.73 into 2.66 and 2.67 ,

$$
\begin{gathered}
\sum_{\mathbf{G}_{t}^{\prime}} \kappa\left(\mathbf{G}_{t}-\mathbf{G}_{t}^{\prime}\right)\left|\mathbf{k}_{t}+\mathbf{G}_{t}^{\prime}\right|^{2} E_{z, \mathbf{k}_{t} n}\left(\mathbf{G}_{t}^{\prime}\right)=\frac{\omega_{\mathbf{k}_{t} n}^{(\mathrm{TM}) 2}}{c^{2}} E_{z, \mathbf{k}_{t} n}\left(\mathbf{G}_{t}\right), \\
\sum_{\mathbf{G}_{t}^{\prime}} \kappa\left(\mathbf{G}_{t}-\mathbf{G}_{t}^{\prime}\right)\left(\mathbf{k}_{t}+\mathbf{G}_{t}\right) \cdot\left(\mathbf{k}_{t}+\mathbf{G}_{t}^{\prime}\right) H_{z, \mathbf{k}_{i} n}\left(\mathbf{G}_{t}^{\prime}\right)=\frac{\omega_{\mathbf{k}_{t} n}^{(\mathrm{TE}) 2}}{c^{2}} H_{z, \mathbf{k}_{t} n}\left(\mathbf{G}_{t}\right),
\end{gathered}
$$

where $\omega_{\mathbf{k}_{,} n}^{(\mathrm{TM})}$ and $\omega_{\mathbf{k}_{t}, n}^{(\mathrm{TE})}$ denote the eigenfrequencies of $E_{z, \mathbf{k}_{t},}\left(\mathbf{G}_{t}\right)$ and $H_{z, \mathbf{k}^{\prime}}\left(\mathbf{G}_{t}\right)$ respectively.

Equations 2.74 and 2.75 are the starting relations for the diagonalization method. In 2D, the computation is less expensive than in 3D. Figure 2.13 shows the results of the diagonalization method used in a computation tool designed by Professor Gauthier and a 
QL method implemented by the author. Figure 2.13(a) is the bandstructure obtained for a square array of high dielectric rods $\left(\varepsilon_{r}=12\right)$ in a low dielectric background $\left(\varepsilon_{r}=1\right)$, while in figure 2.13(b) is the bandstructure obtained for a hexagonal array of low dielectric rods $\left(\varepsilon_{r}=1\right)$ in a high dielectric background $\left(\mathcal{E}_{r}=12\right)$. The results showed complete agreement with results in the literature.



Figure 2.13. Planewave diagonalization results for bandstructure of (a) square array of high dielectric rods in low dielectric background and (b) hexagon array of low dielectric holes in a high dielectric background.

A PhQ structure was then analyzed with this technique. Due to the lack of translational symmetry in PhQs, concepts such as reciprocal lattices and Brillouin zones are not applicable to PhQs. Regardless, one can approximate a $\mathrm{PhQ}$ by enforcing a large 
primitive cell on the structure. Figure 2.14 shows two primitive cells: (a) the primitive cell of a hexagonal structure and (b) the "approximate" primitive cell of a 12-fold quasicrystal. The PhQ bandstructure for the $\mathrm{PhQ}$ structure in figure 2.11 is shown in figure 2.15 .

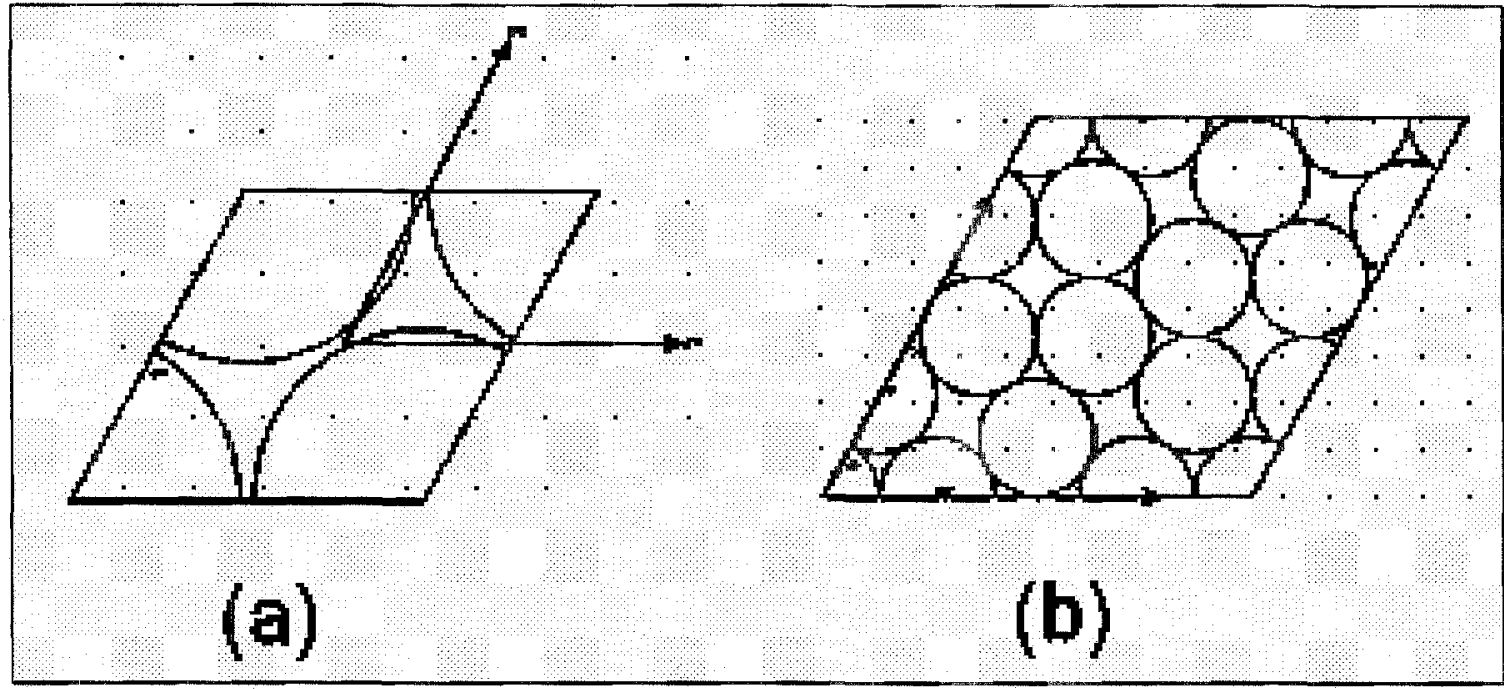

Figure 2.14. Primitive cells for planewave analysis. (a) hexagonal array primitive cell (b) 12-fold quasicrystal 'primitive' cell

The majority of this bandstructure resembles free space, implying that if one could define a Brillouin zone, it would be almost spherical. At the same time there are also PBGs. This prompted some researchers in the field to assert that lower dielectric contrasts are required when using PhQ lattices [21]. Instead of using a silicon-air type structure, one can make PBG materials with glass-air structures.

Bandstructures and diffraction patterns are related. One can tell that if the bandstructure of the PhQ is complicated, then the PhQ's diffraction pattern will also be complicated. If one makes a $\mathrm{PhQ}$ structure or one takes a naturally occurring quasicrystal and finds the diffraction patterns, one finds that their reciprocal lattice space is much more complicated than those of $\mathrm{PhC}$ or naturally occurring crystals. The diffraction patterns of PhCs have strong areas of constructive and destructive interference while the diffraction pattern of $\mathrm{PhQs}$ also have strong areas of constructive and destructive 
interference in addition to weaker areas of constructive and destructive interference. Four structures and their associated diffraction patterns are shown in figure 2.16. Top row images are of the structures themselves, which are (from left to right), naturally occurring AlCuFe quasicrystals, 10-fold $\mathrm{PhQ}$ fabricated in silicon, 12-fold $\mathrm{PhQ}$ fabricated in silicon, and $\mathrm{PhC}$ hexagonal lattice fabricated in photoresist. The bottom row shows their associated diffraction patterns. Structures (b), (c), and (d) were all fabricated by Professor Gauthier's group at Carleton University. One will notice that quasicrystal diffraction patterns are much more complicated than regular $\mathrm{PhC}$ patterns. The diffraction patterns for the PhQs and PhC were photographed in a way shown in figure 2.17. The sample was held in place near a white wall, where laser light was then directed at the sample, and the diffracted light was then projected on to the white wall. An image with a camera was then taken.

In conclusion, the planewave approach can only give a rough picture of the bandstructure of PhQs. The important feature of the planewave analysis is to determine whether a PhQ has a band gap. If it does, then a defect may be introduced in such a way as to admit an application such as waveguiding and cavity resonances for add/drop ports. This is the same reasoning used with when dealing with PhCs. However, understanding how the band structure arises and why band gaps occur cannot be directly taken from the planewave approach. Another analytical technique that is computationally effective and physically insightful that has been used in the literature is similar to the Kohn-KorringaRostoker (KKR) approach. It uses a Green's function approach to solve for the bandstructure. 


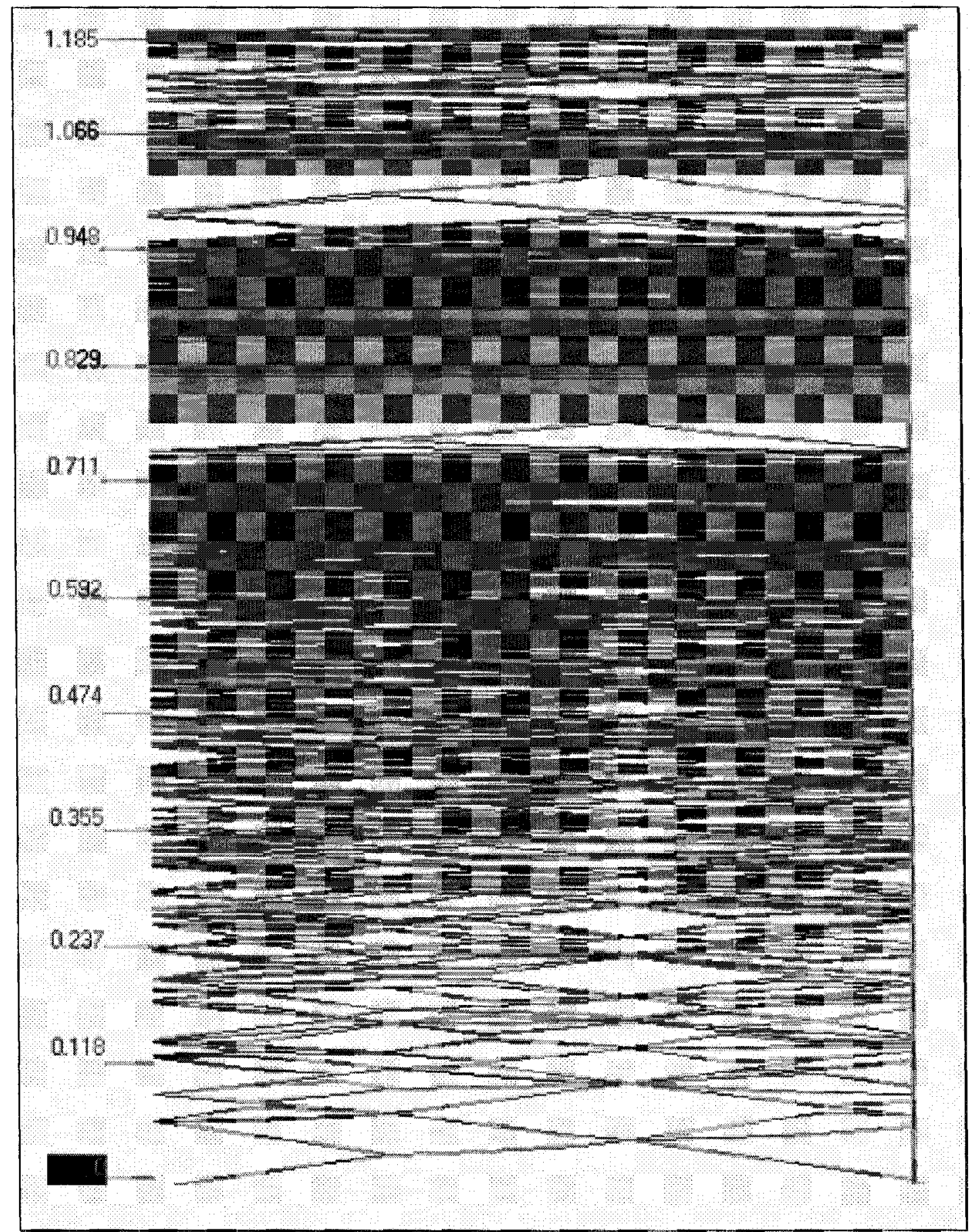

Figure 2.15. Example of $\mathrm{PhQ}$ bandstructure calculated from the planewave expansion. 


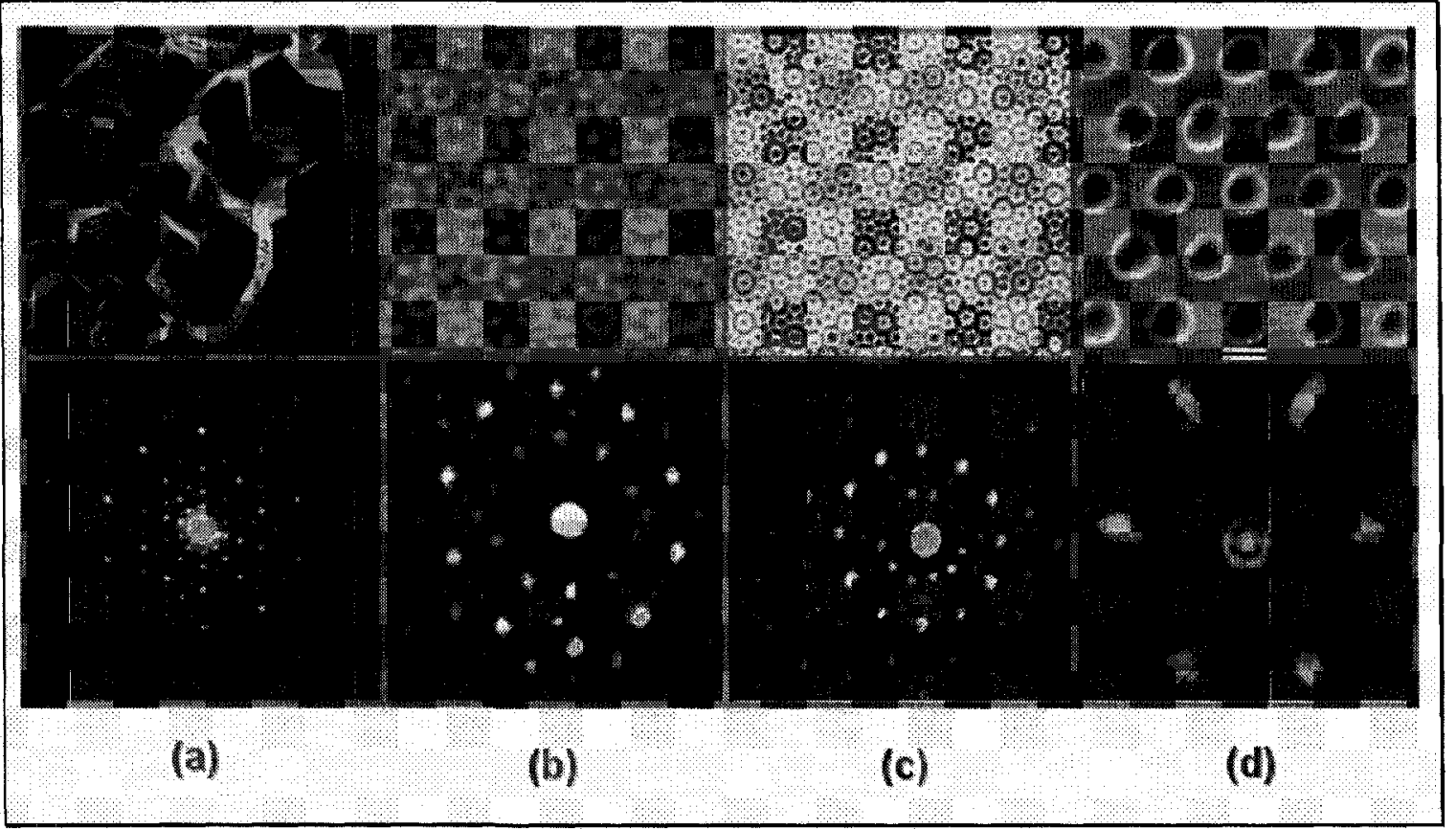

Figure 2.16. Top row images are of real structures and bottom row images are diffraction patterns. (a) Naturally occurring AlCuFe quasicrystal, (b) 10-fold PhQ fabricated in silicon, (c) 12-fold $\mathrm{PhQs}$ fabricated in silicon and (d) hexagon $\mathrm{PhC}$ fabricated in photoresist.

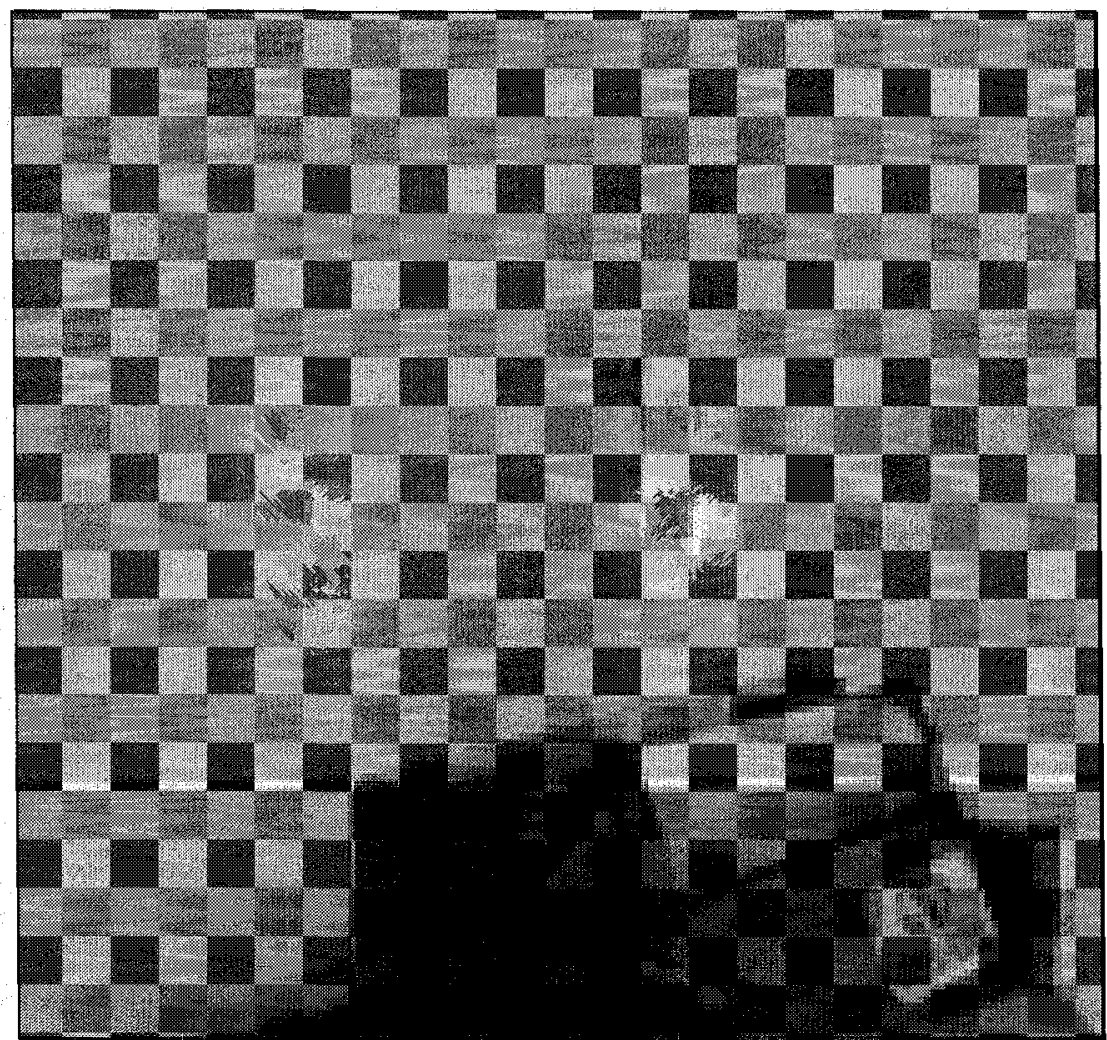

Figure 2.17. Obtaining the diffraction pattern on PhQs and PhC patterns. 


\subsubsection{Green's Function Approach}

The diagonalization planewave analysis and variational problem planewave analysis techniques are geared towards translationally symmetric structures such as PhCs. They can be also be applied to PhQs in an approximate way, as discussed earlier. However, the only feature that can be determined is if the PhQ has a band gap, and the range of this band gap. The planewave analysis does not explain the true bandstructure nor does it provide a physical insight into how and why these bandstructures are formed. Before having to resort to purely numerical techniques, one analytical technique can be used to gain insight into the bandstructure of PhQs. Due to the rotational symmetry PhQs have, one can exploit a solid-state physics technique known as the Kohn-Korringa-Rostoker (KKR) method that uses an integral approach in solving for the bandstructure based upon spherical harmonics. Because it is an integral approach, the kernel of the integral is a Green's function. More information on Green's functions can be found in the Appendix

II. By determining the Green's function of the partial differential equations and boundary conditions defined by the structure in question, one can find the density of states (DOS) which is another way of expressing the band structure of the PhQ.

If one takes the 2D modified electric field operator,

$$
L_{Q}^{2 D}=-\frac{1}{\sqrt{\varepsilon_{r}(\mathbf{r})}} \nabla^{2}, \frac{1}{\sqrt{\varepsilon_{r}(\mathbf{r})}},
$$

and modified electric field,

$$
Q_{z}(\mathbf{r})=\sqrt{\varepsilon_{r}(\mathbf{r})} E_{z}(\mathbf{r}),
$$

the eigenvalue equation becomes, 


$$
L_{Q}^{2 n} Q_{z}(\mathbf{r})=\frac{\omega^{2}}{c^{2}} Q_{\theta}(\mathbf{r}) \Rightarrow\left[\varepsilon_{r}(\mathbf{r}) \frac{\omega^{2}}{c^{2}}+\nabla_{t}^{2}\right] E_{z}(\mathbf{r})=0
$$

The Green's function is then defined in the usual way as,

$$
\left[\varepsilon_{r}(\mathbf{r}) \frac{\omega^{2}}{c^{2}}+\nabla_{t}^{2}\right] G(\mathbf{r}, \omega)=\delta\left(\mathbf{r}-\mathbf{r}_{0}\right)
$$

In Appendix II, the local density of states (LDOS) is derived from the knowledge of the Green's function as,

$$
\rho\left(\mathbf{r}_{0}, \omega\right)=\operatorname{Im}\{\operatorname{Tr}[G(\omega)]\}
$$

where $\mathbf{r}_{0}$ is an arbitrary location in the structure, Im denotes the procedure of taking the imaginary part of a complex number, and $\operatorname{Tr}$ denotes the trace of a matrix. In this case, the trace of the function implies that the spatial coordinate is taken at some known point.

For a generic dielectric structure, equation 2.79 is difficult if not impossible to solve. However, if the dielectric structure is made of rods, such as what is shown in figure 2.12, then the Green's function can be built from the multipole expansion about each rod. As an example of this method, take the structure shown in figure 2.18. 


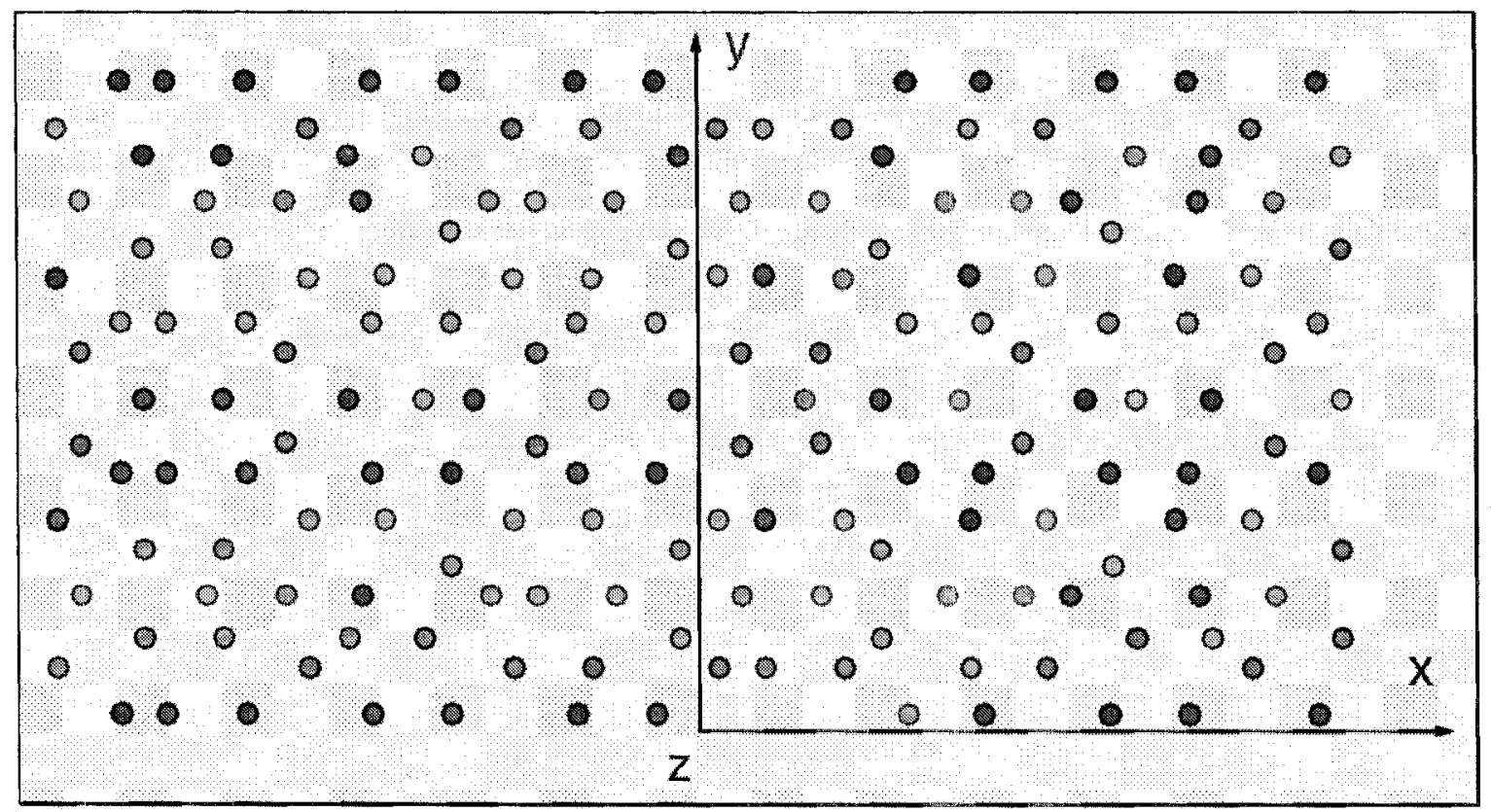

Figure 2.18. Ten-fold symmetric Penrose tiling. High dielectric rods placed at vertices of the tilings. [37]

When the multipole expansion for each rod is taken and used to find the Green's function, the LDOS of this structure is shown in figure 2.19.

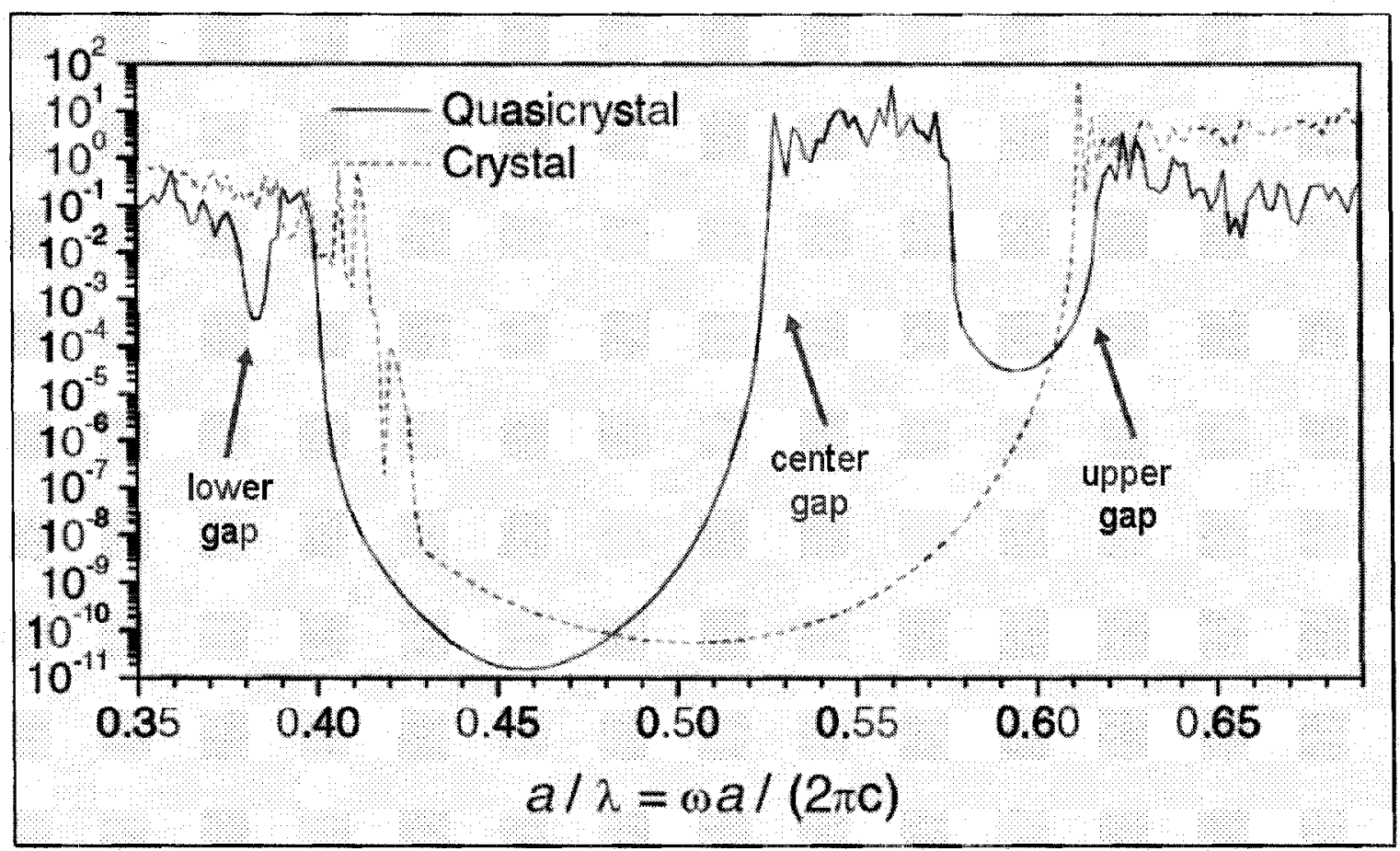

Figure 2.19. LDOS for PhQ structure in figure 2.18 (solid line) and square lattice of dielectric rods $\mathrm{PhC}$. [37]

Figure 2.19 shows large dips in the LDOS of the structures. These dips correspond to the band gaps in the bandstructures of the PhQ and PhC. The most 
interesting result of this work is when the analysis was made for a shrinking number of rods. The results are shown in figure 2.20.

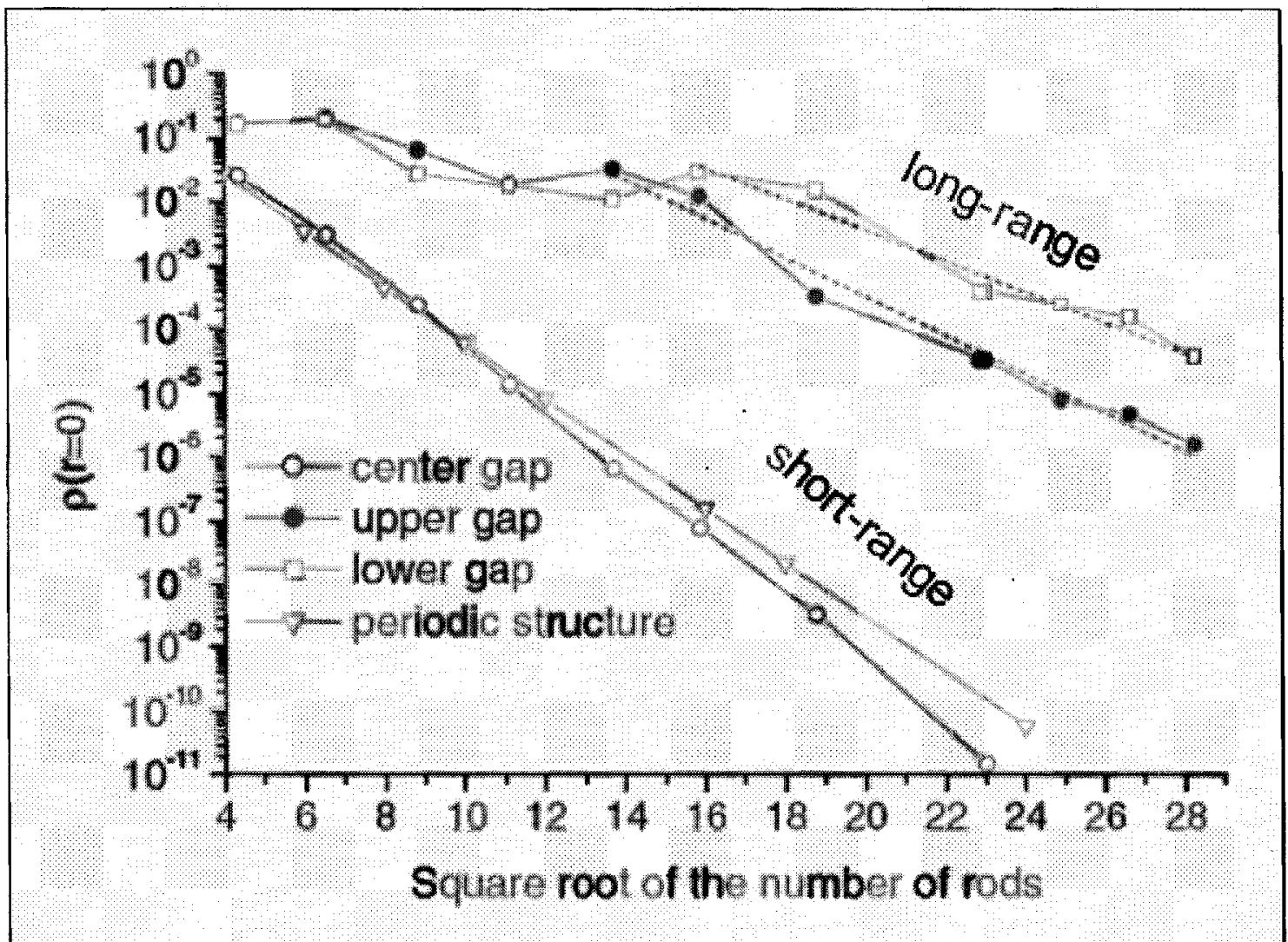

Figure 2.20. LDOS graph as a function of rod count. [37]

What figure 2.20 indicates is that some band gaps are short-range dependent while other band gaps are long-range dependent. It was originally thought that the bandstructure was due to the long-range order in the PhQ. However, these results show that there are short-range affects that are responsible for the bandstructure. This is counterintuitive because with light being a non-interacting bosonic field, tight-binding theories that explain bandstructure formation in amorphous electronic structures cannot be applied for photon fields. One needs to determine from where this short-range dependence comes from. 


\subsubsection{The Need for a Numerical Approach}

The planewave approximation of $\mathrm{PhQs}$ is effective in giving an overall picture of where the PBGs may be within the bandstructure. This is useful for one who would then introduce a defect into the physical structure and use the defect modes as waveguides or add/drop ports for functional devices. However, due to the lack of translational periodicity, the planewave technique is inadequate for describing PhQs in a rigorous manner. A rotational view of the planewave technique may exist, but for now, concepts such as a rotational reciprocal space and rotational Brillouin zones are ill-defined.

The Green's function approach is closer to being a more complete theory for PhQs. However, the actual Green's function is constructed from a moment method technique used for circular rods. Regardless, some very important results have occurred because of this. For example, the PBGs in PhQ bandstructures have been shown to have both short and long-range dependences. This feature would not have been discovered if one only used the planewave technique. Even though the Green's function approach adds more understanding, the scatterer shapes need to be circular. If the scatterer shapes become non-circular, then the Green's function becomes very difficult to determine. One is then not able to explore the dielectric form factor (DFF) and its effect on the PhQ bandstructure or the dynamics that occurs within it This is why the next chapter is dedicated to a purely numerical study of PhQs. 


\section{Numerical Analysis}

Current $\mathrm{PhC}$ and PhQ theory focuses on how the lattice generates the bandstructures, while the scatterer form factors are chosen to be of a conventional shape for ease of calculation and fabrication. As of yet, no work has been done on how the scatterer form factor in PhQs effects their bandstructures or the modes that occur within them. It is important to note that the shapes should not be arbitrary, since that will result in a disordered structure. One of the points of originality in this thesis is to study PhQs with unconventional scatterer form factors that are not arbitrary but are correlated to the symmetry of the quasicrystal lattice.

In this chapter, the author deals with describing how such PhQs can be designed and it studies their optical properties by simulating PhQs using two purely numerical techniques: finite-difference time-domain (FDTD) and finite-element method (FEM). The scatterer form factor will be renamed the dielectric form factor (DFF) since one is dealing with light.

The first few sections of this chapter describe an innovative technique known as dual-beam holographic lithography (DBHL), used for generating PhQs with correlated, unconventional DFFs. The DBHL technique is primarily used in this chapter as a method to computationally generate the patterns for simulation. However, it can be used to physically generate such structures. Refer to [34] for more on this technique.

The next few sections deal with an innovative FDTD technique that utilizes a movable window computational domain (MWCD) for obtaining the bandstructures of these PhQs. Within the literature, PhQs with defect sites were found to be more 
wavelength-selective than defect containing PhCs. As well, this means that they would be good candidates for components in DWDM systems because they would provide better coupling efficiencies. However, the immediate geometry around the defect will dictate the wavelength selected. Hence, in order to add/drop a range of wavelengths, the PhQ geometry must be graded. This analysis will result in something unexpected: localized modes with no defects in the PhQ pattern. Similar results were found in the literature for conventional DFFs $[8,9]$.

The rest of the chapter is dedicated to studying these localized modes. The FEM approach is used because it allows an easier implementation of an eigenvalue analysis. It will be shown that these localized modes are similar to angular momentum modes of the electron wavefunction around a central potential. The orbital angular momentum number is equal to the rotational fold number of the PhQ. It will be shown that all the angular momentum modes of a 12-fold PhQ can be found, which violates the predications of group theory. In addition, these first and second modes of the system are interchanged in energy, exactly like what occurs within atomic systems. This interchange or shift is known as the Lamb shift, and results from interactions with the quantum mechanical vacuum. The reason for this occurring in the PhQs has to do with the DFF and with minimal energy configurations. Minimal energy configurations arise from the variational principle. Since the variational principle is a mathematical minimizing function, it is independent of the physics [46]. The QED vacuum is not disguisable from a polarizable dielectric [47] and, hence, energy corrections in both optics and QED due to seen to have the same effect overall on the physics (i.e. lower the energy of one mode below that of another). 


\subsection{Photonic Quasicrystals Pattern Generation}

\subsubsection{Dual Beam Holographic Lithography Technique}

The DBHL technique is composed of two coherent laser beams interfering at some angle between them in a photosensitive medium. This results in plane interference fringes yielding planewaves with a well-defined normal (i.e. wavevector). An experimental setup of a system is shown in figure 3.1 .

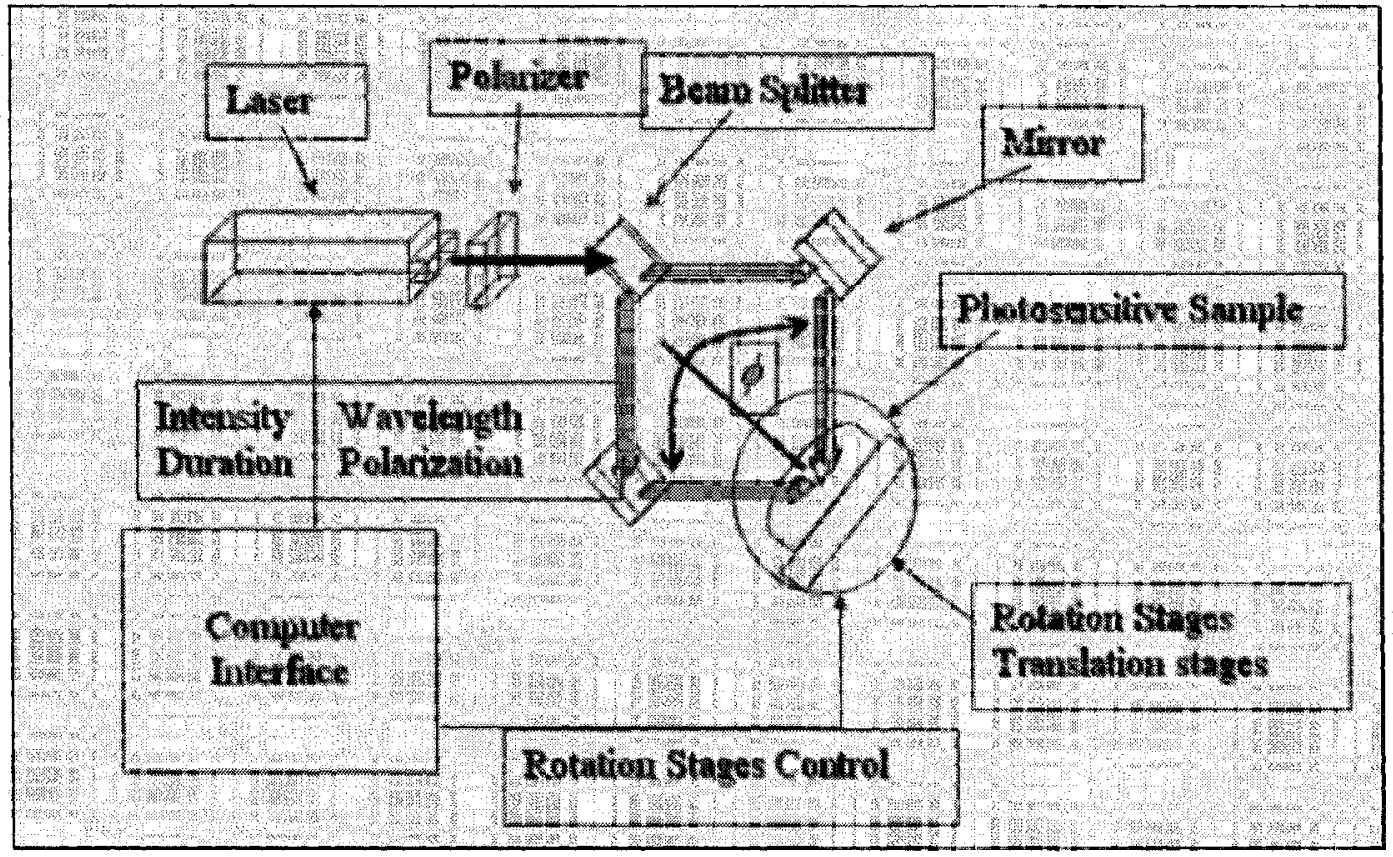

Figure 3.1. Experiment setup for DBHL.

The laser is polarized immediately so that polarization effects do not distort the interference pattern. The beam is then split by a beamsplitter into two orthogonal beams diverging from each other at some given angle. They are then redirected by mirrors to converge and interfere in a photosensitive sample. The angle they interfere at which is denoted by $\phi$, controls the spacing of the planes generated from the dual beam interference. To obtain the smallest spacing between planes, the beams need to be at $180^{\circ}$ to each other. 
The governing equation of the DBHL system is,

$$
I=E_{1}^{2}+E_{2}^{2}+E_{1} E_{2} \cos \left(\left[\mathbf{k}_{1}-\mathbf{k}_{2}\right] \cdot \mathbf{r}+\varphi_{1}-\varphi_{2}\right) \cos \theta_{12}
$$

where $I$ is the resultant intensity of the interfering beams, $E_{1}$ and $E_{2}$ are the electric field amplitudes, $\mathbf{k}_{1}$ and $\mathbf{k}_{2}$ are the associated wavevectors of the beams, $\varphi_{1}$ and $\varphi_{2}$ are the initial phase factors of beams and $\theta_{12}$ azimuthal angle between them. Since they are in the same plane, then $\theta_{12}=0$, which removes fringing.

The $\left[\mathbf{k}_{1}-\mathbf{k}_{2}\right]$ relation in equation 3.1 is similar to the Laue condition discussed in Chapter 2. In this particular case, $\mathbf{k}_{1}$ and $\mathbf{k}_{2}$ are incoming wavevectors. The wavevector of the generated planes is then similar to the reciprocal lattice vector, $\mathbf{K}$. The DBHL process is essentially a reverse crystallography method. Figure 3.2 shows this process with the related plane equation and the relation for plane separation.

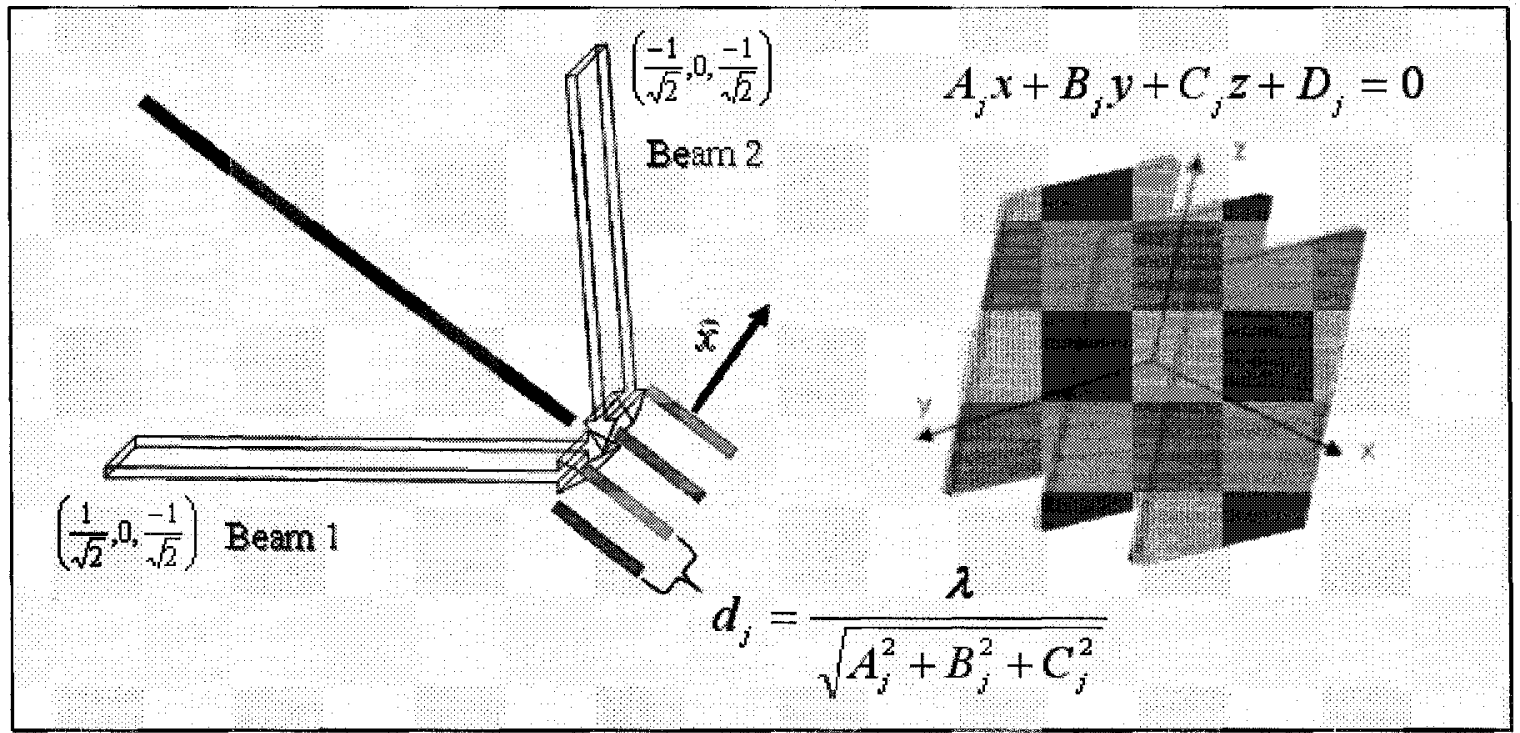

Figure 3.2. Planewave generation via the DBHL process. 


\subsubsection{Crystal and Quasicrystal Lattice Generation}

Now that planewaves with a definite wavevector can be generated in a photosensitive medium, other planewave families with different wavevectors can be generated by simply rotating the stage on which the photosensitive media sits. The coefficients of the plane equation can then be directly related to the Euler angles of the rotation stage, as shown in figure 3.3.

$$
\left[\begin{array}{l}
A \\
B \\
C
\end{array}\right]=\left[\begin{array}{c}
-\cos (\varphi) \cos (\phi)+\sin (\varphi) \cos (\theta) \sin (\phi) \\
\sin (\varphi) \cos (\phi)+\cos (\varphi) \cos (\theta) \cos (\phi) \\
-\sin (\theta) \sin (\phi)
\end{array}\right]
$$

Solve for rotation angles

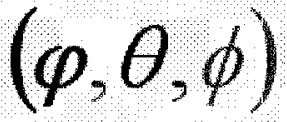

Sample orientation angles

Figure 3.3. Relationship between Euler angles of stage and plane equation coefficients.

Different lattices can then be created in the resist by interfering different plane families. This is similar to constructing the reciprocal lattice of some direct lattice, such as a square or triangular lattice, as shown in figure 3.4. Figure 3.4(1a) and figure 3.4(1b) are plane intersection schematics. If the cumulative intensity of these regions are enough to fully expose the photosensitive medium, then after development, structures resembling figure 3.4(2a) and figure 3.4(2b) result, having lattice types of figure 3.4(3a) and figure $3.4(3 b)$.

One can control the crystal basis by equating the exposure threshold of the medium to a relative intensity ratio that is a percentage of the overall full intensity of the total number of the exposures per rotation. This ratio is known as the dielectric fill factor of the finished structure. As an example, consider the simulated generation of a triangular lattice as shown in figure 3.5. Each exposure leading to a planewave family contributes four units of intensity to the photosensitive medium. Three exposures with a 
relative $60^{\circ}$ angle between them yields 12 units of intensity delivered to the photosensitive medium. The three exposure threshold ratios shown in figure 3.5 are set to the actual exposure dose of the physical medium. This results in three different basis sizes. Figure 3.6 shows the result of a real implementation of the DBHL in a real photoresist medium. The resist used here is called $\$ 1811$ made by the Shipley® Corporation.

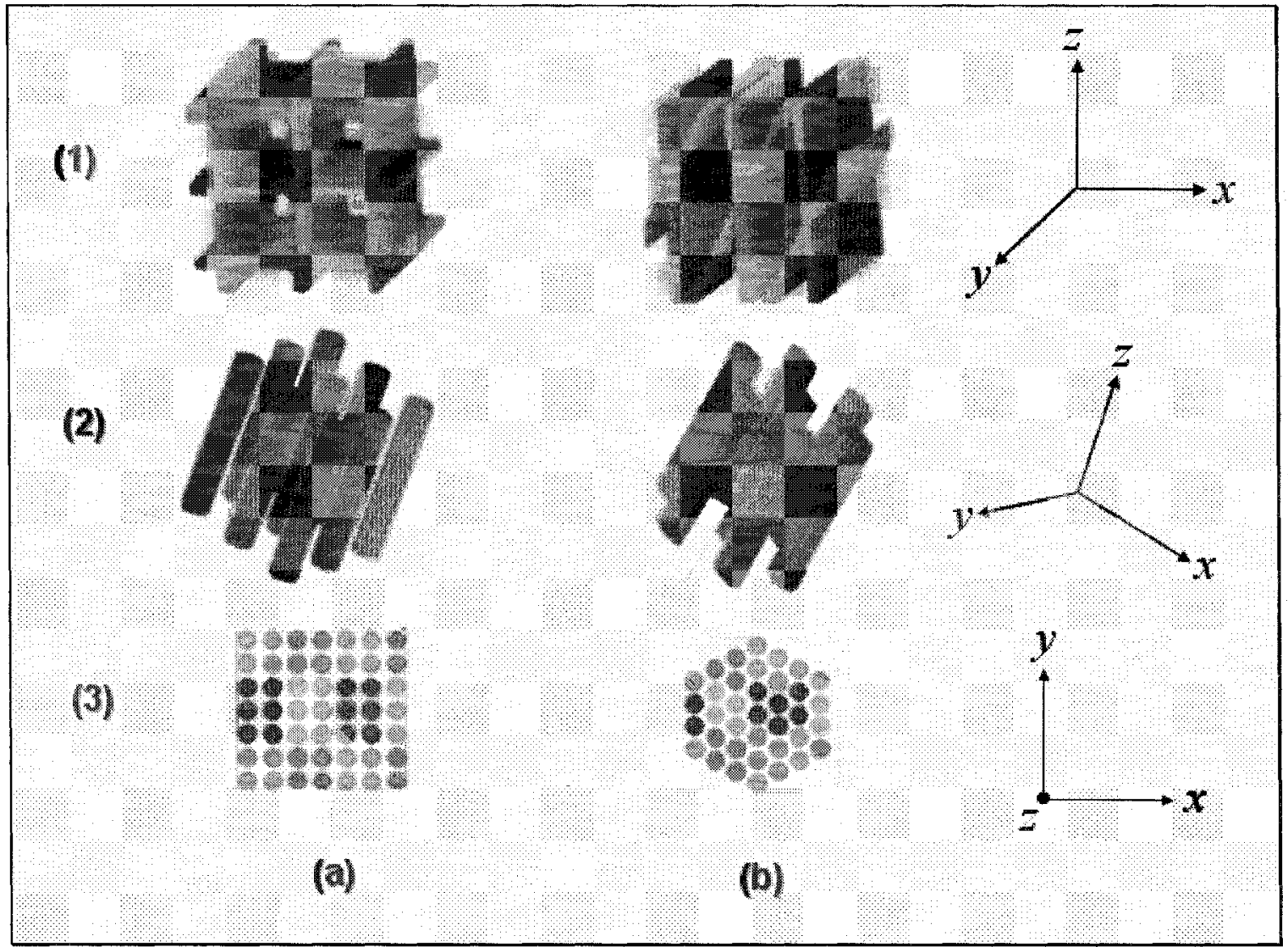

Figure 3.4.. Interfering plane families (1) lead to crystal structures (2) with different lattice types (3). (a) Square lattice and (b) triangular lattice. Associated direction axes shown at far right.

Quasicrystal patterns can be generatcd by sclecting a rotation between cxposurcs that do not lead to translationally periodic patterns. Figure 3.7 shows (a) a planewave schematic view where six families of planewaves are interfering at $30^{\circ}$ and (b) the resultant structure after the resist conversion dose has been set to a specific threshold 
ratio. In this case, since there are six exposures, the maximum 24 units of intensity are delivered to the photosensitive medium. This generates a 12-fold quasicrystal pattern. Figure 3.8 shows a variety of 12 -fold quasicrystal patterns for threshold levels set at 7, 8 , $10,12,14,15,16,18$ and 20 out of 24 . It shows the variation in feature sizes obtained at these different levels of exposure.

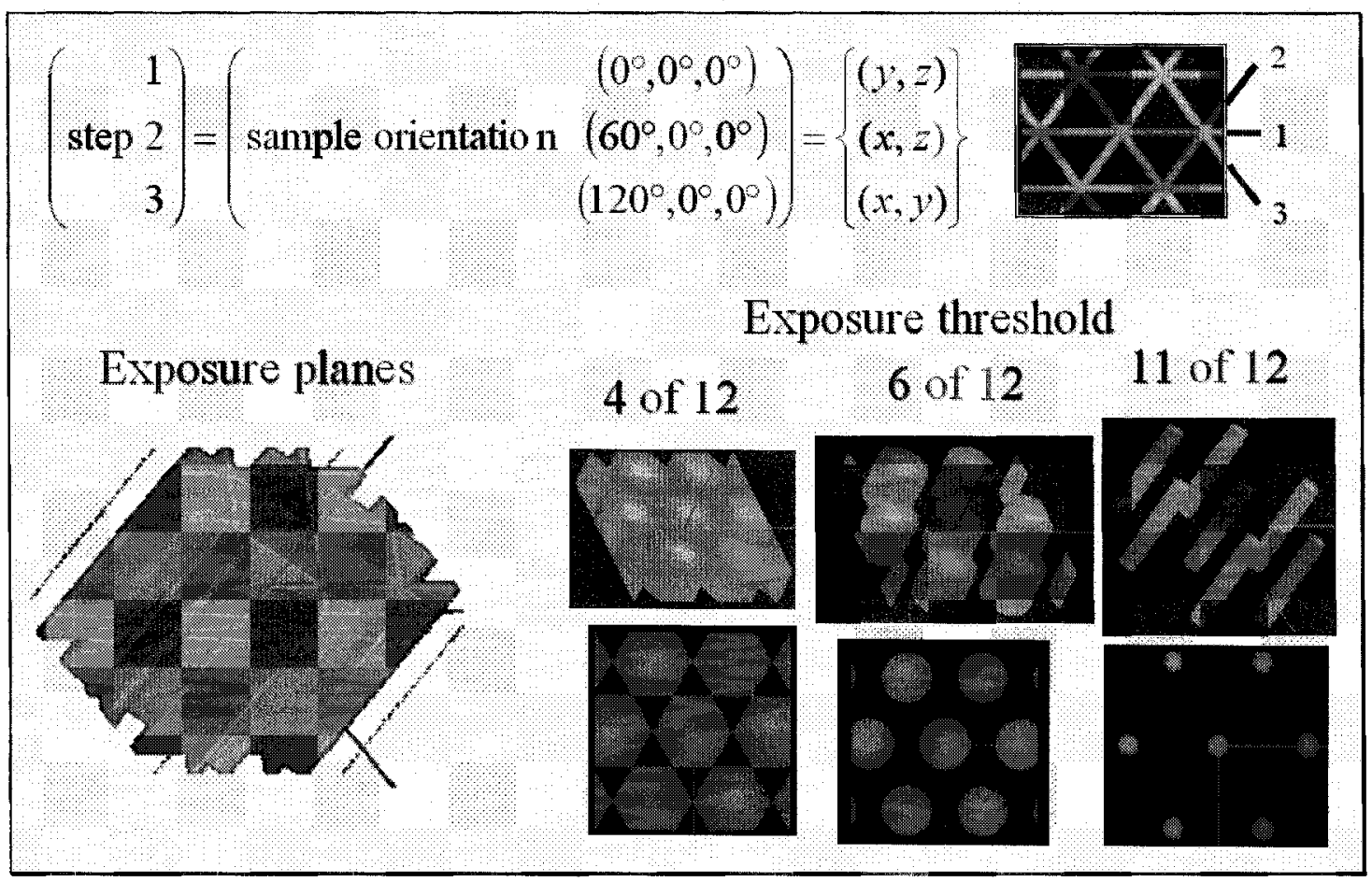

Figure 3.5. Planewave schematic view of creating periodic structures and their relative threshold values in resist. 


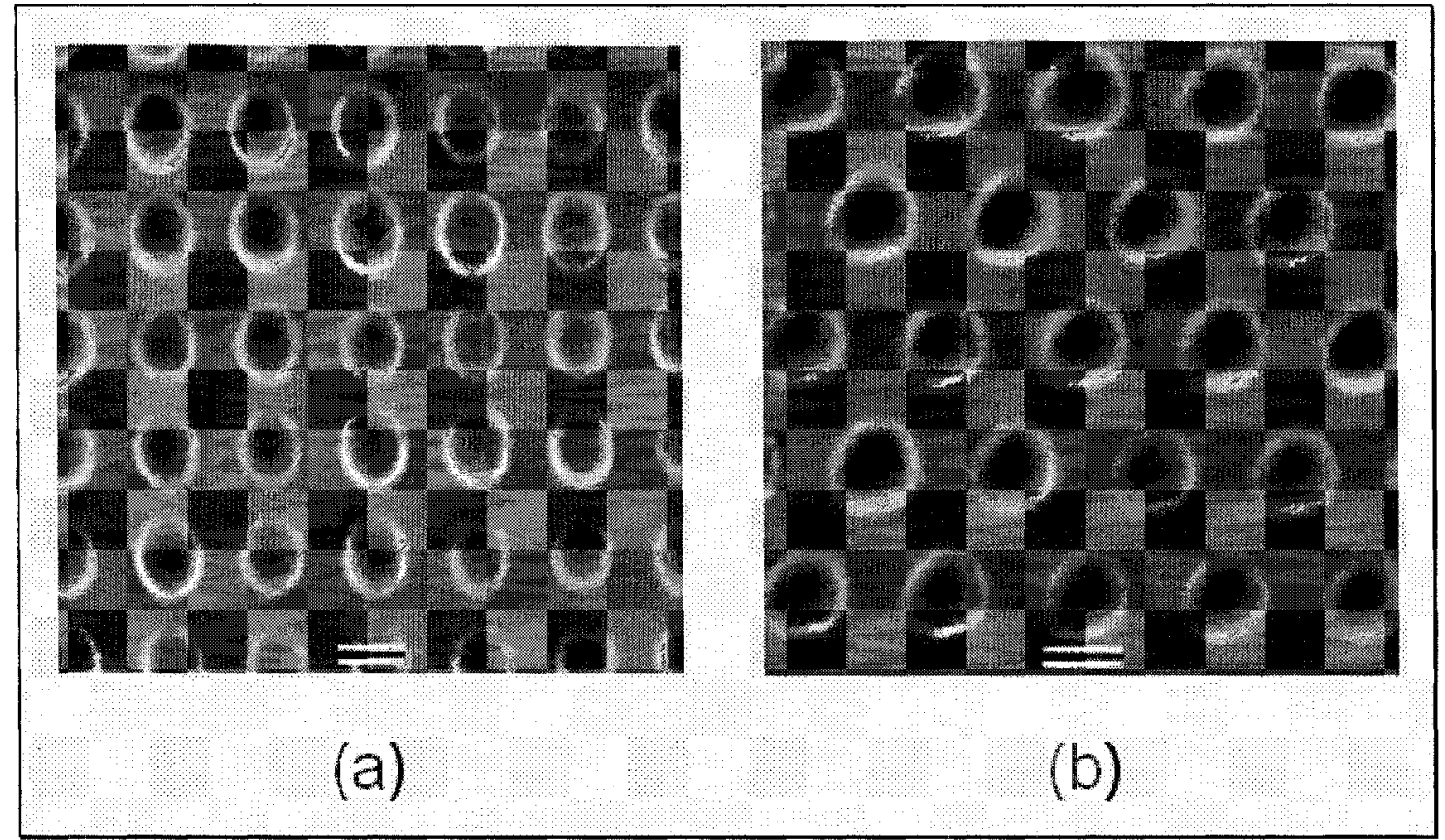

Figure 3.6. Physical structures of dual beam holographic exposure method. Bar length in both images is one micron.

Computationally, this method was simulated by two codes: a custom tool by Professor Robert C. Gauthier, and one created by the commercial software, Mathematica®. Figure 3.7 was created from the software designed by Professor Gauthier and figure 3.9 is a snapshot of the results of Mathematica ${ }^{\circledR}$ code producing a $\mathrm{PhQ}$ structure. Once the structures can be constructed via these simulation methods, they can then be exported to other commercial solvers or custom software for numerical analysis. A useful and effective tool to commence analysis of complicated structures is the FDTD method. 


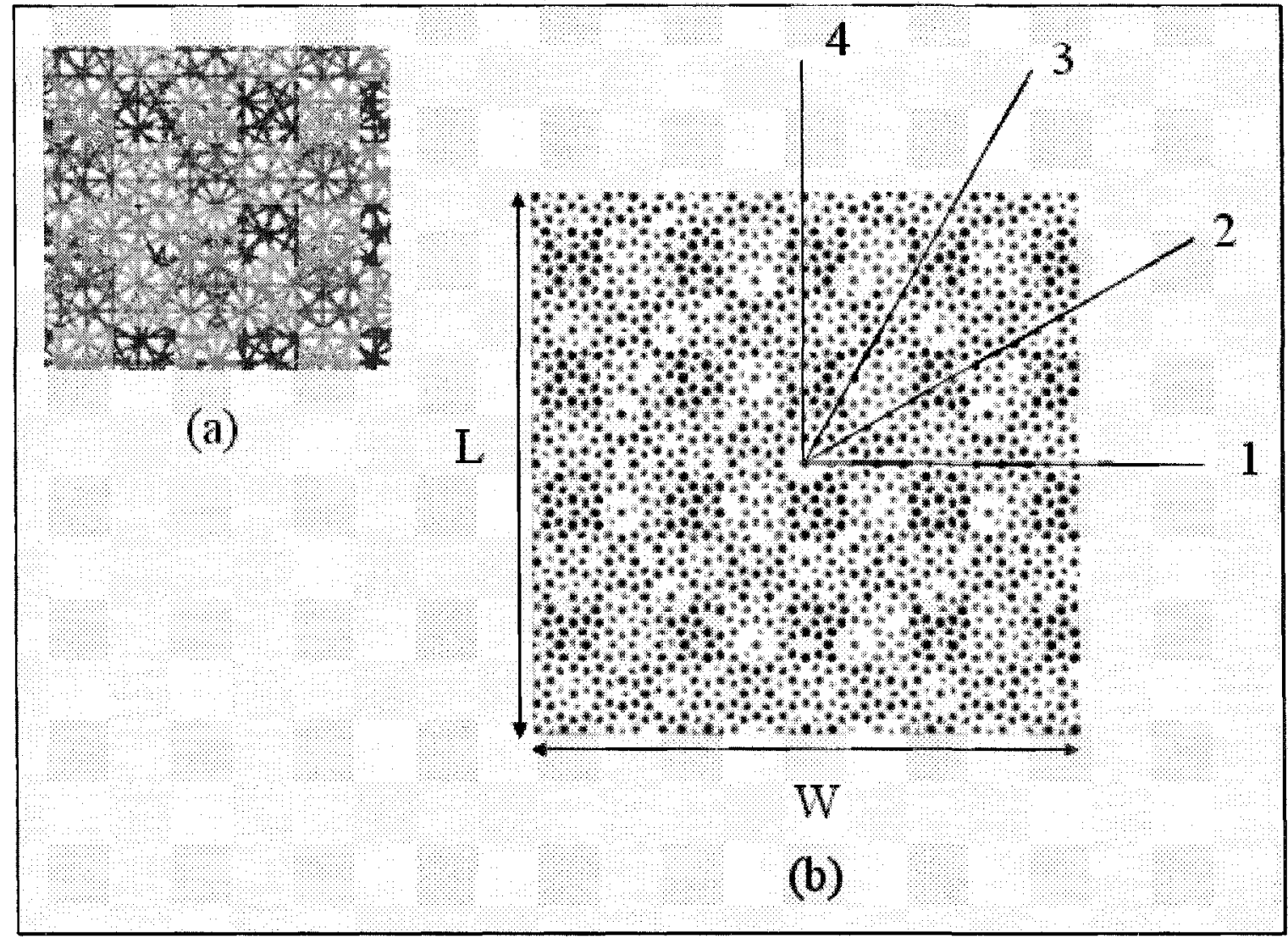

Figure 3.7. Creating quasicrystal structures. (a) planewave schematic and (b) some set threshold ratio to generate physical structure. 


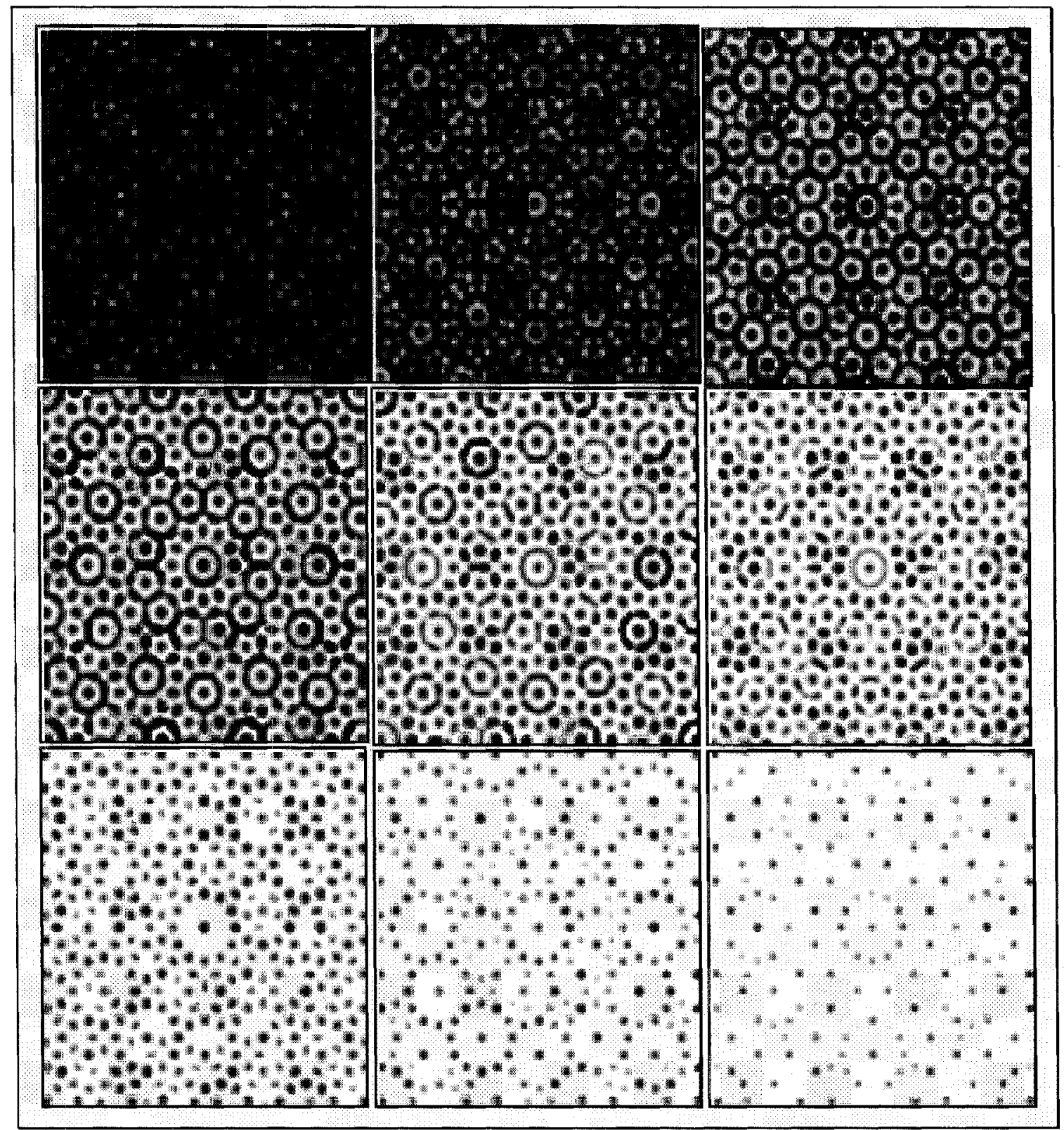

Figure 3.8. PhQ patterns at different exposure thresholds yielding the different fill factors. Starting from top left, reading across, then down by rows, the thresholds of 7,8 , $10,12,14,15,16,18$ and 20 , out of a maximum of 24 


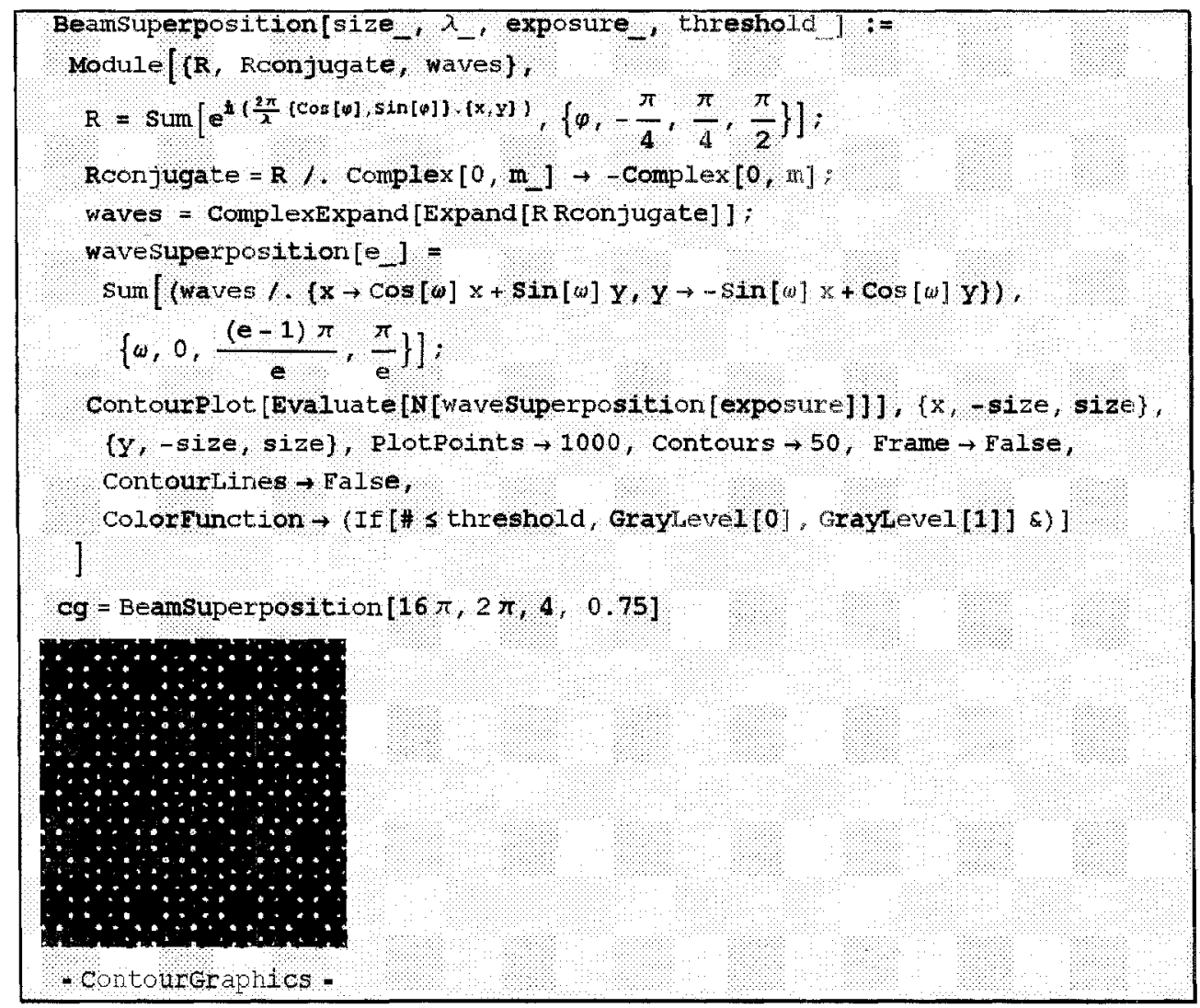

Figure 3.9. Screen capture of Mathematica software generating quasicrystal patterns.

\subsection{Finite-Difference Time-Domain Technique}

\subsubsection{Theoretical Basis}

When structures have complicated geometries, one of the best methods used in computational analysis for investigation is the finite-difference-time-domain (FDTD) discretizations of Maxwell's equations. Since the work in this thesis deals with 2D structures, only three of six fields are required to be solved for at one time: the TM fields $\left(E_{z}, H_{x}, H_{y}\right)$ or the TE fields $\left(H_{z}, E_{x}, E_{y}\right)$. From these two choices, this work deals primarily with the TM fields. The disconnected topology of PhQ rods are more conducive to forming a bandstructure with band gaps for TM fields than for TE fields. The reason for this is that the scalar $D_{z}$ field of the TM modes can be localized within 
the rods, but the continuous field lines of the TE modes are compelled to penetrate the air regions to connect neighboring rods. Therefore, consecutive TE modes cannot exhibit markedly different fractions of electric energy located in the high- $\varepsilon$ regions.

Taking equations $2.5,2.12$ and 2.13 and selecting the TM portion, one has

$$
\begin{gathered}
\frac{\partial D_{z}}{\partial t}=\frac{1}{\sqrt{\varepsilon_{0} \mu_{0}}}\left(\frac{\partial H_{y}}{\partial x}-\frac{\partial H_{x}}{\partial y}\right) \\
\frac{\partial H_{x}}{\partial t}=-\frac{1}{\sqrt{\varepsilon_{0} \mu_{0}}} \frac{\partial E_{z}}{\partial y} \\
\frac{\partial H_{y}}{\partial t}=\frac{1}{\sqrt{\varepsilon_{0} \mu_{0}}} \frac{\partial E_{z}}{\partial x}
\end{gathered}
$$

Putting these equations into a finite-differencing scheme, as shown in figure 3.10, gives

$$
\begin{aligned}
& \frac{D_{z}^{n+1 / 2}(i, j)-D_{z}^{n-1 / 2}(i, j)}{\Delta t}=\frac{1}{\sqrt{\varepsilon_{0} \mu_{0}}}\left(\frac{H_{y}^{n}(i+1 / 2, j)-H_{y}^{n}(i-1 / 2, j)}{\Delta x}\right) \\
& -\frac{1}{\sqrt{\varepsilon_{0} \mu_{0}}}\left(\frac{H_{x}^{n}(i, j+1 / 2)-H_{x}^{n}(i, j-1 / 2)}{\Delta y}\right) \\
& \frac{H_{x}^{n+1}(i, j+1 / 2)-H_{x}^{n}(i, j+1 / 2)}{\Delta t}=-\frac{1}{\sqrt{\varepsilon_{0} \mu_{0}}} \frac{E_{z}^{n+1 / 2}(i, j+1)-E_{z}^{n+1 / 2}(i, j)}{\Delta y}, \\
& \frac{H_{y}^{n+1}(i+1 / 2, j)-H_{y}^{n}(i+1 / 2, j)}{\Delta t}=\frac{1}{\sqrt{\varepsilon_{0} \mu_{0}}} \frac{E_{z}^{n+1 / 2}(i+1, j)-E_{z}^{n+1 / 2}(i, j)}{\Delta x}
\end{aligned}
$$




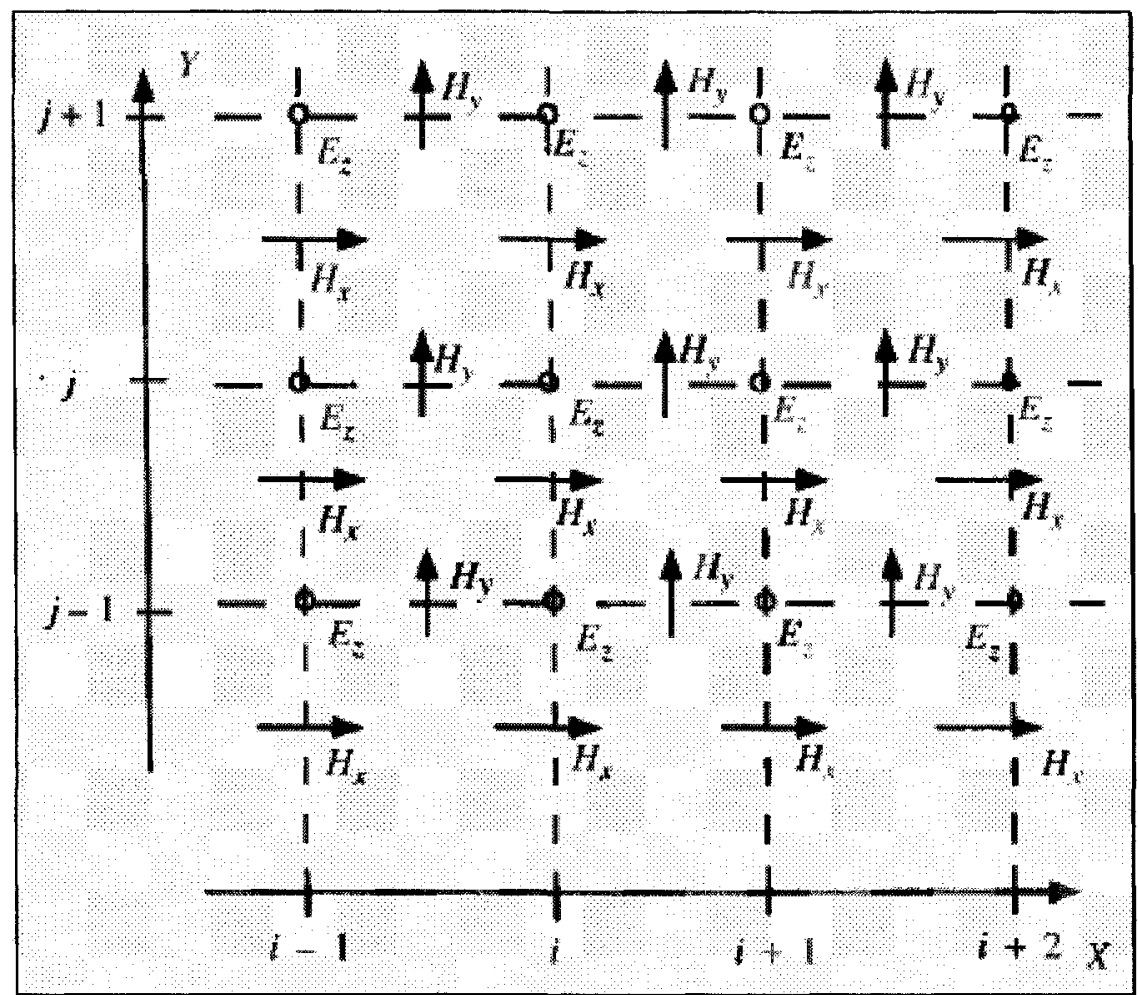

Figure 3.10. Finite-difference scheme and field definitions for the discrete 2D calculation lattice [48].

Time is specified by the superscript $n$, which means $t=\Delta t \cdot n$, where $\Delta t$ is a carefully selected time step that must incorporate the fact that one is dealing with electromagnetic waves that cannot travel faster than light. The time step condition, also known as the Courant condition, must be

$$
\Delta t \leq \frac{\Delta x}{\sqrt{\operatorname{dim}} \cdot c_{0}}
$$

where $\Delta x$ is the space step selected for the problem, dim is equal to the dimension of the space and $c_{0}$ is the speed of light in a vacuum. A rule of thumb is to insure that $c_{0} \Delta t / \Delta x \leq 0.5$. Space steps are specified by the $(i, j)$ pair, where $x=\Delta x \cdot i$ and $y=\Delta y \cdot j$. It is common to have $\Delta x=\Delta y$. 
After the space and time stepping parameters have been determined, the order of field computations needs to be consistent with Maxwell's equations. This field stepping scheme is shown in figure 3.11. However, the above description is for the model space of a discretized structure. One needs to place a calculation boundary around this model space. The boundary cannot cause reflections because one would then obtain unphysical results since there is no boundary around the real structure to be modeled.

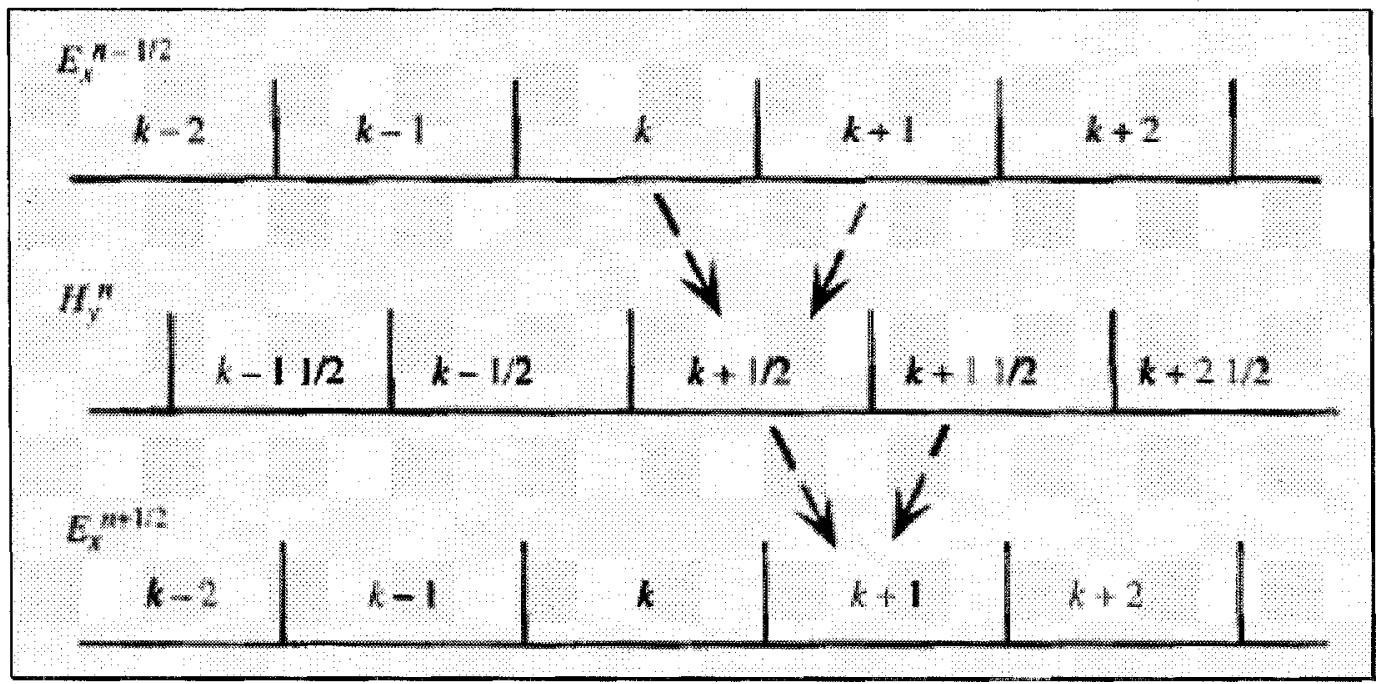

Figure 3.11. Stepping scheme for field computations. The $k$ term represents both $i$ and $j$ in equations 3.5 to 3.7 .

One effective way of dealing with this problem is to surround the model space with an artificial medium that causes no reflections and is so lossy that by the time the wave arrives at the calculation boundary it has become negligible. This artificial medium is known as a perfectly matched layer (PML). The PML must contribute to a zero reflection coefficient or close to a zero reflection coefficient after two passes through the PML. The reflection coefficient should be,

$$
\Gamma=\frac{\eta-\eta_{\mathrm{PML}}}{\eta+\eta_{\mathrm{PML}}}=0
$$


where $\eta$ is the intrinsic impedance of the model space, and $\eta_{\mathrm{PML}}$ is the intrinsic impedance of the PML. The intrinsic impedances then has the following form,

$$
\begin{gathered}
\eta=\sqrt{\frac{\mu}{\varepsilon}} \\
\eta_{\mathrm{PML}}=\sqrt{\frac{\mu_{\mathrm{PML}}\left(1+\sigma^{*} / j \omega \mu_{\mathrm{PML}}\right)}{\varepsilon_{\mathrm{PML}}\left(1+\sigma / j \omega \varepsilon_{\mathrm{PML}}\right)}}
\end{gathered}
$$

If one then sets $\varepsilon=\varepsilon_{\mathrm{PML}}$ and $\mu=\mu_{\mathrm{PML}}$ and enforces the condition:

$$
\frac{\sigma^{*}}{\mu}=\frac{\sigma}{\varepsilon} \rightarrow \sigma^{*}=\sigma \frac{\mu}{\varepsilon}=\sigma \eta^{2}
$$

then $\eta=\eta_{\mathrm{PML}}$, satisfying 3.9 . The wavenumber in the two media are also equal. This implies that the propagation constant in the PML becomes,

$$
\beta_{\mathrm{PML}}=\left(1+\frac{\sigma}{j \omega \varepsilon}\right) k=k-j \sigma \eta
$$

where $k$ is the wavenumber in the model space. This result shows that the fields in the PML will be exponentially decreasing by $\sigma \eta$.

\subsubsection{FDTD Analysis of Photonic Quasicrystals}

The FDTD technique is very effective in studying situations that have little or no analytic theory. This is why FDTD is a good first start in trying to characterize the electrodynamics of PhQs. If something interesting is found requiring a more detailed analysis, frequency domain methods can then be used to gain more information. This is the method followed here in this thesis. The FDTD technique was initially used to study the application of PhQs for DWDM applications. This analysis ended up showing that 
there was light localization without any of the structure containing physical defects from its original pattern, resulting in the concept of "defectless" defect modes. From this point, a frequency domain analysis (i.e. eigenvalue analysis implemented via the Finite Element Method) was done. This analysis showed that these "defectless" defect modes resembled those angular momentum modes of a central potential in quantum mechanics. Details of this analysis can be found in the next section.

The FDTD software used in this work was written by Professor Robert C. Gauthier using a Visual Basic compiler. This tool is similar to commercially available FDTD software but has a built-in PhQ pattern generator. The PhQ patterns are specified by indicating the optical properties of the substrate and fill material (air), the rotational order, and the threshold exposure level of the photosensitive material. The pattern is then discretized into a fine 2D $(x, y)$-mesh. A perfectly matched layer (PML) surrounds the perimeter of the discretized grid in order to minimize reflected signals from the boundary of the grid. The thickness of this layer depends upon the boundary conditions. The custom software can adjoin the number of grids that make up the PML. The optical source used in the analysis may be located any place in the FDTD grid and consist of either transverse electric (TE) or transverse magnetic (TM) polarizations. Both types of sources, planewave or point sources, are available and have a wavelength range to match the optical materials and design criteria. Through an iterative leapfrog computation scheme, the fields of the source are time propagated in the grid. Observation points can be set up through the grid to collect field data for later analysis by Fourier transform.

As a practical example, figure 3.12 shows the calculation space of an FDTD analysis. The physical dimensions of the PhQ structure to be studied are $6 \mu \mathrm{m}$ by $13 \mu \mathrm{m}$ 
( $x$ down, $y$ across). A PML is placed around the outside perimeter of the grid and extends for an adjustable amount of grid points. The pattern extends the full $x$ direction and from $y=1$ to $y=11 \mu \mathrm{m}$ with the center of the pattern at $y=6 \mu \mathrm{m}$. The high dielectric regions of the structure are shown in black. The planewave source extends from $x=1$ to $x=5$ $\mu \mathrm{m}(y=0.25)$ on the left side of the PhQ structure. Eight observation points are placed in various locations around and inside the pattern.

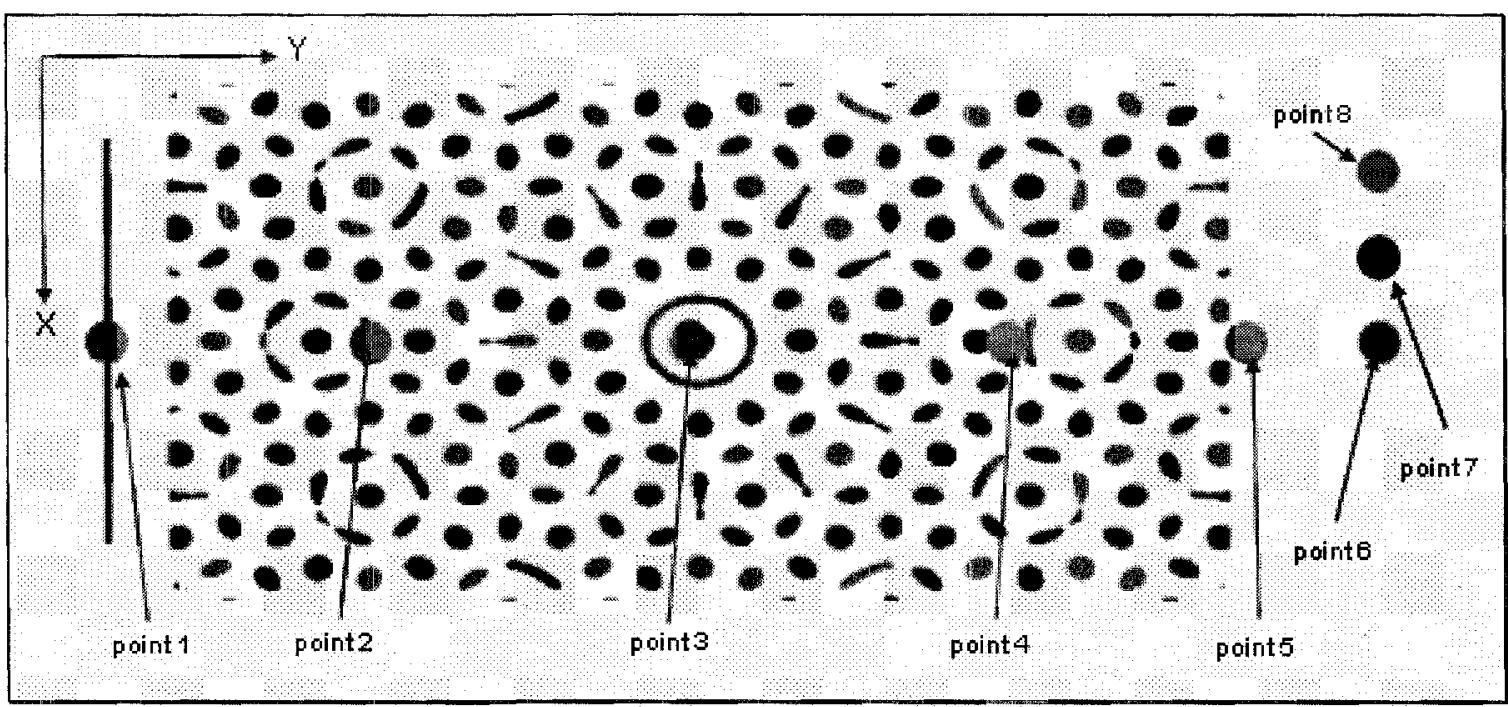

Figure 3.12. PhQ structure on a discretization grid. Black and white contrasts are related to dielectric contrasts. The solid line across shows location of the planewave source. Circles indicate observation points used for Fourier transform analysis.

For accurate transform curves to be produced, at least 100,000 time domain points are required per computational configuration. In addition, it is well known that the FDTD technique introduces phase errors as the number of grid points per wavelength is reduced. Reasonably accurate results are expected when the grid sampling density is at or above 10 points per wavelength (i.e. in the highest dielectric constant).

\subsubsection{Photonic Quasicrystal Transmission Analysis}

A reasonable PhQ pattern to start transmission analysis would be the $50 \%$ fill factor, shown in figure 3.8 (middle row, first pattern). Figure 3.13 and figure 3.14 show the 
transmission results of TE and TM polarizations respectively across a wavelength range of $900 \mathrm{~nm}$ to $1.8 \mu \mathrm{m}$. The analysis results for TE polarization shows that a small band gap exists in the vicinity of $1.05 \mu \mathrm{m} \leq \lambda \leq 1.28 \mu \mathrm{m}$ and that two defect bands at $\lambda=1.15$ $\mu \mathrm{m}$ and $\lambda=1.24 \mu \mathrm{m}$ for this propagation direction are contained. The analysis results for TM polarization shows that many large band gaps exist over the wavelength range for this propagation direction. The computations have shown that the TM polarization produces transmission plots rich in band gaps over the wavelength regime considered. The TE computations do show some transmission band gaps but are much narrower in wavelength extent, fewer in number on any single plot, and quite sensitive to the PhQ geometry and dielectric contrast. The result is tuned to how the electric field boundary conditions are satisfied within rod-like structures. In order to find physical applications for PhQs in the same way PhCs are used, one needs structures with relatively large band gaps so that one can physically add defects the structure and create waveguides or add/drop ports. A larger band gap provides better isolation for these defect modes as well.

To determine the optimal PhQ structure for generating a photonic band gap, the transmission properties were calculated over a range of fill factors. Figure 3.15 shows a 3D plot of the bandstructure versus wavelength and fill factor. Two dominant bandgap regions are easily identified. The largest gap is centered about the 1.5 to $1.8 \mu \mathrm{m}$ wavelength regime and the second is centered about the $1.0 \mu \mathrm{m}$ wavelength regime. As a result, the 20\% fill factor PhQ, shown in figure 3.8 (last row, first pattern), has the largest PBG. 


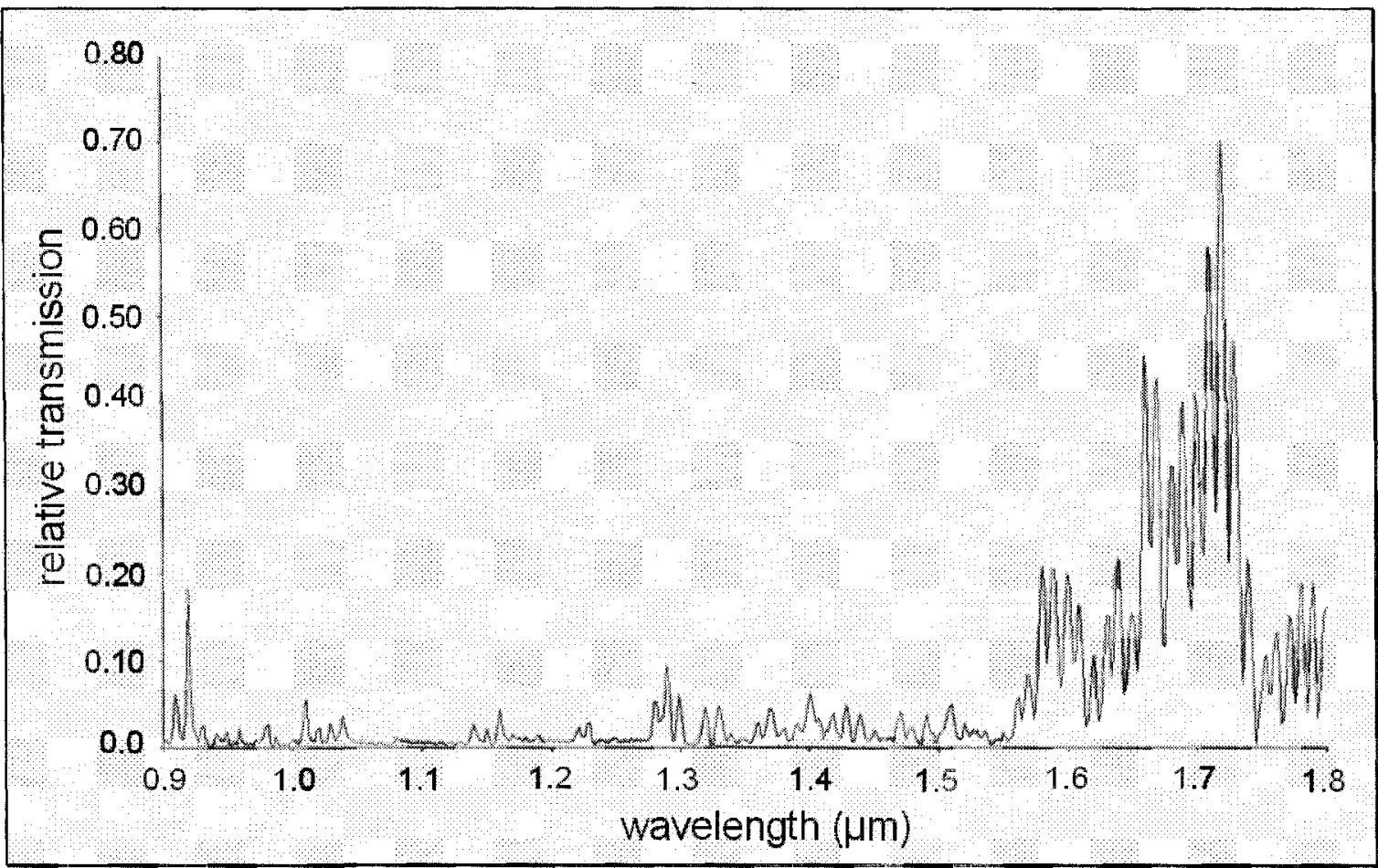

Figure 3.13. TE polarization (Hz component) transmission spectrum for a $10 \mu \mathrm{m}$ length 12 -fold PhQ of 50\% fill factor. A band gap is present between the wavelengths of 1.05 $\mu \mathrm{m}$ and $1.28 \mu \mathrm{m}$ and contains two defect states within.

In order to obtain a better picture of the bandstructure of the $\mathrm{PhQ}$, the $E_{z}$ field versus wavelength for the $20 \%$ fill factor structure versus wavelength was calculated and the results shown in figure 3.16 for various propagation angles. The transmission properties were calculated over an angle spread of 0 to 15 degrees. One can clearly see from figure 3.16 that a large PBG exists in this $20 \%$ fill factor PhQ. Structures like this one are good candidates for components in optical integrated systems because this large band gap can be exploited by inserting modes via line or point defects.

The question rests on how these PBGs occur. The PhQs are aperiodic in the direction of the transmission, and yet PBGs appear in the transmission spectra. Similar results for aperiodic structures have given band gaps in their spectra. For example, in a 1D Fibonacci chain of scatterers, the spectrum turns out to be a Cantor set with a hierarchical distribution of gaps [11]. 


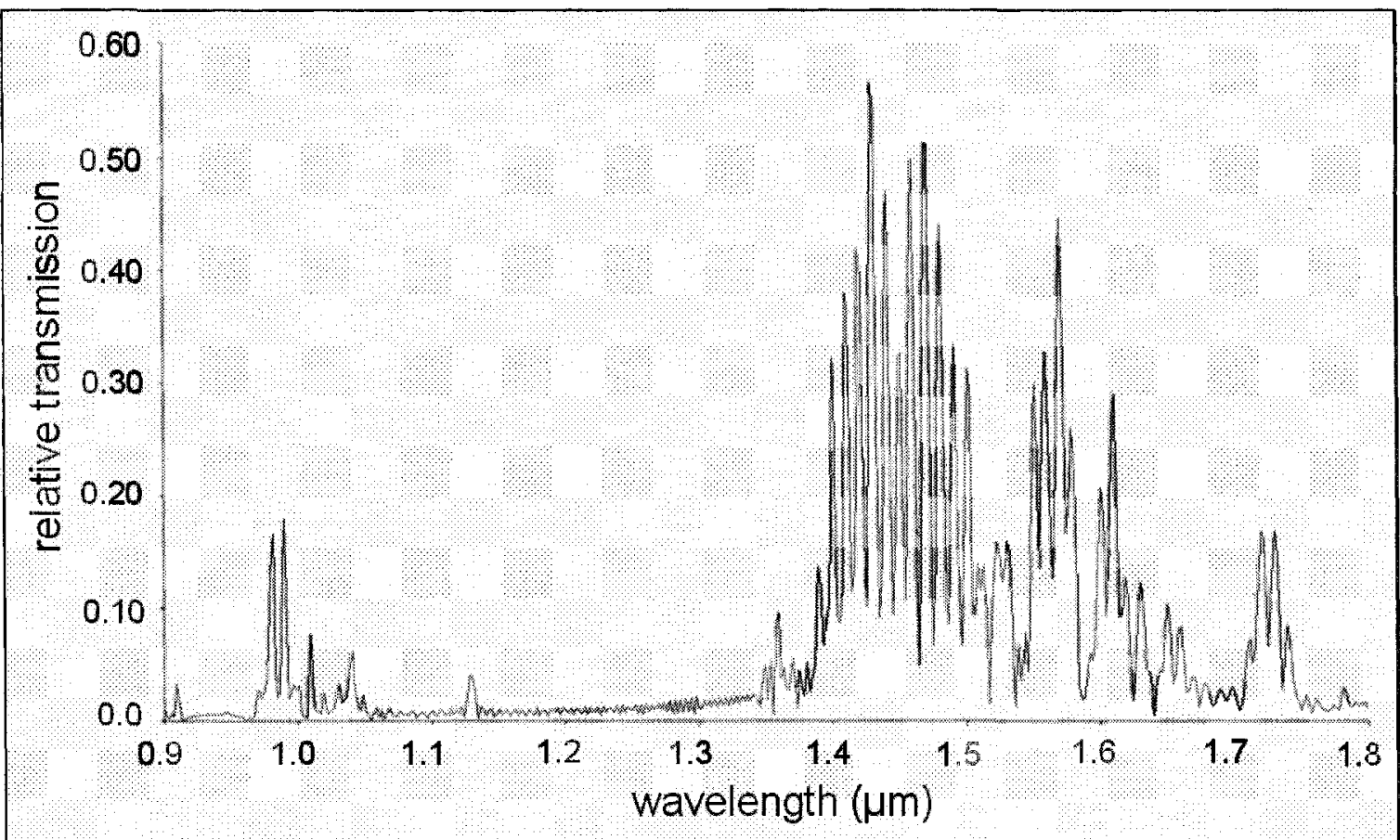

Figure 3.14. TM polarization (Ez component) transmission spectrum for a $10 \mu \mathrm{m}$ length 12 -fold quasi-crystal of $50 \%$ fill factor. Several band gap regions are present over the wavelength range displayed. Due to the rich nature of the band gaps for the TM polarization, one needs to explore in detail the optical properties of the 12 -fold quasicrystal for this polarization state of light.

In addition, 2D Penrose tilings also have given bandstructures with band gaps for both photonic and electronic systems [8].

It is clear in translationally symmetric structures that bandstructures with band gaps occur because of Bloch modes, as Chapter 2 of this thesis has shown. However, the question on how bandgaps in disordered and aperiodic structures arise in photonic systems is not at all clear. In electronic systems, tight-binding theories satisfactorily explain the formation of bandstructures with bandgaps. Tight-binding theories depend upon charged potentials. Photons are not charged, so such theories are not applicable. However, one very important point is that wave phenomena, regardless if the wave phenomena describes electrons or photons, will always localize in a $1 \mathrm{D}$ or 2D disordered or aperiodic lattice. This means that in random or aperiodic $1 \mathrm{D}$ and $2 \mathrm{D}$ lattices, one will 
have a series of localized states, and depending on whether they are degenerate or not and their relative physical locations, a propagating "mode" is merely energy propagating from localized mode to localized mode. Even though localization occurs for both disordered and aperiodic structures, how localization occurs in both these media is different. It is localization in aperiodic structures that will be addressed in this thesis.

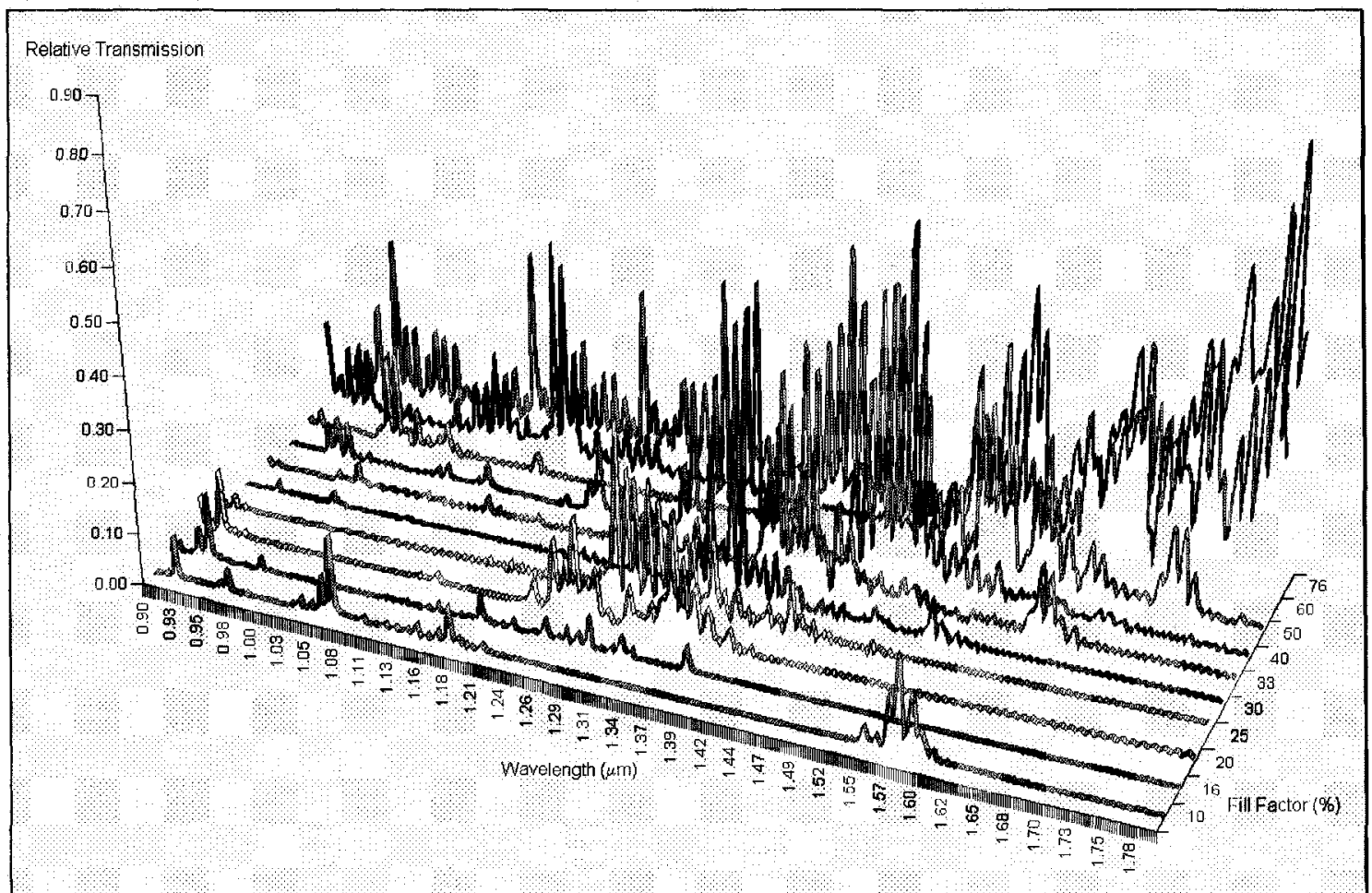

Figure 3.15. Line plot of the transmission spectrum for the Ez component of the TM polarization plotted versus wavelength and dielectric fill factor. The larger of the band gaps is located in the flat zone about the $1.5 \mu \mathrm{m}$ wavelength value. A second large band gap is located in the $1.0 \mu \mathrm{m}$ wavelength range. 


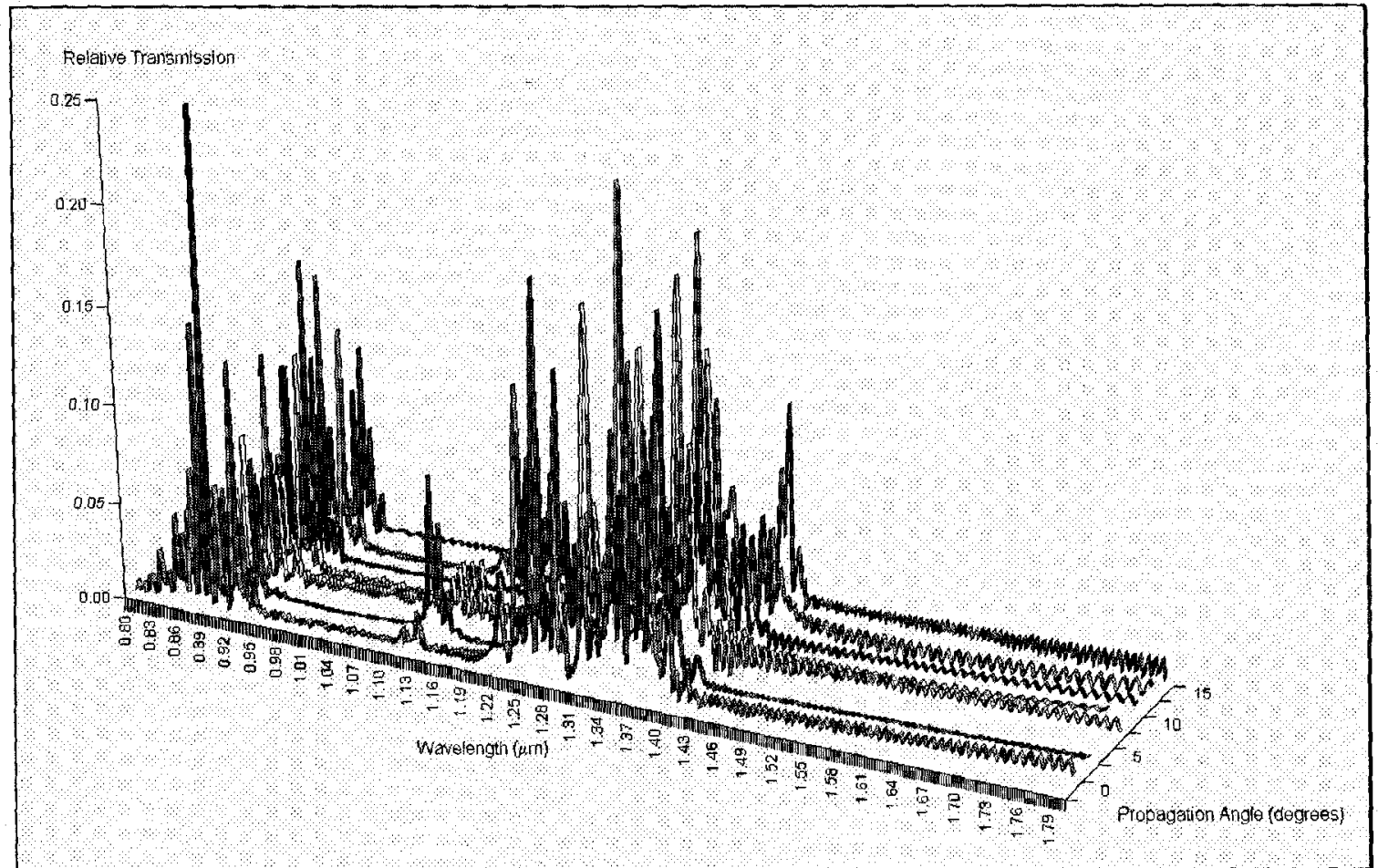

Figure 3.16. Line plot of the transmission spectrum for the Ez component of the TM polarization plotted versus wavelength and $0^{\circ}$ to $15^{\circ}$ propagation angle in $2.5^{\circ}$ increments. Through rotational symmetry, other propagation angles outside $15^{\circ}$ range can be rotated into the $0^{\circ}-15^{\circ}$ range displayed. The $P h Q$ displays a uniform transmission spectrum over a propagation angle.

\subsubsection{Photonic Quasicrystals Waveguides}

Figure 3.15 illustrates that different fill factors give different transmission characteristics It was shown that a 12 -fold $20 \%$ fill factor PhQ has the largest bandgap region between $1.43 \mu \mathrm{m}$ and $1.8 \mu \mathrm{m}$. Figure 3.16 further analyzes the $20 \%$ fill factor by studying the transmission response over a propagation angle spread. Figure 3.16 shows that the 1.55 $\mu \mathrm{m}$ wavelength is inside the bandgap. The dielectric contrast was assumed to be the largest (i.e. silicon-air). A computational study at different dielectric contrasts about the $1.55 \mu \mathrm{m}$ wavelength is seen in figure 3.17 . It shows that there is a high degree of tolerance around a $1.55 \mu \mathrm{m}$ as a center wavelength for a PBG. These simulations provided a motive for designing a PhQ optical component for $1.55 \mu \mathrm{m}$. In order to do 
this, one needs to defect the $\mathrm{PhQ}$ so that a mode at $1.55 \mu \mathrm{m}$ can be guided via line defects, and added or dropped via point defects. The defect properties of PhQs have already been well characterized in the literature [19]. It shows that they have richer defect qualities and are more wavelength-selective than defects in PhCs. These two qualities alone make $\mathrm{PhQs}$ lucrative candidates for components of a next-generation DWDM optical system.

The first defect type to study is the line defect or waveguides. Waveguides can be introduced in many different ways, such as a rectangular region of removed rods, or filling that rectangular region by high dielectric material. The introduced waveguides may have any orientation with respect to the crystal structure.

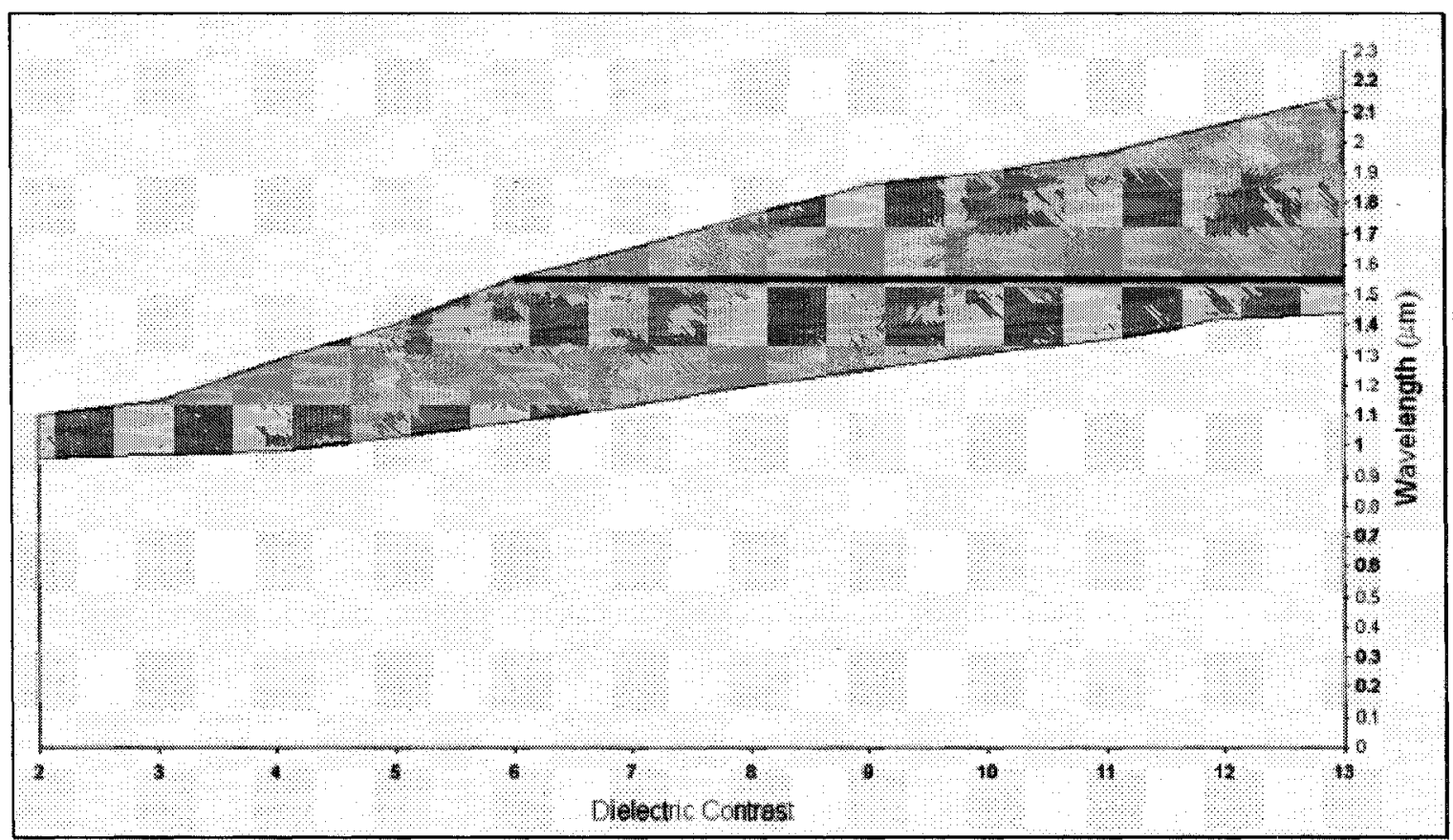

Figure 3.17. Dielectric contrast vs. band gap center wavelength. Dark region shows the relative size of the band gap.

Because of the lack of translational symmetry of the quasicrystal pattern, the location of the waveguide with respect to the central region (dot and ring of figure 3.12 ) is an important design feature. This implies that given the shape, orientation, and dielectric 
value of the waveguide, its placement within the quasicrystal structure can influence the optical guiding properties of the waveguide. This is a feature not available in conventional PhC arrangements, and can be exploited by a careful and skillful optical engineer. Straight rectangular waveguides were introduced into the PhQ of figure 3.12. The waveguides were chosen to have a relative dielectric value of one, and extended the full width (left to right) of the PhQ. The longitudinal axis of the waveguide went through the center part of the PhQ. The analyzed waveguides had widths ranging from $0.25 \mu \mathrm{m}$ to $1.5 \mu \mathrm{m}$. The crystal and waveguides were extended to $18 \mu \mathrm{m}$ and the discretization grid extended to $6 \mu \mathrm{m}$ by $20 \mu \mathrm{m}$. For any waveguide, the source extent was made to equal the waveguide width. A continuous multi-wavelength source was propagated through the structure grid and the field values were recorded at several locations along the waveguide and on the waveguide output.

A plot of the Poynting vector magnitude versus wavelength and position is shown in figure 3.18 for the $1.50 \mu \mathrm{m}$ wide waveguide. The wavelength range extending from $1.4 \mu \mathrm{m}$ to $1.8 \mu \mathrm{m}$ shows a high transmission. This same range corresponds to the large bandgap of figure 3.15 and figure 3.16. The light in the low dielectric waveguide is confined to the waveguide, as it cannot propagate within the PhQ. In the range of 1.2 to $1.4 \mu \mathrm{m}$, the waveguide transmission is low and results from the light in this wavelength range bleeding into the PhQ. The waveguiding properties in the 1.4 to $2.2 \mu \mathrm{m}$ range is further examined by plotting field plots of the propagations and introducing waveguides of various geometry.

Figure 3.19 shows the optical propagation of $1.50 \mu \mathrm{m}$ light through the $1.5 \mu \mathrm{m}$ wide straight waveguide. A planewave is excited at the input (left) side of the waveguide 
and its propagation is modeled through FDTD. As can be seen in the plot, the light is well confined to the waveguide with little loss or leakage into the PhQ. The low loss wave propagation for this wavelength is confirmed in the traces shown in figure 3.18.

The above analysis uses a low dielectric waveguide defect for propagating an optical wave through the 12 -fold $\mathrm{PhQ}$. One could use a high dielectric waveguide as well, as shown in figure 3.20 , but the effect of the PBG is not as obvious because light tends to look for lower energy states in high dielectric regions. In the low dielectric waveguide case, the light is propagating in a low dielectric region surrounded by high dielectric rods. This truly shows that the light is confined to a low dielectric region because lower energy states in high dielectrics are inaccessible due to the bandgap region of the PhQ.

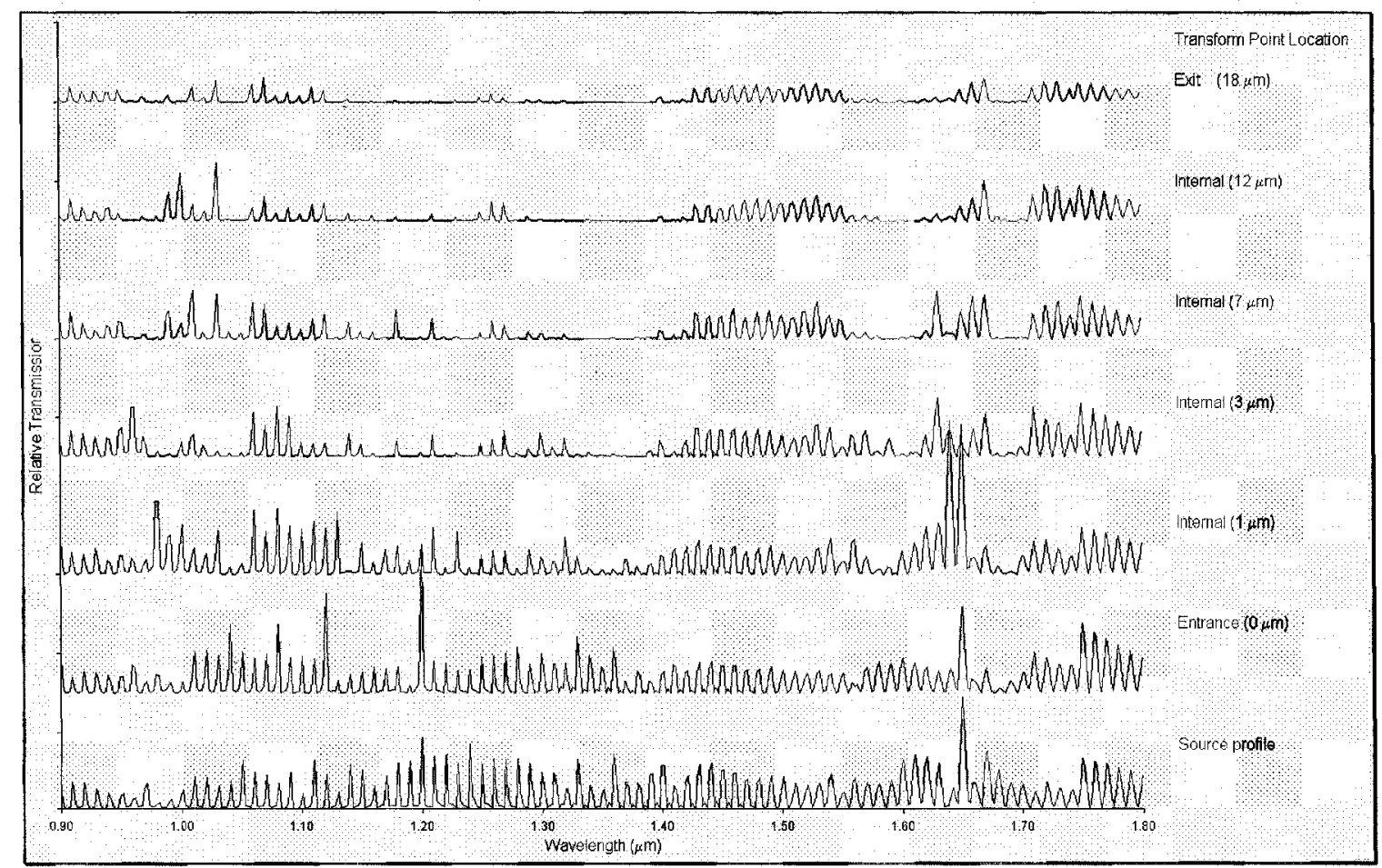

Figure 3.18. Plot of the Poynting vector magnitude vs. wavelength and Fourier transform point location in the center of a $1.50 \mu \mathrm{m}$ wide waveguide centered on the PhQ. The top trace indicates that the low dielectric waveguide transmits strongly in the band gap range of the quasi-crystal and leaks light in those wavelength ranges which correspond to the transmission bands of the PhQ. 


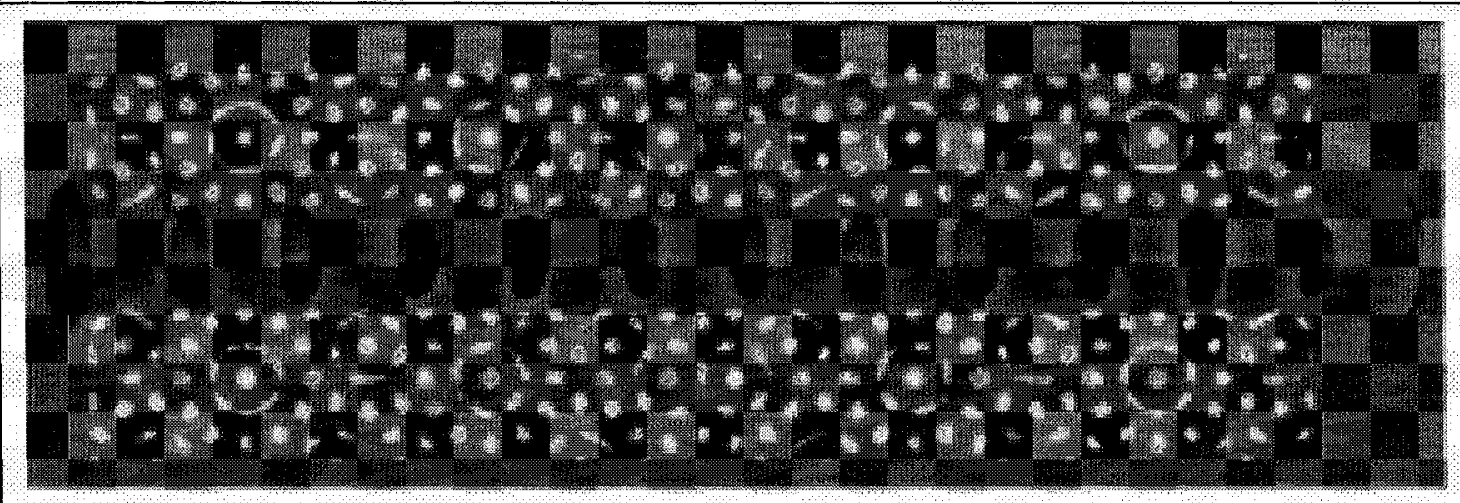

Figure 3.19. Transmission profile of the $1.5 \mu \mathrm{m}$ wavelength through a $1.50 \mu \mathrm{m}$ low index waveguide centered on the PhQ. The dimensions of the PhQ is $6 \mu \mathrm{m}$ across and $17 \mu \mathrm{m}$ long. High transmission is observed for this wavelength.

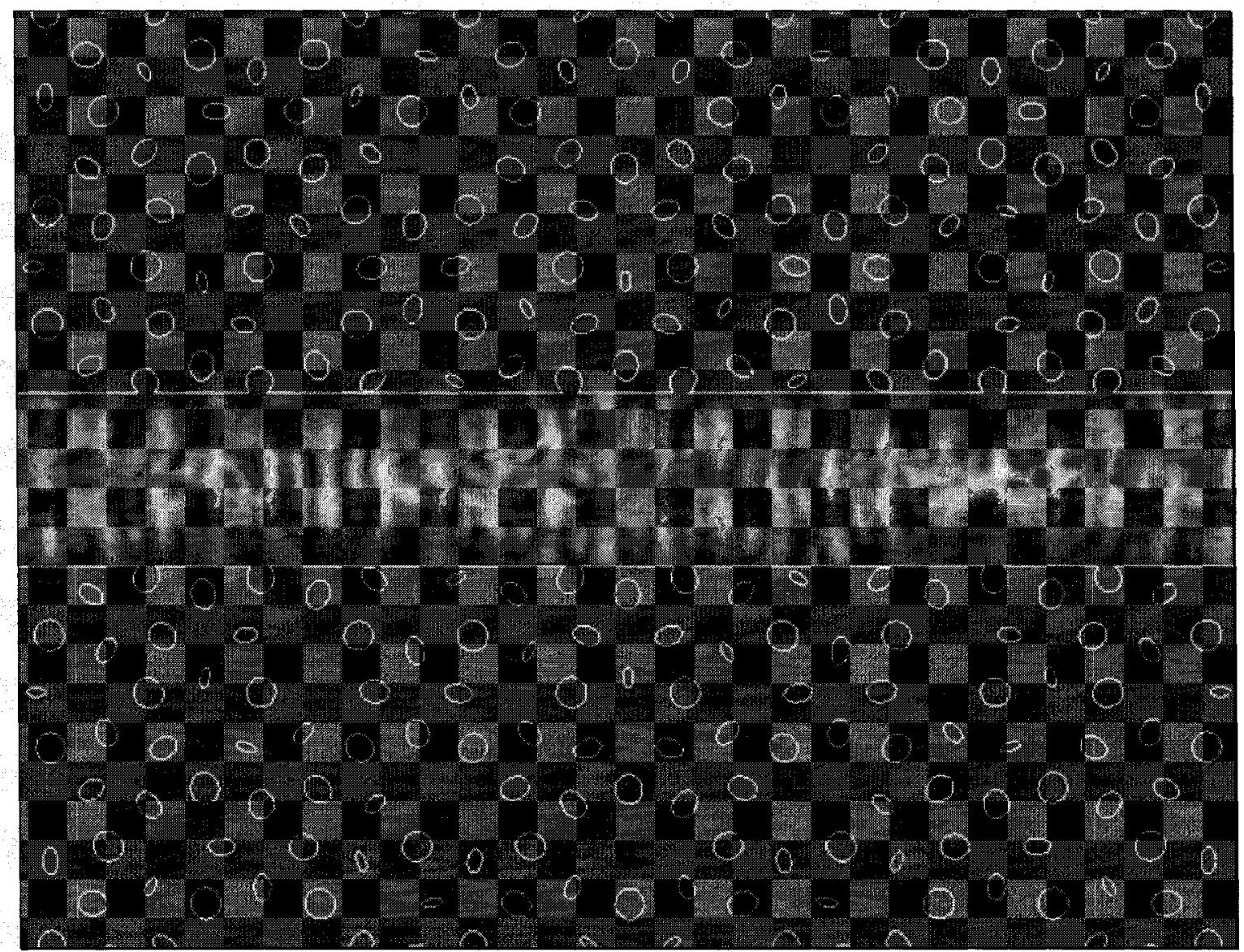

Figure 3.20. High dielectric waveguide through 12-fold $20 \%$ fill factor $\mathrm{PhQ}$.

In reality, making such a line defect in a low dielectric would lead to large out-of-

plane losses, and therefore, for functional purposes all waveguides that will be built will be high-dielectric material waveguides. One could back fill the air gaps in the PhQ with 
an index larger than air but lower than the rods, although all the work in this thesis is focused on air-silicon dielectric constants.

\subsubsection{Towards a Photonic Quasicrystal Device}

Figure 3.21 illustrates a procedure for using the properties of PhQs to design a photonic device for DWDM applications. This procedure exploits the fact that different transmission qualities arise from different fill factors, as shown in figure 3.15. This implies then that the wavelengths coupled to the defects will be different. This feature, coupled with the fact that PhQs have defect states that are more wavelength-selective than $\mathrm{PhCs}$ [19], yields a very efficient wavelength add/drop functionality for DWDM optical circuitry. Furthermore, as discovered in the previous section, line defects can be used over a common wavelength. Hence, one could then take portions of different fill factor PhQs, stitch them together via their common line defect (i.e. waveguide), and have their own unique point defects add or drop different wavelengths. If the stitching can be turned into a pattern grading, finer control over wavelengths can then be achieved.

Each of the four slice-regions in figure 3.21 that come together to make the graded PhQ need to be modeled individually. Before adding the defects, the slice-regions need to be characterized via their transmission results. This can be done by implementing a novel moveable computational domain window (MCDW) in the FDTD software. 


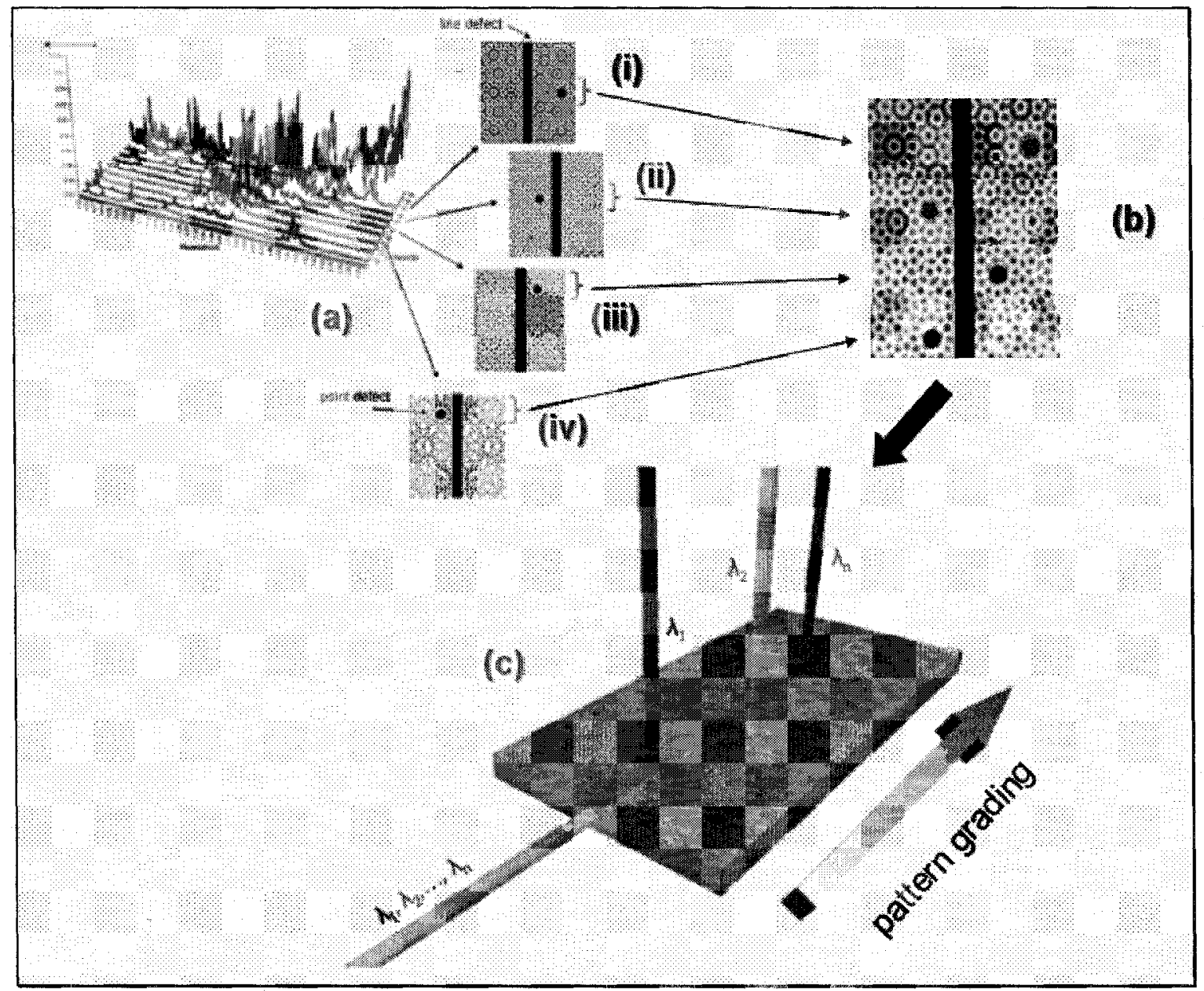

Figure 3.21. Process towards PhQ DWDM Device. (a) Figure 3.15 indicates that different fill factor PhQs have different transmission characteristics. Line defects about a common wavelength can then be placed in each PhQ and different point defects with different resonance wavelengths can be designed. (b) Take a portion from each PhQ fill factor pattern and stitch pattern via the common line defect. (c) Continuous stitching or grading of (a) and (b) process to gain finer wavelength, $\lambda_{\mathrm{n}}$, add/drop control.

\subsubsection{Moveable Computational Domain Window}

The MCDW was invented [41] to probe transmission characteristics of PhQs in different regions of the PhQ. The width of this window is adjustable and it can be moved from one region of the structure to another with the FDTD computation only occurring with the MCDW. Figure 3.22 shows a diagram of the MCDW. 


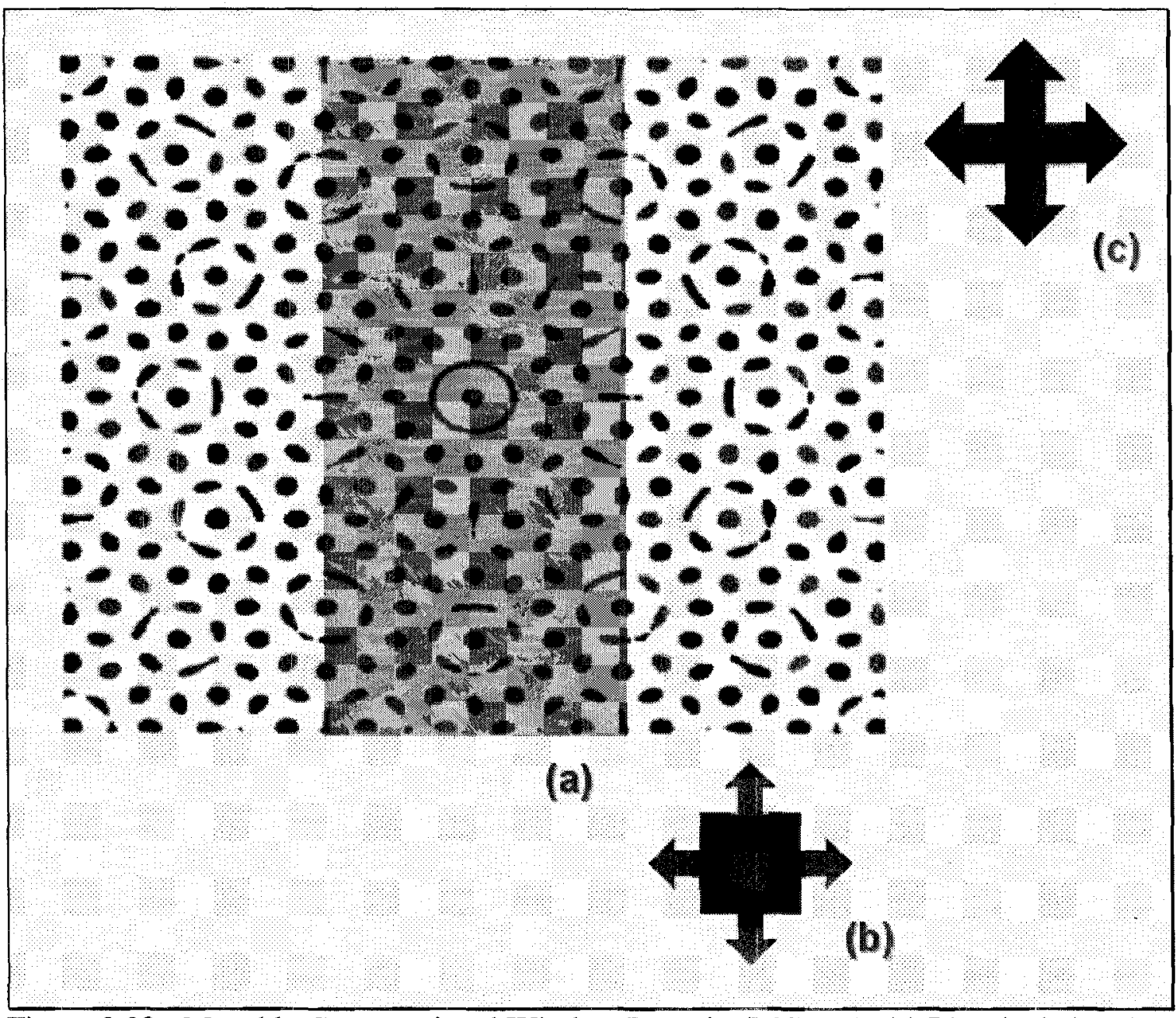

Figure 3.22. Movable Computational Window Domain (MCWD): (a) The shaded region in the PhQ pattern is the MCDW. (b) Arrows indicate that the MCWD can be scaled in size and (c) Arrows indicate that it can be moved around within the pattern. All FDTD features and analysis occur within this window only.

The MCDW was applied three times to the $20 \%$ fill factor PhQ with three different widths, as shown in figure 3.23, figure 3.24 and figure 3.25. In addition, the MCDW was offset from the center of the PhQ pattern to calculate the bandstructure of different areas within the pattern. Figure 3.23 shows the results of a $10 \mu \mathrm{m}$ MCDW. The structure has a very large stopgap region even when the center of the MCDW is offset $15 \mu \mathrm{m}$ from the center of the pattern. Figure 3.24 and figure 3.25 show similar results for $5 \mu \mathrm{m}$ and $3 \mu \mathrm{m}$ MCDWs, respectively. 


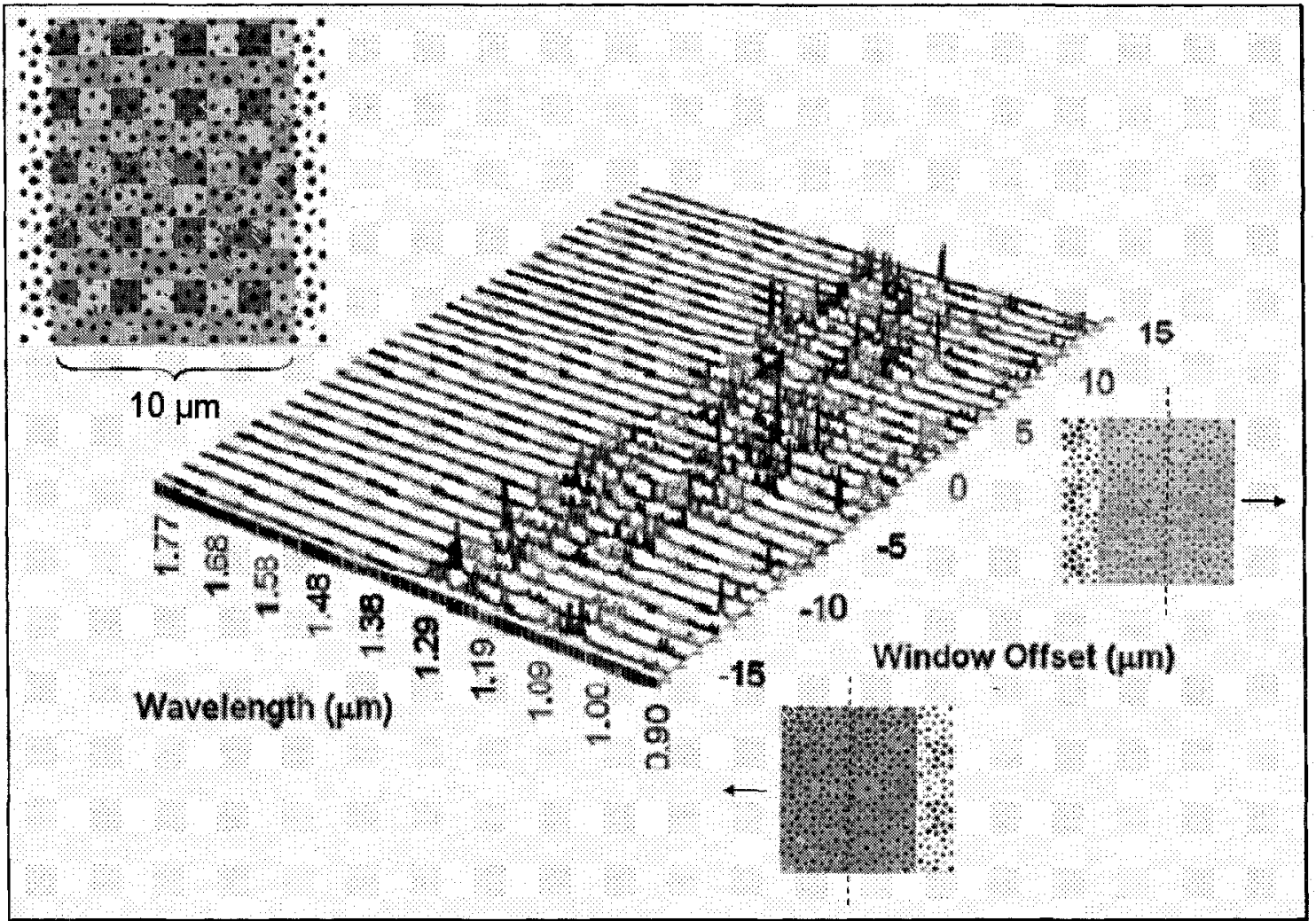

Figure 3.23. Transmission spectrum for MCDW of width $10 \mu \mathrm{m}$ in 12 -fold $\mathrm{PhQ}$.

The large bandgap is still well resolved even when the slice width is as small as $3 \mu \mathrm{m}$. However, for this width, several local maximums in the field component are observed in the otherwise bandgap region. These local maximum wavelengths are dependent on slice location in the PhQ pattern and may be regarded as local effects in much the same way as defects are considered in otherwise perfect translational PhCs. Figure 3.23, figure 3.24 and figure 3.25 confirm the belief that some bandgaps in PhQs are generated via local effects as alluded to in Chapter 2.

Other transmission plots of different fill factor PhQs have been published [39] and they all show similar results for thin slice MCDW analysis: local field maximums within the bandgaps that appear to be similar to defect states in PhCs. 


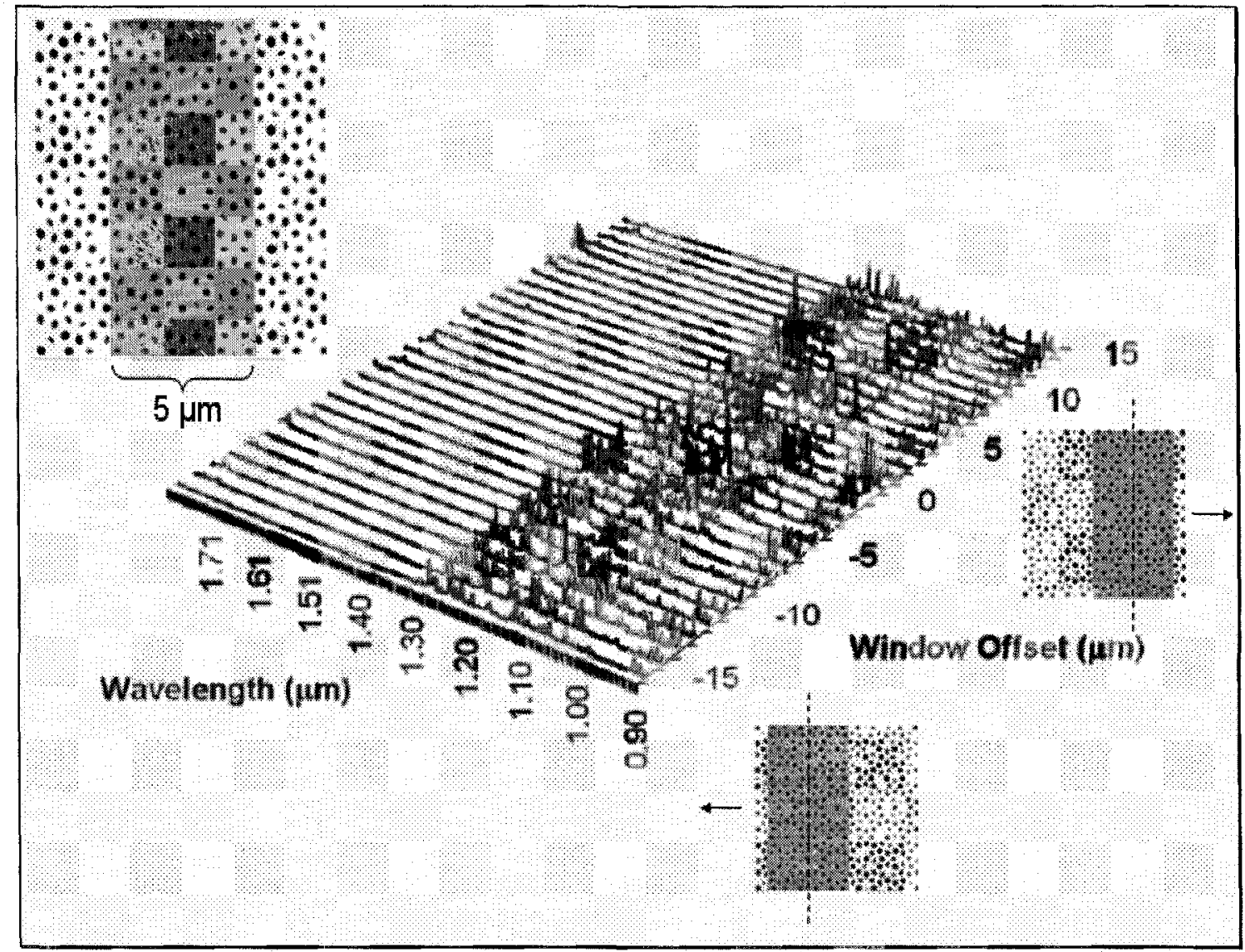

Figure 3.24. Transmission spectrum for MCDW of width $5 \mu \mathrm{m}$ in 12-fold PhQ.

This result implies that defect states in PhQs can be created in a different way: instead of introducing physical abnormalities such as removing rods or changing the material properties in a local area, one can change the sample size (i.e. physical size of the pattern to be designed) of the PhQ and therefore introduce defect states. This relationship between state localization (i.e. defect states) and sample size is already present in disordered media. However, with disordered media, localization occurs when the sample size grows larger, while for PhQs, localization occurs when the sample size shrinks. Hence, creating localized states in PhQs in this way requires a decper analysis. 


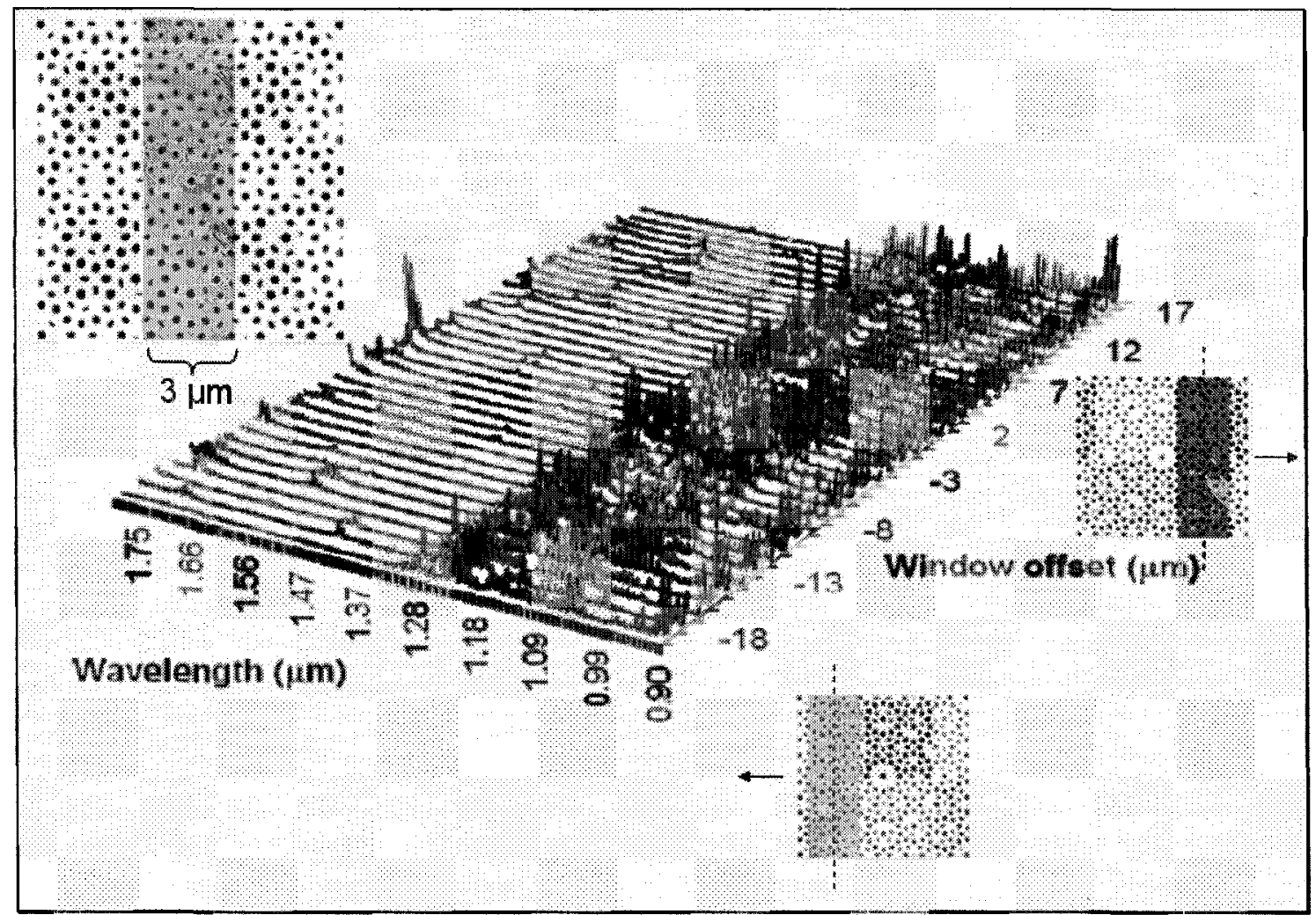

Figure 3.25. Transmission spectrum for MCDW of width $3 \mu \mathrm{m}$ in 12-fold PhQs.

\subsubsection{Photonic Quasicrystal Defect State Analysis}

Figure 3.26 shows a comparison between the transmission response of the $10 \mu \mathrm{m}$ (upper trace) and $3 \mu \mathrm{m}$ (lower trace) slice widths with no offsets. For the $3 \mu \mathrm{m}$ transmission response, there is a local transmission region in the $1.56 \mu \mathrm{m}$ to $1.59 \mu \mathrm{m}$ wavelength range which is located in the large bandgap region of the $10 \mu \mathrm{m}$ slice width (upper trace). The circles in the lower trace identify defect states that were not present in the upper trace. A possible reason why these sample-size-dependent defect states are present in the $3 \mu \mathrm{m}$ slice but not in the $10 \mu \mathrm{m}$ slice is because different regions of the $\mathrm{PhQ}$ have different bandstructures. This is implied by the results shown in figure 3.25. For example, the first $3.5 \mu \mathrm{m}$ slice region in the $10 \mu \mathrm{m}$ area would have blocked out these defect states. 


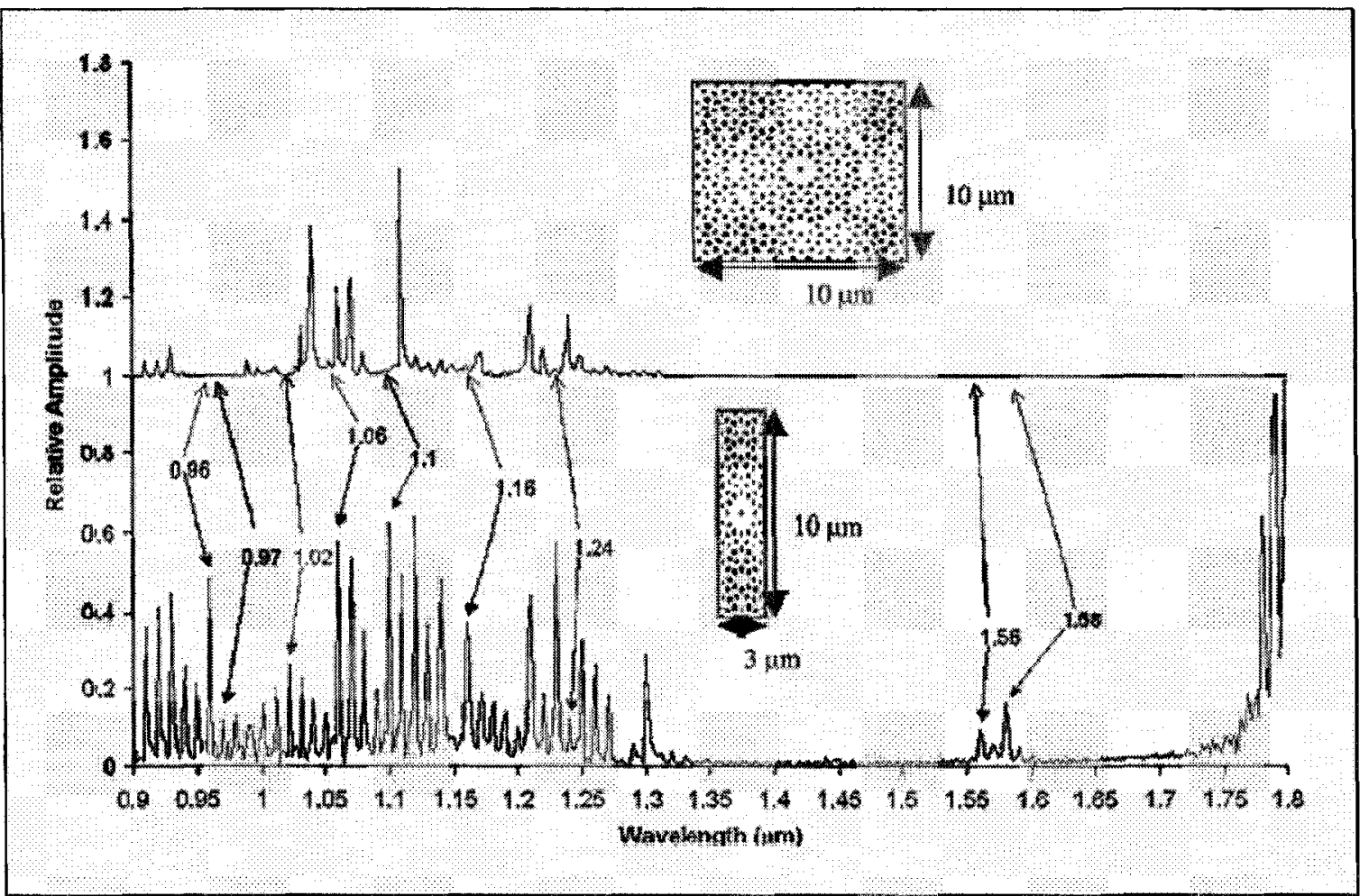

Figure 3.26. Sample size FDTD transmission runs of PhQ. Top trace shows large stop gap region of $10 \mu \mathrm{m} \mathrm{PhQ}$. Lower trace shows that 'defect' states have occurred because of sample size change to $3 \mu \mathrm{m}$. The solid circles show 'defect' states that were not present in the $10 \mu \mathrm{m}$ and the dashed circle shows that are perhaps in-band defect modes that have been made stronger because of sample size.

From the above observations, one has to note that these defect states were not physically introduced into the pattern like regular PhC defects. They were always there in the 12-fold dodecagonal PhQ lattice. In other words, there are defect states in defectfree quasicrystal lattices. These defect states can also be called localized states.

This interesting feature may hold the key to reasons why PhQs have bandstructures in the first place, and this feature may be described as an optical tightbinding theory, which parallels the electronic tight-binding theory that solid-state theorists use to describe the band structure of electronic quasicrystals and amorphous materials. This may seem puzzling at first because tight-binding models are based on bonds, and photons do not form bonds like electrons. Before fully explaining this, these localized modes should be examined further. 
For a closer examination of these states, the $\mathrm{PhQ}$ pattern is placed in a $9 u \mathrm{~m}$ diameter FDTD computation domain with a noise source placed at the center of the PhQ pattern, as shown in figure 3.27. This source excites all possible wavelengths and propagation constants in the PhQ. Localized modes coupled to the central region (or other regions) will remain when the steady state is reached after a large number of FDTD time iterations. After several hundred thousand iterations, an $E_{z}$ field profile is observed to show a high degree of rotational symmetry, even though the level of rotational symmetry changes from one level to the next as the iteration process continues. This pattern evolution indicates that there are several modes of oscillation coupled to the center of the quasicrystal and these modes are present even after the source has long since terminated its contribution to the energy of the system. The wavelength of the modes and their confinement to the center can be determined by placing observation points within the center region of the pattern and outside the pattern near the PML boundary. Figure 3.28 shows the results of this analysis.

Several maxima are observed in this plot with the dominant mode occurring at the wavelength of $1.56 \mu \mathrm{m}$, corresponding to the central maxima in the large bandgap of figure 3.26. Other maxima, which were not originally in the bandgap, are observed in this trace and correspond to other modes localized about the central region of this $\mathrm{PhQ}$ pattern. Examination of these particular modes indicate that a transmission of the wavelength is present for the thin slice and is absent for the thick slice throughout the PhQ pattern. These particular wavelengths couple to states similar to the defect states of the large band gap region. 


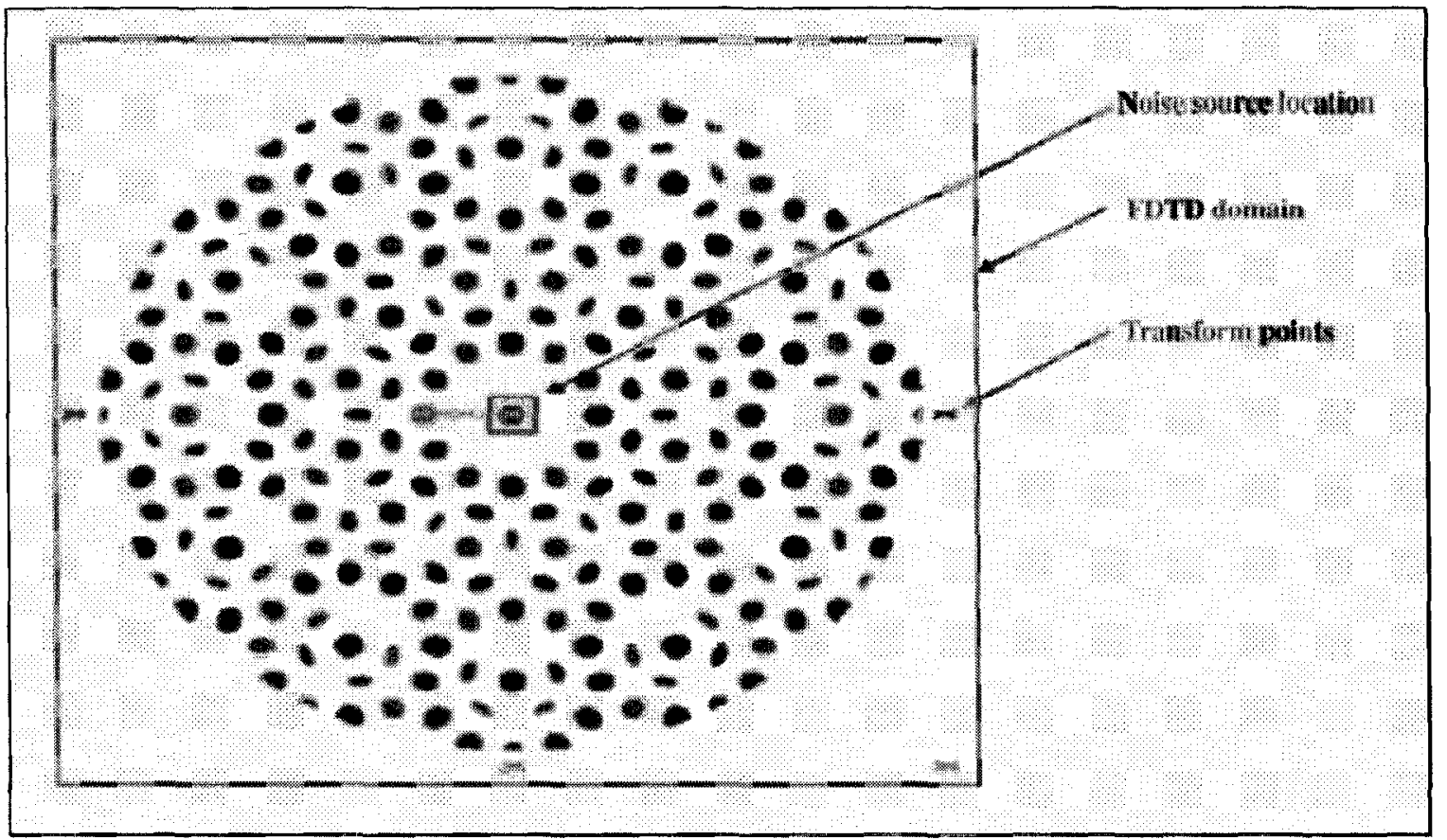

Figure 3.27. FDTD domain for analysis of localized states.

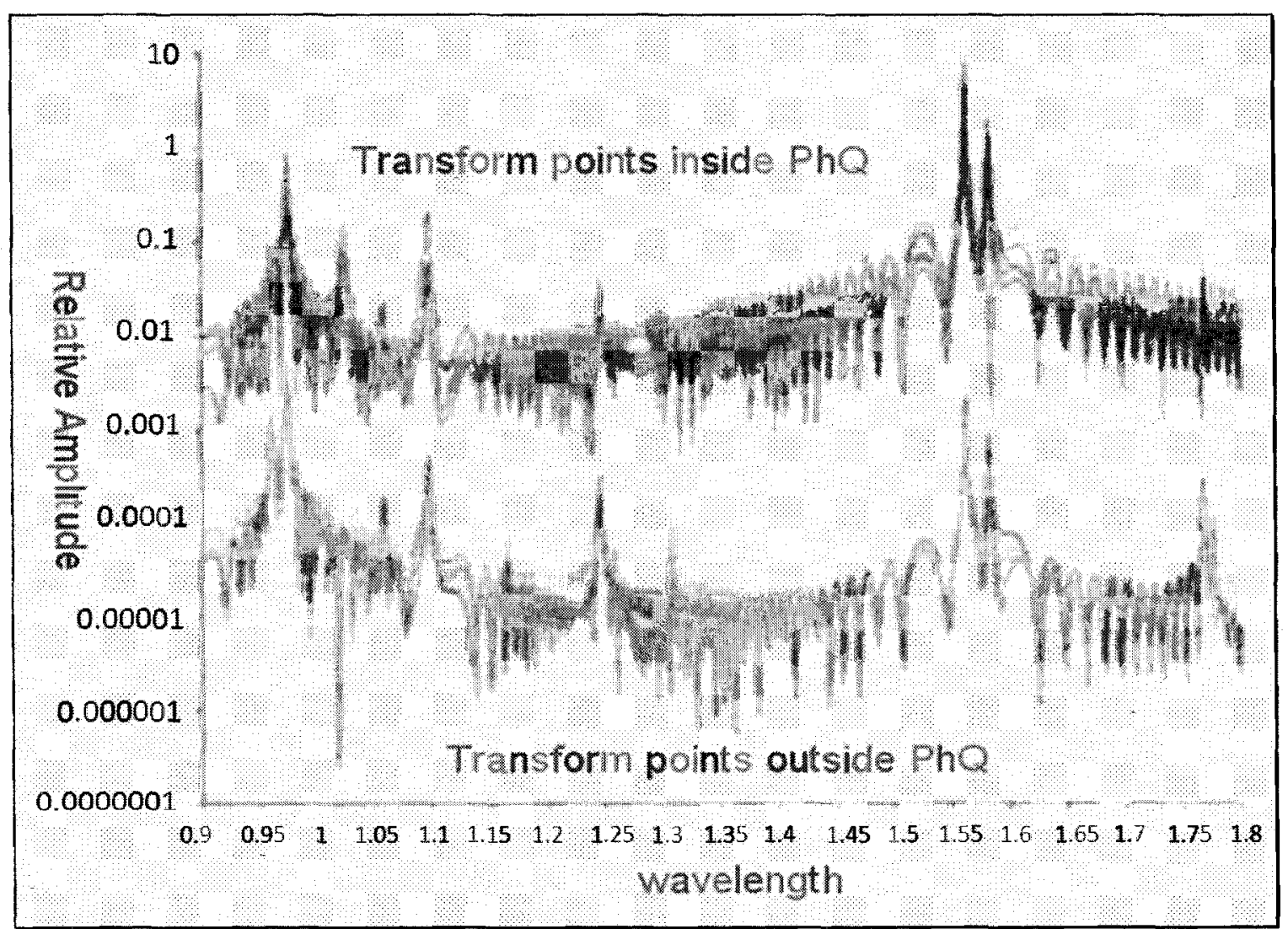

Figure 3.28. Fourier transform of observation points of FDTD defect state analysis inside and outside $P h Q$ pattern. 
It seems that these defect modes are comprised of two types of localizations: localizations in the band region of the $\mathrm{PhQ}$ and localizations in the band gap regions.

Confinement of these defect states is strong because the field amplitudes for the observation points external to the $\mathrm{PhQ}$ are several orders of magnitude lower than the amplitudes of the observation points in the central region. Figure 3.29 shows the mode profiles and where they occur in the transmission spectrum.

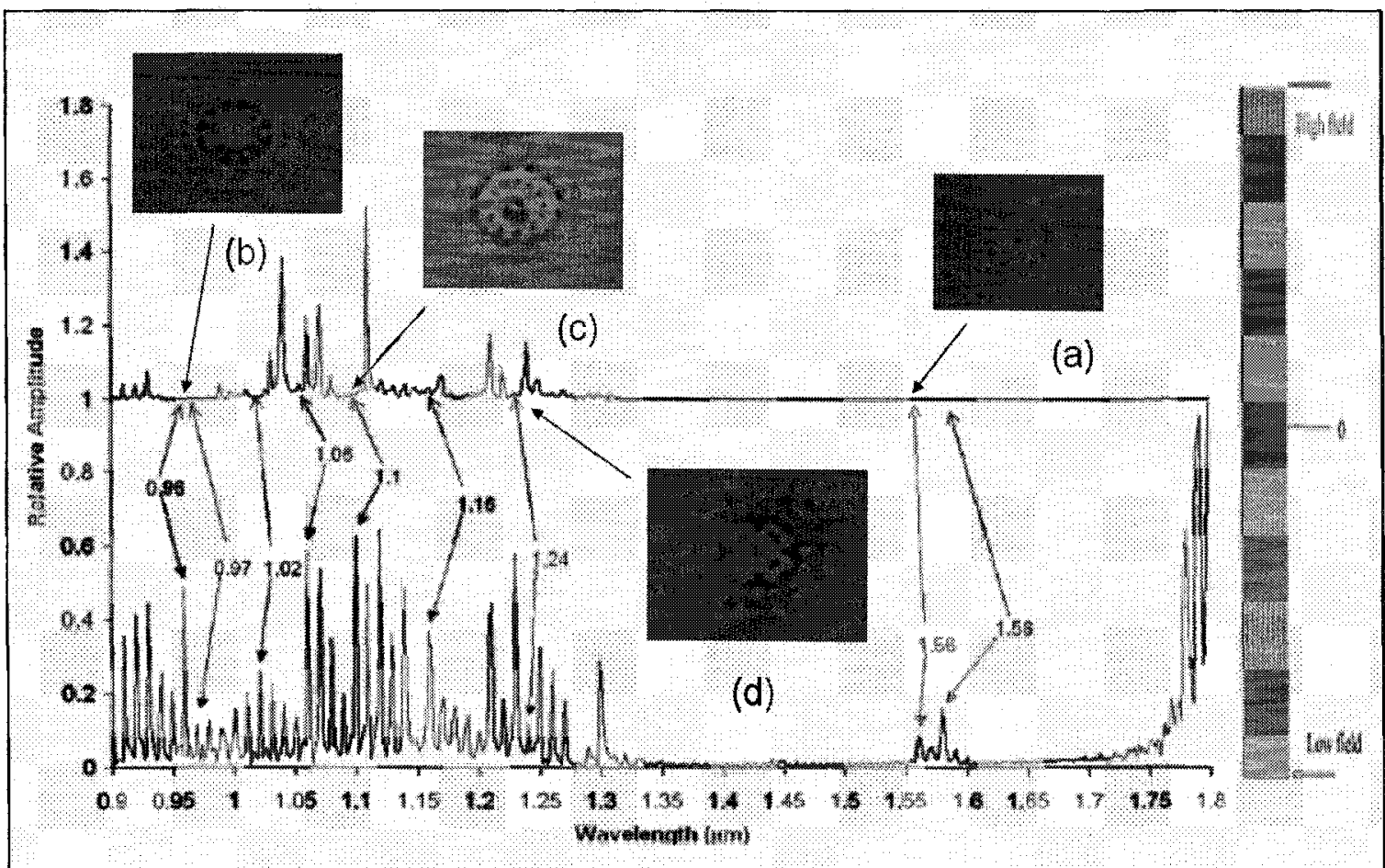

Figure 3.29. Defect mode profiles and where they occur in the transmission spectrum. (a) occurs in band gap at 1.56 um, (b) occurs in smaller band gap at $0.96 \mathrm{um}$, (c) occurs in band at $1.1 \mathrm{um}$, and (d) occurs in band at $1.24 \mathrm{um}$.

Profiles (a) and (b) are defect states that occur in band gap regions when the sample size is a $3 \mu \mathrm{m}$ slice. Profiles (c) and (d) seem to be already present yet are enhanced when the sample width is thinner. These results imply that there are two types of localizations in PhQs: (1) bandgap localizations and (2) bandedge localizations. Bandgap localizations seem to be unique to PhQ lattice symmetries [16] while bandedge localizations have been found in both disordered [16] and PhQs [8]. 


\subsubsection{Crystal Angular Momentum (CAM) Postulate}

There are two papers in the literature that took note of these states. The first paper, Chan et al. [8], was the first to coin the term photonic quasicrystal. They noted that PhQs had bandstructures and that the locations of the large band gaps in the DOS calculations did not change much even when the PhQ's domain became smaller. That is, only a local area was analyzed with the far regions represented as an averaged dielectric region. They noted that the bulk of the bandstructure was created from this local phenomena. They also noted localized sites not in the band gap region, but in the bandedge region or the region of high DOS. It is worth quoting their work here:

"Although our system is too small for a quantitative characterization of the localization property of these states, the results show that photonic "quasicrystals" can open another possibility of realizing the localization of photons, and the localization behavior may be quantitatively different from that of disordered media. We note that these highly localized states have nothing to do with defect or disorder." The structure they studied, shown in part (a) of figure 3.30, did not have any physical defects, and its localized mode was not in the gap of the DOS curve, but rather in the bandedge. Another paper, Wang et. al. [9] also found localized states when there were no defects added to the PhQ, and these states were found in the bandgap regions, as indicated by the results shown in figure 3.31(b). They went further and showed experimental evidence of these structures using microwaves. Wang et al. looked at dodecagonal PhQs and physically found bandgap and bandedge localized states similar to Chan, but were not able to find such states for octagonal or decagonal PhQs. Note that Chan's structure was octagonal while Wang's structures were dodecagonal. 


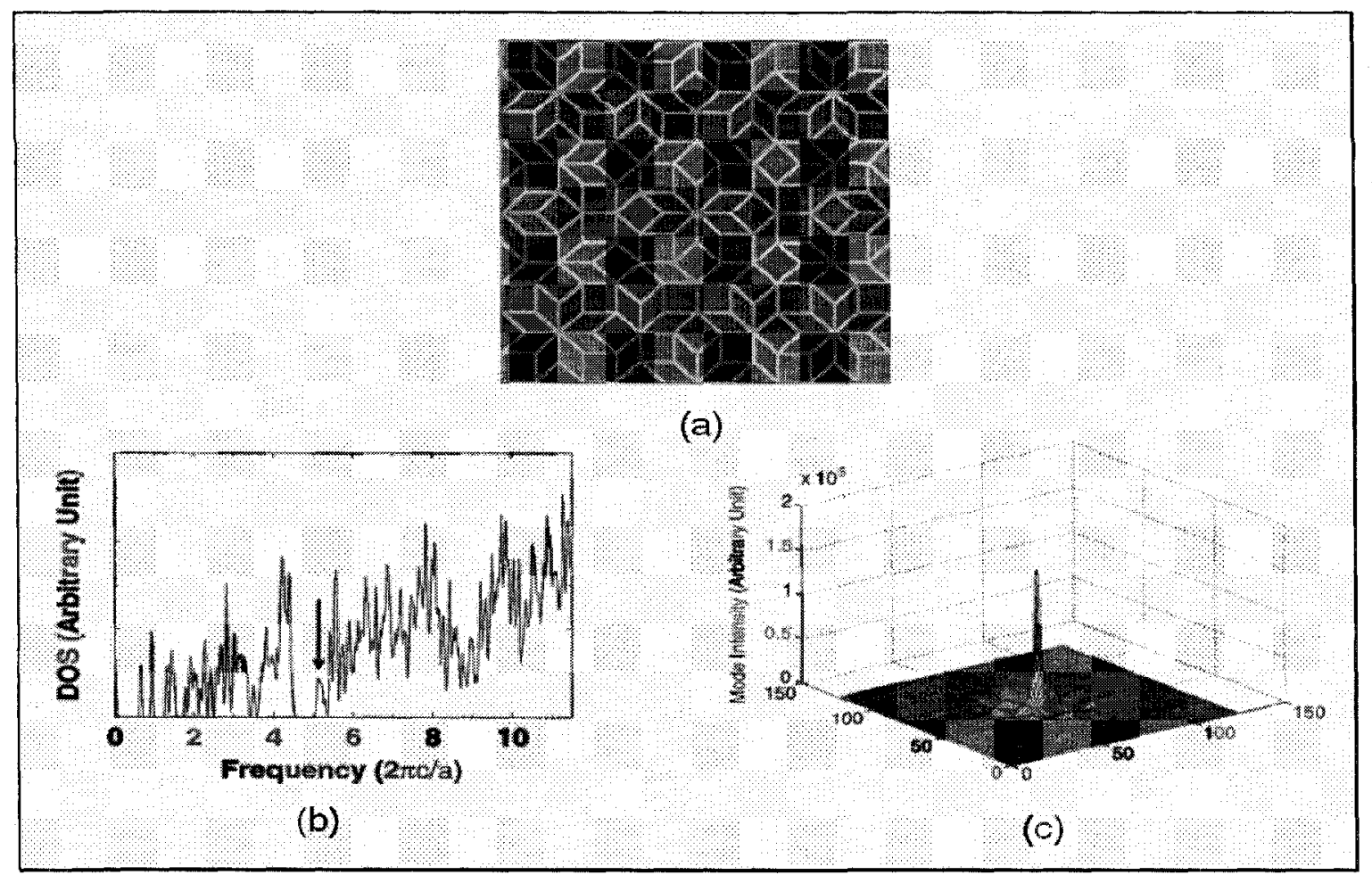

Figure 3.30. Results from [8]. (a) Eight-fold structure with gaps in DOS curve, (b) DOS curve showing frequency of (c) localized state profile.

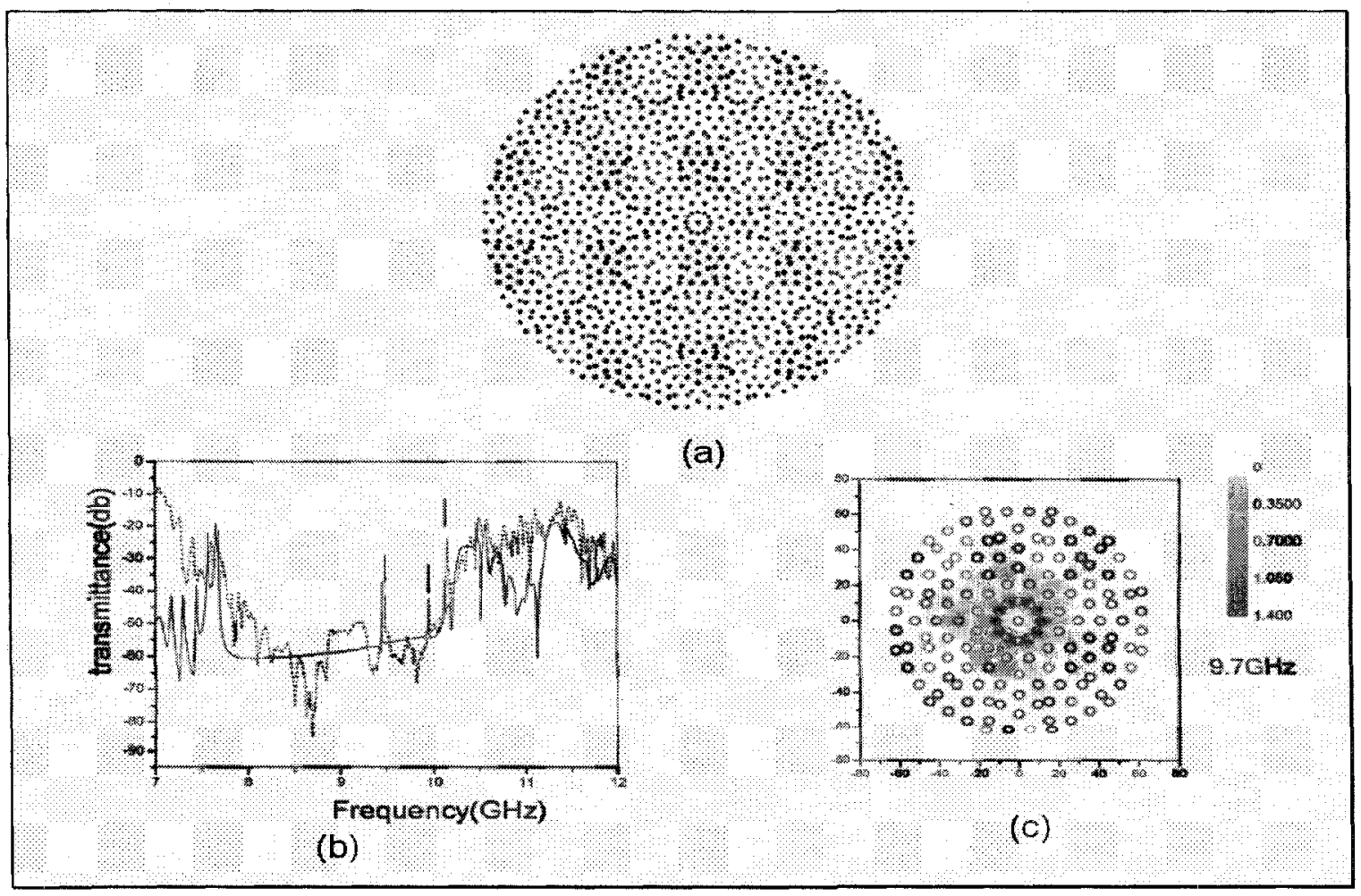

Figure 3.31. Results from [9]. (a) Dodecagonal PhQ structure made from circular rods, (b) is the comparison between simulated and measured, where arrows point to localized modes and (c) is an example of localized modes found. 
The author of this thesis postulates the reason for this discrepancy between these two published results: localization mode existence depends upon the scatterer form factor, which selects extended mode solutions of the rotational symmetry generated by a crystal angular momentum (CAM). In other words, the rotational symmetry of the quasicrystal (i.e. the rotational foldness of the crystal, which is equal to $2 \pi / n$, where $n$ is the fold number) can be identified with an orbital angular momentum eigenvalue $l$ that yields $2 l+1$ states, and these states are only made available via the scatterer form factor of the crystal or quasicrystal. In the case of PhQs, the scatterer form factor is identified with the dielectric form factor (DFF) defined in Chapter 2. These CAM states are the analogies of Bloch states in translationally symmetric structures. They appear to look like localized defect states, but are, in fact, extended in some kind of rotational space. If the DFF basis is shaped correctly, then all these modes can be exhibited. However, selecting such a crystal basis is difficult but possible, as will be shown in the next section.

Hence, the reason why Chan et al. found localized states for an octagonal $\mathrm{PhQ}$ and Wang et al. did not, is because Wang at el. was using circular rods, while the structure studied in Chan et al. (figure 3.30(a)) used linearly connected type structures. The postulate in this thesis states that there would be $2 l+1=2(8)+1=17$ states available for octagonal lattices, since an octagonal structure has an 8 -fold or $2 \pi / n=2 \pi / 8=\pi / 4$ or $45^{\circ}$ rotational symmetry. The shape of DFF then selects from these states and makes them available for incoming light states.

In order to study these CAM states further, one requires a numerical eigenvalue solver that can analyze structures with complicated shapes. This numerical technique is known as the finite element method (FEM). 


\subsection{Finite-Element Method (FEM) Technique}

Generally, when approaching a new problem, it is best to apply the FDTD method to get an understanding of how a system responds to a given stimuli. In this case, the FDTD analysis in the previous section revealed a new phenomenon that is connected to localized states in PhQs with no physical defects. The results lead the author to postulate the existence of CAM states and their dependence on the DFF. Since the CAM states are related to the rotational symmetry of the system, and symmetries are related to eigenvalues, a detailed eigenvalue approach is now warranted. In addition, the dependence on the DFF has shown that scatter shape is important. These two reasons, (1) eigenvalue analysis required and (2) shape dependence, make the Finite Element Method (FEM) technique well suited for further research into these CAM states. However, before applying FEM to CAM states, one will need a review of the FEM technique.

\subsubsection{General Theoretical Basis}

A brief overview of the method should be sufficient to give an idea on how it works. The main difference between FDTD and FEM is that FDTD reduces the equations of motion to sum expressions that can be implemented on a computer and used in an time-iterative manner to obtain a solution. The FEM technique transforms the equations of motion to a matrix equation and uses matrix methods to obtain a solution. To solve eigenvalue problems, the FEM technique is preferable because solving eigenvalue problems in matrix forms requires one to diagonalize some matrix, which is equivalent to solving the eigenvalue problem. The diagonalization would be nontrivial but what is gained is a fine decomposition of the spectrum of solutions to the problem. 
The FEM technique requires two important pieces of information: the governing partial differential equation (PDE) in some domain $\Omega$,

$$
L \phi=f
$$

together with the boundary conditions on the boundary $\Gamma$ that encloses the domain $\Omega$. In $3.14, L$ is the differential operator, $f$ is the excitation or forcing functions, and $\phi$ is the unknown quantity. The goal of the FEM technique is to express 3.14 in a form applicable over simple sub-domain shapes that approximate the entire domain and then integrate all the results to obtain a solution.

It would be ideal to solve 3.14 over its boundary analytically, but this only happens for very simple problems. In real world problems, obtaining an analytical solution to complicated PDEs over intricate boundary conditions rarely happens. To overcome this, approximate methods have been developed. The two most popular methods in FEM are the Rayleigh-Ritz method and the Galerkin method. The RayleighRitz method is a variational method in which the boundary-value problem is formulated in terms of a variational expression called a functional. The minimum of this functional corresponds to a solution of the governing differential equation under the given boundary conditions. The approximate solution is then obtained by minimizing the functional with respect to parameters that define a certain trial solution. This particular method will not be discussed any further because the commercial software used in this thesis used the Galerkin method.

The Galerkin method is a weighted residual method, which seeks the solution by weighting the residual of the differential equation in an iterative manner. In $3.14, \phi$ is 
the exact solution. Assuming one does not know the exact solution, one assumes an approximate answer, $\widetilde{\phi}$, and formulates the residual,

$$
r=L \widetilde{\phi}-f \neq 0
$$

The best approximation for $\tilde{\phi}$ will be the one that reduces the residual $r$ to the least value at all the points of $\Omega$. The weighted residual method enforces the condition,

$$
R_{i}=\int_{\Omega} w_{i} r d \Omega=0
$$

where $R_{i}$ is the weighted residual integral and $w_{i}$ are the chosen weighting functions. These weighting functions are usually chosen to be the same as those used for the expansion of the approximate solution. The expansion of the approximate solution is given by,

$$
\tilde{\phi}=\sum_{j=1}^{N} c_{j} d v_{j}=\{c\}^{T}\{v\}=\{v\}^{T}\{c\}
$$

where the $v_{j}$ are the chosen expansion functions defined over the entire domain and $c_{j}$ are constant coefficients to be determined. In addition, \{\} denotes a column vector and the superscript $T$ denotes the transpose of the vector.

Selecting the weighting functions to be,

$$
w_{i}=v_{i} \quad i=1,2,3, \ldots, N
$$

so that 3.16 becomes,

$$
R_{i}=\int_{\Omega}\left(v_{i} L\{v\}^{T}\{c)-v_{i} f\right) d \Omega=0 \quad i=1,2,3, \ldots . N
$$

This can then be expressed as a matrix system, 


$$
[S]\{c\}=\{b\}
$$

where the elements of $[S]$ are given by

$$
S_{i j}=\frac{1}{2} \int_{\Omega}\left(v_{i} L v_{j}+v_{j} L v_{i}\right) d \Omega
$$

and the elements in $\{b\}$ are given by

$$
b_{i}=\int_{\Omega} v_{i} f d \Omega .
$$

It is very important that the right expansion functions be chosen to obtain an approximate answer that is close to the exact answer. For many problems this is very difficult if not impossible, and this is particularly true for two- and three- dimensional problems. To alleviate this difficulty, one can divide the entire domain into small subdomains and use trial functions defined over each subdomain. These trial functions are usually simpler than entire-domain functions since the subdomains are small, hence do not need to vary in a complicated manner to approximate the exact solution.

The discretization of the domain is important because it will affect the computer storage requirements, the computation time, and the accuracy of the numerical results. These subdomains are called elements and there are a finite number of them, hence the name: finite element. Figure 3.32 shows typical shapes for one-, two-, and threedimensional subdomains. 


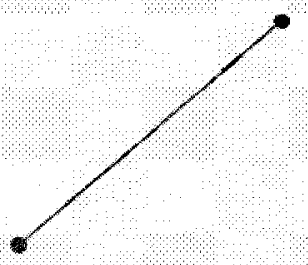

(a)

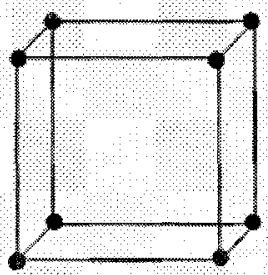

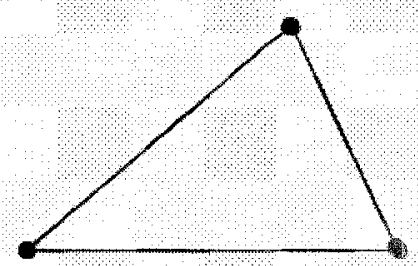

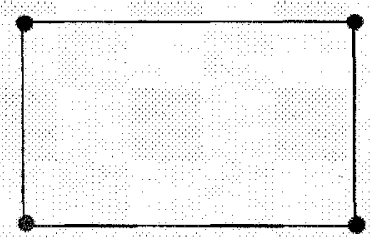

(b)
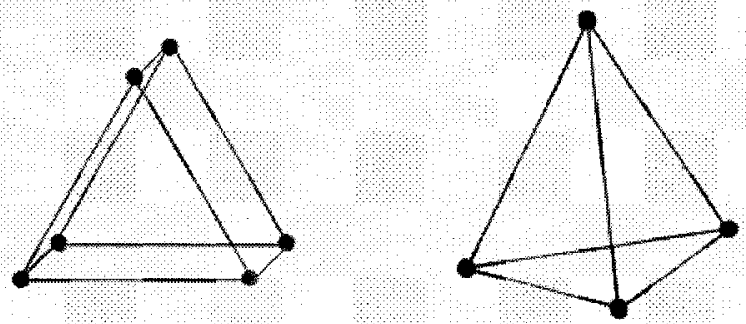

(c)

Figure 3.32. Basic finite elements. (a) One-dimensional. (b) Two-dimensional. (c) Threedimensional.

After the domain had been discretized, the selection of the basis functions is required to provide the approximation of the unknown solution within an element. Typically, these basis functions are selected to be polynomials of first (linear), second (quadratic), or of a higher order. On a side note, using polynomials to approximate functions is a fundamental process in mathematical physics, because the Dirac function can be approximated by a polynomial [49]. Once the order of the polynomial is selected, one derives an expression for an unknown solution in an element, in the following form:

$$
\widetilde{\phi}^{e}=\sum_{j=1}^{N} N_{j}^{e} \phi_{j}^{e}=\left\{N^{e}\right\}^{T}\left\{\phi^{e}\right\}=\left\{\phi^{e}\right\}^{T}\left\{N^{e}\right\}
$$

where $n$ is the number of nodes in the element, $\phi_{j}^{e}$ is the value of $\phi$ at node $j$ of the element $e$, and $N_{j}^{e}$ is the basis function for node $j$. 
After selecting the element basis functions, the system of equations must be formulated. These are where the matrix equations are obtained. For 3.14, the weighted residual for the $e$ th element is,

$$
R_{i}^{\prime}=\int_{\sigma^{\circ}} N_{i}^{e}\left(L \widetilde{\phi}^{e}-f\right) d \Omega \quad i=1,2,3, \ldots, n
$$

Substituting 3.23 in 3.24 yields,

$$
\left.R_{i}^{*}=\int_{\Omega^{*}} N_{i}^{e} L\left\{N^{e}\right\} d \Omega \phi^{e}\right\}-\int_{\Omega^{*}} N_{i}^{*} d \Omega \quad i=1,2,3, \ldots, n
$$

which can be written in matrix form as

$$
\left\{R^{e}\right\}=\left[K^{e}\right\}\left\{\phi^{e}\right\}-\left\{b^{e}\right\}
$$

Summing over all elements,

$$
\{R\}=\sum_{e=1}^{M}\left\{R^{e}\right\}=\sum_{e=1}^{M}\left(\left[\bar{K}^{e}\right]\left\{\bar{\phi}^{e}\right\}-\left\{\bar{b}^{e}\right\}\right)
$$

where the overbar denotes an expansion or augmentation between local and global node numbers. Local nodes describe internal element indices and global nodes describe nodes in relation to the entire domain. The system of equations can then be obtained by setting 3.27 to zero, resulting in,

$$
\sum_{e=1}^{M}\left(\left[\bar{K}^{e}\right]\left\{\bar{\phi}^{e}\right\}-\left\{\bar{b}^{e}\right\}\right)=\{0\}
$$

Before the system of equations is ready to solve for a specific solution, one needs to apply the required boundary conditions. In some cases the boundary condition must be applied explicitly (i.e. Dirichlet boundary conditions) while others are usually satisfied implicitly and automatically in the solution process (i.e. Neumann boundary conditions). 
Solving the system of equations is now the concluding step in the FEM process. The resultant system has one of the following two forms:

$$
[K]\{\phi\}=\{b\}
$$

or

$$
[A]\{\phi\}=\lambda[B]\{\phi\}
$$

Equation 3.29 is of the deterministic type that is used, for example, in harmonic propagation, while 3.30 is the eigenvalue type. Both types will be used in this thesis for the study of PhQ modes. In order to obtain 3.30 from 3.29 , the known vector $\{b\}$ vanishes and the matrix $[K]$ can be written as $[A]-\lambda[B]$, where $\lambda$ is an eigenvalue.

The actual implementation of the FEM technique was done using the commercially available software tool COMSOL®. Before using this software to further study CAM states in PhQs, one needs to verify that the software yields similar results to data already accepted in the literature.

\subsubsection{Modeling PhC Bandstructures as Verification}

In order to show that COMSOL® can be used as a reliable analysis tool, the bandstructures of various PhCs were found. The primitive cell of square, vein, and hexagonal PhCs were drawn, as shown in figure 3.33, with their respective primitive cell vectors. After specifying the high and low dielectric regions and periodic boundary conditions, an eigenmode analysis to find a number of eigenmodes was completed. The lowest order modes are shown in figure 3.34. 

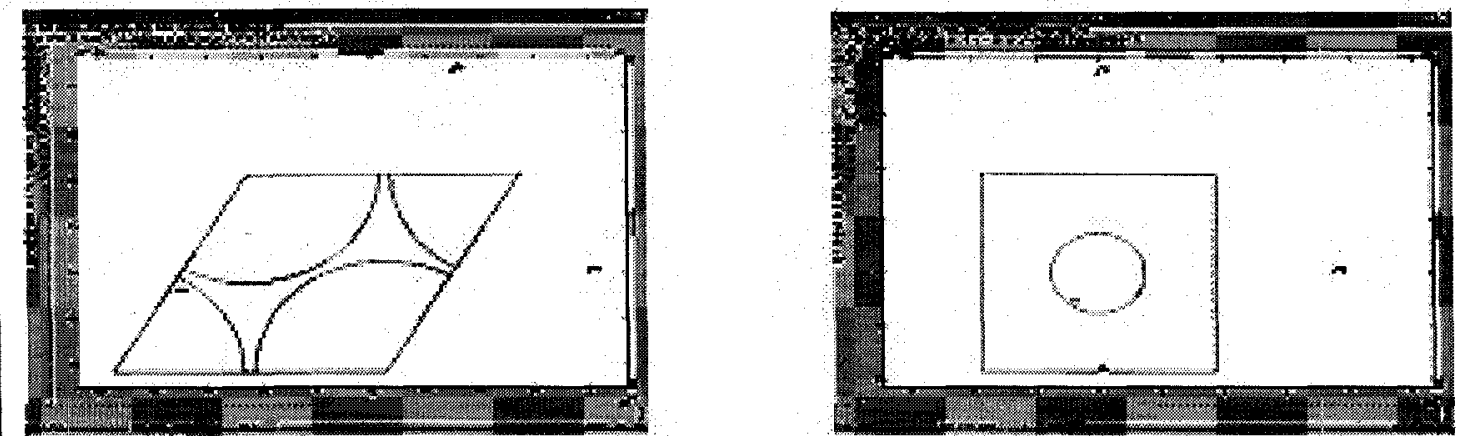

$(9)$

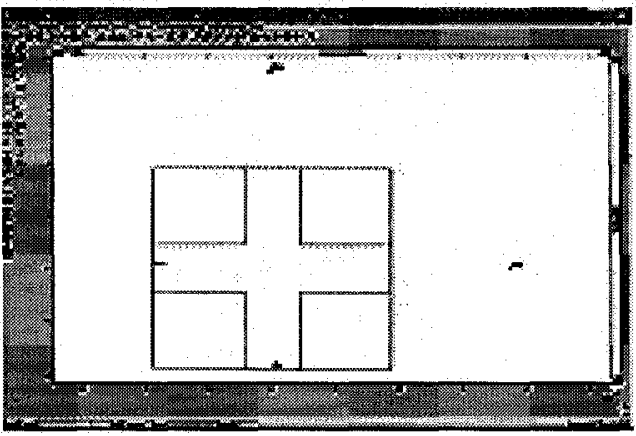

(b)

(Q)

Figure 3.33. COMSOL screen captures are setting up primitive cells of (a) a hexagonal structure, (b) square structure and (c) vein structure.

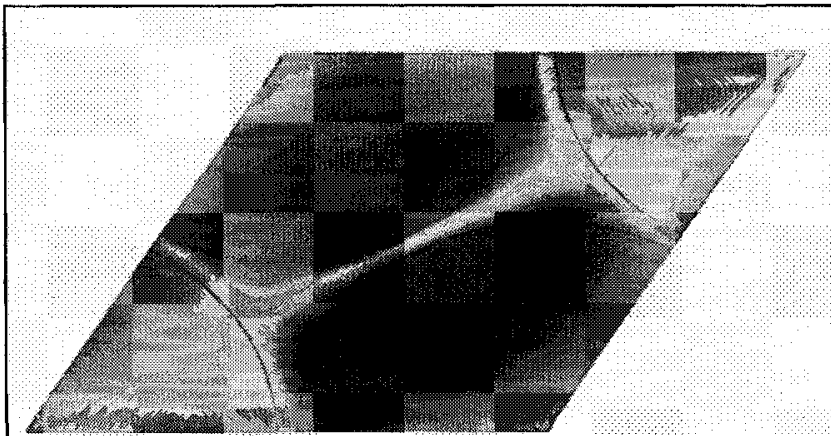

(a)

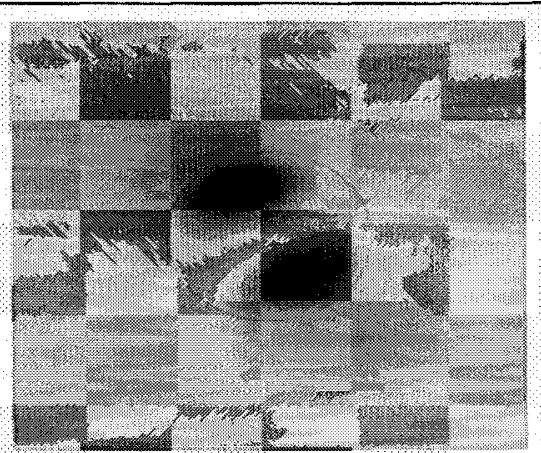

(b)

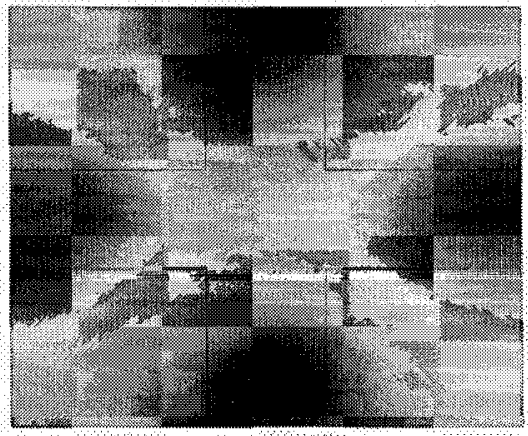

(c)

Figure 3.34. Lowest order field modes of eigenvalue problem of (a) a hexagonal structure, (b) square structure and (c) vein structure. 
COMSOL® takes each session and builds a FEM structure, which can then be exported to its script program called COMSOL Script ${ }^{\circledR}$. This tool is very similar to MATLABß. A script code can be written to run subsequent FEM analysis with the parameters slightly changed. The parameter that changed was the wavevector at the periodic boundary. It was iterated along the associated Brillouin zone of the primitive cell of each lattice type. At each wavevector iteration, a few eigenmodes were obtained. Once the iteration was complete, bandstructure diagrams were then be compared to published results, as shown in figure 3.35.

An approximate bandstructure of a PhQ was obtained by forcing a hexagonal primitive cell on the PhQ, as shown in 2.14 , similar to the way a planewave analysis was done in Chapter 2. In this case, it was a finite element analysis. The result is shown in figure 3.36 , and when one compares it to figure 2.15 , it shows close agreement to the lower portion of the bandstructure.

All in all, the COMSOLB tool is producing results that are equivalent to those that are already published and one can rest assured that the results are indeed reliable. While this is the case, one must be aware that spurious answers do come up in FEM techniques, although these spurious results are only connected to curl equations in $3 \mathrm{D}$ [50]. The work in analyzing CAM states in PhQs is exclusively 2D, therefore spurious results are not of concern here. 


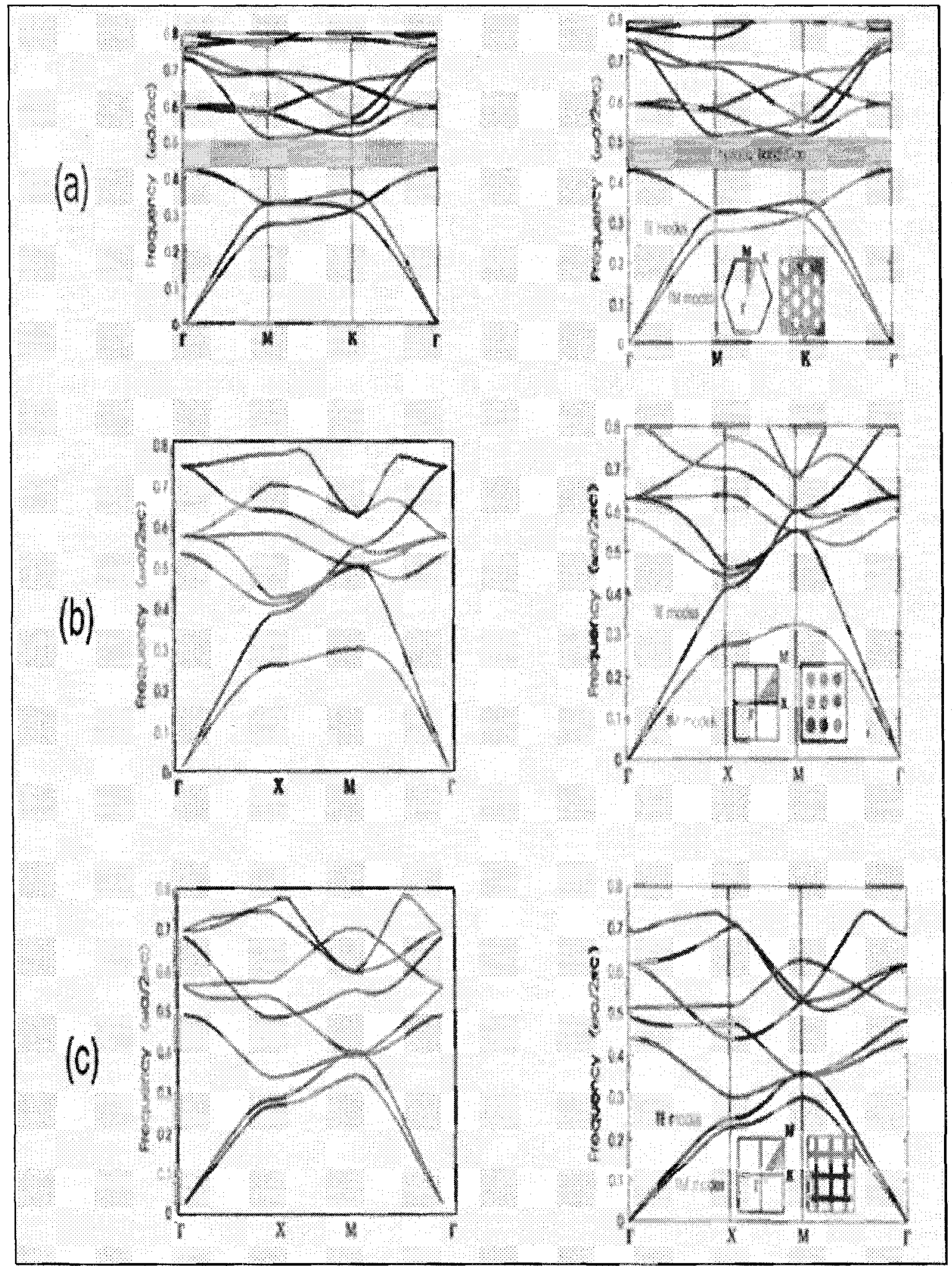

Figure 3.35. Comparison between simulated results (right) and published results [51] (left). (a) hexagonal structure, (b) square structure and (c) vein structure. 


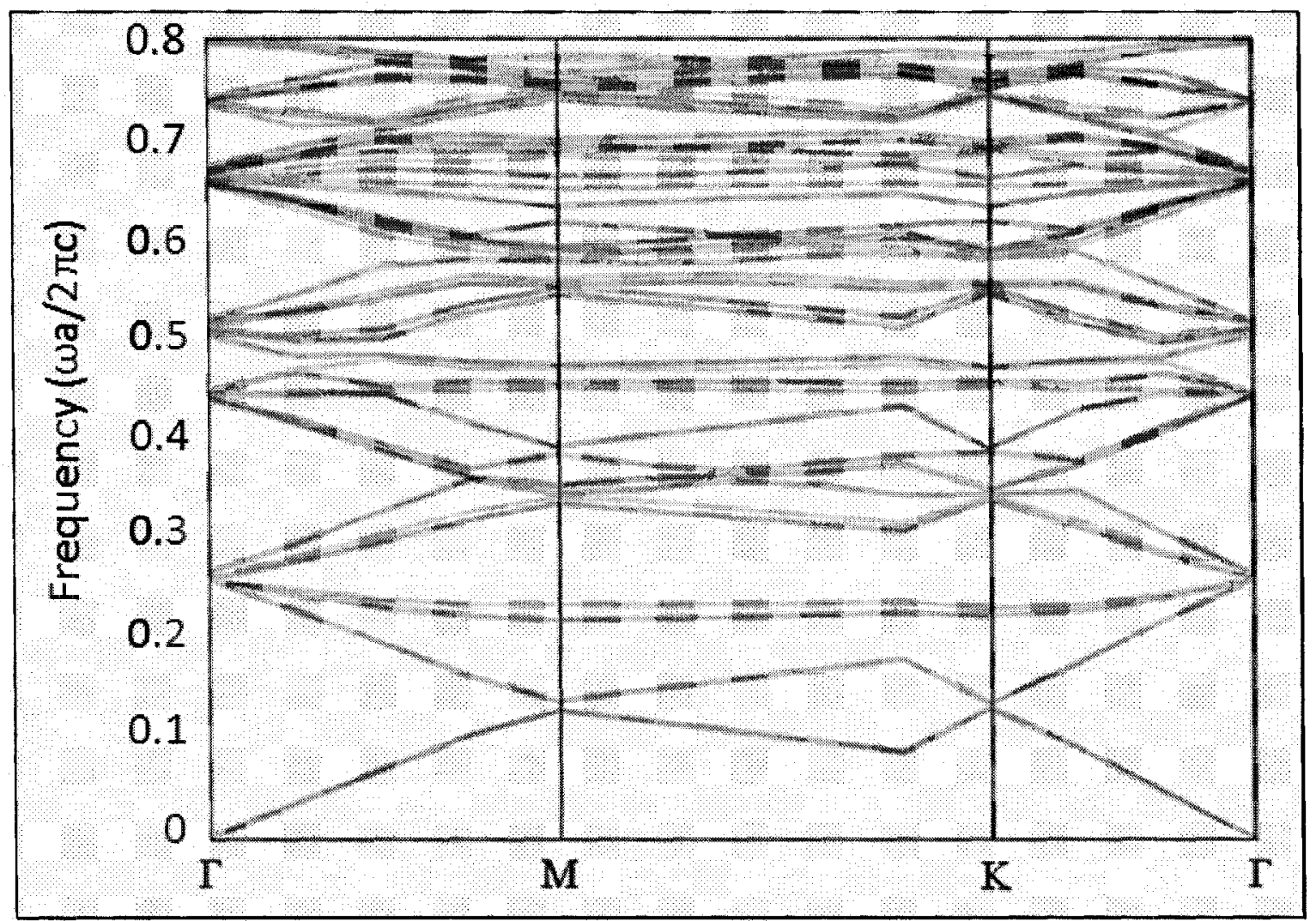

Figure 3.36. Approximate bandstructure of PhQ using FEM technique.

\subsubsection{Importing PhQ Patterns for FEM Analysis}

The next step after software verification is importing the structure to simulate. Professor Gauthier's Beam@ software could generate and output the files in almost any format but it required writing code to do that. The structures of figure 3.5, figure 3.7, and figure 3.8 were all generated using this software. At the same time, Mathematica ${ }^{\circledR}$ was used to also generate PhQ structures. It was important to generate these structures in such a way that the images were solely in black and white. This was done to make sure the contrast between high dielectric and low dielectric was clear. At the time of this research, importing geometries to simulate was not widely available, and if anything was going to be imported it would be a file format from AutoCad®. COMSOL® was able to import data exchanged files (DXFs). However, if the imported geometry was complicated, like 
the case with PhQs, then this process took a long time because the COMSOL(1) software had to fragment the pattern into polygons, as shown in figure 3.37.

To retain the proper curvatures, the polygons needed to be smaller. After the $2 \mathrm{D}$ PhQ structure was generated in Mathematica®, it was imported to another software, known as LinkCad® that transcoded the image format (usually JPEG) to DXF. From there, the DXF was then imported to COMSOL®. Because the PhQ in this study has a $20 \%$ fill factor, it made more sense to mesh the posts rather than mesh empty space. So the PhQ structure of figure 3.37 was inverted, as shown in figure 3.38. However, this was still computationally expensive because the software treated each line of the polygon with an approximate basis function. By converting the polygons to circles and ellipses, as shown in figure 3.39, the software could use other basis functions to improve computational efficiencies. 




Figure 3.37. (a) Importing DXF image of PhQ, (b) shows the level of polygons required to get curvature, and (c) is a close up of the polygons.

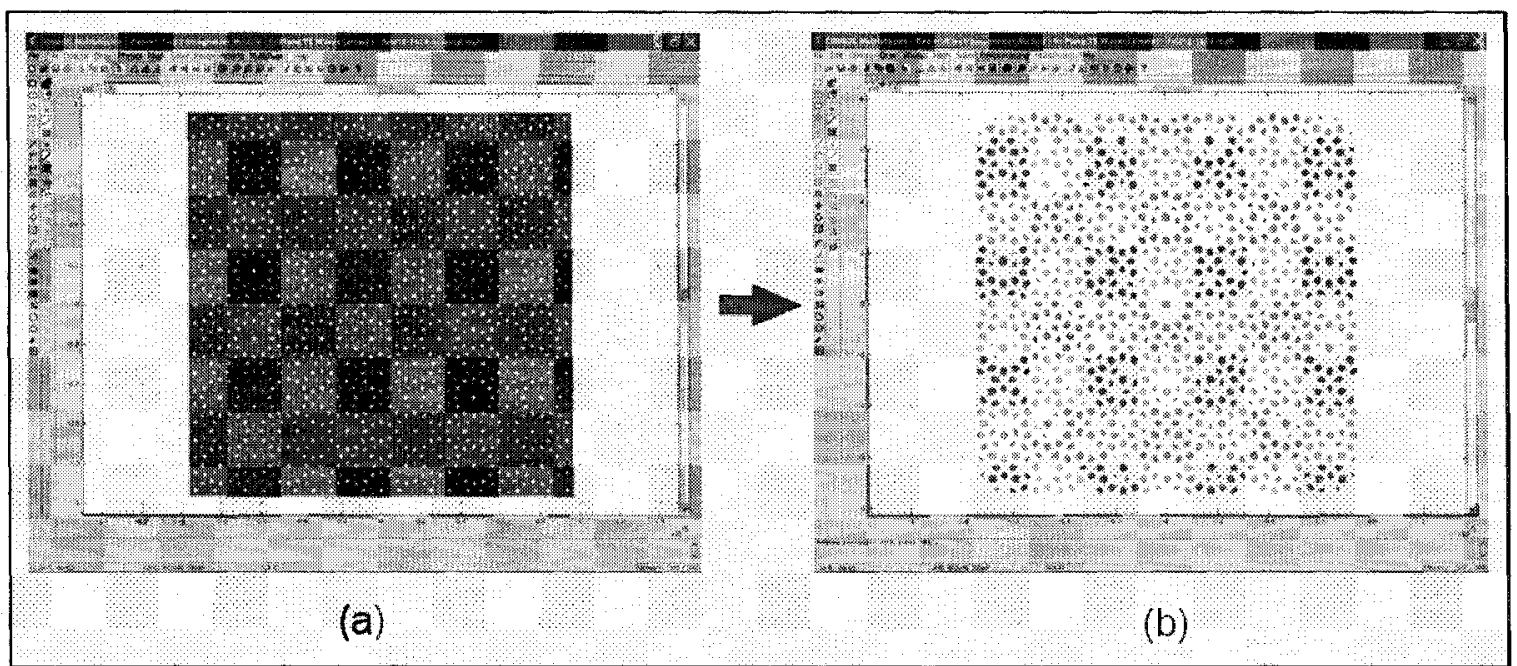

Figure 3.38. Inversion of PhQ structure so that meshing will occur over a smaller area which will save computation cycles. (a) imported structure and (b) inverted structure. 


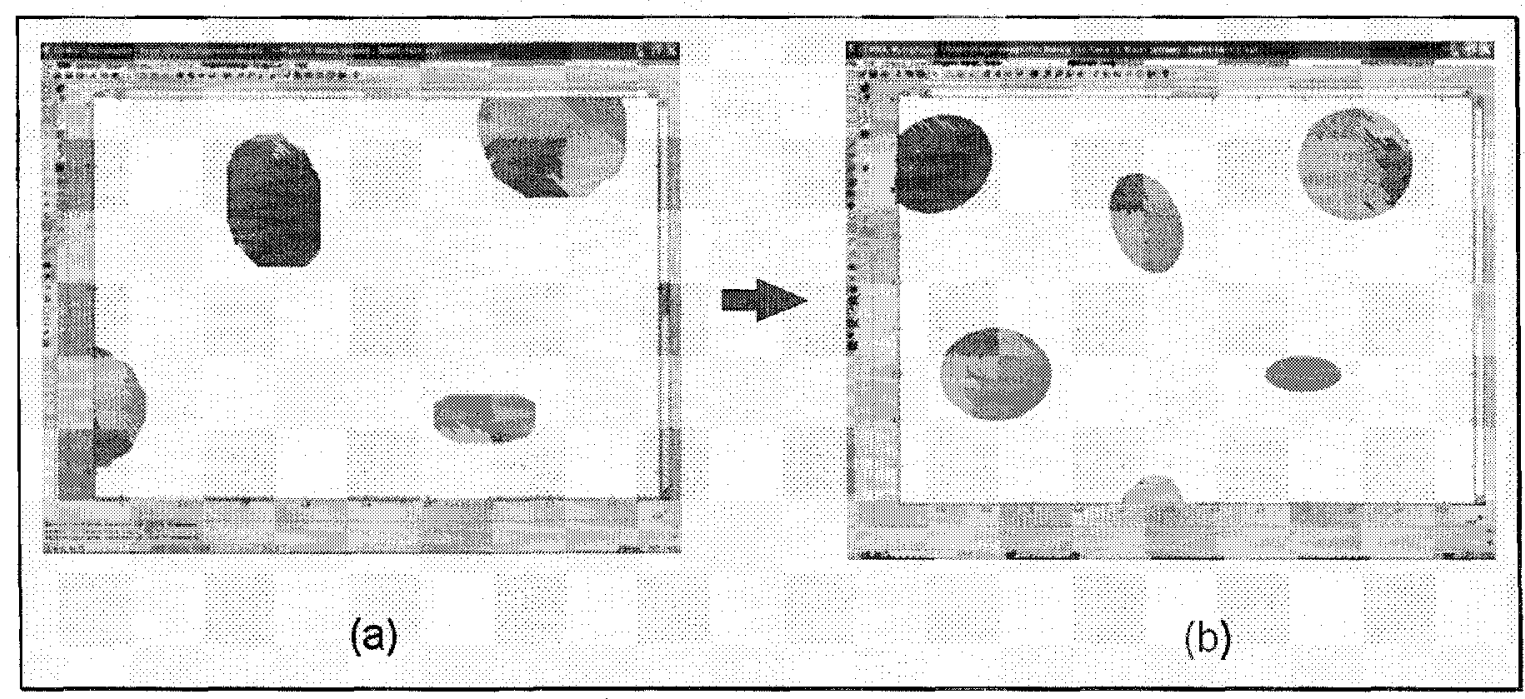

Figure 3.39. Converting polygons into circles and ellipses.

\subsubsection{FEM Eigenvalue Analysis of $P h Q s$}

Now that the PhQ pattern can be imported into COMSOL®, and a mesh can be applied to the pattern as shown in figure 3.40 , one can now run an eigenvalue analysis on the $\mathrm{PhQ}$ pattern. The results of an initial eigenvalue analysis showed a range of central localized modes that had the symmetry of the PhQ. Figure 3.41 shows two examples of the modes found. As the analysis proceeded further, more centralized modes were found with other rotational symmetries. They resembled cavity modes that look like spherical harmonic functions without an azimuthal part. One can represent the modes of figure 3.41 as the top view of a spherical domain that contains the spherical harmonics, as shown in figure 3.44. One also notices this for defect states in regular PhCs. Figure 3.43 shows defect states for a square array $\mathrm{PhC}$ (top portion) and a hexagonal array $\mathrm{PhC}$ (bottom portion). One can also view these as a mixture of 4-fold and 6-fold quasicrystals that have translational periodicity.

With these results, the postulate of section 3.2.8 that predicts the existence of $2 l+1$ states of an $l$-fold PhQ can now be tested. 


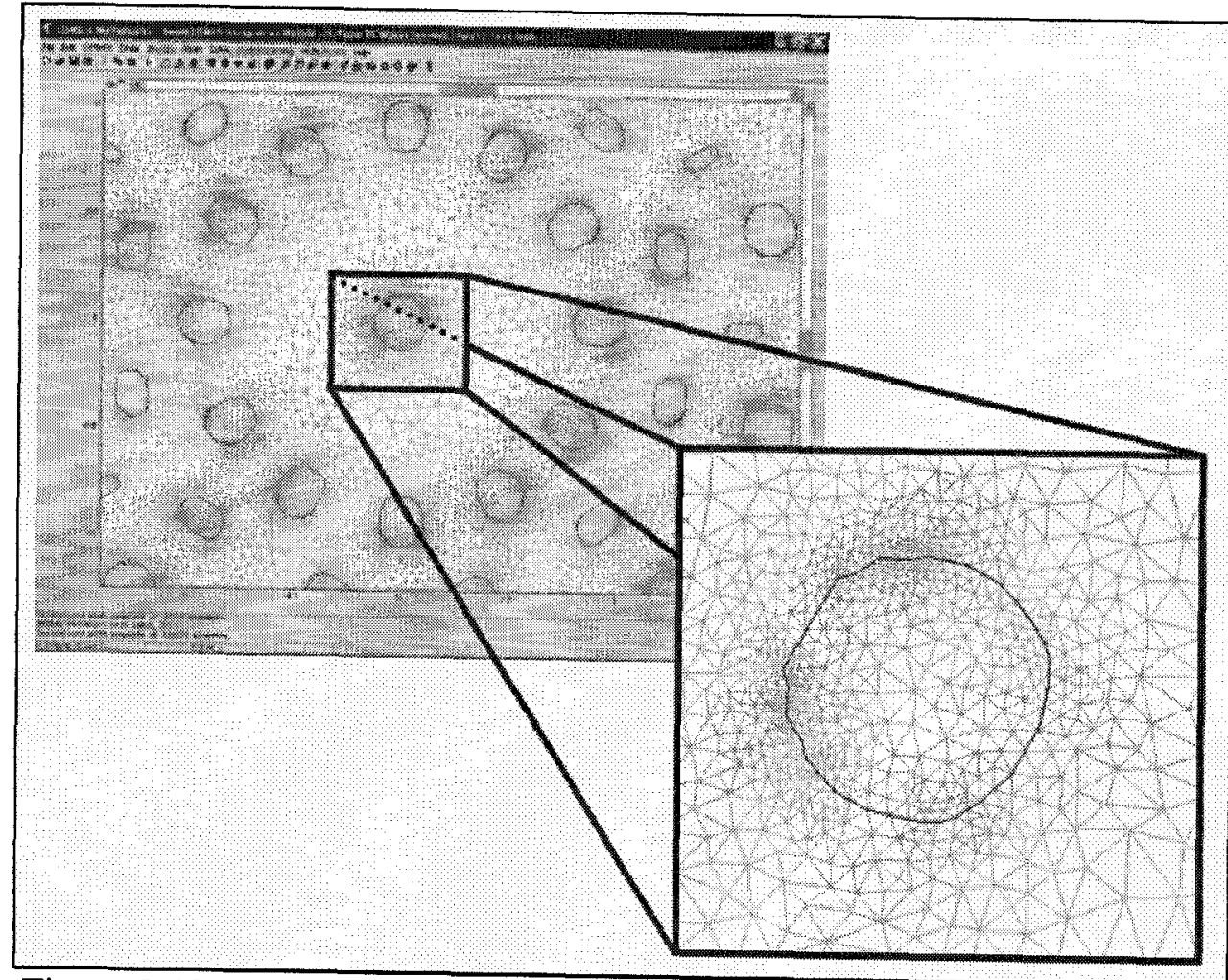

Figure 3.40. Finite element mesh of imported PhQ pattern.

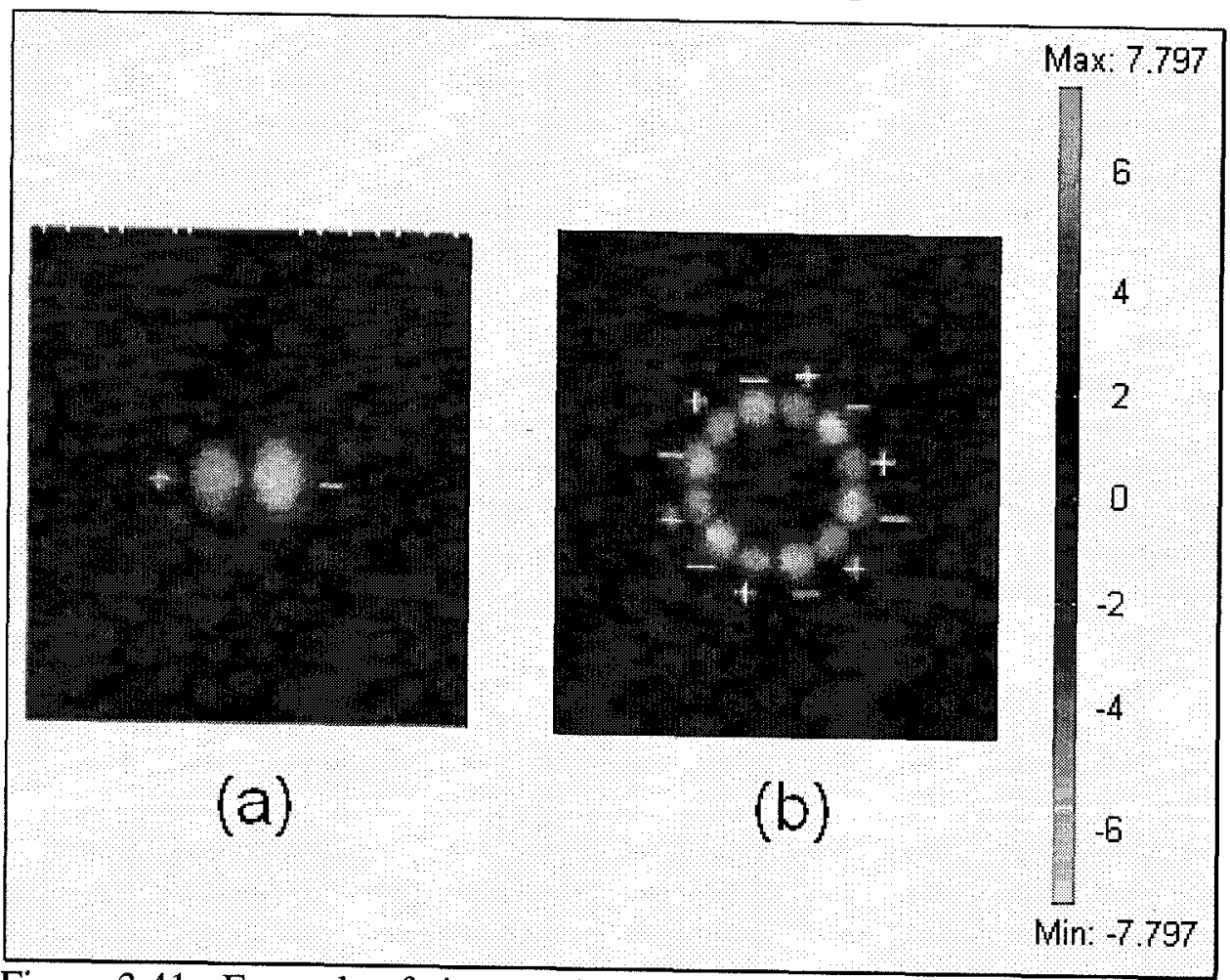

Figure 3.41. Example of eigenmodes found in FEM analysis. (a) appears to be a dipole mode and (b) appears to have 12 lobes but with half of the opposite polarity. 


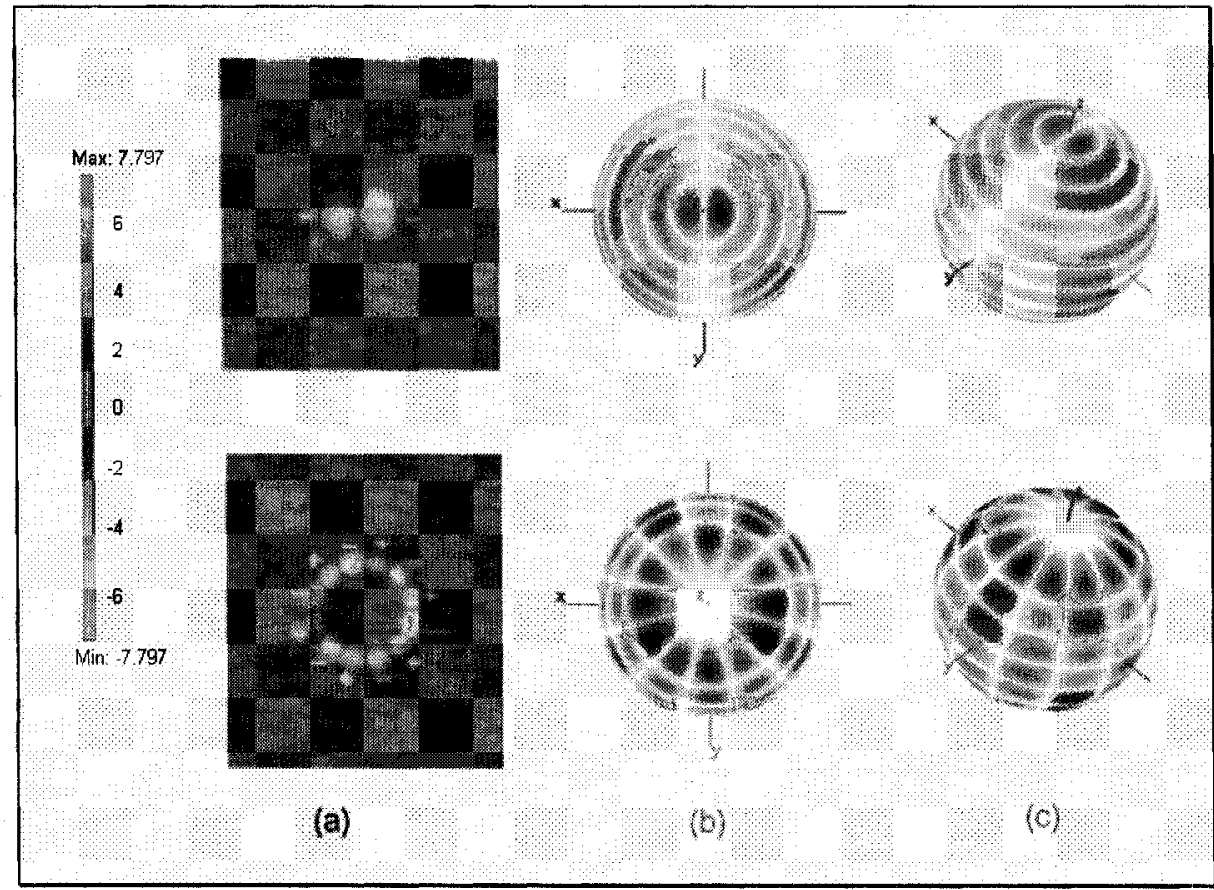

Figure 3.42. Resemblance between eigenmodes and spherical harmonics. (a) Eigenmodes, (b) top of sphere domain which harmonic functions are solved on, and (c) isometric view of sphere domain. Color legend of 3.41 applies for (a).

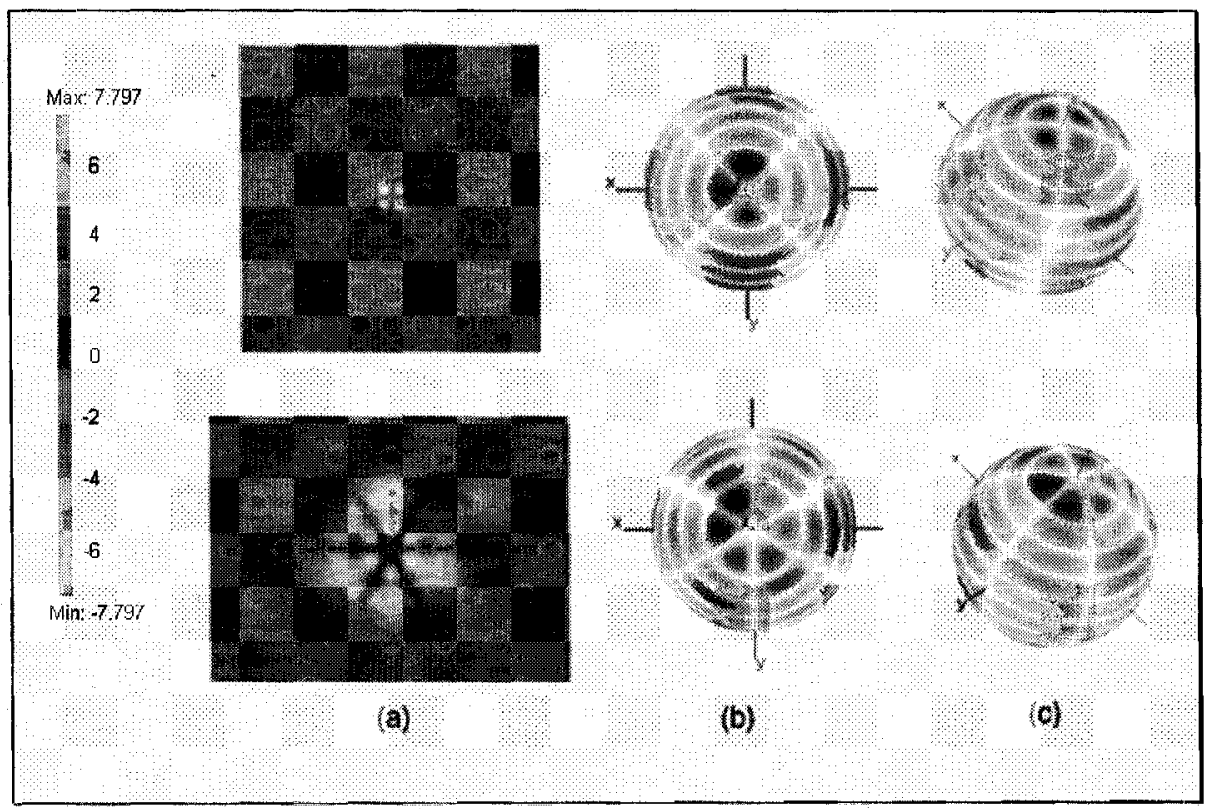

Figure 3.43. Resemblance between eigenmodes and spherical harmonics of square array $\mathrm{PhC}$ (top) and hexagonal array $\mathrm{PhC}$ (bottom). (a) Eigenmodes, (b) top of sphere domain which harmonic functions are solved on, and (c) isometric view of sphere domain. One can also view these as a mixture of 4-fold and 6-fold quasicrystals that have translational periodicity. Color legend of 3.41 applies for (a). 


\subsection{CAM States of PhQs}

The postulate of section 3.2.8 states that the defect-free localized modes in PhQs results from the rotational symmetry of the quasicrystal lattice and are equivalent to Bloch modes of translational systems. The rotational foldness of the lattice at some point is equivalent to an orbital angular momentum number, $l$, which gives rise to $2 l+1$ defectfree localized modes about that point. The DFF of the lattice makes the existence of these modes possible. It turns out that the DFF of the 12 -fold $20 \%$ fill factor PhQ facilitates the emergence of these modes. Figure 3.44 shows these novel results [10].

Figure 3.44 shows thirteen modes labeled from $m=0$ to $m=12$. This labeling is reminiscent of quantum angular momentum numbers. The $m$ number counts half the number of lobes in the modes that are just inside the inner ring of 12 rods. Also, under each mode image is the eigenvalue wavelength in which these modes occur.

Figure 3.44 can be represented mathematically as,

$$
\psi(\mathbf{r}) \equiv\langle\mathbf{r} \mid \psi\rangle=\sum_{l, m}\langle\mathbf{r} \mid l, 0\rangle\left\langle l, 0\left|R_{z}(\phi)\right| l, m\right\rangle\langle l, m \mid \psi\rangle
$$

where $\psi(\mathbf{r})$ is the scalar transverse electric field, $\langle\mathbf{r} \mid \psi\rangle$ is the Dirac notation of this field quantity, $\langle l, m \mid \psi\rangle$ is the component projecting the field value on the angular momentum modes, $\left\langle l, 0 \mid R_{z}(\phi) l, m\right\rangle$ are the spherical harmonic functions in Dirac notation, and $\langle\mathbf{r} \mid l, 0\rangle$ is the radial dependence term that is directly related to the DFF. Being able to show that all the spherical harmonic modes for 12-fold PhQ are present depends upon the DFF. It is this reason why Chen et al. [8] found localized modes for the octagonal PhQ structure while Wang et al. [9] did not find any modes for his octagonal PhQ structure. 


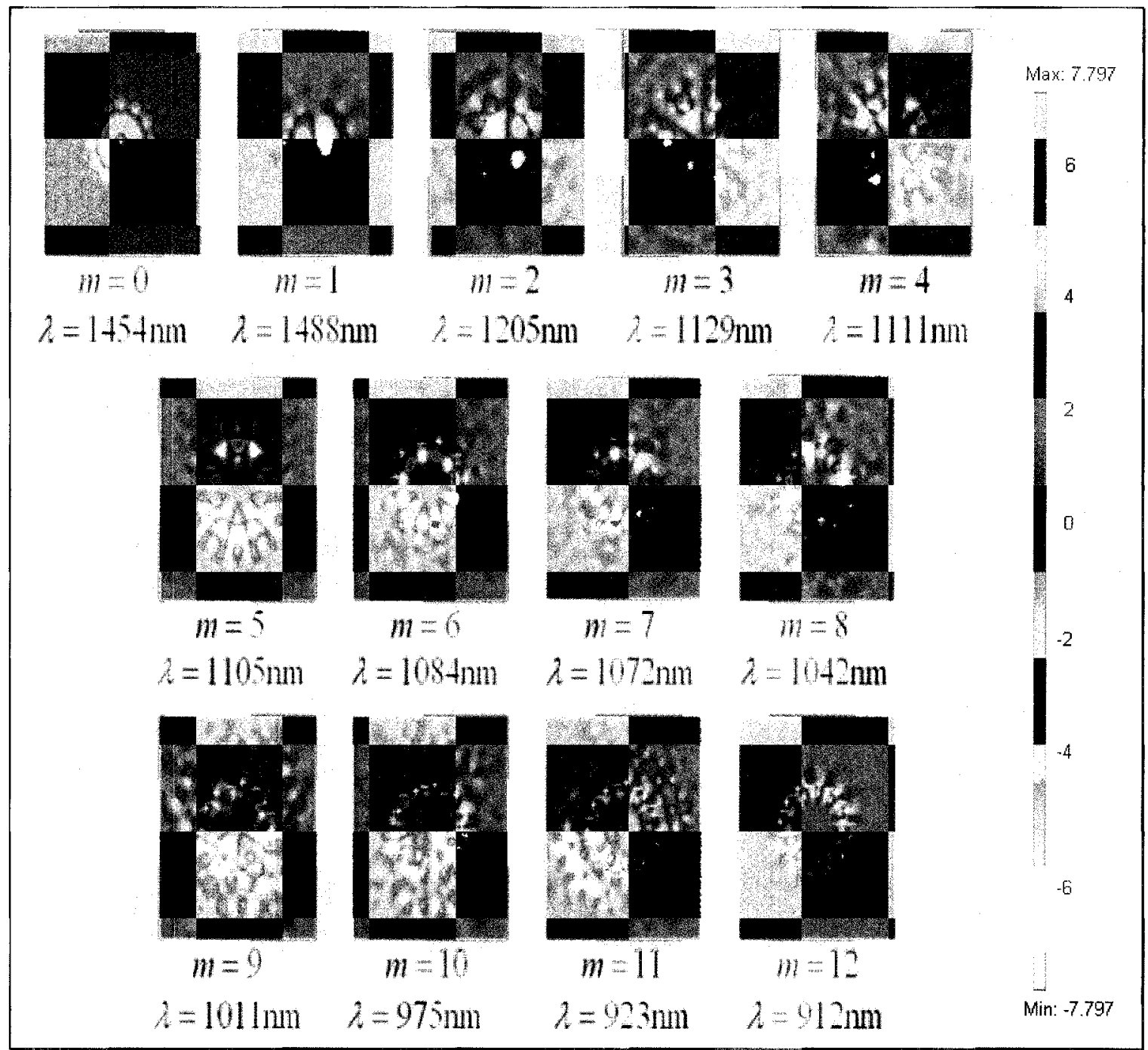

Figure 3.44. Localized optical modes in a 12-fold $\mathrm{PhQ}$. The number of negative lobes (represented in red and a negative sign) or the number of positive lobes (represented in blue and a positive sign) equals the $m$ number. The wavelength at which these modes occurred are shown underneath each respective mode [10].

The question remains: why should one call these states crystal angular momentum states? There are three novel and compelling reasons to do so:

(1) localized modes of a $2 \mathrm{D}$ PhQ pattern are equivalent to spherical harmonics on a 3D sphere via a Riemann sphere transformation,

(2) the DFF violates group theory predication of eigenmodes, and

(3) an optical Lamb shift is observed between the $m=0$ and $m=1$ modes.

The subsequent three sections explain further these three points. 


\subsubsection{Riemann Sphere Transformation}

Spherical harmonics are functions defined on a 3D sphere. They are used in atomic physics to describe the angular dependence of the wave function of an electron around a central potential.

The problem centers on how can one associate spherical harmonics, which are of a $3 \mathrm{D}$ character with $2 \mathrm{D} \mathrm{PhQs}$, which are of a $2 \mathrm{D}$ character? There is a mathematical construct, known as the Riemann sphere, that maps a $2 \mathrm{D}$ infinite plane onto a $3 \mathrm{D}$ sphere. The entire PhQ plane, which includes infinity, can be transferred to a $3 \mathrm{D}$ sphere and the spherical harmonics can be overlaid on this pattern. This mapping is called a stereographic projection. Figure 3.45 shows this process.

For now, this transformation is only a heuristic tool to arrive at a link between the rotational symmetry of the $\mathrm{PhQ}$, spherical harmonics, and angular momentum. Currently, a fully rigorous mathematical description is still unknown. Nonetheless, this construct was initially conceived of first before the results of figure 3.44 were obtained. and was a motivating concept for the search of all of the CAM states in figure 3.44. Figure 3.46 is a reinterpretation of the $m=1$ state in figure 3.42 . 


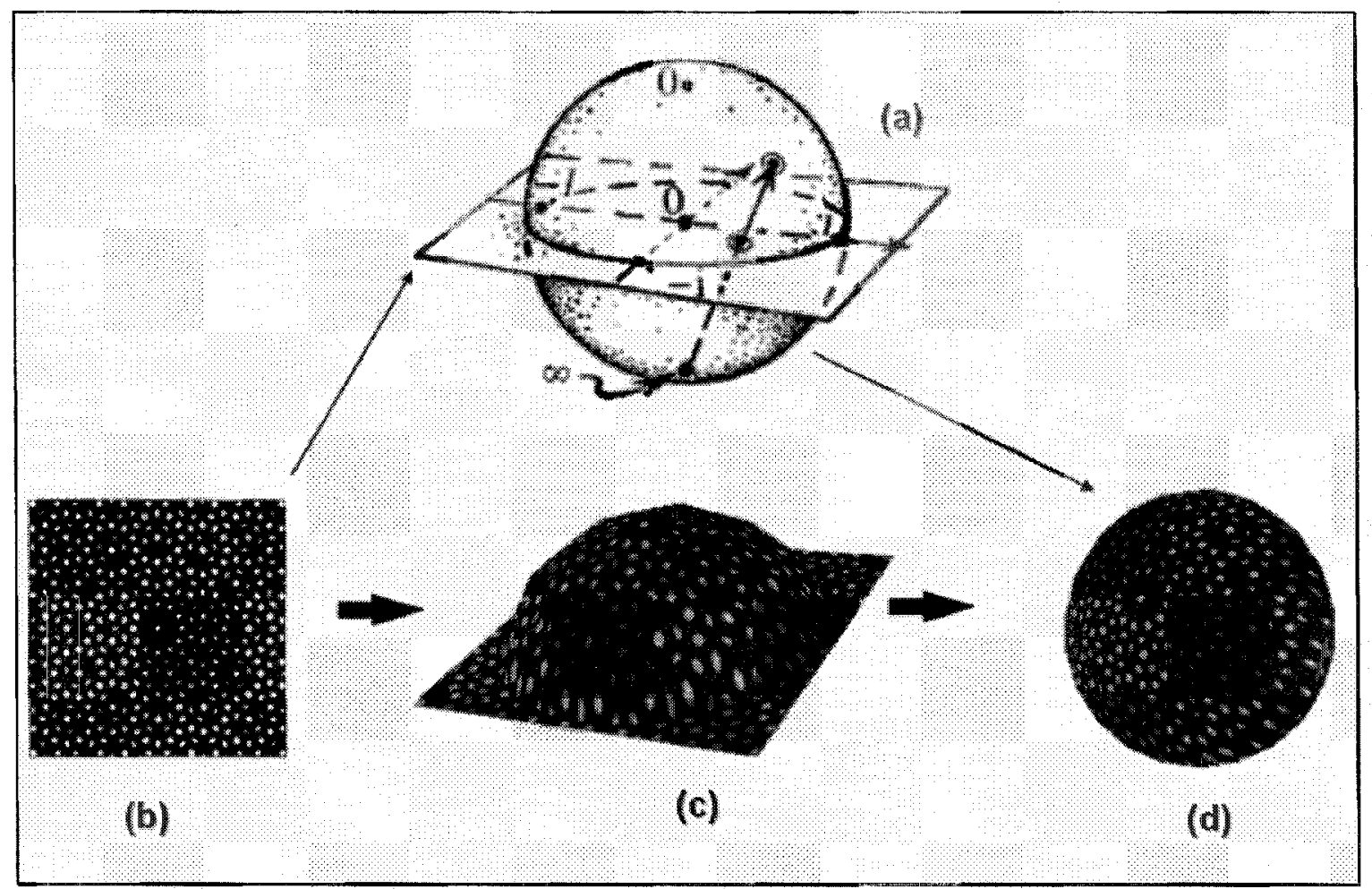

Figure 3.45. Stereographic projection of 2D PhQ pattern to 3D sphere. (a) Riemann sphere definition. (b) 2D PhQ pattern, (c) 2D to 3D transformation, (d) 3D projection of 2D sphere.

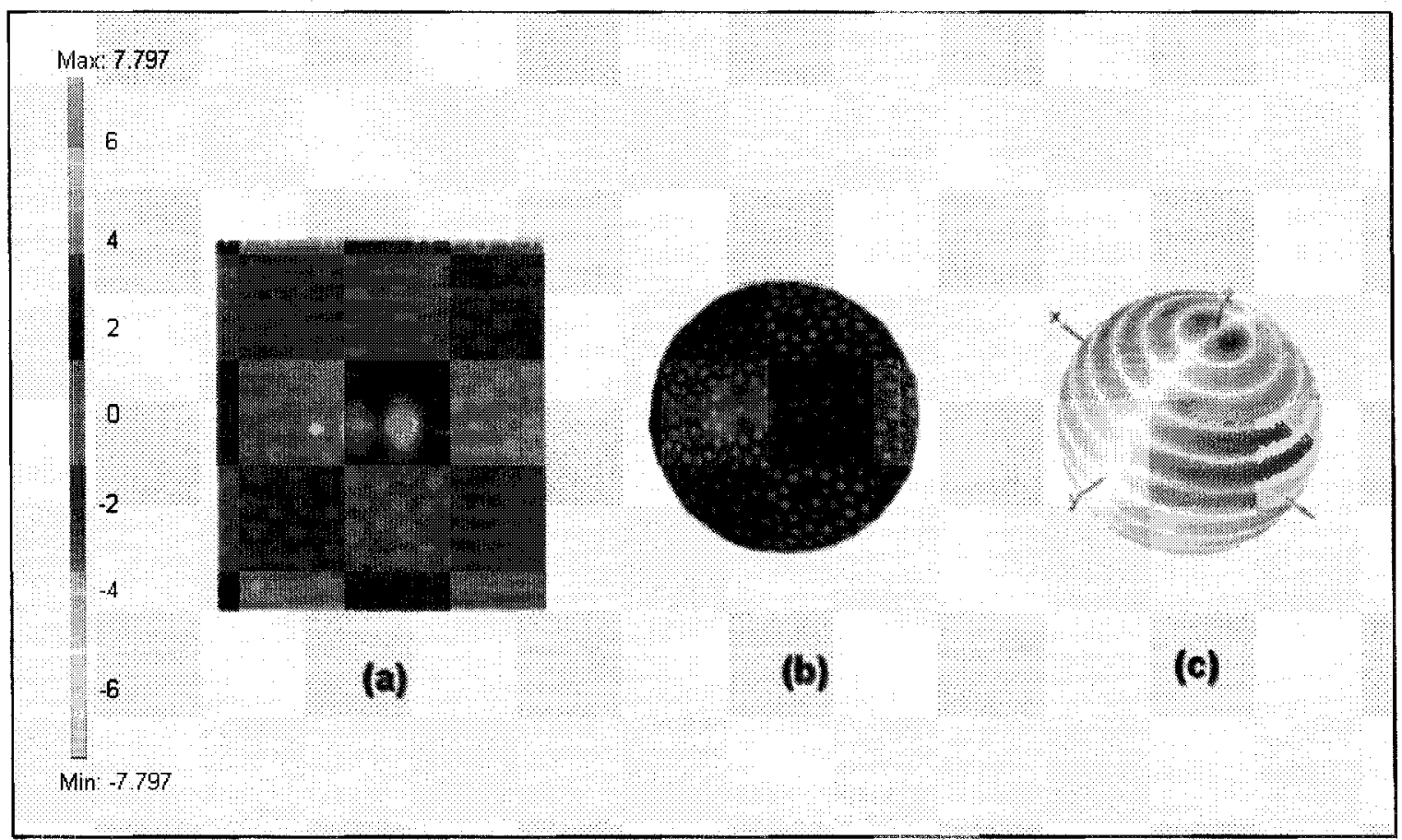

Figure 3.46. (a) PhQ CAM $m=1$ state, (b) Riemann sphere map of state, (c) equivalent spherical harmonic mode. 


\subsubsection{Group Theory Predications}

If the DFF in the 12-fold PhQ was made from point scatterers, one can apply a point group about the center point of the pattern. This symmetry group, $C_{12 v}$, consists of nine classes of conjugate elements,

$$
C_{12 v}=\left\{E, 2 C_{12}, 2 C_{6}, 2 C_{4}, 2 C_{3}, 2 C_{12}^{5}, C_{2}, 6 \sigma_{x}, 6 \sigma_{d}\right\}
$$

Due to $C_{12 v}$ having 9 classes of conjugate elements, group theory states that the number of irreducible representations is equal to the number of classes of conjugate elements. Group theory also states that the number of irreducible representations is equal to the number of eigenmodes. In this case, group theory says that this 12-fold PhQ should have 9 modes:

(1) two nondegenerate $m=0$ modes, known as $A_{1}$ and $A_{2}$,

(2) two nondegenerate $m=6$ modes, known as $B_{1}$ and $B_{2}$,

(3) five doubly degenerate modes, $E_{1} \equiv(m=1), \quad E_{2} \equiv(m=2), \quad E_{3} \equiv(m=3)$,

$$
E_{4} \equiv(m=4), E_{5} \equiv(m=5) \text {. }
$$

This leaves out the $m=7$ to $m=12$ modes. However, when the scatterers have unconventional shapes, these modes can then be obtained. The DFF has selected out modes forbidden by group theory but allowed by the spherical harmonics. The author performed a group theory analysis of 12 -fold PhQs has discovered one more mode, in addition to the 25 modes from the $2 l+1$ rule. This extra mode is one of the two nondegenerate modes of the $m=0$, as figure 3.47 shows. 


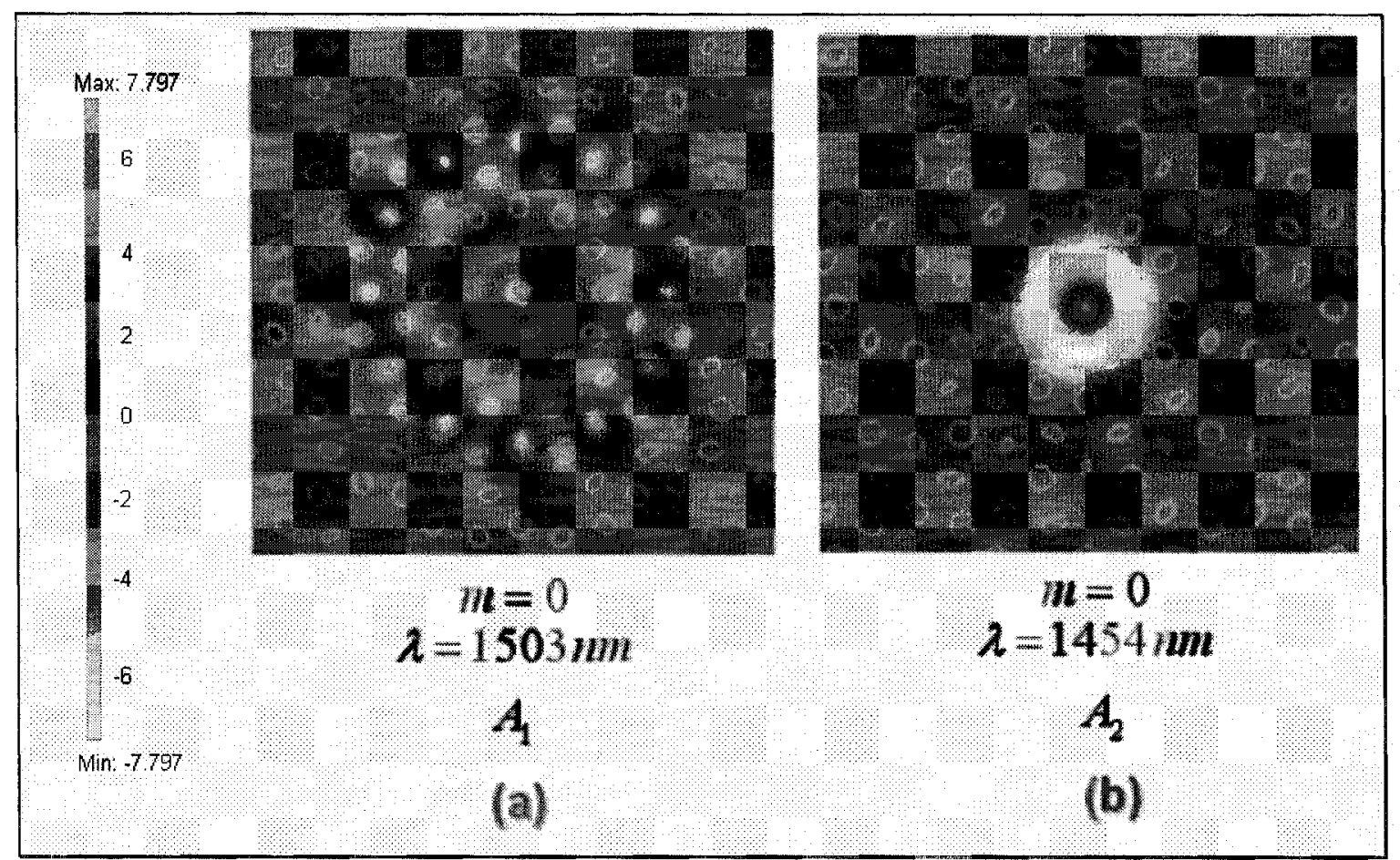

Figure 3.47. Group theory predicated $m=0$ modes.

\subsubsection{Optical Lamb Shift}

One of the most compelling arguments for crystal angular momentum is the value of the wavelengths for the $m=0$ mode and the $m=1$ mode in figure 3.44 . The $m=1$ mode has a larger wavelength than the $m=0$ mode and remaining modes combined. If the wavelength is larger, the associated energy of the mode is lower. In atomic systems, it has been found that the $p$-orbital has a lower energy then the $s$-orbital, known as the Lamb shift. This is due to quantum electrodynamic corrections generating from the Heisenberg uncertainties of the vacuum. In quantum field theories (QFTs), the vacuum is an active player in particle dynamics via virtual relations. The vacuum, in fact, can be polarized: it acts as a dielectric. Due to this interaction, the $p$-orbital has a lower energy than the $s$-orbital. The $p$-orbital has an angular momentum of $m=1$, and the $s$-orbital has an angular momentum of $m=0$. There are no QFT equations in the Maxwell equations 
used by COMSOL® however, this shifting result is present. It is believed that the only conclusion for these Lamb-like shifts in optical states is due to the DFF.

\subsubsection{CAM States of Different PhQ Foldness}

Wang et al. [9] could not find defect-less localized modes in octagonal or decagonal PhQs. However, their DFFs were made from circular scatterers. In this thesis, octagonal and decagonal PhQs with non-circular DFFs are able to generate defect-less localized modes, as shown in figure 3.48 and figure 3.49. These structures are rod-type configurations. It should be noted that this work is the first to show defect-less localized modes for decagonal structures.

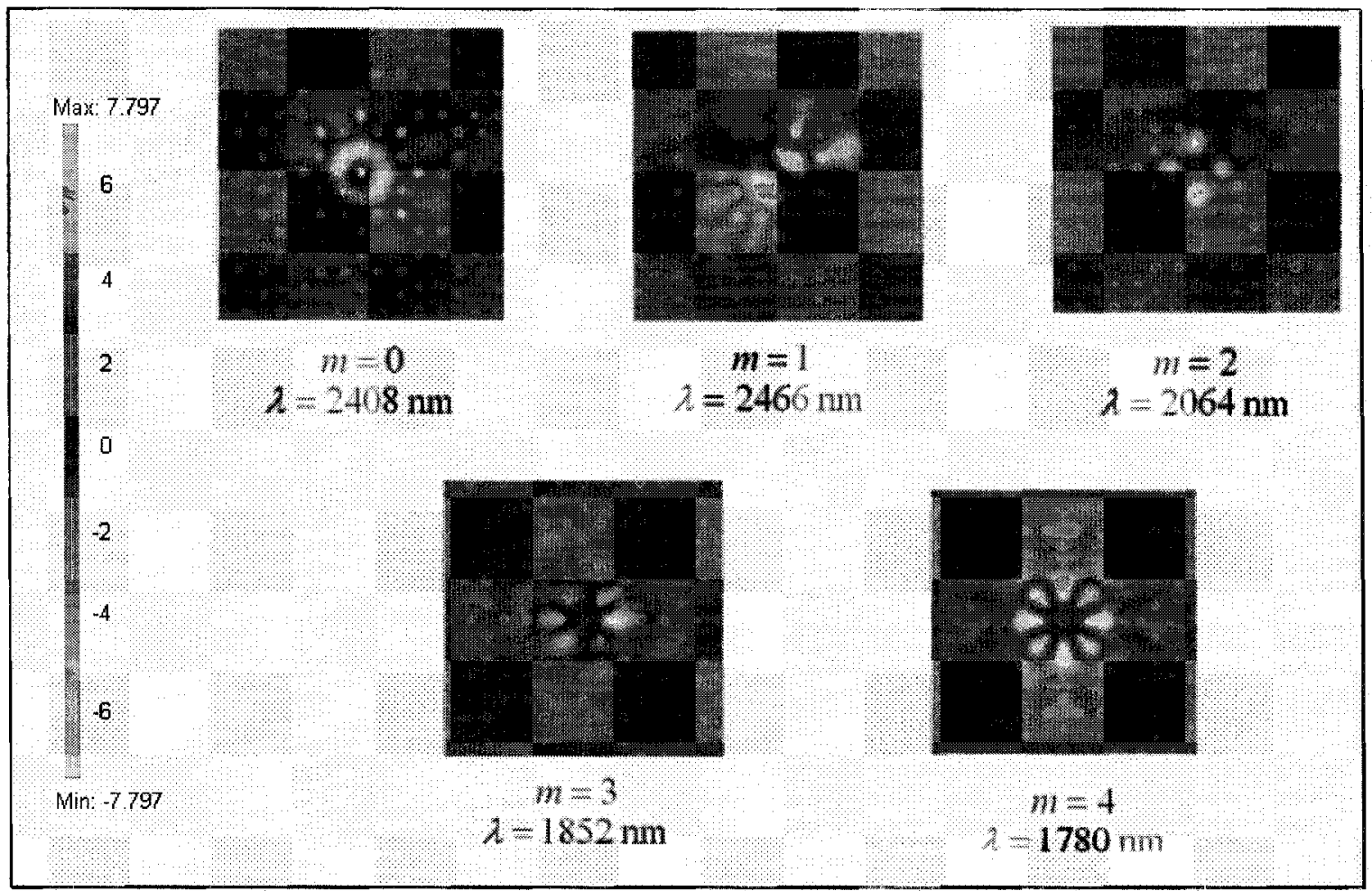

Figure 3.48. Rotational Bloch modes of octagonal (8-fold) PhQ. 


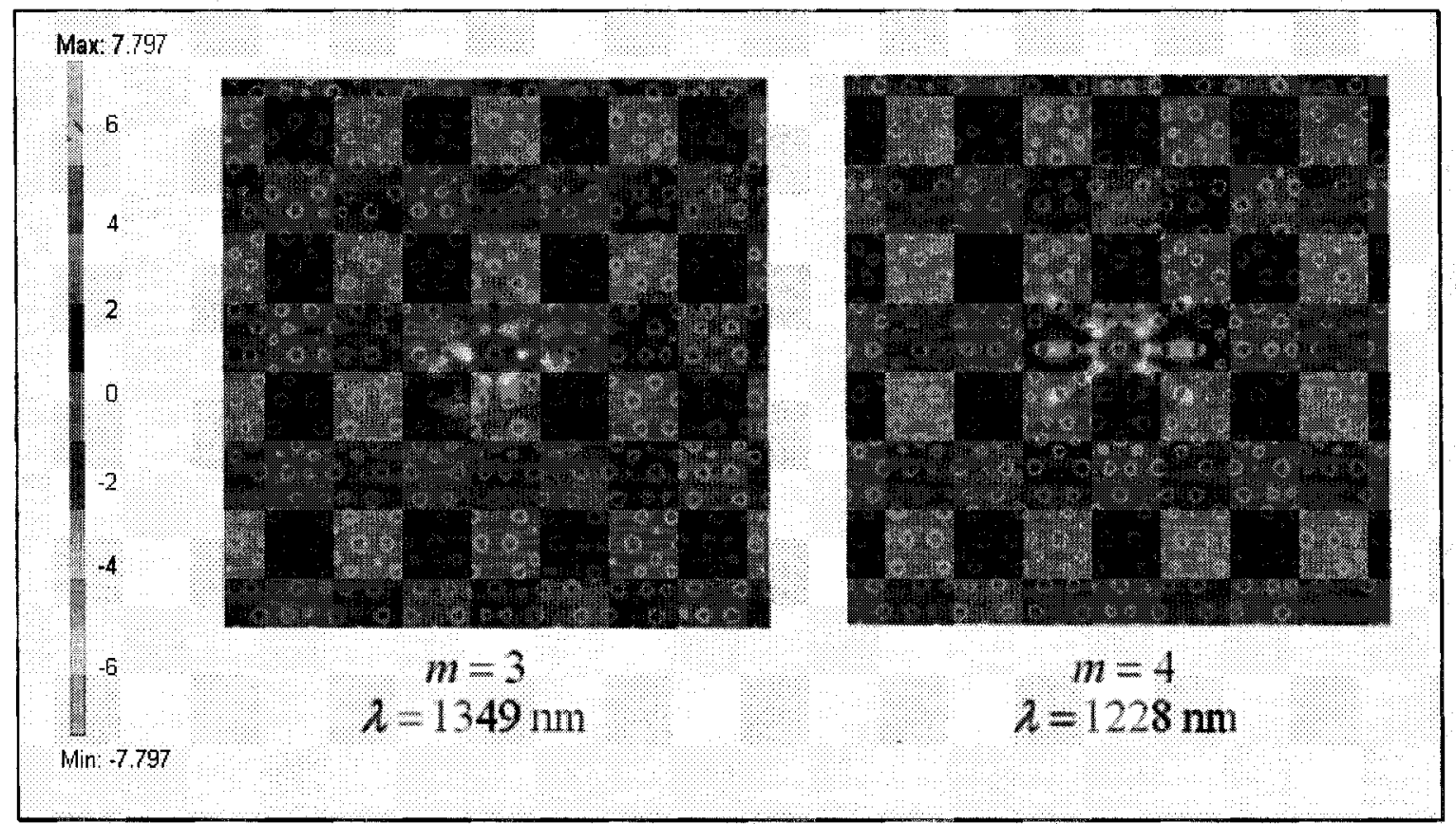

Figure 3.49. Rotational Bloch modes for decagonal (10-fold) PhQs.

These decagonal CAM states were obtained as a predication from the theory proposed in this thesis. Obtaining such states provides more legitimacy to the theory of crystal angular momentum. Another important note is that this optical Lamb shift is seen yet again with the octagonal DFF. The $m=0$ is lower in wavelength than the $m=1$, while the other wavelengths follow the appropriate order.

\subsection{Accessing Symmetries: Coupling Simulations}

\subsubsection{Designing Three Systems of Observation}

This thesis and other work in the literature [25,37] have found that some of the PhQ bandstructure and bandgaps originate from short-range effects. These defect-less localization modes may in fact be the reason behind the bandstructure of $\mathrm{PhQs}$ in general. One can exploit these two effects (short-range effects and localized modes) and build a physical system to measure. Before doing this, however, it would be appropriate to 
simulate the system in question. Three systems will be simulated in preparation for fabrication. The first system will be a $10 \mu \mathrm{m}$ by $10 \mu \mathrm{m} 20 \%$ fill factor PhQ pattern with a silicon photonic wire of a width of $600 \mathrm{~nm}$ crossing the pattern at about $1.7 \mu \mathrm{m}$ from the center. The second system will be a $40 \mu \mathrm{m}$ by $20 \mu \mathrm{m} 20 \%$ fill factor PhQ pattern with again, a silicon photonic wire of a width of $600 \mathrm{~nm}$ crossing the pattern at about $1.7 \mu \mathrm{m}$ from the center. The third system will be a $10 \mu \mathrm{m}$ by $10 \mu \mathrm{m} 20 \%$ fill factor PhQ pattern with a silicon photonic wire of a width of $600 \mathrm{~nm}$ crossing the pattern at the center but having a $3 \mu \mathrm{m}$ gap right at the center of the PhQ. Figure 3.50 shows the three systems to be modeled using FEM.

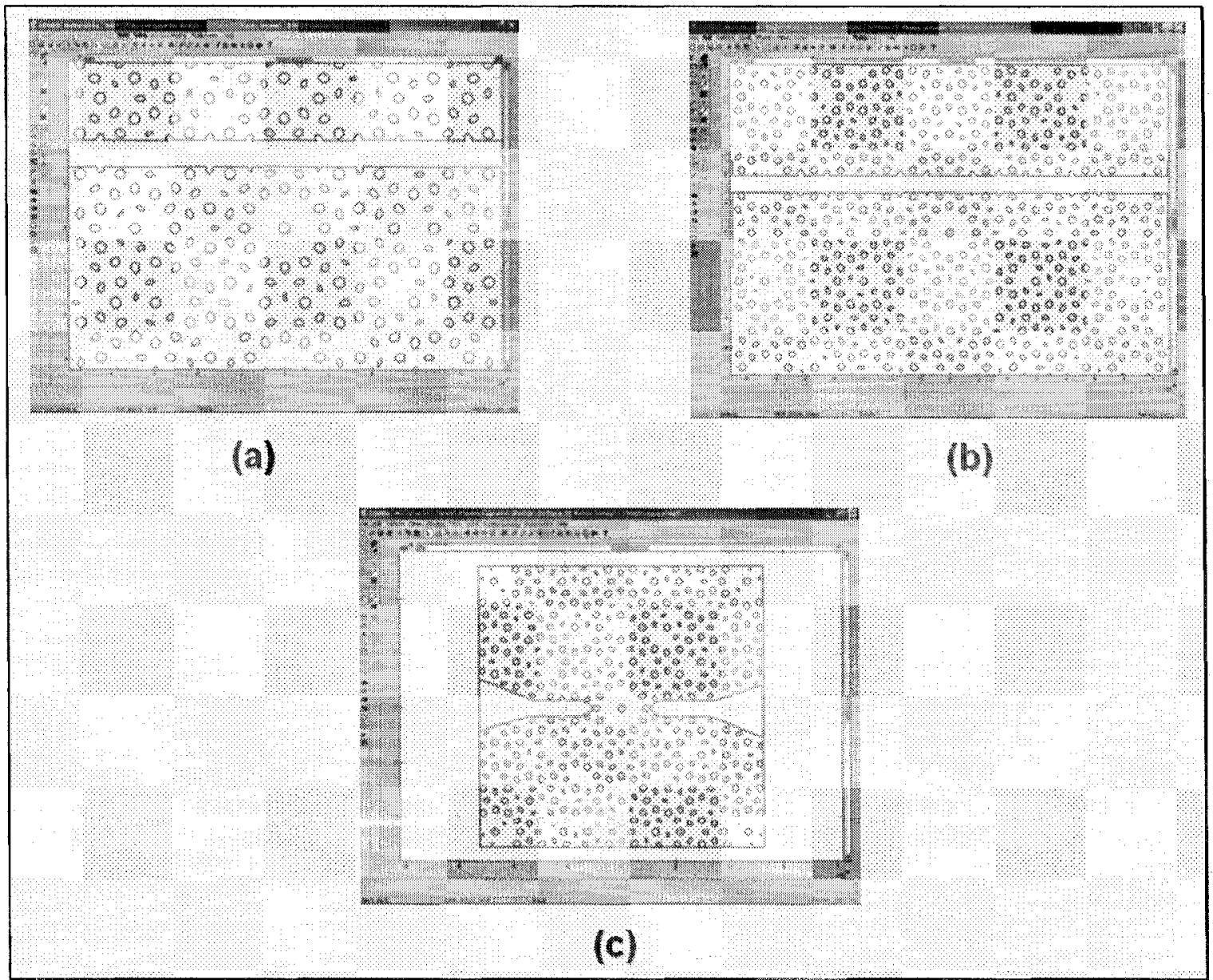

Figure 3.50. Three systems to simulate. (a) $10 \mu \mathrm{m}$ by $10 \mu \mathrm{m} 20 \%$ fill factor PhQ pattern with a silicon photonic wire of a width of $600 \mathrm{~nm}$ crossing the pattern at about $1.7 \mu \mathrm{m}$ from the center. (b) $40 \mu \mathrm{m}$ by $20 \mu \mathrm{m} 20 \%$ fill factor PhQ pattern with a silicon photonic 
wire of a width of $600 \mathrm{~nm}$ crossing the pattern at about $1.7 \mu \mathrm{m}$ from the center. (c) $10 \mu \mathrm{m}$ by $10 \mu \mathrm{m} \mathrm{20 \%} \mathrm{fill} \mathrm{factor} \mathrm{PhQ} \mathrm{pattern} \mathrm{with} \mathrm{a} \mathrm{silicon} \mathrm{photonic} \mathrm{wire} \mathrm{of} \mathrm{a} \mathrm{width} \mathrm{of} 600 \mathrm{~nm}$ crossing the pattern at the center but having a $3 \mu \mathrm{m}$ gap right at the center of the PhQ.

The physical source to be used for measurement is limited in its wavelength range, so what is required is to obtain modes that are accessible for the source. For typical communication channels, $1460 \mathrm{~nm}$ is the lowest wavelength, while $1580 \mathrm{~nm}$ is the highest wavelength. Modes with eigenvalues above this wavelength should be detectable in silicon.

\subsubsection{Simulation Results}

Figure 3.51(b) shows the result of the first system simulation. It seems that the presence of the waveguide has shifted the eigenvalue of the localized modes. This is to be expected, since symmetry is important in the generation of these localized modes and the local symmetry has indeed changed when the waveguide is introduced.

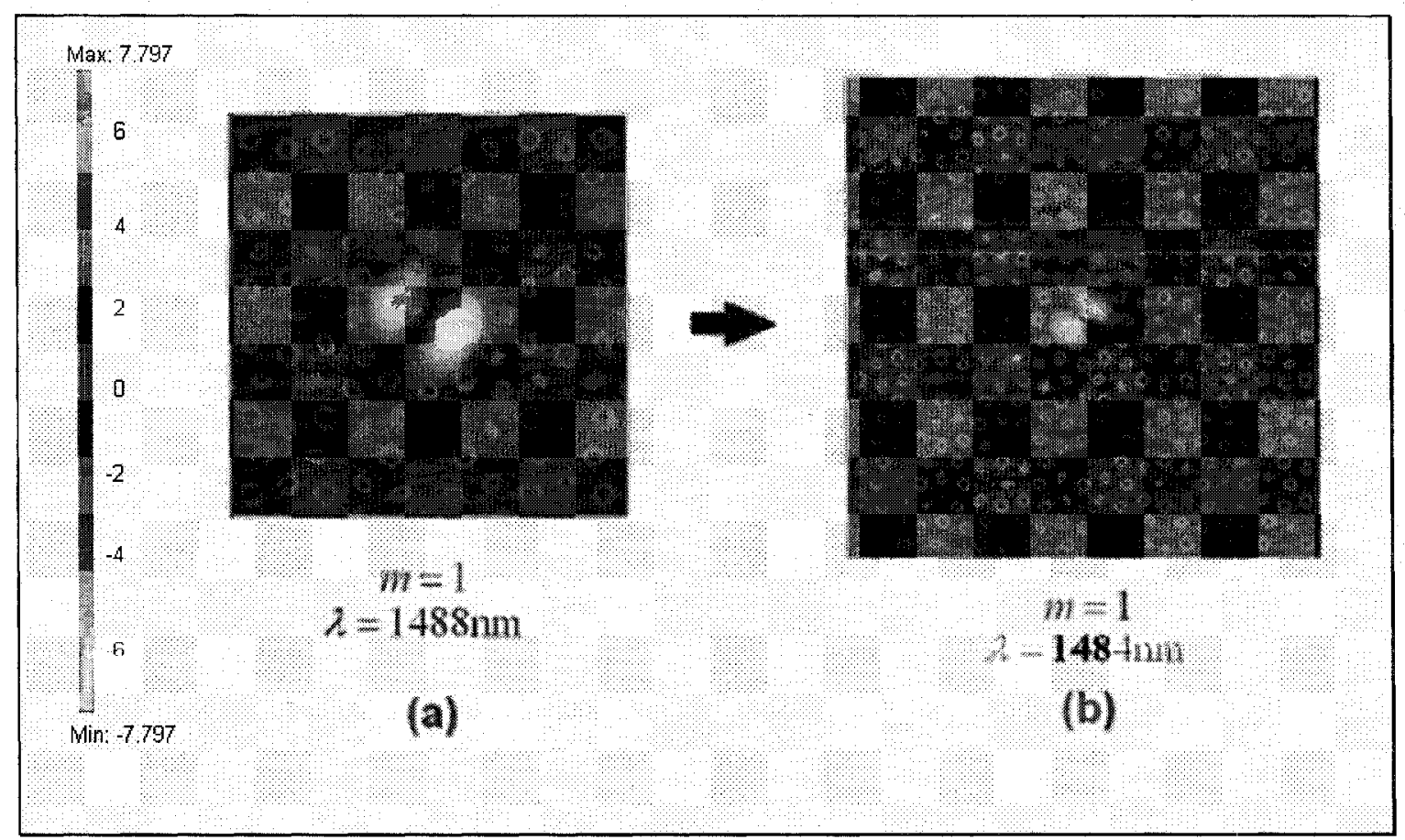

Figure 3.51. Placing photonic wire waveguides near sites shifts eigenvalues. (a) Mode with no waveguide nearby. (b) Mode with waveguide nearby, resulting in wavelength shift. 
The second system is a larger version of the first. This was done to determine if modes that are off-center could be excited. It would be expected that only the $m=0$ or $m=1$ modes would be seen, since the other modes would require lower wavelengths (i.e. higher energies). Figure 3.52 shows the simulation results of the second system. Figure 3.52(a) shows the same mode generated as that in figure $3.51(\mathrm{~b})$, and figure $3.52(\mathrm{~b})$ shows an off-center $m=0$ mode being generated. The third system directs more energy into the creation of these localized modes. Figure 3.53 shows the results of the simulation of the third system. Both $m=1$ and $m=0$ can be excited and note that they are Lamb shifted: the $m=1$ mode has a larger wavelength (i.e. lower energy ) than the $m=0$ mode.

The next step in the thesis is to build such systems and compare them to the simulated results.

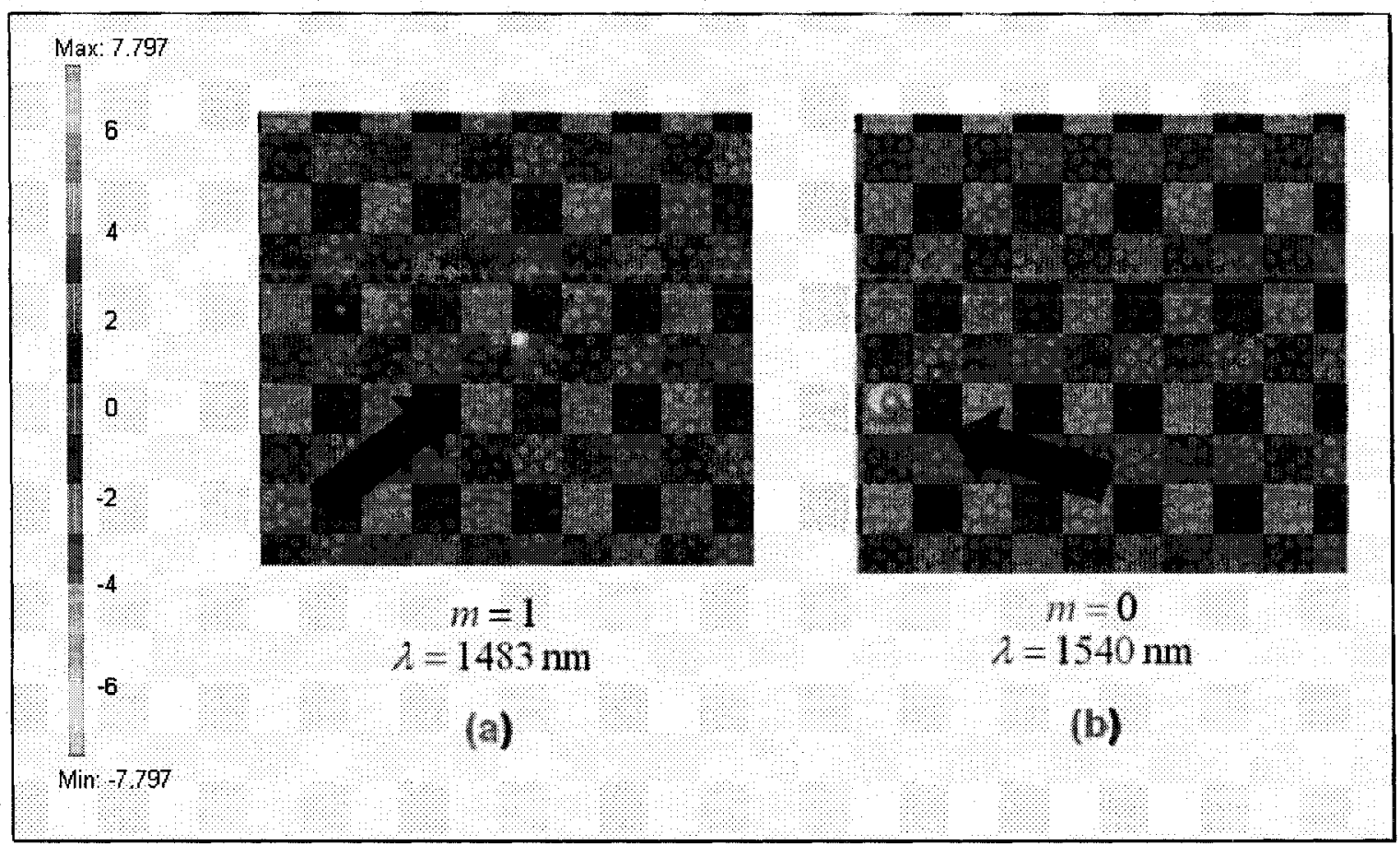

Figure 3.52. Larger PhQ pattern shows two types of localized modes. (a) Arrow points to center localized mode. (b) Arrow points to off-center localized mode. 


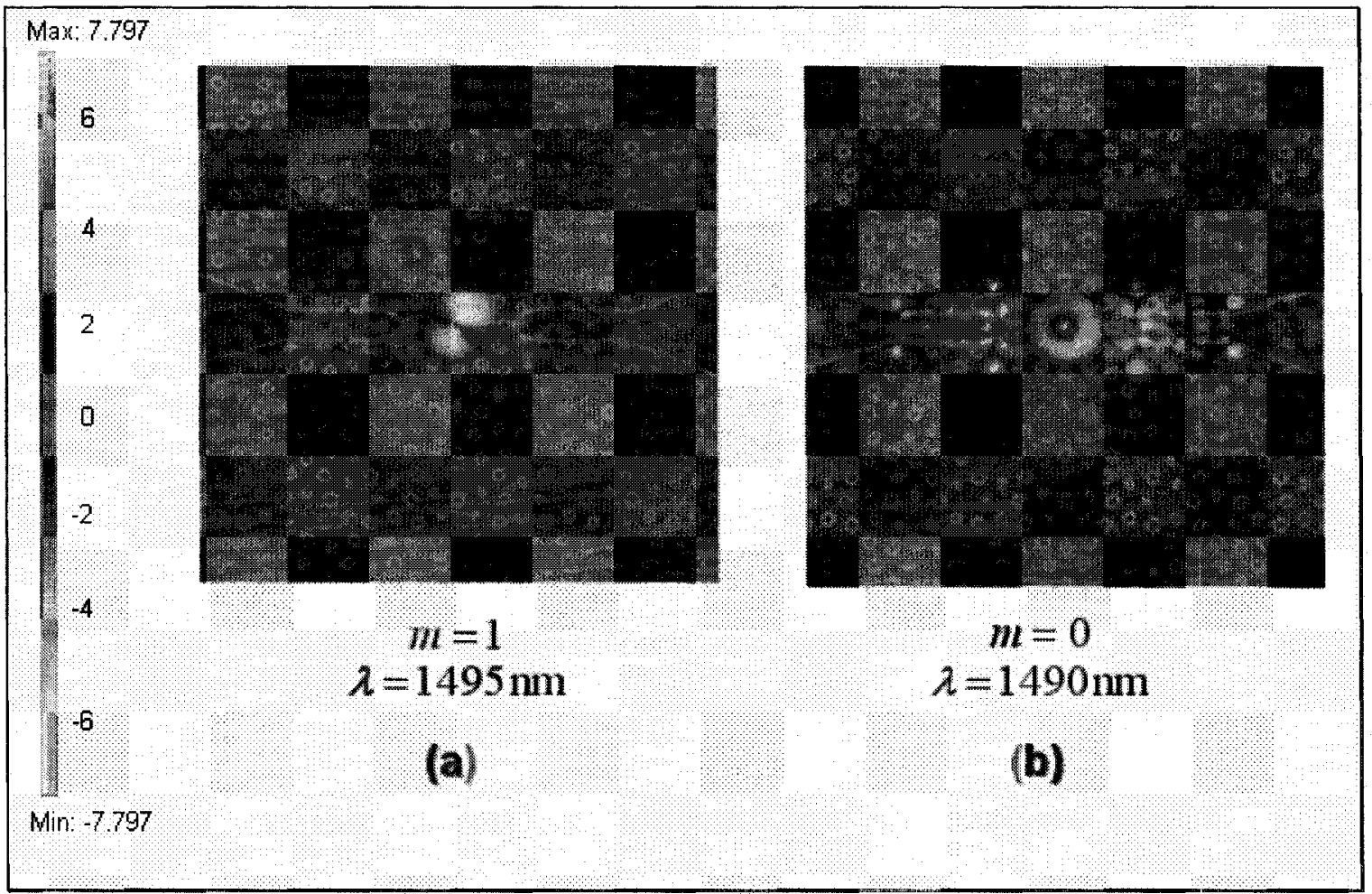

Figure 3.53. Simulation of third system. (a) $m=1$ excited and (b) $m=0$ excited. Note that they are Lamb shifted in terms of wavelength. 


\section{Fabrication: Accessing Symmetries}

This chapter describes the fabrication and measurement of the PhQ structures presented in the previous chapters. Two concepts are important when undertaking a fabrication flow for such structures: (1) building what was simulated with minimal change to the structure, and (2) putting together a fabrication flow with a relatively quick turn around. Previously, these steps were not available for incorporating PhQ structures with integrated optics, which meant that all this had to be developed. Much time was dedicated to establishing a quick and reliable fabrication process and building an experimental testing facility for probing the functionality of PhQ devices. The aim was to demonstrate key attributes of crystal angular momentum in PhQs.

The facility used was Carleton University's Microfabrication Laboratory (MFL). Before integrated photonics was done at Carleton University's MFL, it was used primarily for CMOS VLSI work. It was designed to build micron-level transistors, which by today's standards is quite dated. This meant the equipment (i.e. photolithography, dry-etching, etc.) parameters were all tuned to fabricating electronic devices at the micron scale. Integrated photonics using PhQs demanded a change in these parameters in addition to requiring submicron features. Hence, a great deal of time went into establishing a flow to build PhQs into the MFL. This involved deep knowledge of the equipment at a technical level, such as in understanding how the flow controllers worked on the dry-etching machines, and understanding how the scan coils work on the scanning electron microscope, etc. All this entailed pushing the equipment beyond normal operating parameters. 
The most critical step in realizing the above process is performing electron-beam lithography on a converted scanning electron microscope (SEM).

\subsection{Electron Beam Lithography (EBL)}

\subsubsection{Scanning Electron Microscopy For Writing}

Figure 4.1 shows a picture of Carleton University's SEM system. On the left is the actual

SEM system and on the right is the control and viewing station.

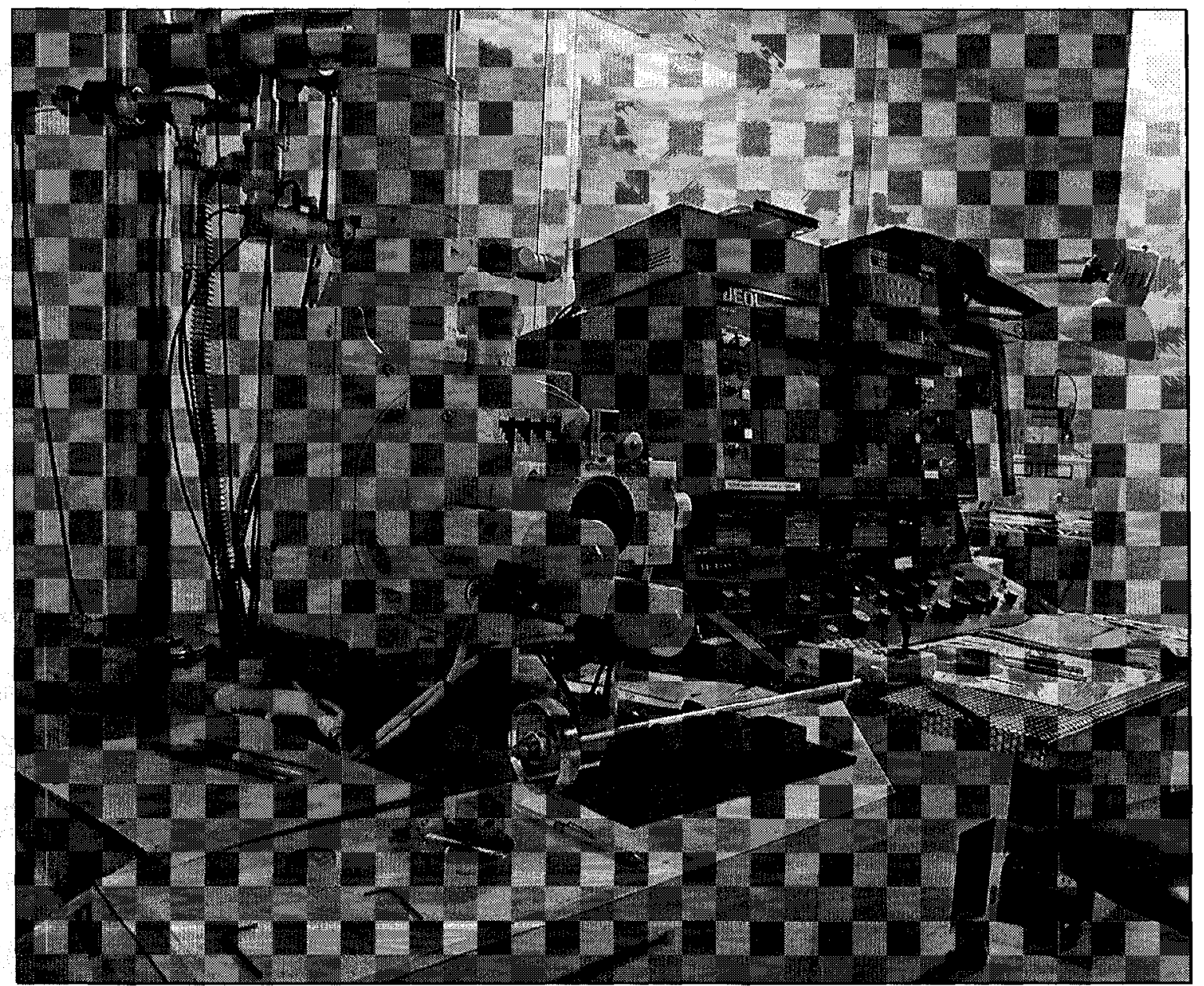

Figure 4.1. Carleton University's Scanning Electron Microscope (SEM) used for electron beam lithography. 
Details on the SEM and its components are presented in Appendix VII. Converting the MFL's SEM into an electron-beam lithography tool was challenging. It required a specialized knowledge of how to shape and prepare the electron beam for writing $\mathrm{PhQs}$ patterns. The innovative work in this thesis demonstrates that some very intricate structures were made with such poor conditions for EBL.

Beam diameter is crucial for SEM writing. As the beam traverses the column, the elaborate system of lens will prevent the beam from diverging. This action results in a demagnification of the original virtual source size,

$$
d_{g}=\frac{d_{v}}{M^{-1}}
$$

where $d_{v}$ is the virtual source diameter, $M^{-1}$ is the demagnification of the column (which is SEM specific), and $d_{g}$ is the resultant beam diameter. The demagnification depends on the beam current from the SEM. Different manufacturers of SEMs have different parameters that yield this demagnification. Typical demagnification values are between 5 and 7 . In this case, with demagnification alone, the smallest beam diameter one can achieve at Carleton University's SEM is about $5 \mu \mathrm{m}$. However, as described later, there are ways to make this smaller in an innovative way.

As the beam makes it way down the column, aberration effects start to cause divergence of the beam. There are two aberrations in particular: spherical and chromatic. The spherical aberration is a result of the nonuniformity of the magnetic lens. The outer zones of the lens tend to focus more strongly than the center, so this adds to the divergence. The relation for finding the diameter due to spherical aberration, $d_{s}$, is 


$$
d_{s}=\frac{1}{2} \alpha^{3} C_{s}
$$

where $C_{s}$ is the spherical aberration coefficient of the final lens, and $\alpha$ is the convergence half-angle of the beam at the target. Chromatic aberration occurs when the beam energy spread is larger so that lower energy electrons are focused more strongly then higher energy ones. The relation for finding the diameter due to chromatic aberration, $d_{c}$, is

$$
d_{c}=\alpha C_{c} \frac{\Delta V}{V_{D}}
$$

where $C_{c}$ is the chromatic aberration coefficient of the final lens, $\Delta V$ is the energy spread of the electrons, and $V_{b}$ is the acceleration voltage. These two aberration effects are respectively dependent upon the design of the lens and sources. The last effect that adds to the divergence comes from the quantum mechanical nature of the electron. Its wavelength is going to be related to the acceleration voltage of the SEM,

$$
\lambda_{e}=\frac{1.2}{\sqrt{V_{b}}}
$$

where $\lambda_{e}$ is the electron wavelength (in $\mathrm{nm}$ ) and $V_{b}$ is the acceleration voltage. Even though it is much smaller than the wavelength of light, this wavelength can still limit the beam diameter by classical diffraction effects in very high-resolution systems. The relation for finding the diameter due to diffraction, $d_{c}$, is,

$$
d_{d}=\frac{0.6 \lambda_{e}}{\alpha} .
$$


Taking all these into consideration, the final beam diameter, $d$, is

$$
d=\sqrt{\left(d_{g}^{2}+d_{s}^{2}+d_{c}^{2}+d_{d}^{2}\right)} .
$$

The physical values for Carleton University's SEM is $C_{s}=60 \mathrm{~mm}$ and $C_{c}=40 \mathrm{~mm}$, a demagnification of 1388.89 times, and an acceleration voltage of $40 \mathrm{kV}$, gives,

$$
\begin{gathered}
d_{g}=18 \mathrm{~nm} \\
d_{s}=3.75 \mathrm{~nm} \\
d_{c}=15 \mathrm{~nm} \\
d_{d}=0.96 \mathrm{~nm}
\end{gathered}
$$

which gives an overall diameter of $d=25 \mathrm{~nm}$ with a beam current of $20 \mathrm{pA}$. This diameter is bigger than the resolution limit of the electron-beam resist, which is about 10 $\mathrm{nm}$. It would be ideal to have a beam diameter close to this value to improve its resolution.

There was a relationship discovered deep within the SEM manual that related electron source brightness to beam current and the diameter of the beam was part of this equation,

$$
\beta=\frac{0.4 i}{\alpha^{2} d^{2}}
$$

where $\beta$ is the source brightness, $i$ is the beam current, $\alpha$ is the half-angle described previously, and $d$ is the beam diameter. In this particular case, the source brightness is $10^{5} \mathrm{~A} / \mathrm{cm}^{2} / \mathrm{sr}$. The half-angle is $\alpha=0.005$ radians. It is calculated from the knowledge 
of the aperture diameter $(\sim 50 \mu \mathrm{m})$ and the working distance $(\sim 5 \mathrm{~mm})$. From equation 4.11 , in order to get a beam diameter of $10 \mathrm{~nm}$, one needs a beam current of $3 \mathrm{pA}$. Finding 4.11 gave the author the capability to write intricate and small feature sizes for PhQs studied in previous chapters.

\subsubsection{Electron beam resist}

This beam current is quite low, which means that the selection of the electron-beam resist is important. The electron beam resist that was originally used in the beginning stages of EBL was polymethyl methacrylate (PMMA). It has a high dose requirement for exposure, $350 \mu \mathrm{C} / \mathrm{cm}^{2}$, and if used at a beam current this low would take too much energy to pattern. Fortunately, another resist was available to Carleton University provided by the National Research Council (NRC) of Canada. This resist is known as ZEP. ZEP consists of a copolymer of -chloromethacrylate and -methylstryrene. It's exposure dose is an order of magnitude lower than PMMA, $30 \mu \mathrm{C} / \mathrm{cm}^{2}$, which means that it is useful and efficient at a beam current of $3 \mathrm{pA}$. Like PMMA, it is a positive resist, which means that when exposed, the resist dissolves in a developer. The developer for ZEP used at Carleton University is o-Xylene. The undiluted ZEP thickness is about 300 $\mathrm{nm}$ which is obtained from a spin-on rate of $6000 \mathrm{rpm}$ for 30 seconds. Further details about applying the resist to the sample and preparing the covered sample for the SEM writing can be found in Appendix VIII. 


\subsection{Image Processing for Electron-Beam Writing}

\subsubsection{Pattern Preparation}

The software that generates the PhQ images must be able to output the image in one of the standard file formats: JPEG or GIF. For example, the Mathematica® code used to generate the PhQ image in figure 4.2 can output the image as a JPEG file.

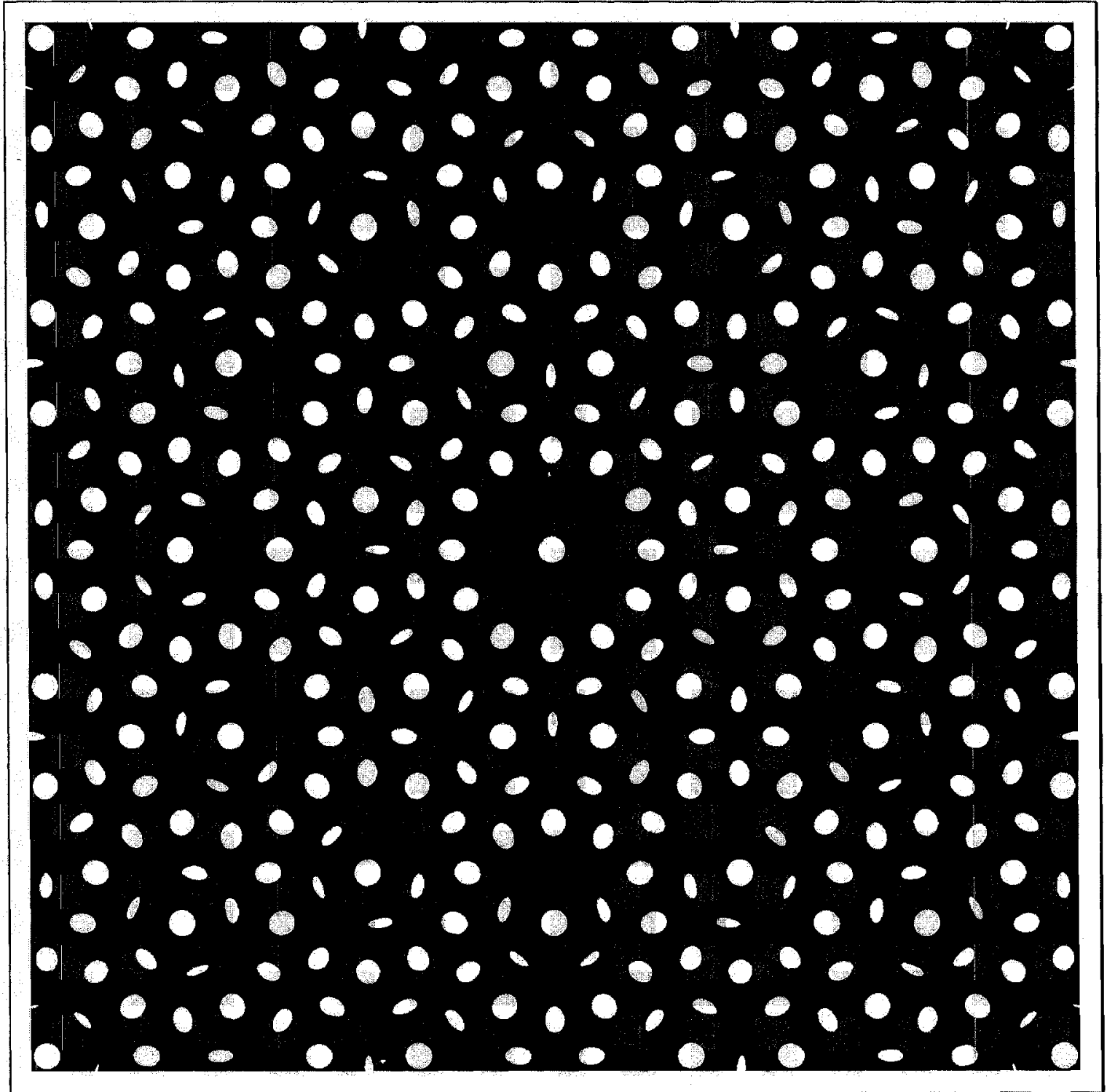

Figure 4.2. PhQ generated from Mathematica. This is the $16 / 24$ or $20 \%$ fill factor. 
It is important to have the file in black and white so that one knows what color represents exposed regions and what color represents unexposed regions. If one is making posts with a positive resist, one has to write the area around the post. Hence, one has to write the inverse image for posts (direct image for hole structures). The PhQ structure studied here will be made from posts in order to explore the large bandgap for the TM polarization. The generated image is then loaded into an image processing tool, as shown in figure 4.3 (Adobe® Photoshop® Elements $\left.{ }^{\circledR} 2.0\right)$.

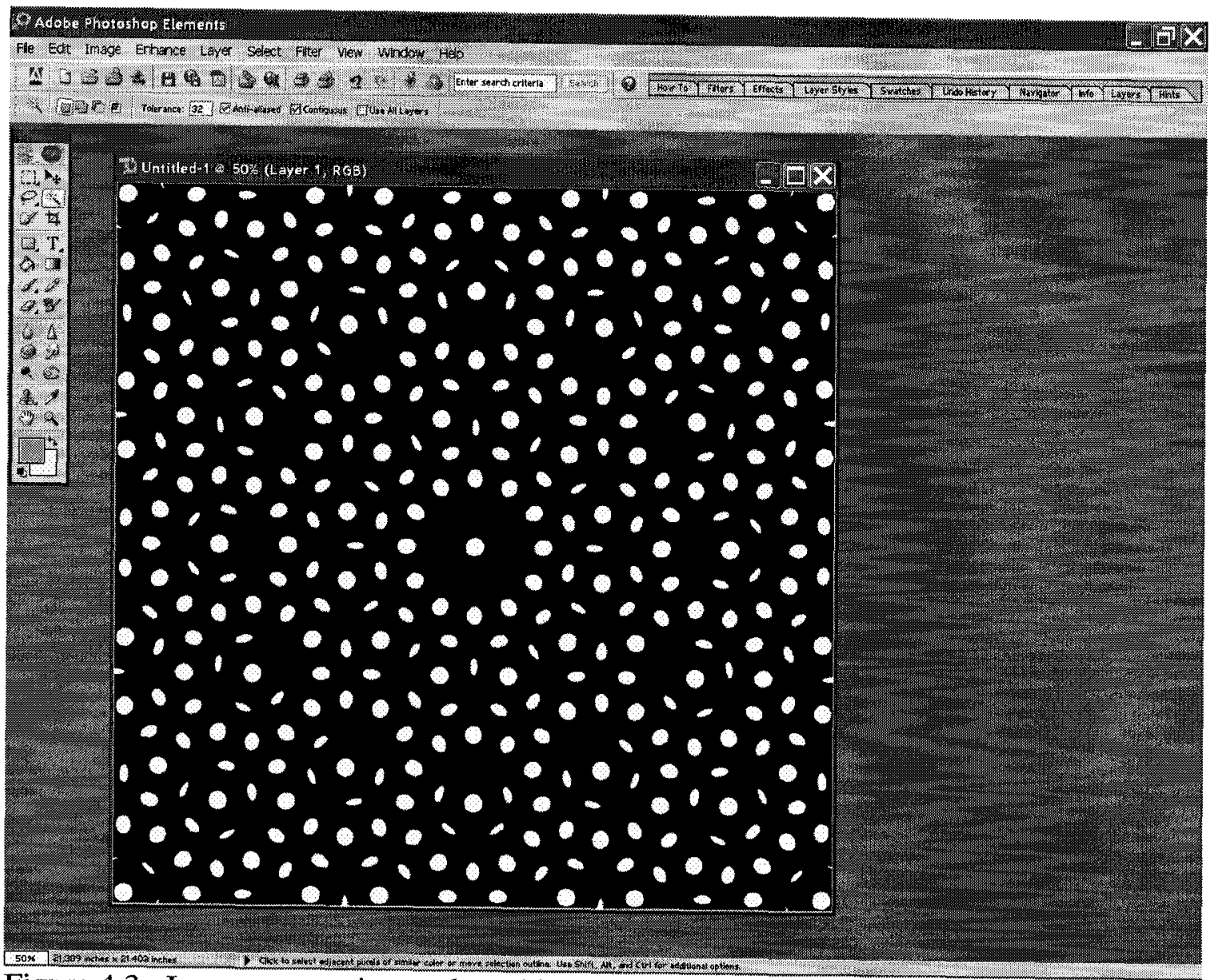

Figure 4.3. Image processing tool working on a PhQ structure ready for writing.

This allows the user to use image filters for sharpening contrasts, flattening, and other image processing techniques that may help in preparing the image for electronbeam writing. The last step in this process is resizing the image in accordance with a 
'pixel to nanometer' ratio. The ratio selected in this work is $1: 10$, that is, one pixel is 10 nanometers. If the pattern is to be $10 \mu \mathrm{m}$ by $10 \mu \mathrm{m}$, then the conversion ratio dictates that the image be resized to 1000 pixels by 1000 pixels. The file is then saved and opened in LinkCad®, an image-conversion software tool, where it is converted into a DXF file format. The new file is then converted into CIF format using CleWinß, as shown in figure 4.4. Once the CIF file has been generated, the pattern can then be electron-beam written using the NPGS software.

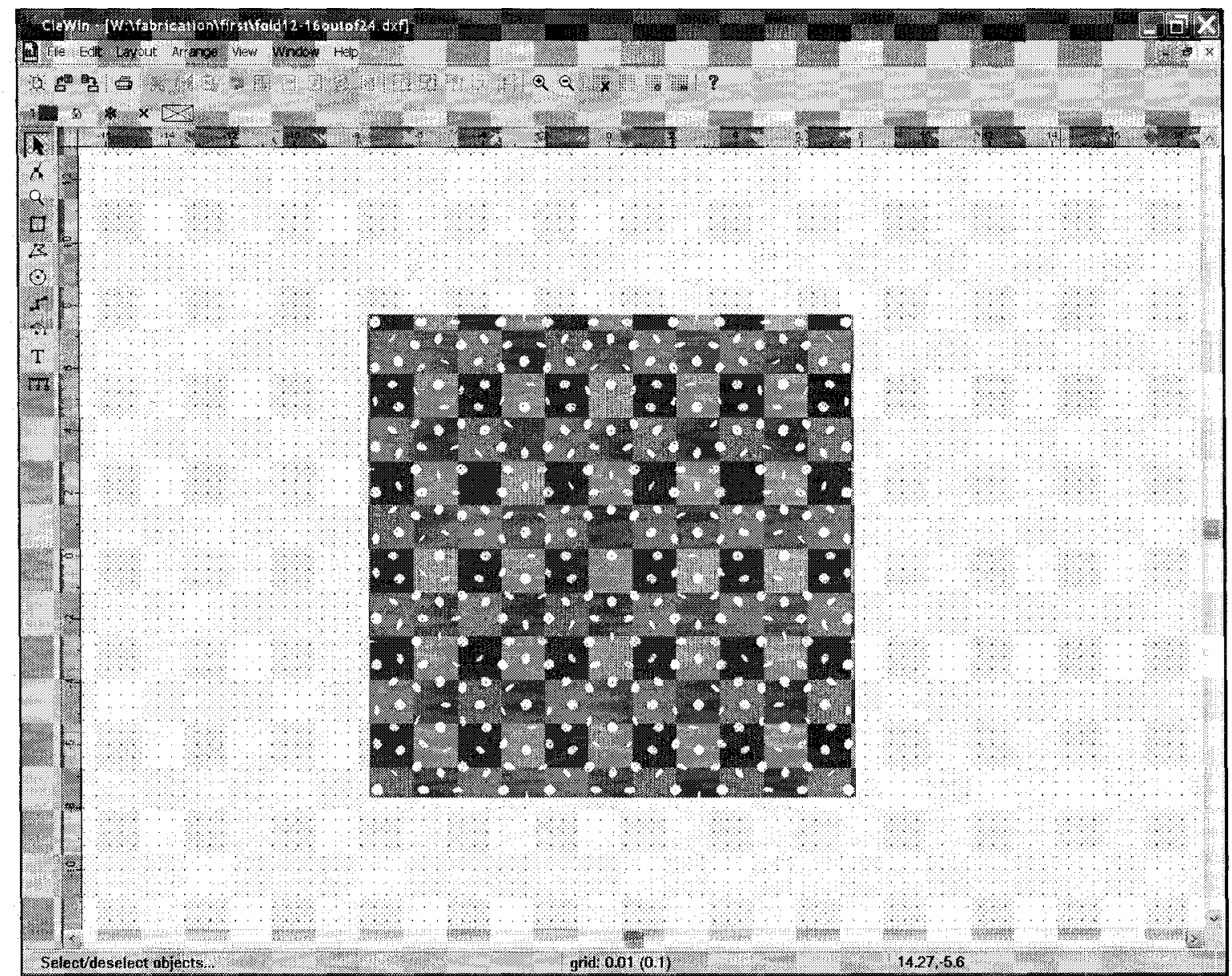

Figure 4.4. CleWin editor showing converted PhQ image. 


\subsubsection{Nanometer Pattern Generation Software (NPGS)}

The NPGS software [52] is a user-friendly environment which facilitates the writing of complex structures from nanometer feature sizes up to the maximum field of view of the microscope. There are three basic steps to the pattern generation process: pattern design, parameter run file creation, and pattern writing. The pattern design has been described in the previous sections and resulted in a CIF file. This CIF file needs to be converted into a format used by the NPGS system, and must produce the file format required by the SEM run file. Figure 4.5 shows an example of a specific run file. In this case, there is only one pattern being written since Page 1 of 1 is written on the top right hand corner. The first line is the layer information line. Layers in the MRF file allow the user to specify different writing parameters for different areas in the pattern. The $w, p, c, s$ letters for the layer specification specifies whether the beam blank will be electronically activated between every exposure point $(w)$, whether a pause will be done before writing $(p)$, whether the beam blank will not be electronically activated between every exposure point (c), or whether the layer (s) should be bypassed altogether. The next line, Origin Offset, allows one to offset the individual layer within a pattern relative to the center of the pattern. The Magnification entry is calculated when the transcoding between CIF and DC2 is done. 


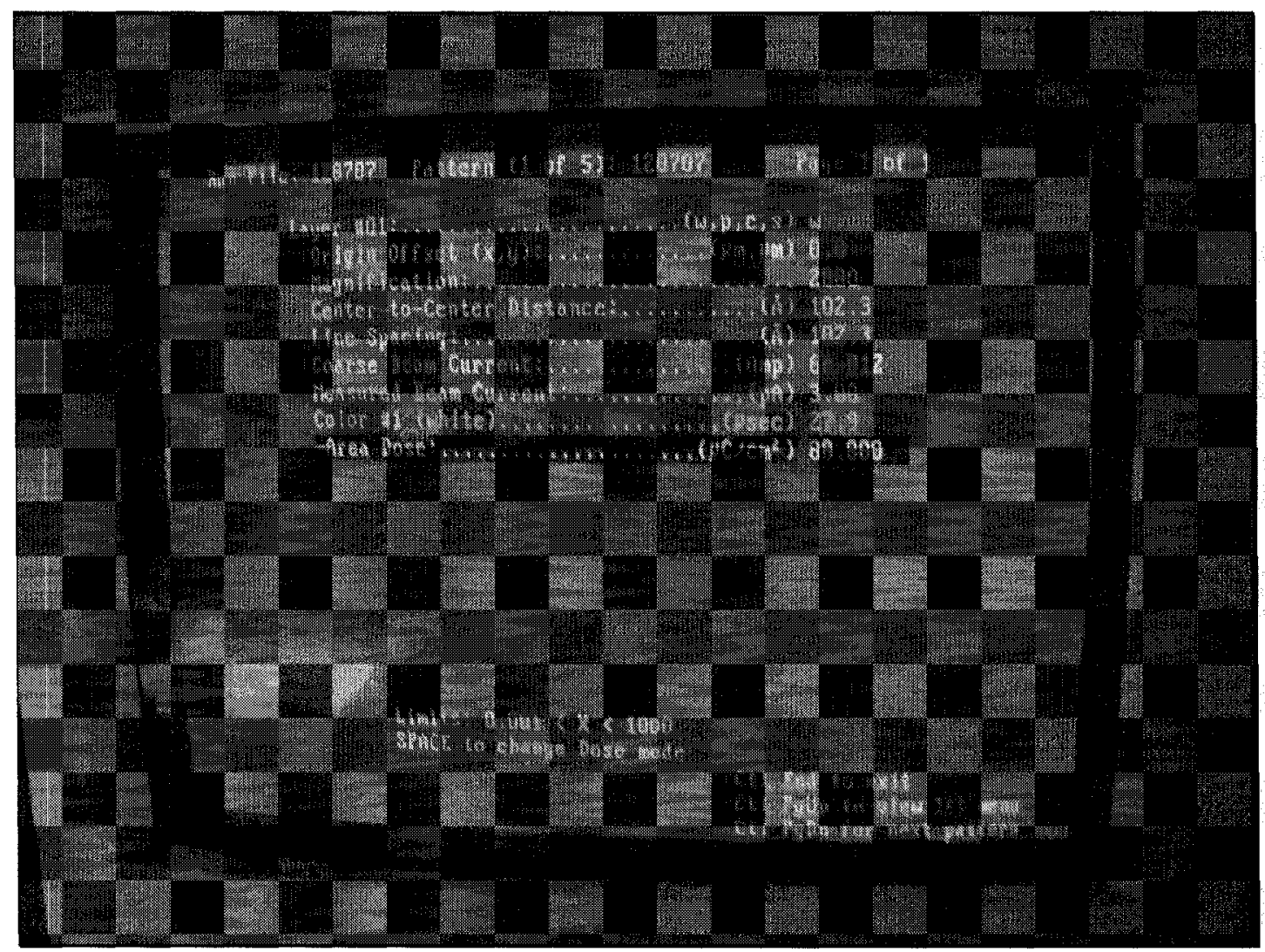

Figure 4.5. Run file exposure parameter page.

The magnification number is calculated from the largest feature length in the CIF file. For example, the magnification shown in figure 4.5 is calculated for a pattern that is $45 \mu \mathrm{m}$ by $26 \mu \mathrm{m}$. The largest size in the pattern is $45 \mu \mathrm{m}$. The JSM- 840 has a $90 \mathrm{~mm}$ field of view at $1 x$ magnification. Hence,

$$
\operatorname{mag}=\frac{9 \times 10^{-2}[\mathrm{~m}]}{[\text { field of view }]}=\frac{9 \times 10^{-2}[\mathrm{~m}]}{4.5 \times 10^{-5}[\mathrm{~m}]}=2000
$$

where $2000 \mathrm{x}$ is the magnification required for an area of $45 \mu \mathrm{m}$ by $45 \mu \mathrm{m}$ that contains the pattern. The size of a pixel in this field of view is,

$$
\underset{\text { size }}{\text { pixel }}=\frac{[\text { field of view }]}{[\text { bit size of DAC }]}=\frac{4.5 \times 10^{-5}[\mathrm{~m}]}{2^{16}}=6.87 \times 10^{-10}[\mathrm{~m}]=6.87 \AA .
$$


This is the smallest addressable area of the field of view. This poses two major problems. The first problem is that the beam diameter is much larger than this - almost a couple of orders of magnitude larger. If each pixel is addressed, the cumulative exposure rates will wash the pattern away. The second problem is that the timing required to address each pixel will far exceed the scan coil settling times (i.e. the deflection bandwidth) of the JSM-840 SEM. The fastest allowable scan coil settling time is 10 $\mu \mathrm{sec}$.

The NPGS program can solve these problems by only selecting some pixels for exposure. This process can virtually enlarge the pixel area. If one selects a virtual pixel size of $10 \mathrm{~nm}$, then this means that there will be 15 addressable pixel sizes left unexposed in between exposed pixels.

The next two entries after magnification in figure 4.5 are the center-to-center distance and line spacing. This is the respective horizontal and vertical distance between exposure points. When one changes these entries, the other parameters will change as well.

The next entry is the measured beam current and is very important because it determines the effective beam diameter in equation 4.11. For this particular work, it was found to be $3 \mathrm{pA}$. The exposure time is calculated once the exposure dose is determined.

The resist used here is the high contrast ZEP520A from Nippon Zeon Co. As stated earlier, its sensitivity is much lower than PMMA. Sensitivity is defined as the point in which all the film is removed. It depends upon the film thickness and acceleration voltage of the SEM. The sensitivity of ZEP is about $30 \mu \mathrm{C} / \mathrm{cm}^{2}$ at $25 \mathrm{kV}$. This is not the best acceleration voltage to use because as the electron beam enters the 
ZEP it will scatter, and if the acceleration voltage is not high enough, the beam will widen while still traversing the resist. This leads to negatively slanted sidewalls and affects the dry etching profile of the material under the resist. Resist and substrate scattering is important as it affects the final pattern produced. As shown in figure 4.6, the lower acceleration voltage $(10 \mathrm{kV})$ has poor penetration depth and the beam is considerably widened. Higher accelerating voltage $(>20 \mathrm{kV})$ will give less widening in the resist but may lead to secondary exposure of other parts of the pattern (proximity effect).

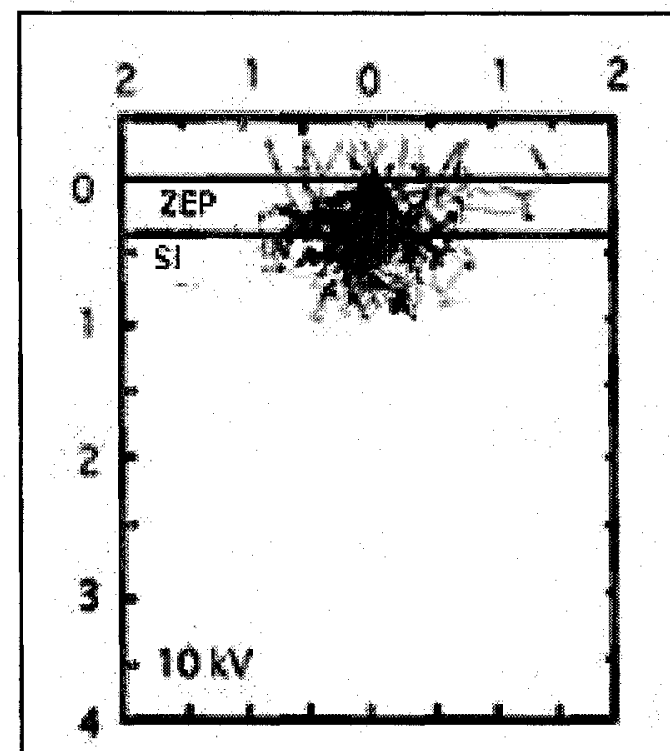

(a)

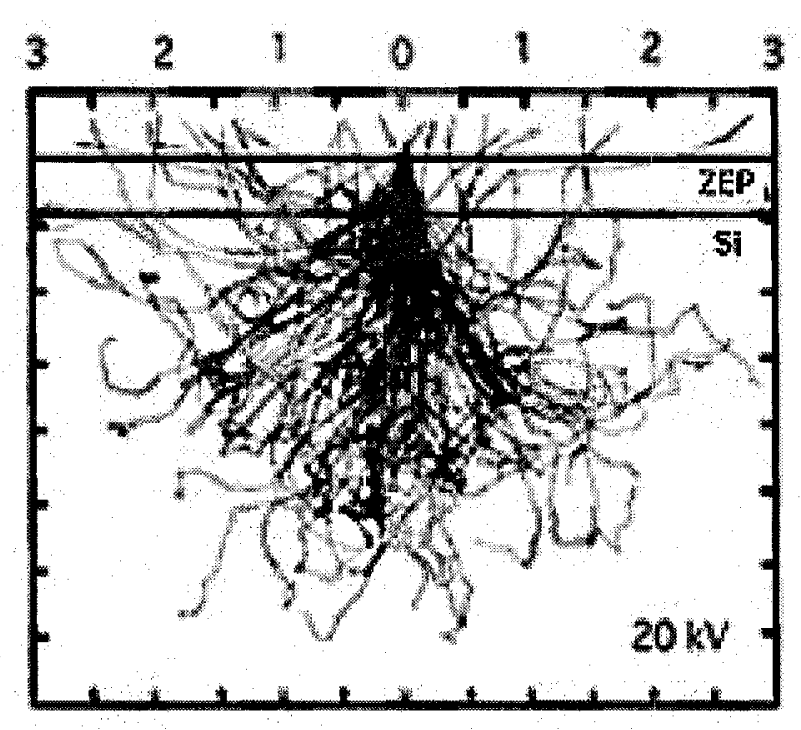

(b)

Figure 4.6. Simulation of electron scattering in resist on a silicon substrate at (a) $10 \mathrm{kV}$ and (b) $20 \mathrm{kV}$ [65].

A useful relation between the diameter widening effect and the acceleration voltage is,

$$
d_{f}=0.9\left(\frac{R_{t}}{V_{b}}\right)^{\frac{3}{2}}
$$


where $d_{f}$ is the amount by which the diameter widens in nanometers, $R_{t}$ is the resist thickness in nanometers, and $V_{b}$ is the acceleration voltage in kilovolts. In this case, the resist thickness was $300 \mathrm{~nm}$ and $V_{b}$ was $39 \mathrm{kV}$, which is near the maximum at which this SEM can run. The amount by which the diameter widens is $19 \mathrm{~nm}$. This means that if the beam diameter is $10 \mathrm{~nm}$, it will be almost $30 \mathrm{~nm}$ as it exits the resist. It is still a large negative slope, but dry-etching compensates because generally speaking, dry-etching gives a positive slope to resists. ZEP is also 2.5 times more resistant to dry etching than PMMA. PMMA's etch rate is on par with silicon's etch rate in Carleton University's dry etch system, which is about $300 \mathrm{~nm} / \mathrm{min}$. This means one can etch deeper with ZEP than with PMMA.

It has been found from this work, that at the acceleration voltage used, a good dose for the ZEP is between $80 \mu \mathrm{C} / \mathrm{cm}^{2}$ and $120 \mu \mathrm{C} / \mathrm{cm}^{2}$. This means that about 500 to 750 electrons are required to fully expose a $10 \mathrm{~nm}$ by $10 \mathrm{~nm}$ area. A lower electron count will result in the pattern being sensitive to the variation of electron numbers and will then become uneven. With a dose of $80 \mu \mathrm{C} / \mathrm{cm}^{2}$, one can then find the exposure time, from the following relation,

$$
t=\frac{(\text { dose })(\text { pixel area })}{(\text { beam current })}=\frac{(80)\left(10^{2}\right)}{(3)}=27.9 \mu \mathrm{sec} .
$$

\subsubsection{Fine Tuning Parameters}

Before any of this work on PhQ structures was done at Carleton University, the SEM was used for writing simple structures such as Bragg gratings, as shown in figure 4.7. However, the desired pitch of the grating and grating length were not achievable at 
that time. Later work on 2D PhCs was attempted but with very course features, as shown in figure 4.8. During this work on 2D PhCs, a study was done on determining if stitching was possible using the Carleton University SEM. This is important when trying to write optical waveguides that are long enough to reach the sample edges for optical fiber coupling.

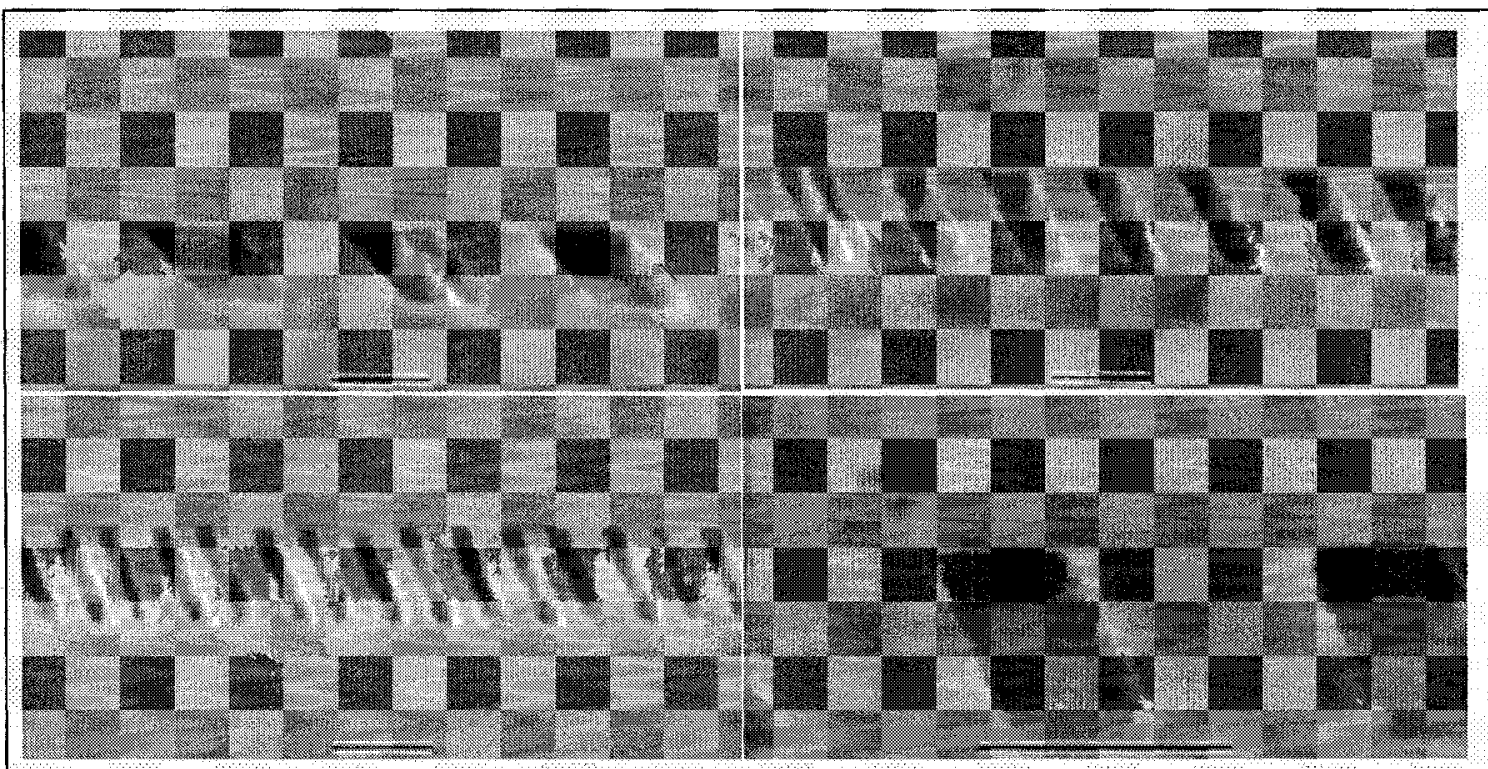

Figure 4.7. Optical gratings done in the first few days of conversion of Carleton University's SEM. Bar length is one micron. 


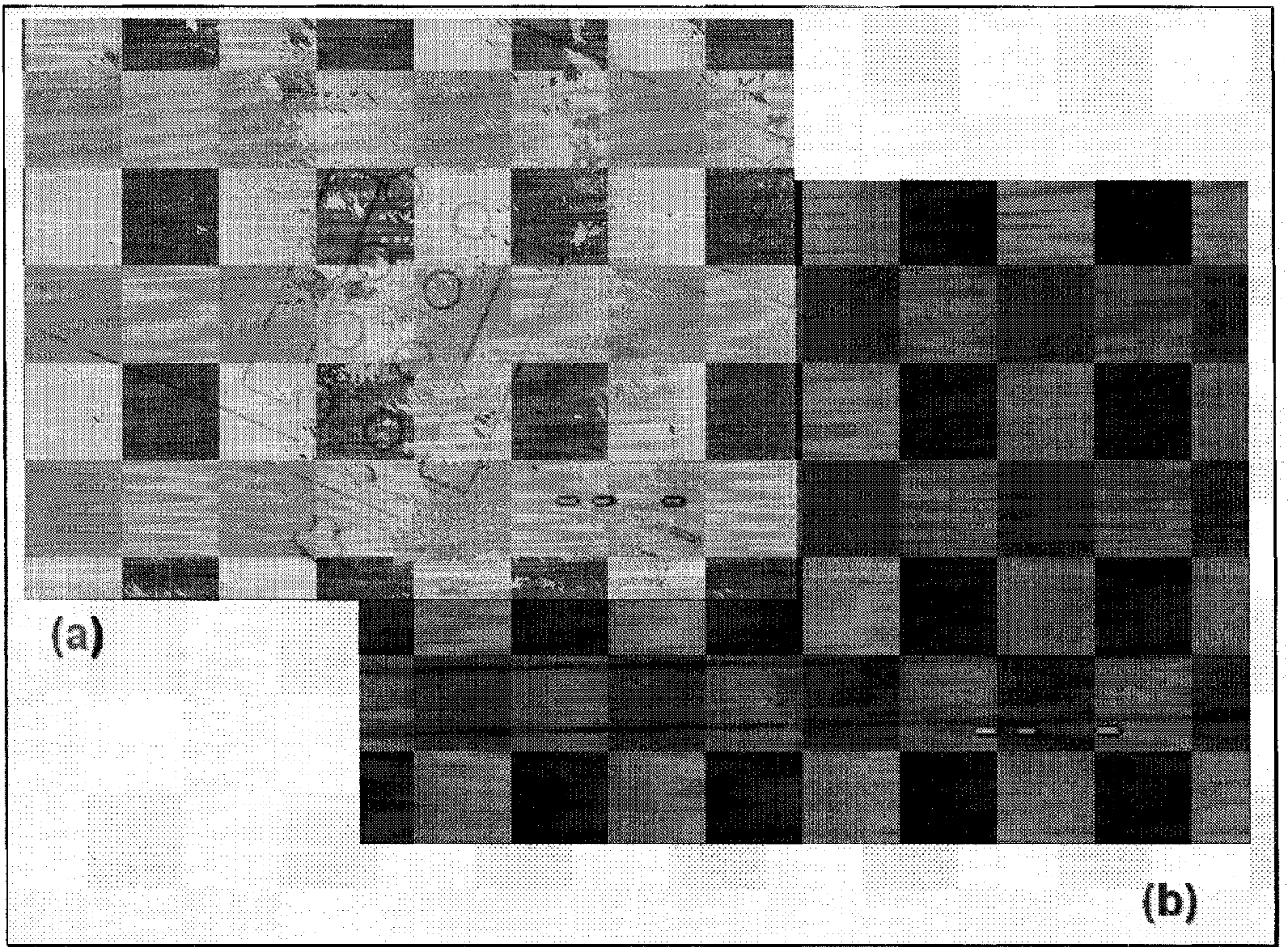

Figure 4.8. First images of writing 2D PhCs. (a) One of the first exercises and (b) writing a "waveguide" along with the $\mathrm{PhC}$. Bar lengths are ten microns.

As it turns out, the course movement of the sample platform within the SEM restricted the stitching process, as shown in figure 4.9. Two options remain: (1) gain access to an electron beam writer or; (2) combine EBL with available photolithography techniques. The first option is costly, time-consuming, and impractical for prototype work. The second option was explored and successfully implemented. The microstructures will be done by EBL and the waveguide design will be added later through photolithography. 


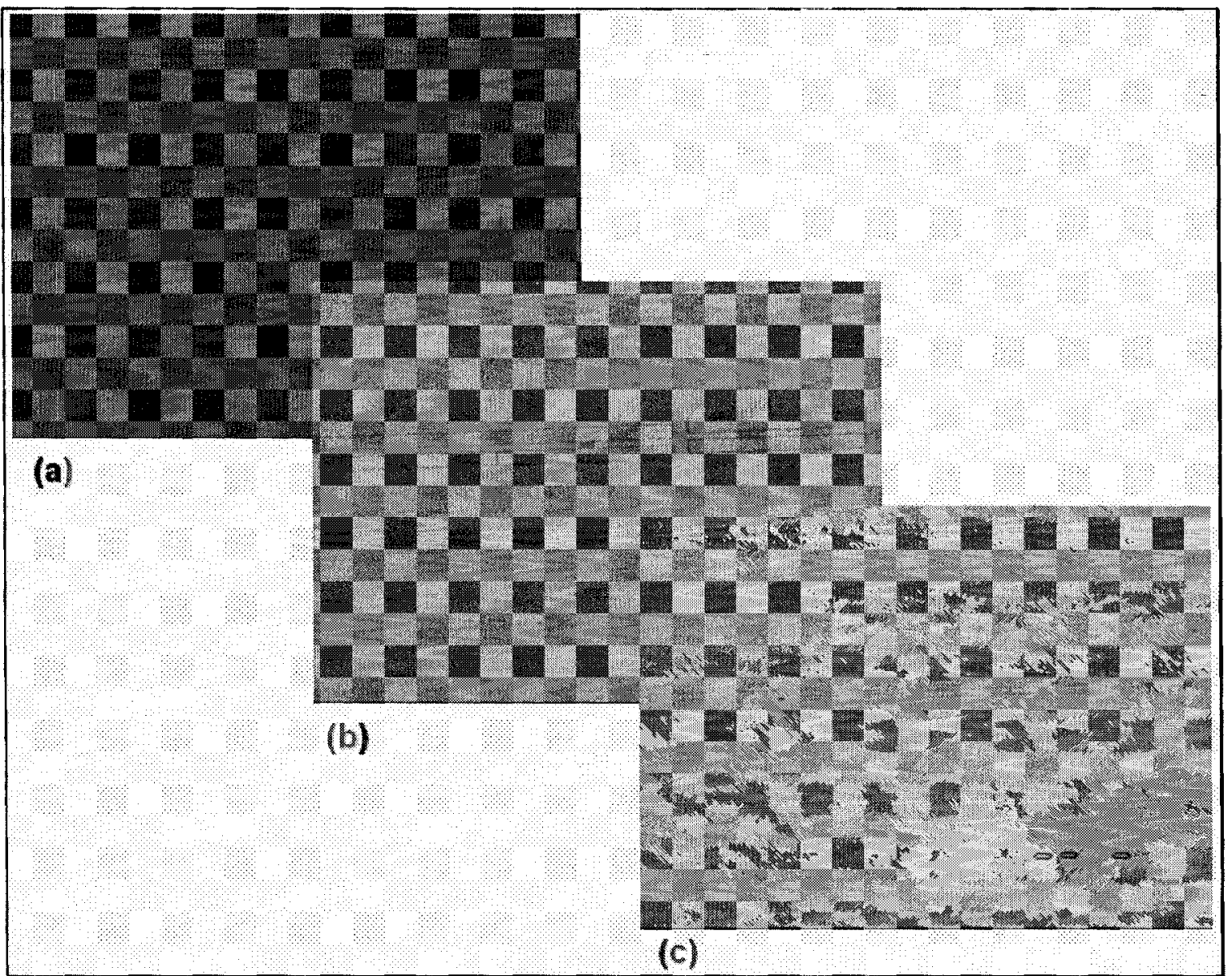

Figure 4.9. Stitching attempts by converted SEM. Bar lengths are ten micron.

Figure 4.10 shows an evolution of writing 2D PhC structures. Image (a) of figure 4.10 is a close up of the structure in figure 4.8 . The holes are very large, $500 \mathrm{~nm}$, and not appropriate for the $1550 \mathrm{~nm}$ optical range. As the electron-beam write parameters are changed, smaller holes can be obtained, but other issues start to arise, such as the proximity effect. Image (b), (c) and (d) all show unintended write defects. They all had too much exposure dose. Once the right exposure was found, namely between 80 and $120 \mu \mathrm{C} / \mathrm{cm}^{2}$, better structures could then be developed. Image (e), and its close up (f), show better structures. Image (g) and (h) show isometric pictures of 2D PhCs in resist. Image (i) is a close up of the hole structures. Image (j) shows other defect type structures as well. Image (k), and its close up image (l), show the sidewalls of the resist. It shows 
that the development proceeded to the substrate surface, which is desirable for dryetching patterns into the substrate.

The structures described so far are of simple geometry. However, the structures that are required in this thesis are more complex and intricate. This means more work is required to try to determine the exposure parameters that give the right response to properly develop a PhQ structure.

Figure 4.11 shows an evolution of PhQ SEM writing. Image (a) is a computer generated 12 -fold $50 \%$ fill factor PhQ structure that was converted for writing. Images (b) to (d) are pictures of the first PhQ structure written at Carleton University. The structure was very large, about $80 \mu \mathrm{m}$ by $80 \mu \mathrm{m}$. The feature lengths are too large for light in the $1550 \mathrm{~nm}$ range. Image (e) shows the first attempt to shrink this structure and the resulting distortions due to the proximity effect. The whole pattern was effectively washed away because the exposure dose was too large. Image (f) had a relatively reduced dose when compared with (e) but was still too high, while image (g) was not high enough to wash the material away, but only made the material swell. Image (h) had the best outcome because the exposure parameter was found to be set just right. 


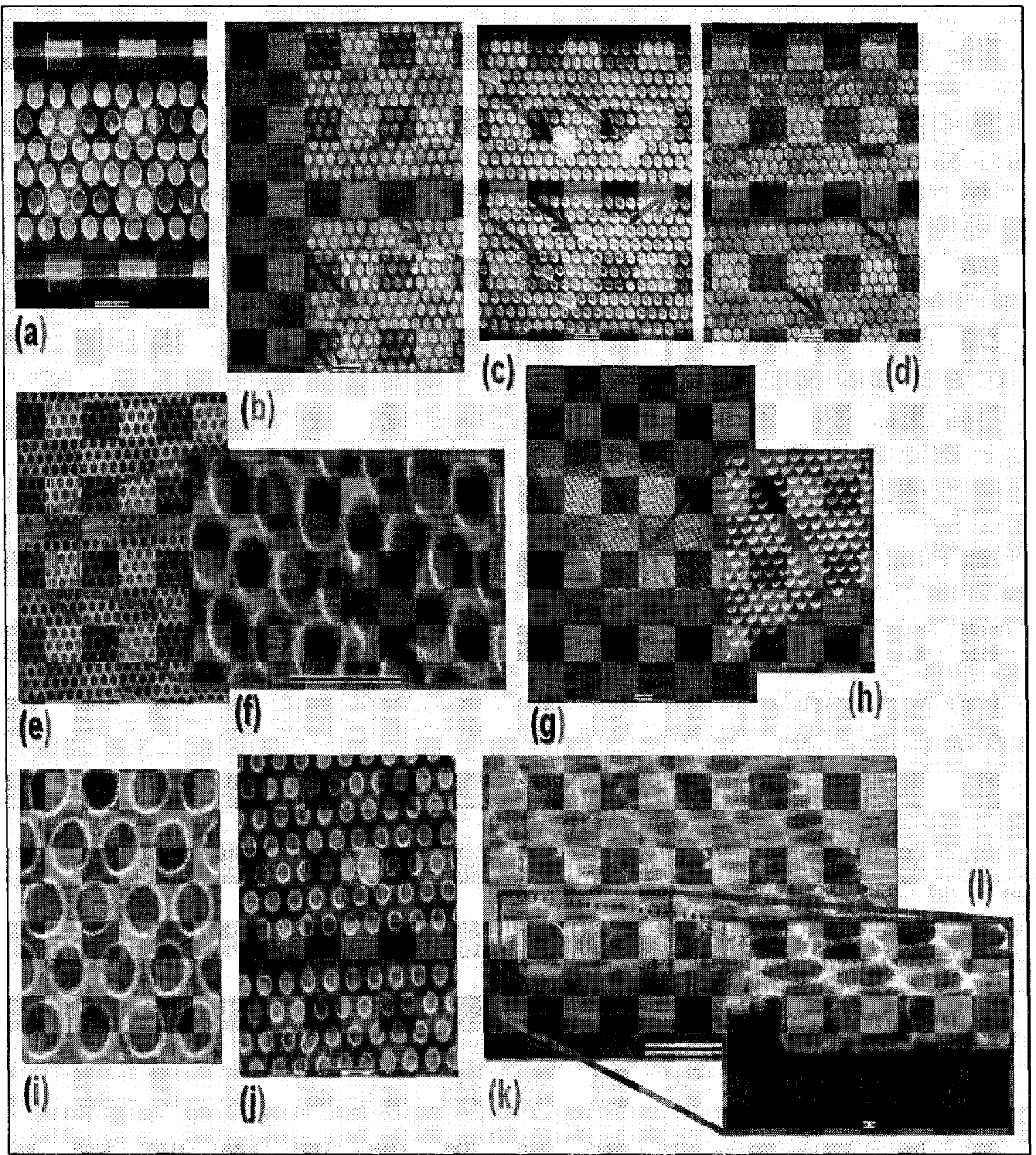

Figure 4.10. Evolution of SEM writing of 2D PhC structures. (a) SEM analysis of figure 4.8(b), (b) (c) and (d) show unexpected defects if writing parameters are not optimized such as proximity effects, (e) better resist structures with intended defect structures, (f) is a close-up of one of the defects - and shows that the resist has developed consistently down to the substrate, $(\mathrm{g})$ and $(\mathrm{h})$ show $2 \mathrm{D} \mathrm{PhC}$ and its close up, (i) shows a close up of the $\mathrm{PhC}$ holes to show curvature, (j) shows other defect structures, and (k) and (l) show a side profile of the resist walls. All bar lengths are one micron except $(\mathrm{g})$ where the length is ten microns. 


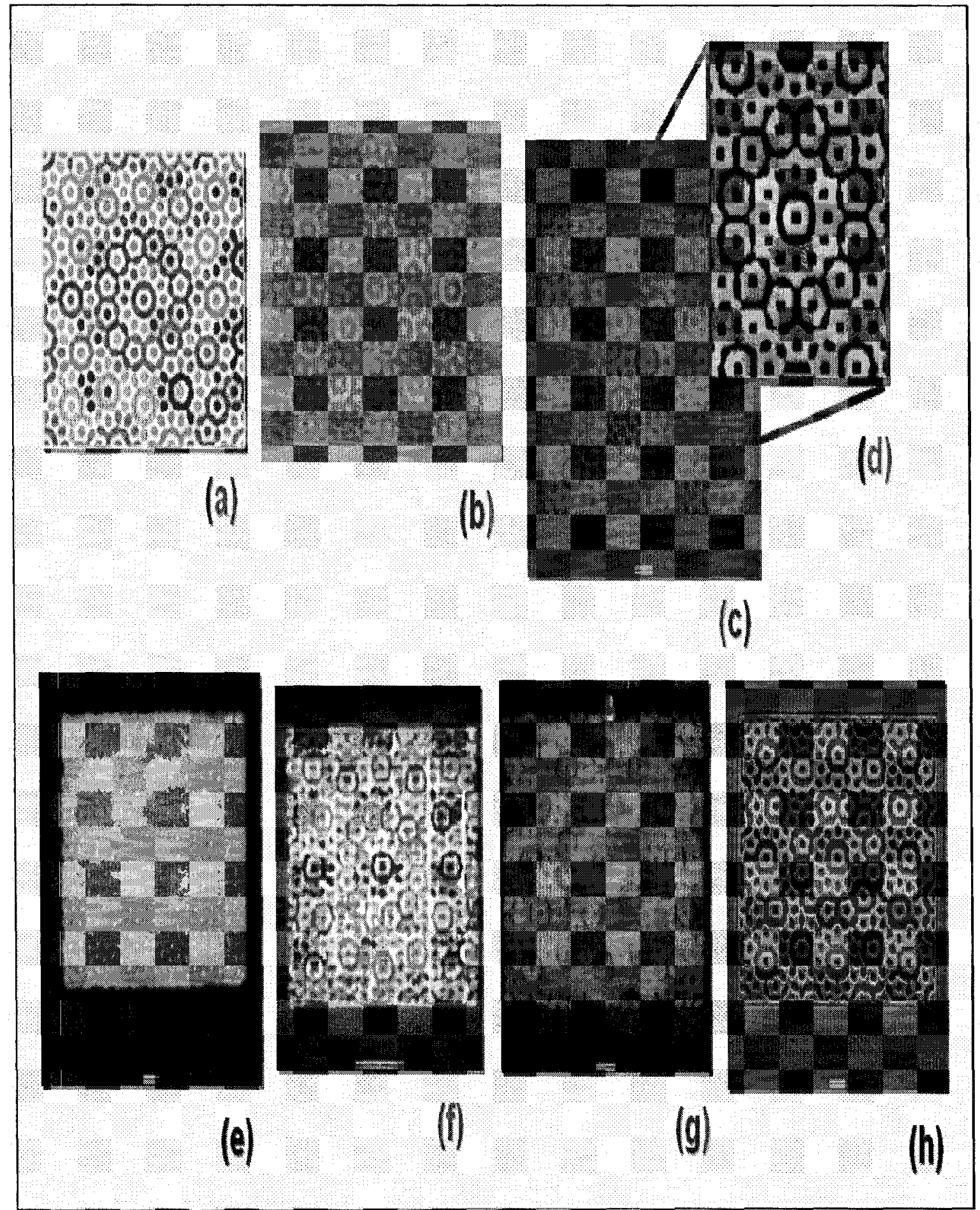

Figure 4.11. $\mathrm{Ph} Q \mathrm{SEM}$ writing evolution. (a) Computer generated image of 12/24 (50\% fill factor) $\mathrm{PhQ}$, (b) optical image of first time ever writing of $\mathrm{PhQ}$ at Carleton University's converted SEM, (c) SEM image of (b), (e), (f), (g) attempts of miniaturizing the $\mathrm{PhQ}$ so that its features are comparable with $1550 \mathrm{~nm}$ light, (h) perfected $\mathrm{PhQ}$ structure with the right exposure parameters. All bar lengths are one micron except (c) where the length is ten microns. 
Figure 4.12 shows that, since one can write a proper $\mathrm{PhQ}$ structure in resist, one can then add line defects and point defects to the write-file and then bring these into the final structure.

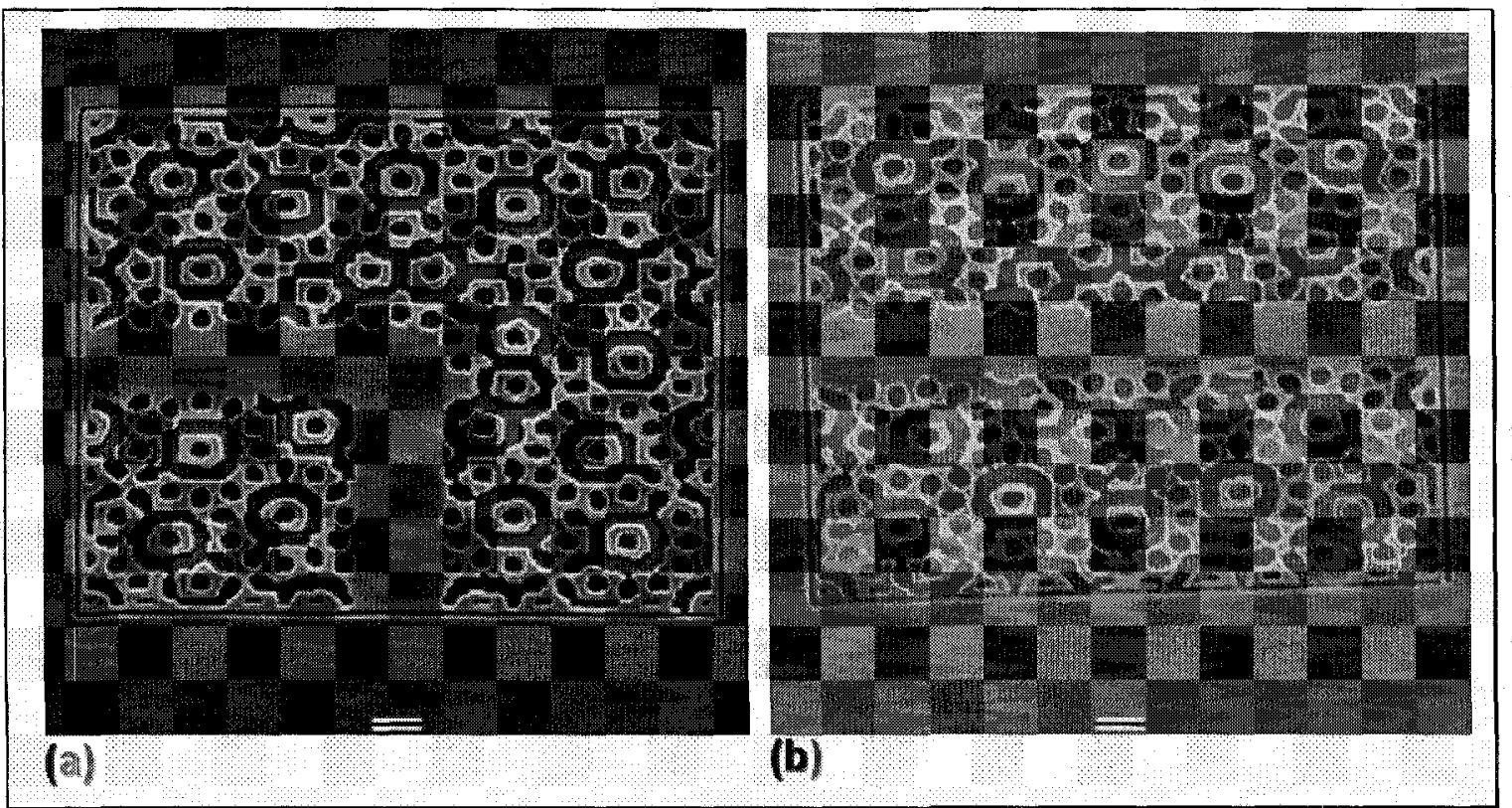

Figure 4.12. Defects can be added computationally and then written with the rest of the structure. (a) Shows a bent waveguide defect in the $\mathrm{PhQ}$ structure, while (b) shows a line and a point defect structure (point defect indicated by arrow). Bar length is one micron.

Chapter 3 demonstrated, via computer simulations, that the PhQ structure that had the most interesting features was the one that had the $20 \%$ fill factor. Figure 4.13 shows the EBL result of this PhQ. Figure 4.13(a) is $10 \mu \mathrm{m}$ by $10 \mu \mathrm{m}$, and as can be seen from the image, the edges are distorted. The central part, image (b), is very close to the computer-generated image. The feature sizes obtained were too small when starting with the desired computer generated image file. The central rod needs to be $250 \mathrm{~nm}$ while in the image itself it is closer to $200 \mathrm{~nm}$. 


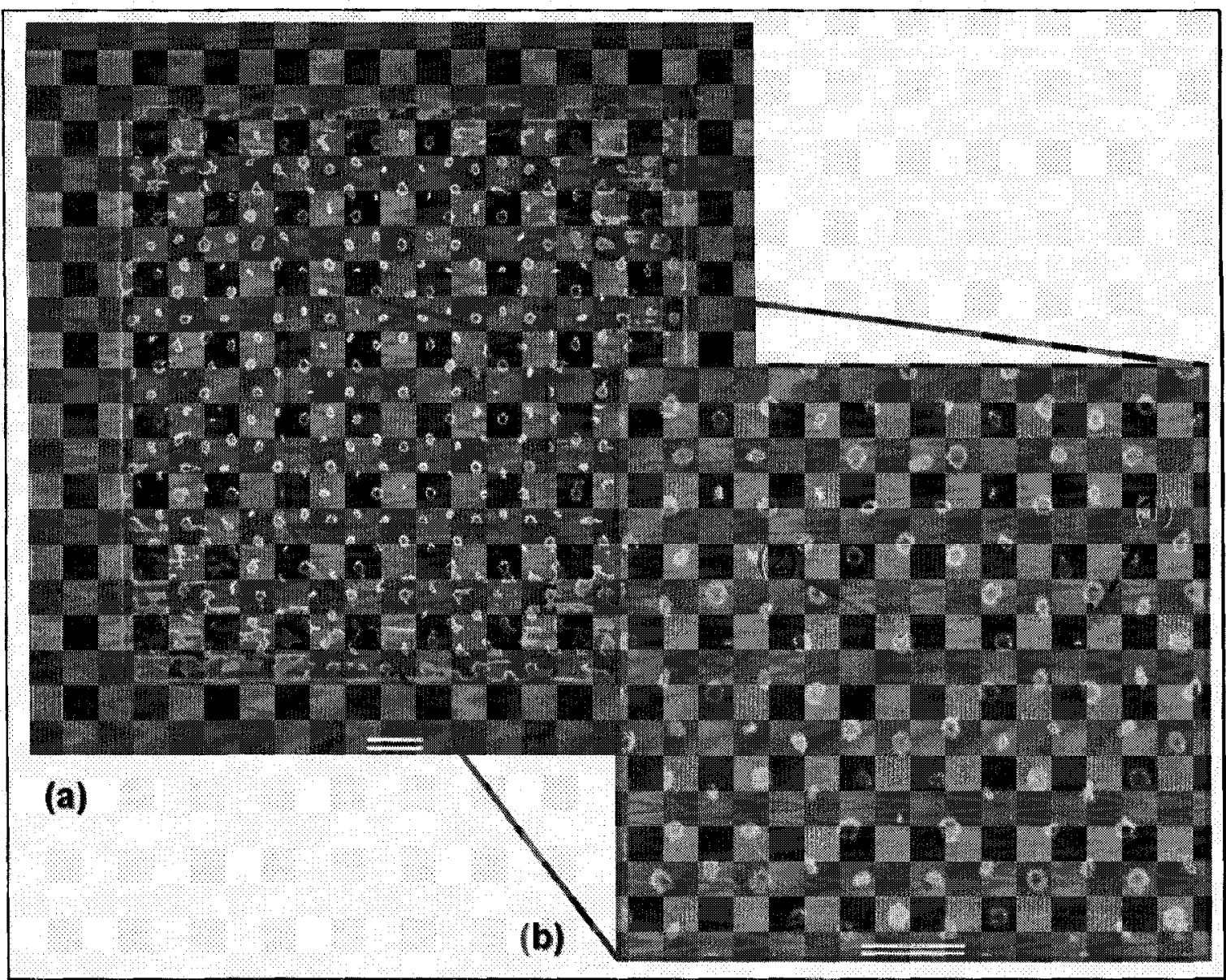

Figure 4.13. (a) A $10 \mu \mathrm{m}$ by $10 \mu \mathrm{m}$ PhQ structure and (b) a close up of the central region. The outer edges are badly developed while the central region is very close to being exactly like the computer-generated image. However, as (1) and (2) in (b) show, because of development, there is shrinkage and the posts are much smaller than they need to be. (1) has a diameter of $200 \mathrm{~nm}$, where it was supposed to be $250 \mathrm{~nm}$ and (2) has a length of $70 \mathrm{~nm}$ where it was supposed to be $120 \mathrm{~nm}$. These numbers are within range of the resolution capability of ZEP (which is $10 \mathrm{~nm}$ for 1 line). Bar length is one micron.

These feature sizes are pushing the limits of the resist, even though its resolution is 10 $\mathrm{nm}$, this is quoted for drawing a line. This is because these structures are 2D patterns and to make such a pattern, one needs to make compensations for proximity effects. Since the development process (and also the etching process) will shrink intended post sizes (and swell intended hole sizes, if one is making $\mathrm{PhCs}$ ) to the pattern to be written, it needs to be altered slightly by the image processing software. It turns out that this 
process helps compensate for development and etching shrinkage. Figure 4.14 shows this process visually.

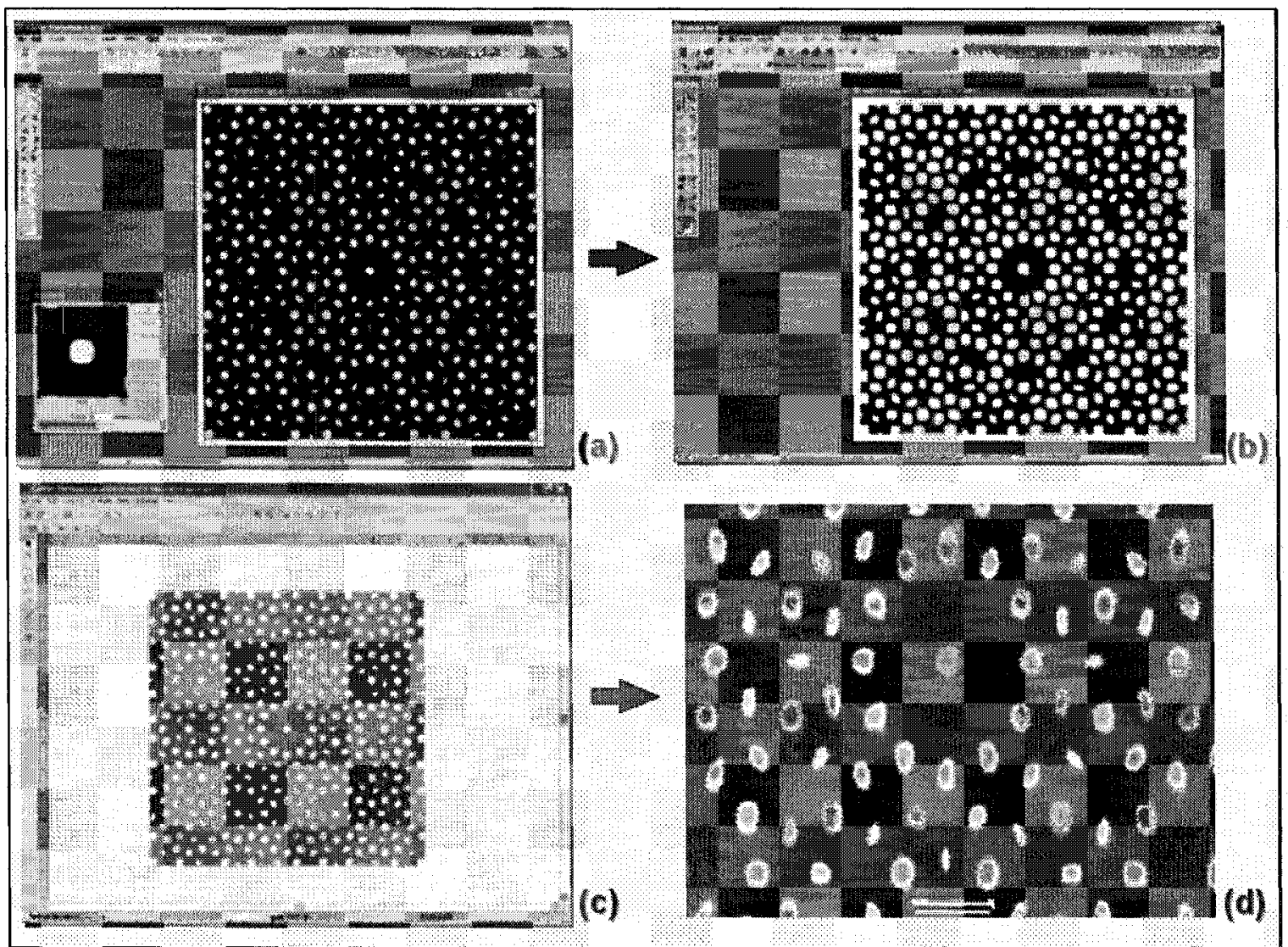

Figure 4.14. Compensation process: (a) choose a filter selection that enlarges white regions, (b) application of swelling feature, (c) transition to CIF for EBL, and (d) fabricated PhQ with the proper diameter middle rod size of $250 \mathrm{~nm}$. Bar length is one micron.

Image (a) is the original PhQ pattern. Using the image processing software, all the white regions in image (a) are enlarged, which yields image (b). This is now the pattern to be written and converted to CIF format, as shown in image (c).

Now that the EBL process was described for writing the PhQs that where studied in Chapter 3, the next process is to etch these patterns into an activate material for measurement. 


\subsection{Dry Etching}

At Carleton University's MFL, the controlled, anisotropic etching of silicon is done with the PlasmaTherm® ${ }^{\circledR}$ SLR-772 Electron Cyclotron Resonance (ECR) Reactive Ion Etcher (RIE). The system is shown in figure 4.15 .

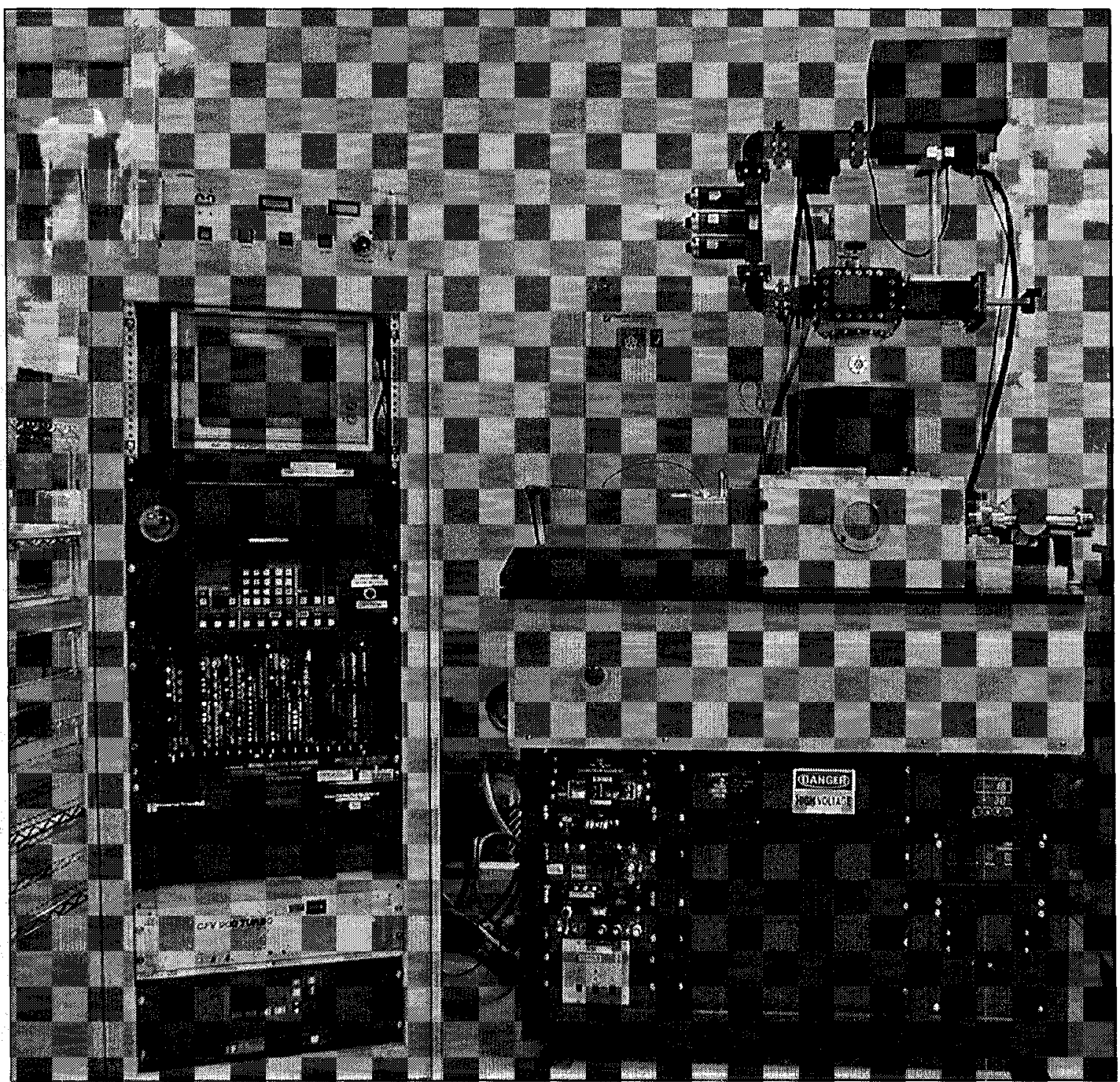

Figure 4.15. PlasmaTherm® SLR-772 Electron Cyclotron Resonance (ECR) Reactive Ion Etcher (RIE) System. Control module is on the left while the actual system in on the right. [Courtesy of Chris Raum]

This ECR system allows for the independent control of plasma excitation power, which is provided with a $700 \mathrm{~W} 2.45 \mathrm{GHz}$ microwave source, and ion bombardment energy at the 
substrate controlled by a separate RF bias source. The system is pumped down to controlled pressures by an Alcatel $5900 \mathrm{CP}$ turbo pump. The system had been originally tuned to be a dedicated polysilicon gate etcher. It is controlled by an elaborate gamut of switches, shown in figure 4.16 , that only authorized users are allowed to operate. The author was granted this authority and became very familiar with this machine over time.

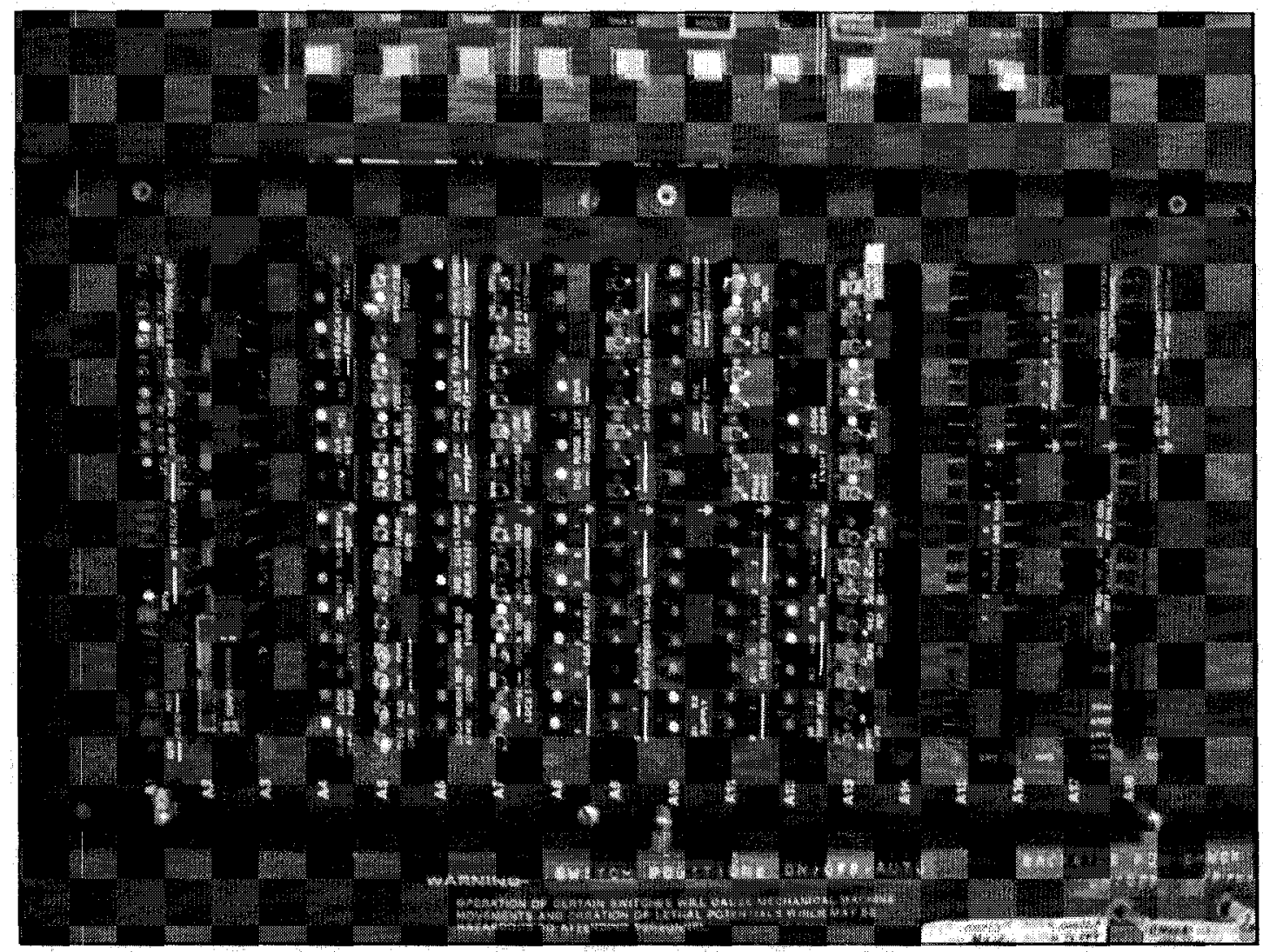

Figure 4.16. Close up of control module for the ECR. [Courtesy of Kristen Medri]

Due to the MFL now being used to fabricate integrated photonic devices, etching parameters must be tuned for etching $\mathrm{PhCs}, \mathrm{PhQs}$ and waveguides. In this section, these parameters, along with etching results will be presented. 


\subsubsection{Plasma Review}

Metals are described as a lattice of ions in an electron bath. Imagine now that whatever holds this lattice together is suddenly gone and the ions float about in a random direction: this is a plasma. Plasmas are generated by the rapid dissociation of gases by high energy electric and magnetic fields. The result is an energized salad of neutrals, ions, photons, electrons, and highly reactive radicals. A surface exposed to a plasma will experience a flux of particles resulting in bombardment, stimulating the production of outgoing fluxes. This is the etching mechanism.

Plasmas are generated from the electrical breakdown of neutral gases. In the case of RIE, the gas is in between an anode and cathode, and the electrical energy is either provided by a direct voltage or an RF source. The free electrons collide with the neutral molecules which results in ionization of the gas. Because the electrons are much lighter than the ions, they move around a lot faster and this leads to an avalanche effect. The plasma structure becomes more elaborate because of the dynamics of the particles involved - with different regions having different properties and names.

By controlling the plasma generation process, one can improve plasma qualities. The goal is to generate a high-density plasma (which increases etch rates) in a low pressure environment (which increases anisotropy) and control the energy of the ions so that it is uniform and low enough not to cause physical damage to other features, and high enough to react with the material that is being etched

This topic is called Deep Reactive Ion Etching (DRIE) and there are two main techniques for controlling the plasma generation: Inductively Coupled Plasma (ICP) and Electron Cyclotron Resonance (ECR). The ICP is common but will not be described in 
detail here. Essentially, it is a helical coil that has a time-varying electric current passing through it and it creates a time-varying magnetic field around it, which induces azimuthal electric currents in a neutral gas, leading to breakdown.

\subsubsection{Electron-Cyclotron Resonance Etching}

The ECR method is used at Carleton University. This method exploits the Lorentz force applied to electrons in a magnetic field. Once the angular frequency of the electron is established by the magnetic field, a microwave source matches this frequency and gives energy to the electrons. This causes a higher density plasma. Figure 4.17 shows a detailed description of ECR Method. Image (a) is the top portion of the right hand side of figure 4.15. Item (1) in image (a) is the microwave source and item (2) depicts the tuning stubs. Image (b) is a schematic of the ECR system, and the arrows from (a) to (b) connect the related regions in the physical picture to the schematic view.

The chamber of the ECR, where the sample sits, is cryogenically cooled to increase etch anisotropy. With the ECR system described, the next step is to explain how to etch the EBL written PhQ structures. 


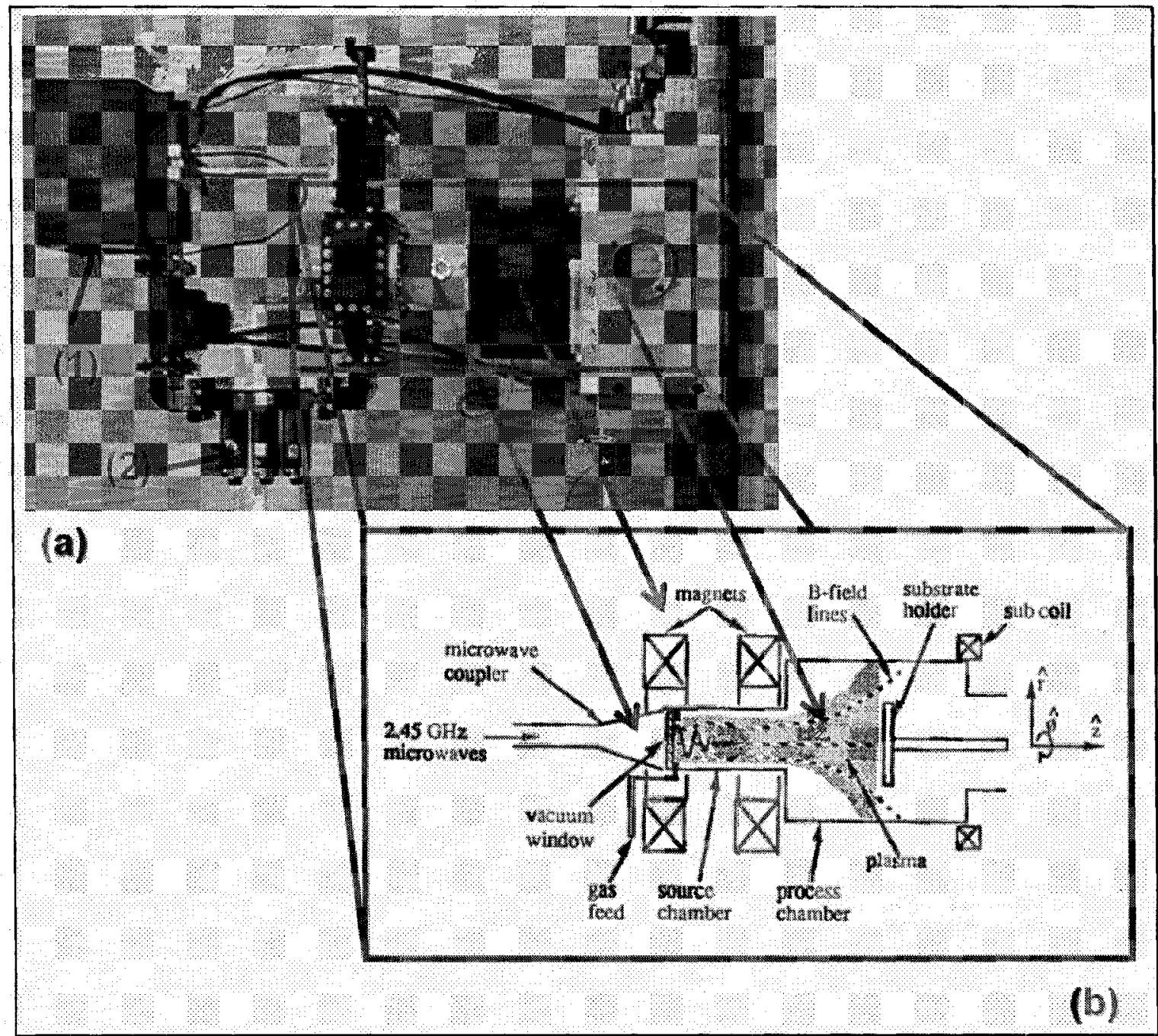

Figure 4.17. Detailed description of ECR Method. (a) the top portion of the of the right hand side of figure 4.15. Item (1) is the microwave source and (2) depicts the tuning stubs. (b) is a schematic of the ECR system and the arrows from (a) to (b) connect the related regions in the physical picture and the schematic view.

\subsubsection{Dry-Etching of Structures}

The ECR etcher is designed to accept only 4 inch wafers. This means that the sample piece that has the $\mathrm{PhQ}$ pattern needs to be put on a 4 inch backing wafer. In the past, photoresist was used as an adhesive to keep the sample on the backing wafer. However, the baked resist had poor heat conductivity, which resulted in a more isotropic etch. Carbon tape is now used instead of photoresist and it demonstrates better heat conductivity, resulting in a more anisotropic etch. After the etching is done, the backing 
wafer is placed on a cooled hotplate, and then the hotplate is turned on. Immediately after the hotplate is turned on, a flatheaded tweezer is gently placed at the edge of the sample and rocked slowly. The heat will loosen the carbon tape and at the right moment, the tweezers can lift the sample off the backing wafer while the carbon tape remains on the backing wafer. If it is not the right moment, one will either break the sample (if done too soon) or the carbon tape will stick to the bottom of the sample (if not done soon enough). Removing the carbon tape from the sample can be very messy and can potentially even ruin the sample.

As noted in the EBL section, if the pattern to be realized is not compensated (as shown in figure 4.14) for development and etching, the posts will be much smaller. In addition to this, if the etching parameters are not optimal, the pattern will not be uniform, as shown in figure 4.18. The graininess most likely comes from grass residue, which stems from sputtered silicon from silylated areas. A higher plasma density can help with this, so the ECR was tuned to give a higher plasma density. 


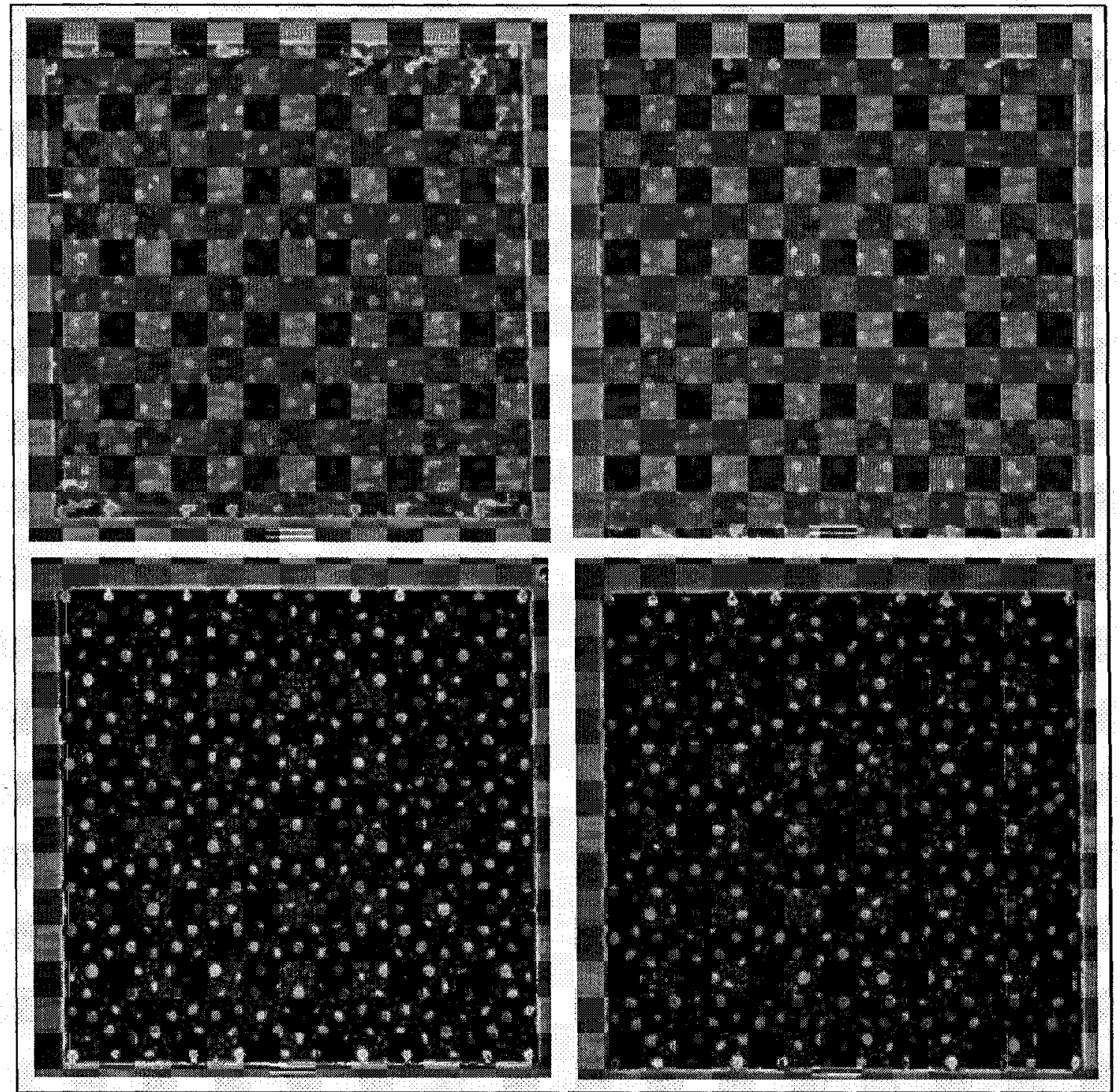

Figure 4.18. Four etched PhQ structures of the same PhQ pattern. The substrate looks grainy. This is most likely grass residue. Bar length is one micron.

Figure 4.19 shows the result of higher density plasma from the ECR, which effectively smoothed out the graininess seen in the previous structures. Figure 4.19 shows the PhQ structure with a waveguide across it. This is the structure that was described and simulated in Chapter 3. The waveguide was written in so that it could bring the optical energy to the center of the PhQ pattern so that one could measure the structure's optical response. Because silicon has a high index of refraction and the waveguide mode is highly confined, the coupling from the waveguide to the PhQ pattern 
may not occur. In order to generate coupling, the waveguide sidewalls were perturbed by the $\mathrm{PhQ}$ pattern.

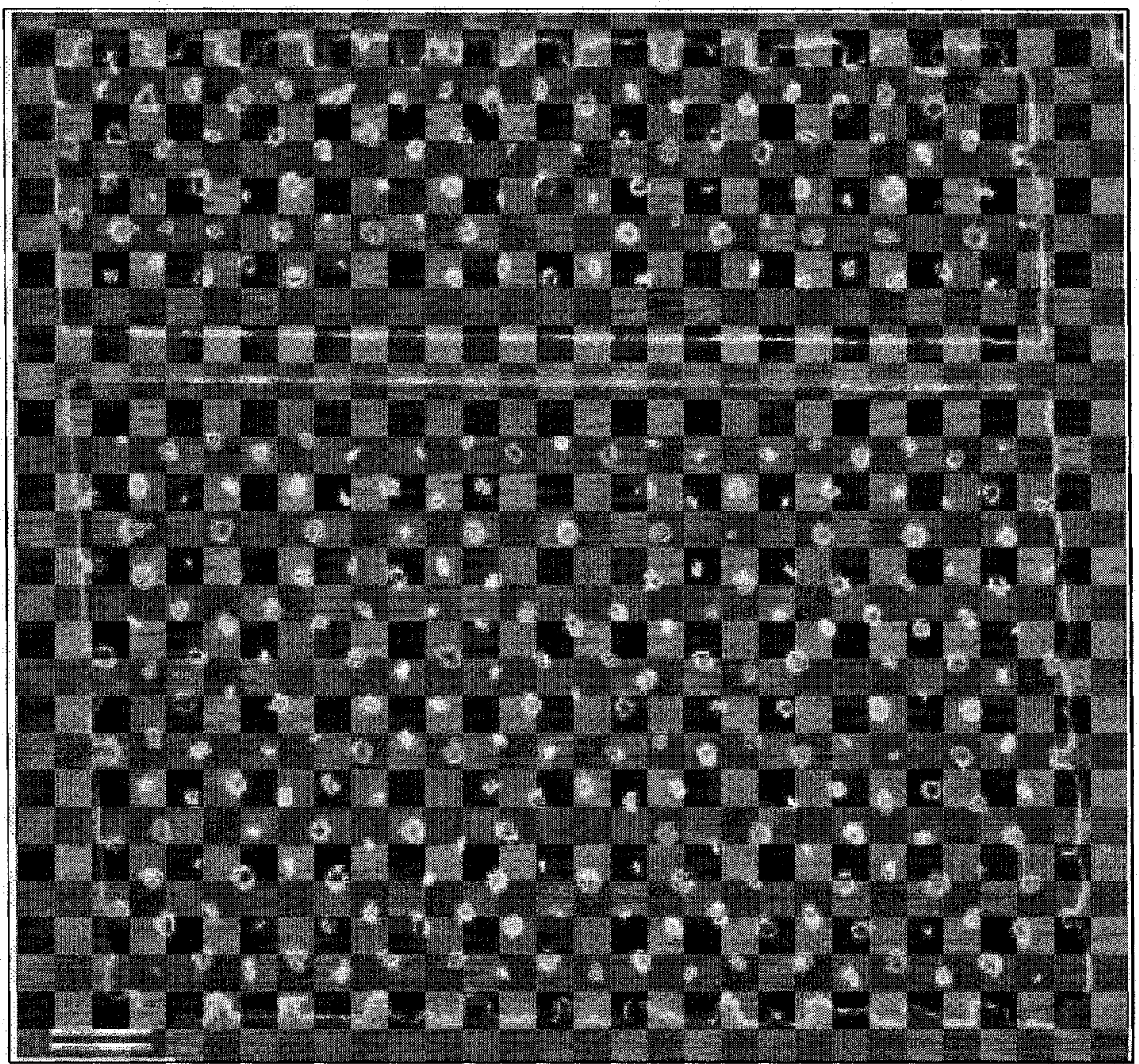

Figure 4.19. Higher density plasma smoothed background. The tilt in the image is an artifact of the SEM viewing system and is not physically there. Bar length is one micron. This is shown in figure 4.20. Notice the right angle structures in the CIF image in figure 4.20. These are the alignment marks for adding the external waveguides. Further detail on combing the EBL patterns with photolithography to add the external waveguides will be discussed later. 


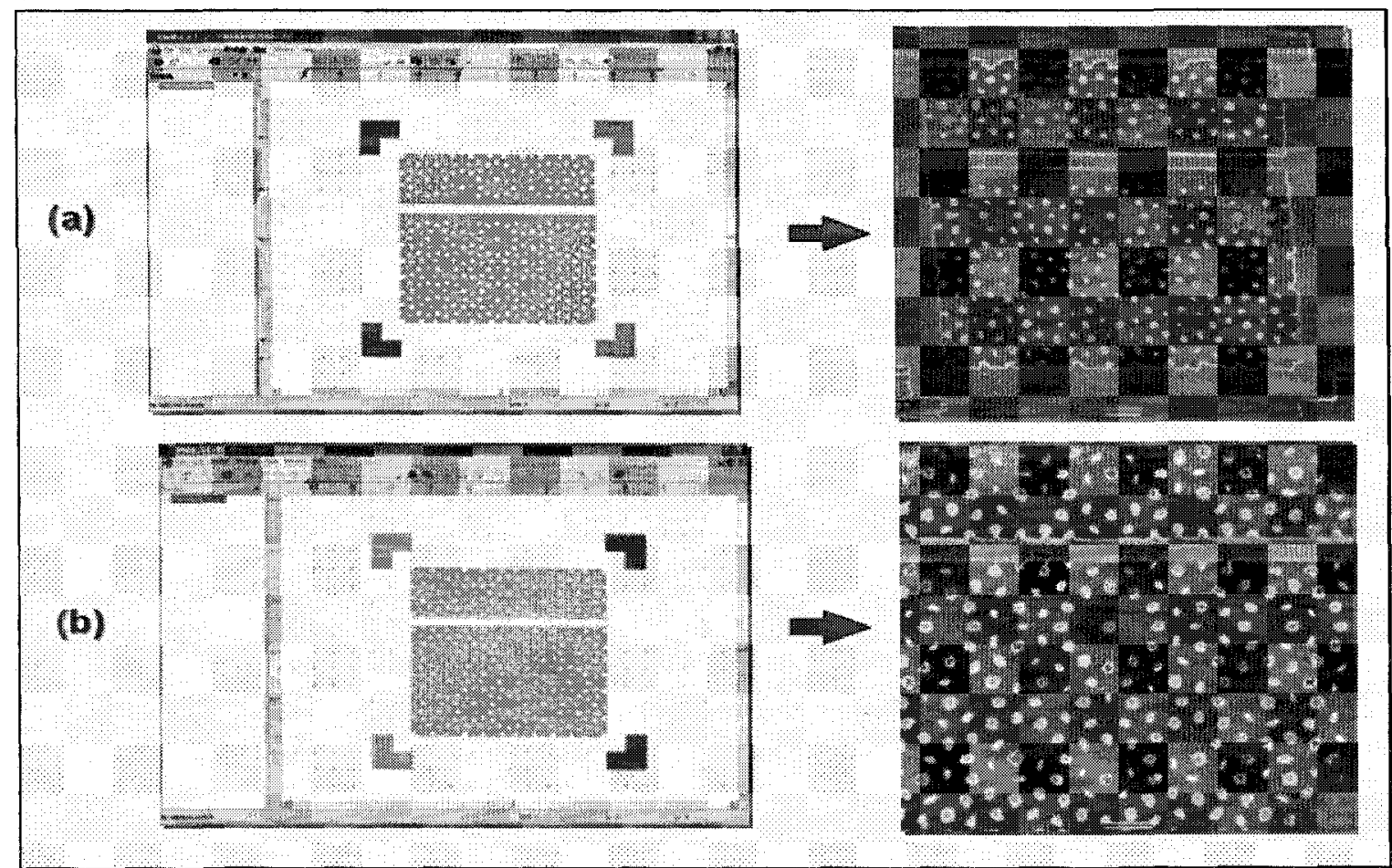

Figure 4.20. Going from unperturbed waveguide to perturbed waveguide to increase scattering. (a) Unperturbed waveguide-PhQ pattern. (b) Perturbed waveguide-PhQ pattern. Bar length is one micron.

Before adding the external waveguides, it would be good to assess if the rods of the pattern are indeed rods or are only just mounds. Careful study of etch parameters by the author yielded posts (as indicated in figure 4.21), although the edges are rough. Hopefully future work can smooth out this roughness.

One more EBL pattern will be described that aids in increasing the efficiency of delivering light to the PhQ structures. These structures are known as J-couplers. 


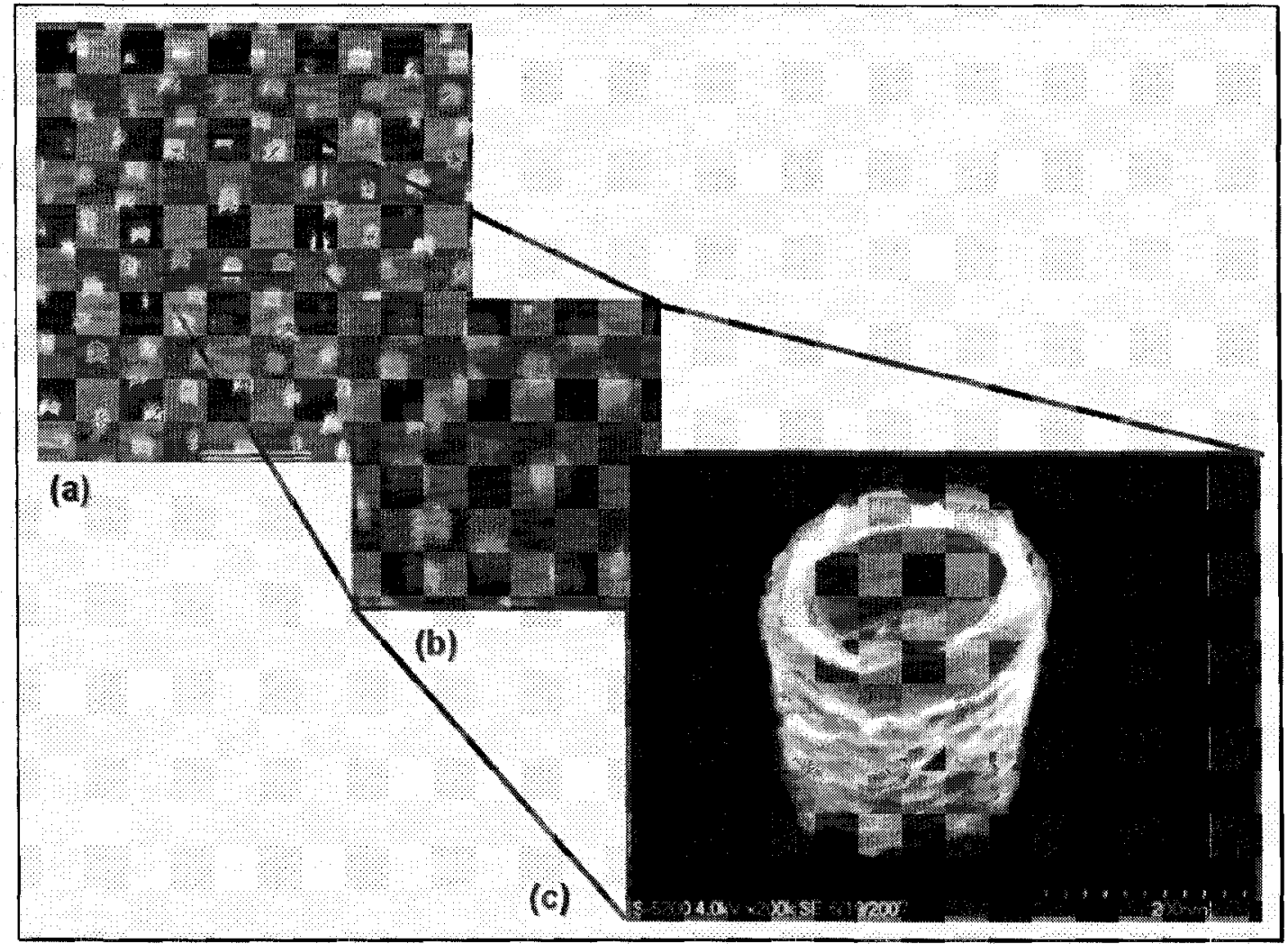

Figure 4.21. Post analysis. (a) Tilted view of large area of PhQ. (b) Closer view. (c) Extreme close-up of post. All bar length are one micron except (c) where the length is $200 \mathrm{~nm}$.

\subsection{Accessing PhQ Patterns for Measurement}

The converted SEM is limited to at most $100 \mu \mathrm{m}^{2}$ of writeable area. Such a small area is difficult, if not impossible, to work with for measurement purposes. By writing alignment marks with the PhQ patterns, one can then use photolithography to overlay a waveguide mask and then expose waveguides to gain access to the PhQ pattern. In the waveguide mask used for this work, there were no tapered waveguides. Tapering the waveguide is important to increase coupling efficiencies, but in this case maximal scattering was required to at least show that the CAM states of the PhQ pattern exist. Having the capability to efficiently couple into the PhQ for some experiments is important. Purchasing a mask with tapered waveguides can be quite costly, especially for 
waveguide diameters below $1 \mu \mathrm{m}$. In addition to this, the photolithography mask aligner at Carleton is poorly suited to resolve feature sizes below $1 \mu \mathrm{m}$.

Instead of purchasing costly masks to use on an offsite photolithography system that can resolve the features, the author developed an EBL tapering method based on Jcouplers [53].

\subsubsection{J-Coupler Design}

The J-Coupler design exploits the geometry of a parabola to perform the equivalent of a taper-like architecture. A portion of a carefully designed parabola is used as a mirror to reflect light to a focused spot on the waveguide. Figure 4.22 shows the J-coupler geometry used for analysis and design.

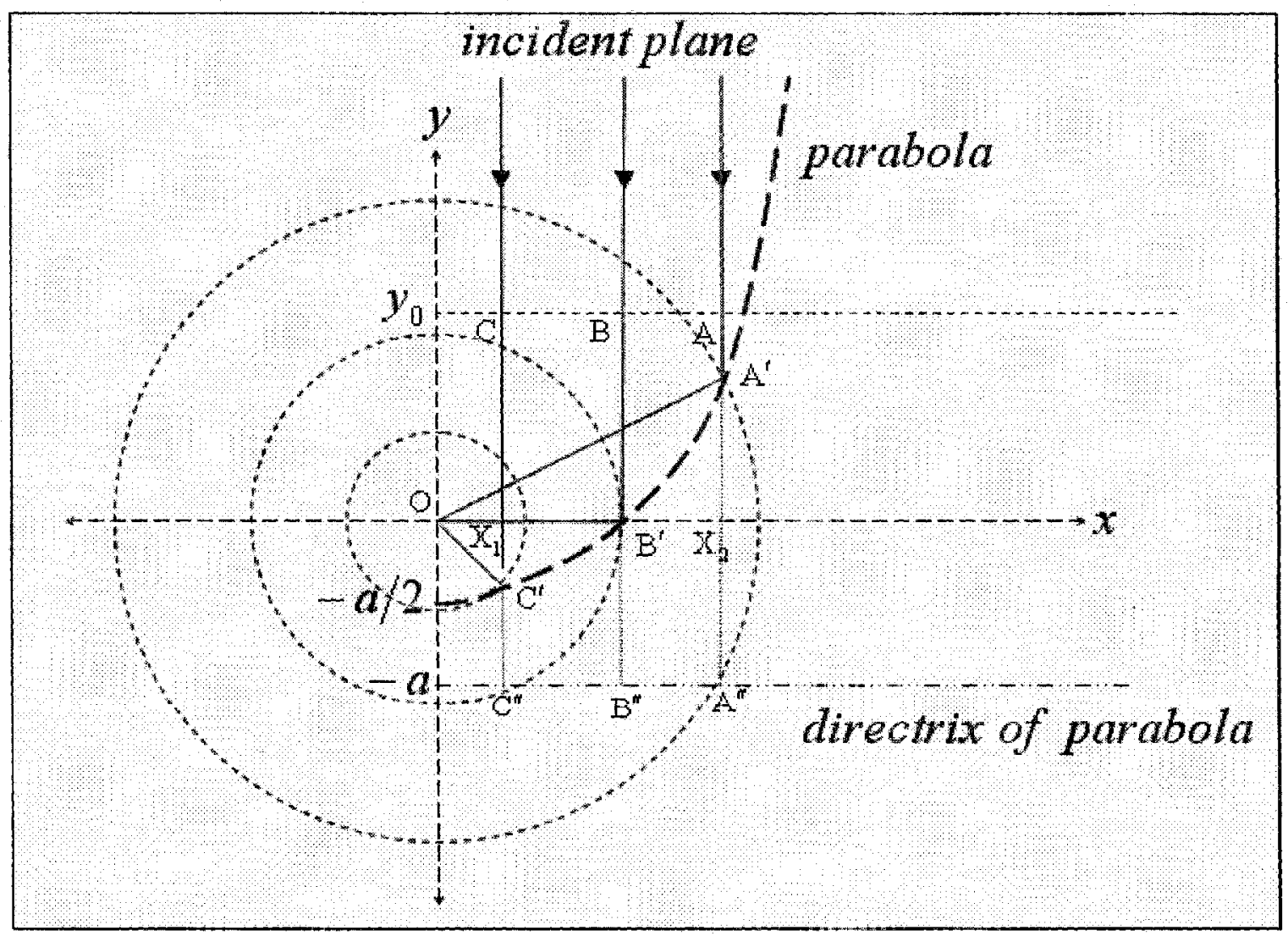

Figure 4.22. Illustration of $J$-coupler geometry used for analysis and design. 
The focal point is chosen to be the origin. The incident planewave is chosen to propagate in the negative $y$ direction. In order for the wave to be focused at the origin, the following condition must be met for each ray as it reflects from the parabola surface, represented by the thick dashed line in figure 4.22 ,

$$
y_{0}-y+\sqrt{x^{2}+y^{2}}=\text { constant }
$$

where $y_{0}-y \equiv \mathrm{AA}^{\prime} \equiv \mathrm{BB}^{\prime} \equiv \mathrm{CC}^{\prime}$ and $\sqrt{x^{2}+y^{2}} \equiv \mathrm{A}^{\prime} \mathrm{O} \equiv \mathrm{B}^{\prime} \mathrm{O} \equiv \mathrm{C}^{\prime} \mathrm{O}$ in figure 4.22. A curved mirror shape that fulfills this condition is a parabola with a directrix shown in figure 4.22, where $A^{\prime} A^{\prime \prime}=A^{\prime} O, B^{\prime} B^{\prime \prime}=B^{\prime} O$ and $C^{\prime} C^{\prime \prime}=C^{\prime} O$. These equalities imply,

$$
\sqrt{x^{2}+y^{2}}=a+y
$$

so that,

$$
x^{2}+y^{2}=a^{2}+2 a y+y^{2} \Rightarrow y=\frac{x^{2}}{2 a}-\frac{a}{2},
$$

which is the equation of the parabola.

In order for the light to be directed completely into the $x$-direction at the origin, all $y$-components to the rays must be zero at the origin. The $y$-components of all the rays can be obtained from the slope of the tangent at each reflection point on the parabola and the slope of each outgoing ray.

Generally, if $f(x)$ describes a mirror function, then $f^{\prime}(x)$ is the slope of the tangent at $x$. Figure 4.23 shows the mirror function, the slope at the reflection point of the mirror function and the slope of the outgoing ray from a vertical incoming ray. 




Figure 4.23. Slopes required to find $y$-components of outgoing rays.

The formula for the outgoing ray slope can be determined via trigonometry and the knowledge that the angle of incidence is equal to the angle of reflection. At this point, the two slopes are equal,

$$
y=\frac{f^{\prime}(x)^{2}-1}{2 f^{\prime}} x=f^{\prime}(x) x+b \Rightarrow y=\frac{1-f^{\prime}(x)^{2}}{1+f^{\prime}(x)^{2}} b,
$$

where is $b$ is the $y$-axis intersection of the tangent. There is no $y$-axis intersection for the outgoing ray because it is directed through the origin. If one makes $b$ equal to 1 , then 
4.19 is a signed measure of $y$-component strength in the outgoing ray. In order for there to be no $y$-component strength at all at the origin, the integral,

$$
\int_{x_{1}}^{\mathrm{x}_{2}} \frac{1-(x / a)^{2}}{1+(x / a)^{2}} w(x) d x
$$

must vanish. In $4.20, f(x)=\frac{x^{2}}{2 a}-\frac{a}{2}, \mathrm{X}_{1}$ and $\mathrm{X}_{2}$ are the $x$-axis coordinates determined by the width of the incoming wave, $w(x)$, where it is a weight function that corresponds to the intensity distribution of the incoming wave. If a planewave is selected, then $w(x)$ is a constant that can be normalized to unity, and 4.20 can be calculated analytically,

$$
\mathrm{X}_{1}-\mathrm{X}_{2}=2 a\left[\tan ^{-1}\left(\frac{\mathrm{X}_{1}}{a}\right)-\tan ^{-1}\left(\frac{\mathrm{X}_{2}}{a}\right)\right] \text {. }
$$

Relation 4.21 is now a constraint of the design of the J-coupler. The difference, $\mathrm{X}_{1}-\mathrm{X}_{2}$, is the width of the incoming beam and waveguide. The author reused an existing waveguide mask design which has a width of $5 \mu \mathrm{m}$. To make things simple, the author chose $X_{1}=-5 \mu \mathrm{m}$ and $X_{2}=-10 \mu \mathrm{m}$ on an $x y$-coordinate system and found the right $a$ to give $5 \mu \mathrm{m}$ on the right-hand side of 4.21 . In this case, $a=7.37 \mu \mathrm{m}$ for the left and right-hand sides of 4.21 to equal.

The J-coupler was designed computationally using COMSOL®. The base parabola is represented by the equation,

$$
y=\frac{x^{2}}{1.474 \times 10^{-5}}-3.685 \times 10^{-6}
$$

Figure 4.24 shows the subsequent stages required to arrive at the J-coupler design. Image (a) shows half of the parabola and the waveguide intersection points are 
determined. Image (b) shows the waveguide and parabola together and (c) shows the results of a $\mathrm{COMSOL} \circledast$ geometric intersection function, yielding the waveguideparabolic mirror section. Image (d) shows the taper section drawn in and intersecting at the parabolic mirror. Note that this taper section traces the ray paths from the associated points on the parabolic section. Image (e) is the resultant J-coupler design after a union function is applied on (d). Image (f) is the complete J-coupler input and output system drawn with the photomask geometries.

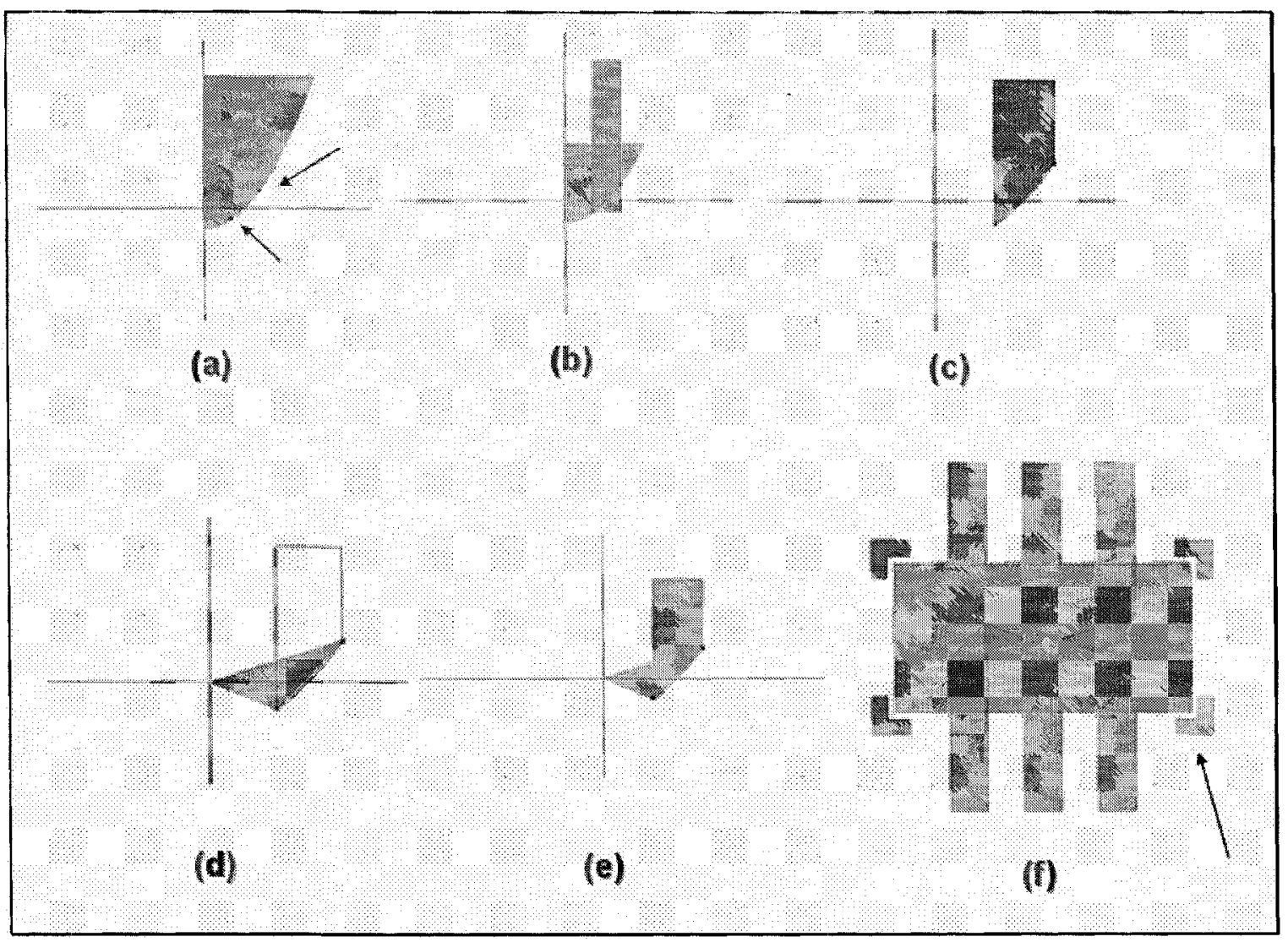

Figure 4.24. J-Coupler design stages. (a) Base parabola halved and waveguide intersection points identified. (b) Waveguide region added. (c) Intersection function used to generatc parabolic section of waveguide. (d) Taper region drawn. (e) Union function used to unite taper and parabolic region. (f) J-couplers overlaid with photomask design to insure J-couplers have appropriate dimensions. PhQ patterns would then be written between the J-coupler regions.

Once the J-coupler system is complete, it can then be transferred over to the EBL system for writing and then the pattern can be etched. Figure 4.25 shows three fabricated 
structures with J-couplers present. Image (a) is a J-coupler system with a $0.5 \mu \mathrm{m}$ width photonic silicon wire, (b) is a J-coupler system and a $\mathrm{PhC}$ pattern, and (c) is a $\mathrm{J}$-coupler system with a PhQ system.

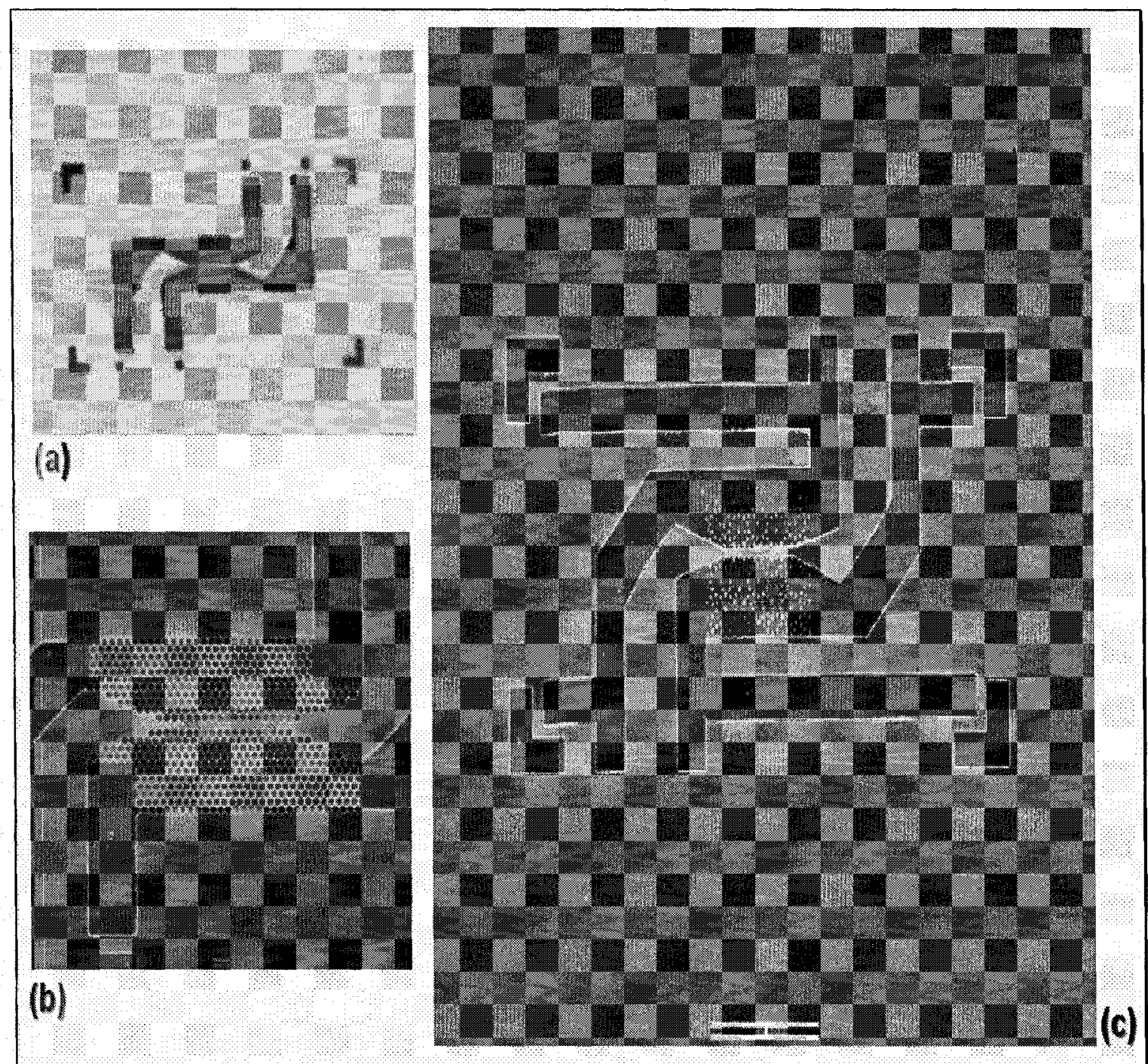

Figure 4.25. Physical fabrication of J-Coupler systems. (a) Optical image of J-coupler with photonic silicon wire. Size of pattern is $46 \mu \mathrm{m}$ by $26 \mu \mathrm{m}$. (b) SEM image of Jcoupler and $\mathrm{PhC}$ system, where bar length is one micron and (c) SEM images of Jcoupler and PhQ system, here bar length is $10 \mu \mathrm{m}$.

EBL of J-coupler systems with smaller and complicated patterns need special attention. The NPGS software is able to detect different layers in a CIF file and can accordingly change exposure parameters for each layer. Figure 4.26 shows an image of 
the CIF drawing for a J-coupler-PhC system. The J-coupler is one color while the $\mathrm{PhC}$ is of another color.

The next section will discuss photolithography of a waveguide mask for access to the PhQ and J-coupler systems.

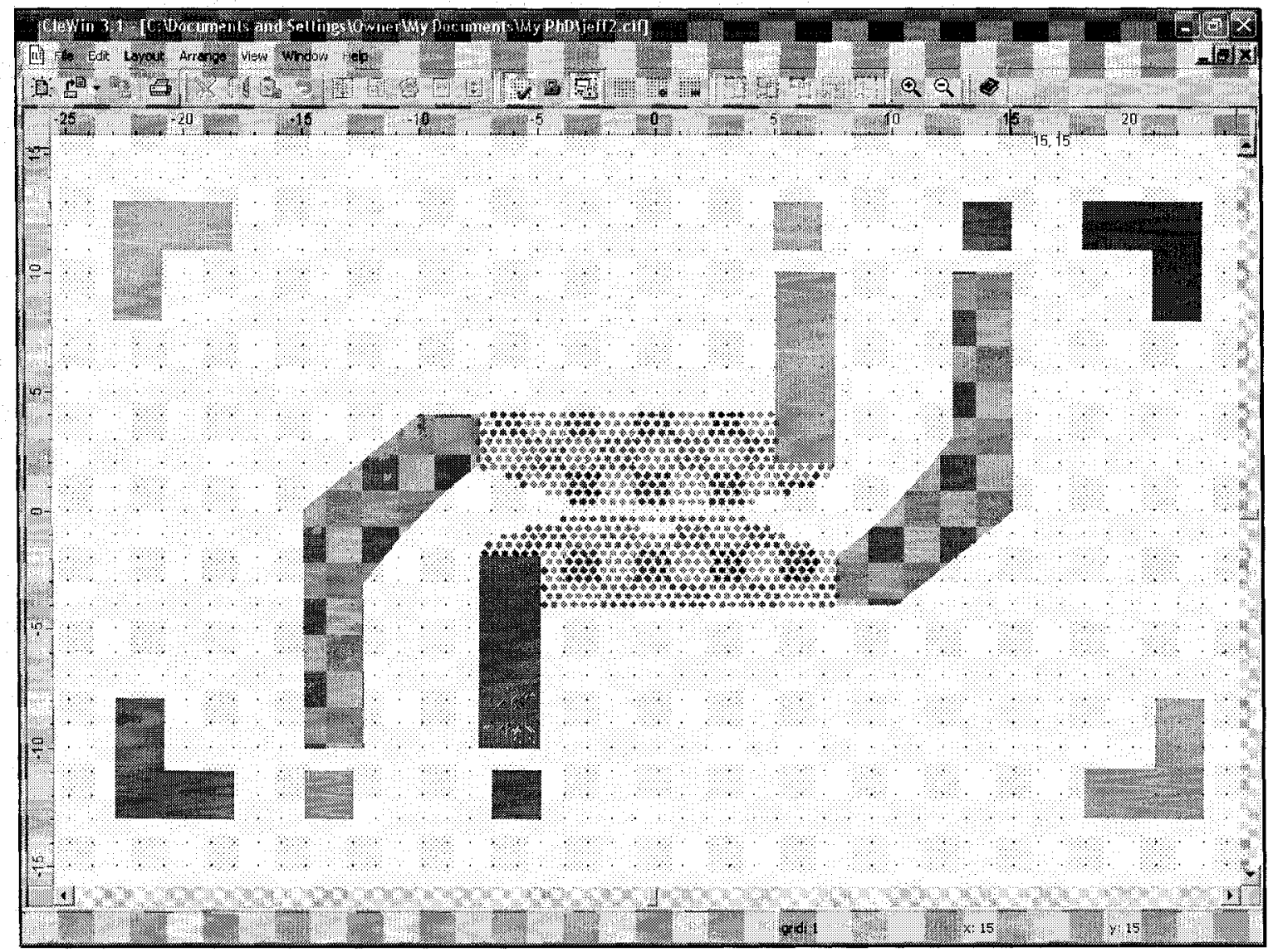

Figure 4.26. CIF Editor image of J-Coupler-PhC system with different layers shown.

\subsubsection{Waveguide Aligning}

In order to add the external waveguides, the ZEP is stripped away using a high oxygen content plasma etcher. Then photoresist, Shipley's® $\mathrm{S} 1811$, is spun on the sample at $4000 \mathrm{rpm}$ for 30 seconds. The mask containing the waveguides is loaded onto the mask aligner along with the sample. The mask is then aligned with the alignment marks drawn by the EBL. Figure 4.27 shows an example of this process. Here the J-coupler EBL 
pattern requires three waveguides coming in from the left and three waveguides going out to the right. This is similar to the configuration shown in image (f) of figure 4.24.

It shows an unaligned EBL J-coupler-PhC pattern, unaligned photolithography mask and an aligned EBL J-coupler-PhC pattern with the photolithography mask. 


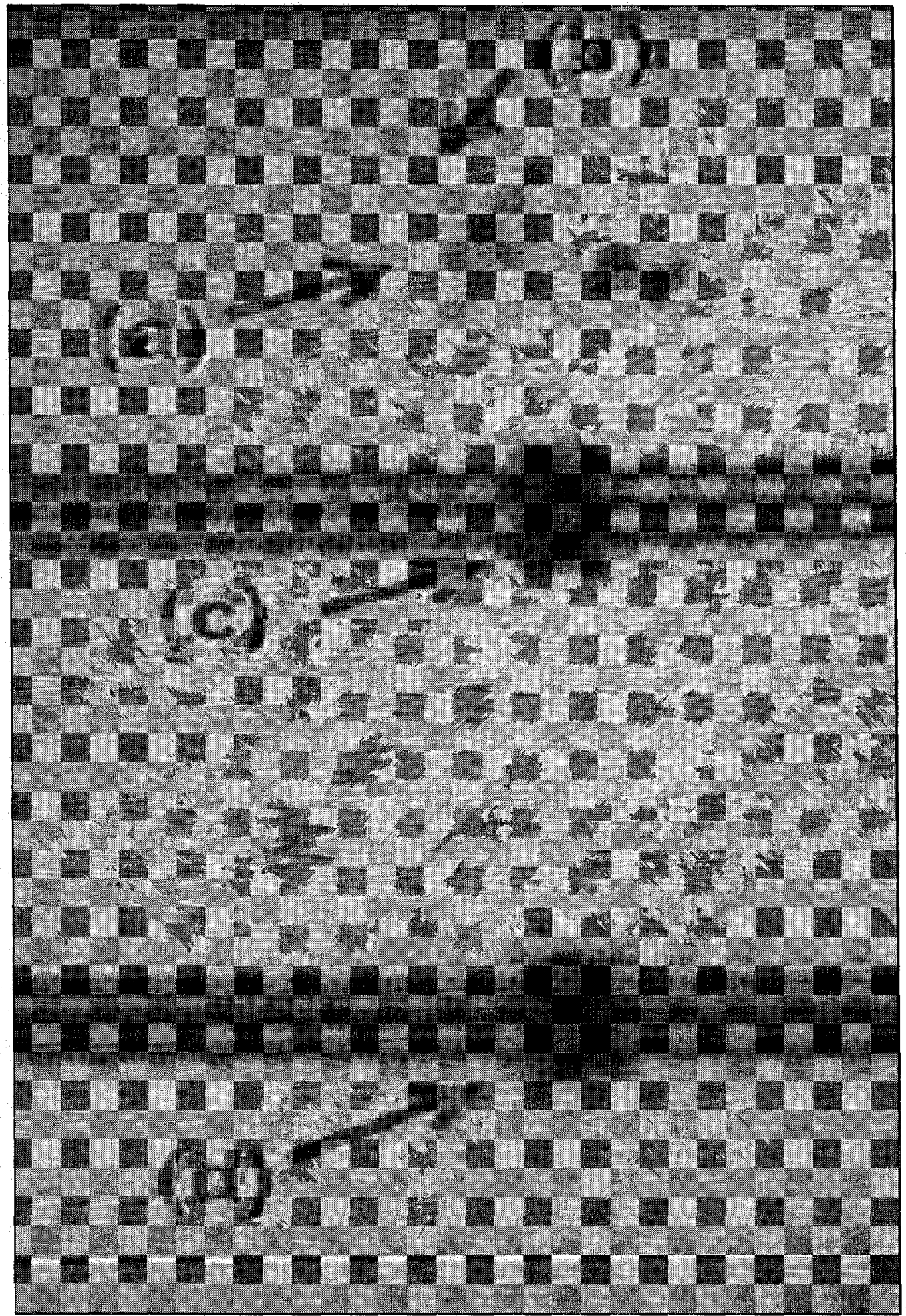

Figure 4.27. Masking aligning with EBL pattern. (a) Unaligned EBL Jcoupler-PhC pattern. This pattern is $26 \mu \mathrm{m}$ across and $46 \mu \mathrm{m}$ down. (b) Alignment mark written by electron-beam. (c) Unaligned photolithography waveguide mask. (d) Aligned EBL pattern to photolithography mask. [Courtesy of Kristen Medri] 


\subsubsection{Fabrication of Three Systems of Observation}

The three systems to build are shown in figure 4.28 as CIF files in the layout editor.

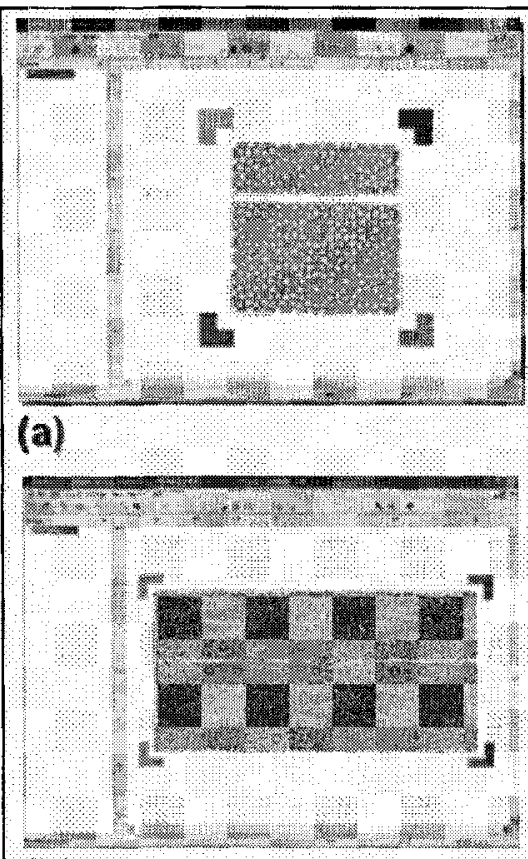

(b)

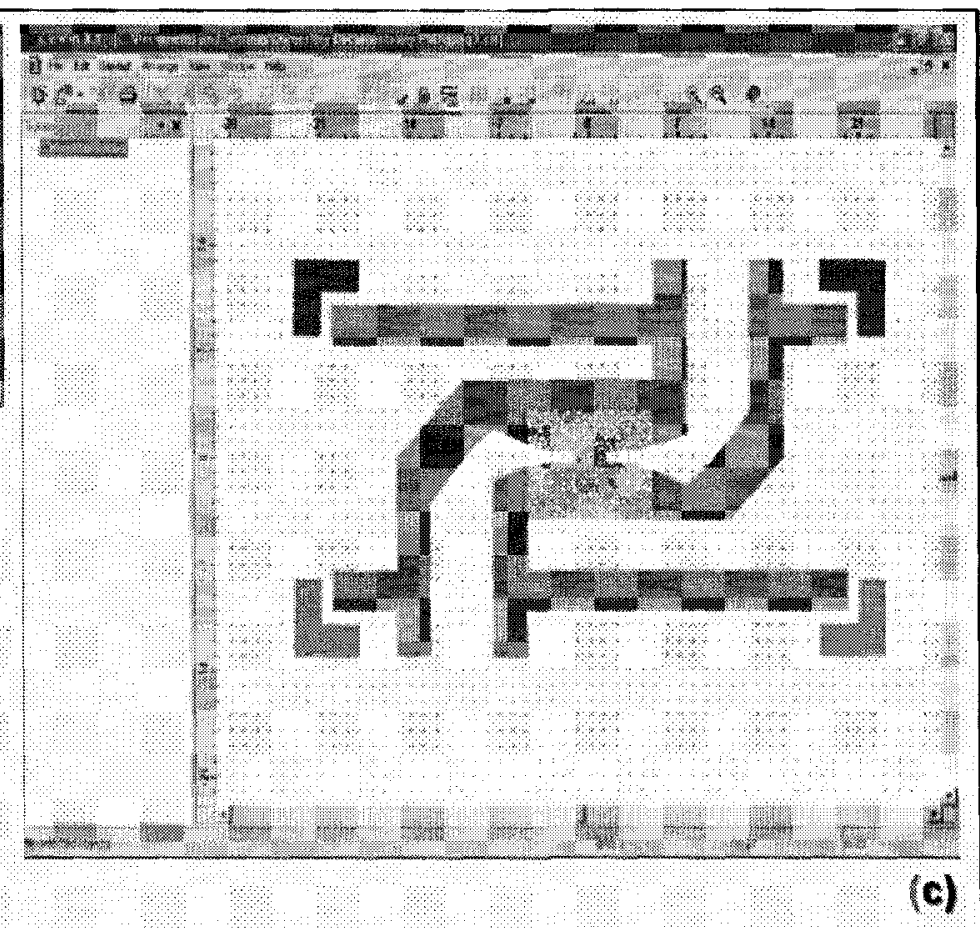

(c)

Figure 4.28. Structures to be built to study CAM states on PhQs. (a) $10 \mu \mathrm{m} \times 10 \mu \mathrm{m}$ PhQ structure, (b) $40 \mu \mathrm{m} \times 20 \mu \mathrm{m}$ PhQ structure, and J-coupler implementation with (c) 10 $\mu \mathrm{m} \times 10 \mu \mathrm{m}$ PhQ structure.

Image (a) is the $10 \mu \mathrm{m} \times 10 \mu \mathrm{m}$ PhQ structure to be written by the EBL system and the align marks shown are to be used with the photolithography waveguide mask. Image (b) is a larger version of the PhQ that has $40 \mu \mathrm{m} \times 20 \mu \mathrm{m}$ dimensions. The third system (c) is a $10 \mu \mathrm{m} \times 10 \mu \mathrm{m} \mathrm{PhQ}$ structure and J-coupler ports. It was initially designed in COMSOL®, but the $\mathrm{PhQ}$ pattern requires different exposure patterns parameters than the J-coupler system. Thus, the PhQ pattern needs to be processed differently than that of the J-couplers.

The PhQ portion of system (c) in the COMSOL® program was cropped and exported to Photoshop® in order to enlarge features required for etching compensation. Images (a) and (b) of figure 4.29 show this process. 


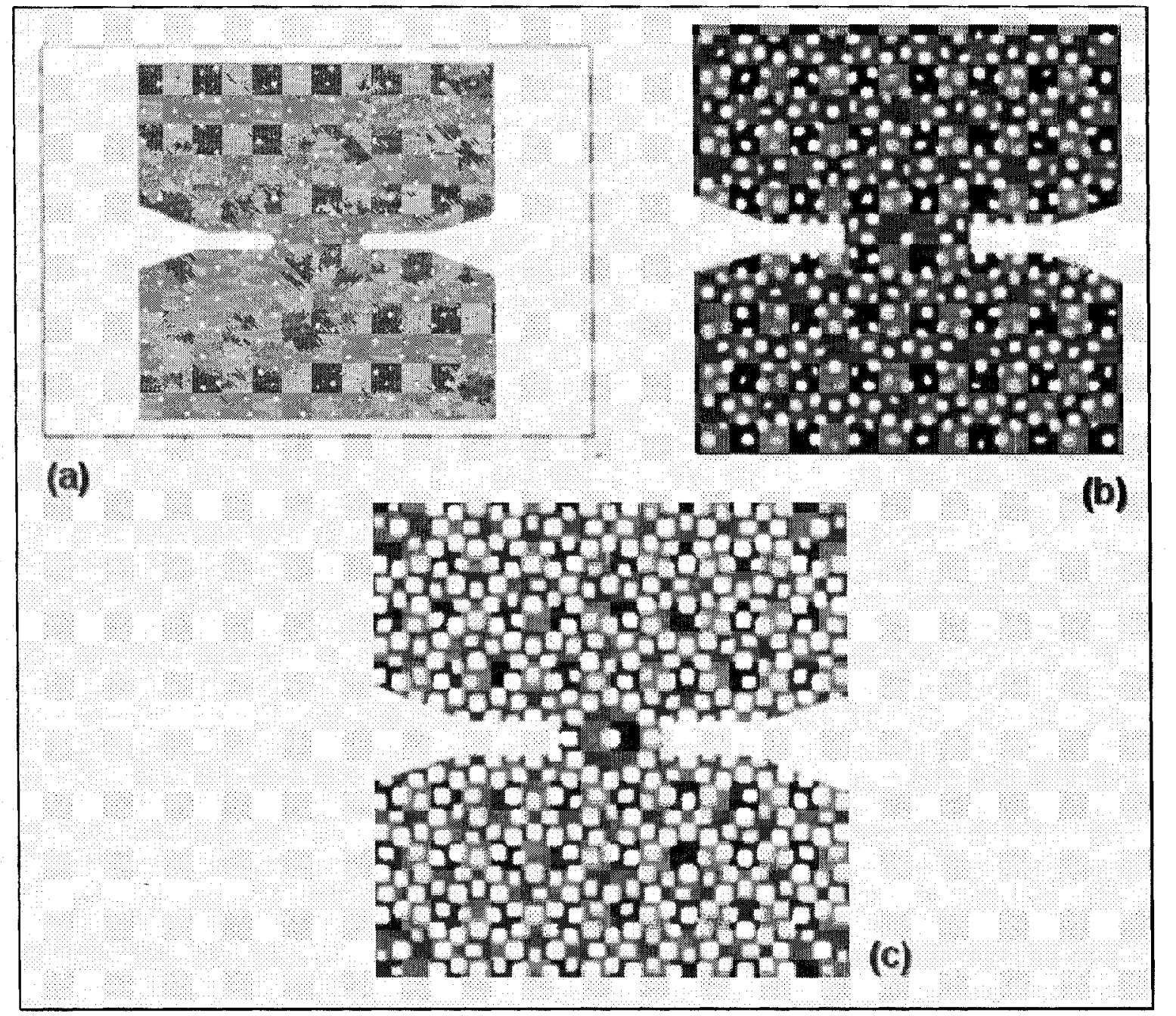

Figure 4.29. Preparation of PhQ pattern for integration with J-coupler system. (a) COMSOL® image of PhQ portion cropped from a J-coupler-PhQ system. (b) Photoshop@ image of cropped PhQ pattern with structure swelled to compensate for development and etching. (c) Larger swelling required for cropped pattern because swelling of (b) was not enough, yielding smaller rods.

Image (c) is a larger swell of the PhQ pattern because the swelling of (b) was not enough to compensate for the development and etching process (i.e. the rods turned out smaller than they should have been). The idea behind this J-coupler-PQ system will be used to help increase CAM state efficiency. From Chapter 3, this PhQ pattern is a possible candidate to physically demonstrate the Lamb shifted CAM states.

Figure 4.30 shows the fabricated PhQ-waveguide structure of image (a) of figure 4.28. Image (a) of figure 4.30 shows the pattern with the waveguide. One will notice 
that there is no taper region between the waveguide and the $\mathrm{PhQ}$ pattern. This is done so that strong scattering can be generated and CAM states can be excited. Image (b) of figure 4.30 shows a closer image of the fabricated PhQ system. There are unintentional fabrication defects around the perimeter of the pattern, but the center region is almost identical to the computer generated image. The center rod diameter is exactly $250 \mathrm{~nm}$.

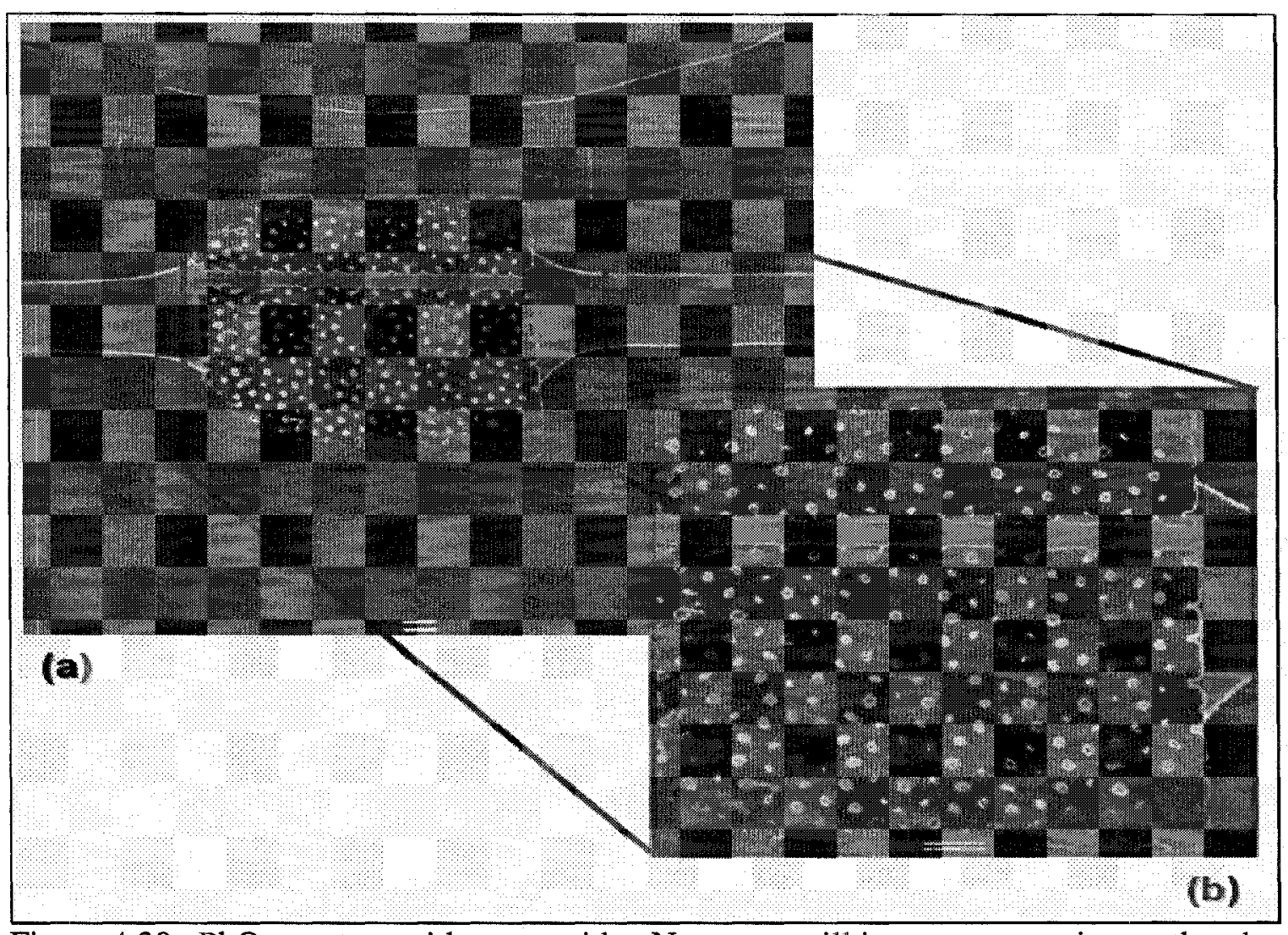

Figure 4.30. PhQ structure with waveguide. No tapers will increase scattering so that the "defect" mode can be seen on the spectrum scan and detected by the IR camera. All bar lengths are one micron.

Figure 4.31 shows the fabricated $40 \mu \mathrm{m} \times 20 \mu \mathrm{m}$ PhQ structure. Image (a) is a larger image showing the waveguide region as well and image (b) is a closer image of the PhQ pattern. The pattern is relatively defect-free, with a few exceptions. This shows that a relatively large intricate pattern can be written by a cost-effective converted SEM system. 


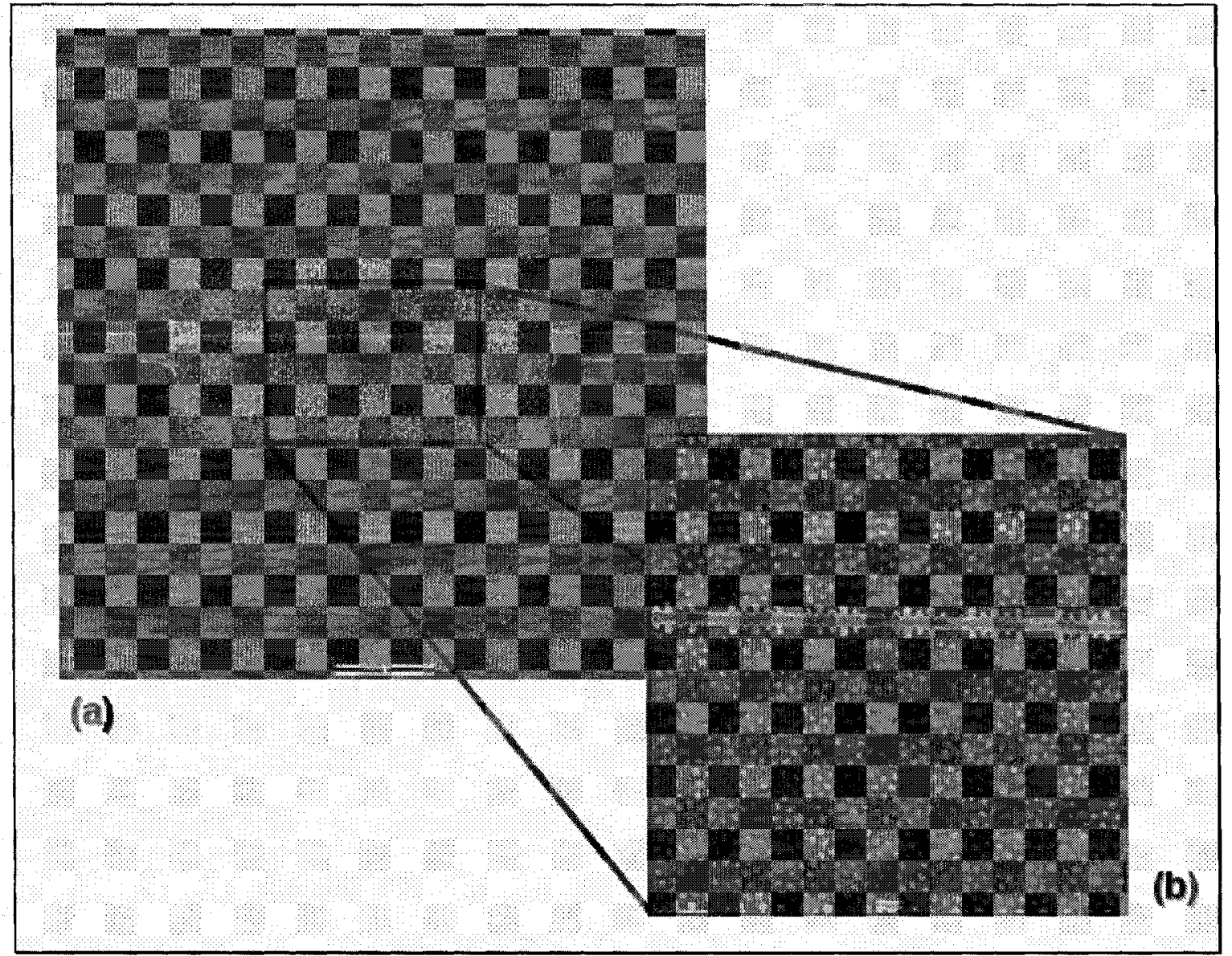

Figure 4.31. Fabricated larger PhQ system. (a) PhQ waveguide system. Bar length is 10 microns. (b) Closer image of PhQ pattern, where bar length is one micron.

Figure 4.32 shows the fabricated J-coupler-PhQ system. Image (a) shows the entire region with the waveguides. The arrows in the image point to mismatches between the EBL J-coupler-PhQ pattern and the photolithography waveguide. 


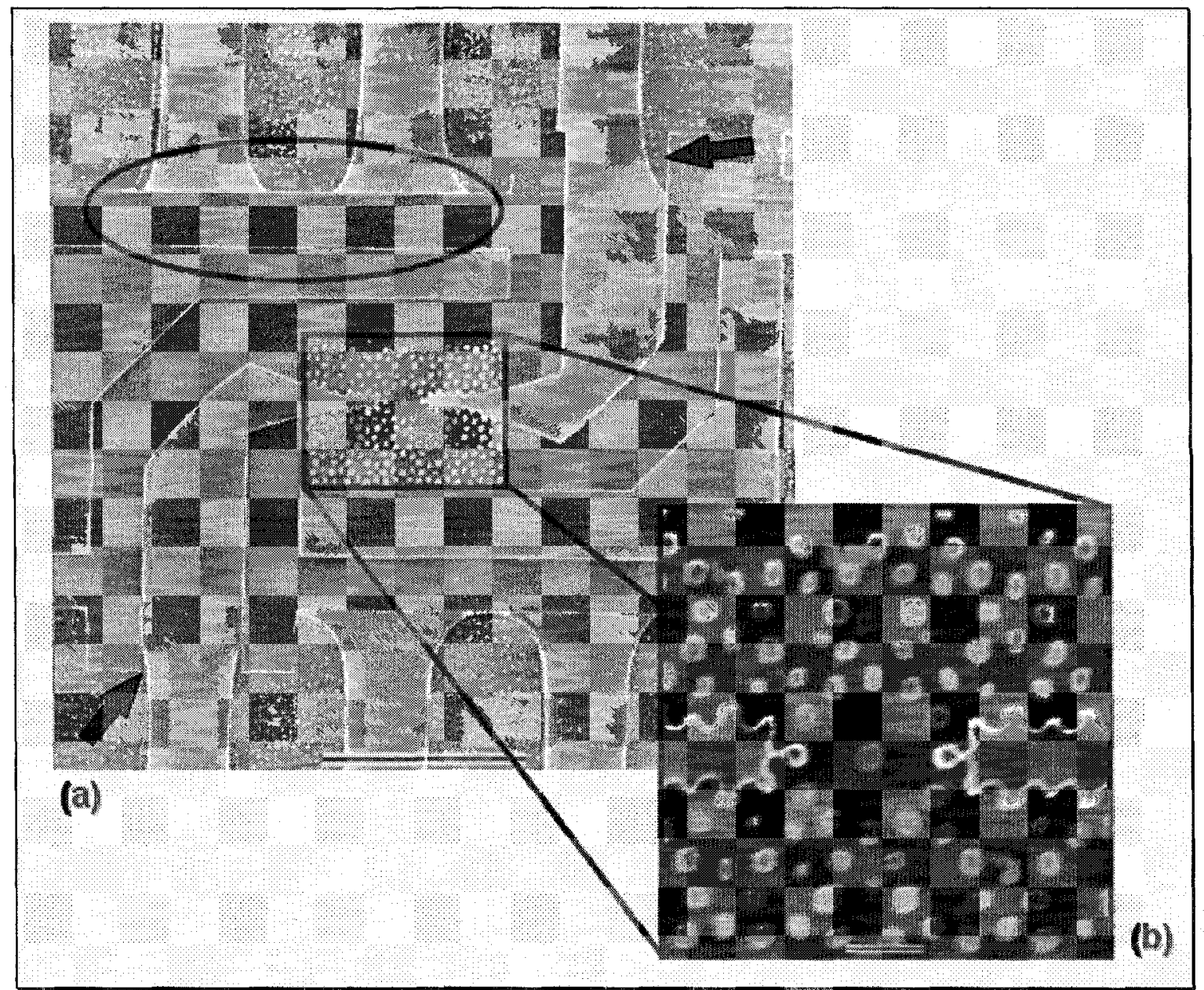

Figure 4.32. J-coupler-PhQ system. (a) Large image showing the entire system. Arrows point to mismatches between EBL pattern and photolithography of waveguides. This will lead to scattering loss. The ellipse shows the EBL regions written in intentionally to keep the other waveguides in the photolithography mask from coming near the PhQ system. This will help reduce waveguide-waveguide coupling which would take energy away from the PhQ system. Bar length is 10 microns. (b) Close up of PhQ pattern. bar length is one micron.

This completes the device fabrication section. The next chapter examines device measurement. 


\section{Measurement and Results}

This chapter will present the measurement process for the structures proposed in Chapter 3 and fabricated in Chapter 4 . The chapter will describe two independent measurement stations, one at the National Research Council (NRC) of Canada, and one at Carleton University, and the results obtained from each facility will also be discussed. The results will show very close agreement with a priori simulated CAM states. In addition, other experimental results will appear, and when simulated a posteriori also agree closely.

\subsection{NRC Measurements}

This section will discuss the NRC setup specifically, however the setup of both NRC and Carleton are very similar. Figure shows a schematic view of the measurement setup. One has a source input from a tunable laser. The light is carried to the input fiber positioner with a tapered fiber. The light is then coupled to the sample where it is mounted on another positioner. The output of the light is then couplered into to a third positioner where a lens or another tapered fiber. If it is a lens system, the output is either

directed to a $\mathrm{CCD}$ camera for imaging or a Ge detector and then on to a computer for analysis. If the output is a tapered fiber, then it is connected to an OSA. The next subscetion will deal with the NRC system exclusively. 


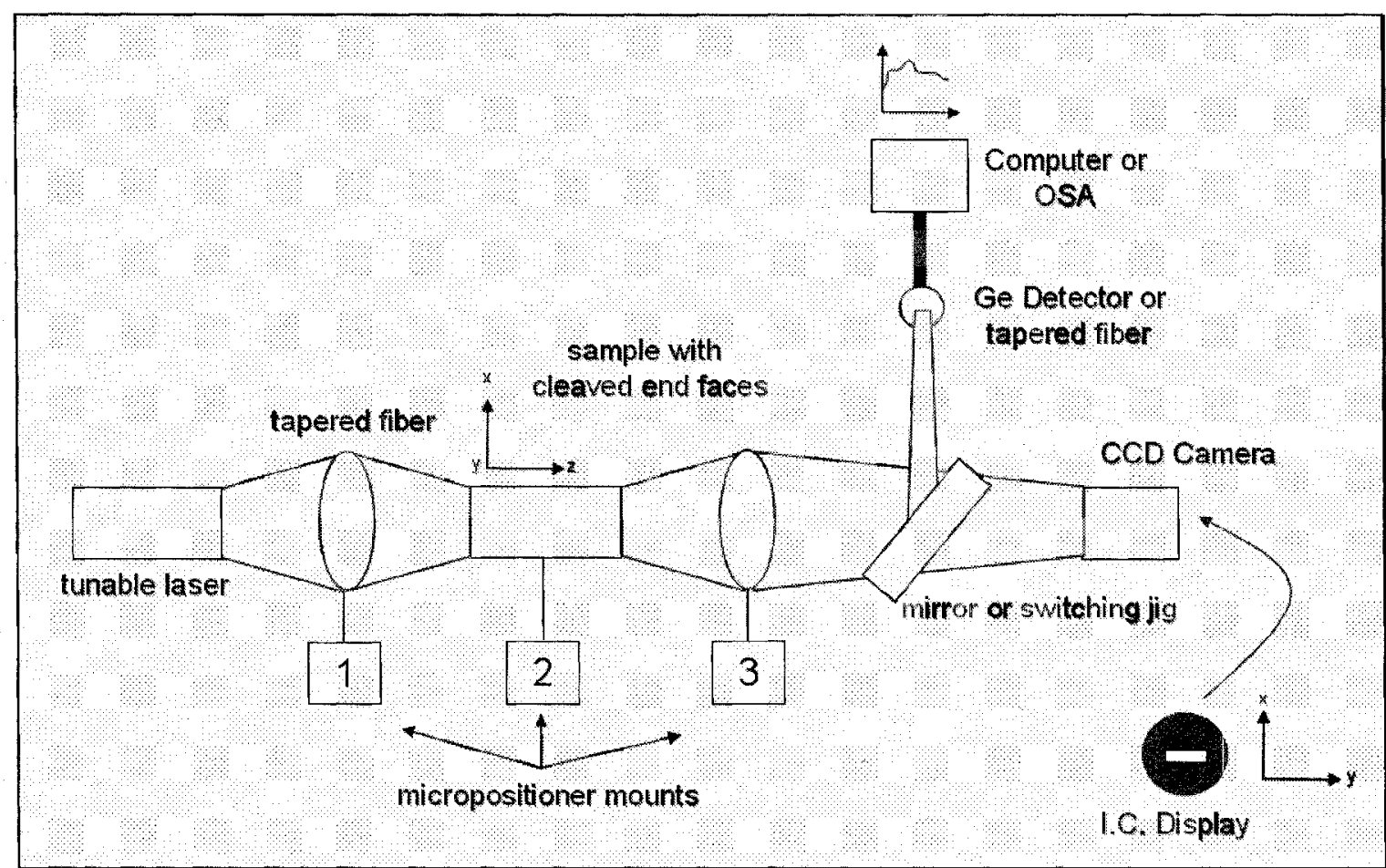

Figure 5.1. General schematic for both systems used. The input stage consists of a tunable laser, and an input positioner 1. The sample sits on the positioner 2 and the output system begins at positioner 3 . The output can then either be directed to a CCD camera and an OSA or computer system that models with output.

\subsubsection{Measurement Setup and Equipment}

Figure 5.2 shows an image of the entire setup and an image of the area where the sample is placed for testing. Arrow (a) in figure 5.2 points to the Agilent ${ }^{\circledR} 81600 \mathrm{~B} 150$ tunable laser source with a wavelength from $1450 \mathrm{~nm}$ to $1590 \mathrm{~nm}$. It has a wavelength resolution of $0.1 \mathrm{pm}$ and a maximum power output of $3 \mathrm{~mW}$ [54]. The output power depends upon the wavelength range. The laser output is first passed through a free space coupler in which an optical polarizer selects TE or TM modes. The output of the free space coupler is connected to a tapered fiber. The taper has a $2 \mu \mathrm{m}$ core size with a $15 \mu \mathrm{m}$ convergence length. The tapered fiber is then placed in a fiber leaf holder, shown by arrow (b). Arrow (c) points to a custom made sample holder for holding small structures. Through the overhead microscope, the fiber tip is aligned with the sample. 


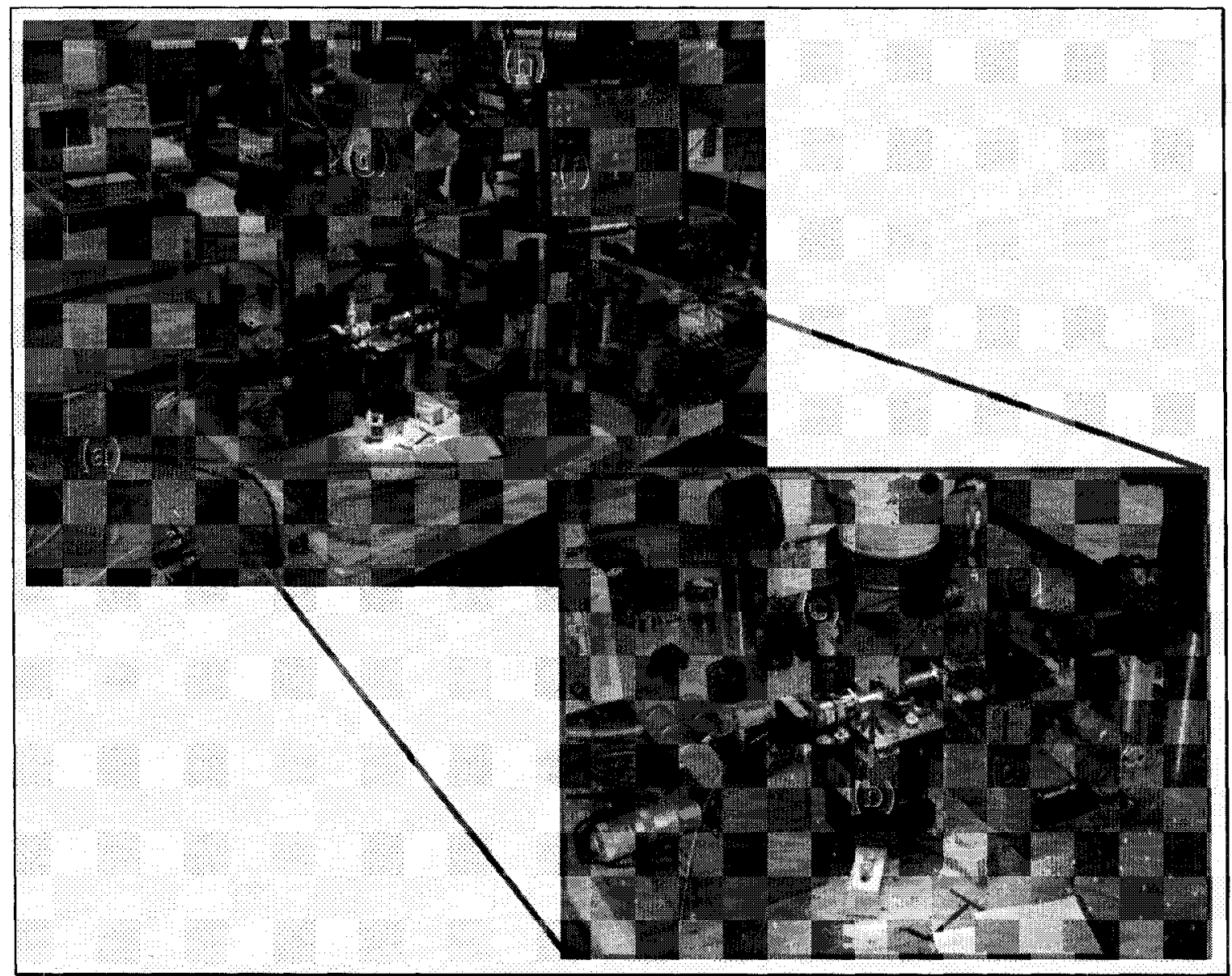

Figure 5.2. Measurement Setup at NRC. (a) Laser source, (b) tapered fiber input, (c) sample holder, (d) 40x objective output lens, (e) output filters, (f) optical detector, (g) IR output camera, (h) computer running LabVeiwß system. [Courtesy of Chris Raum]

The output of the sample is imaged to either a CCD camera (g), or a photodector (f), via a 40x objective (d). The output of the lens passed through another polarizer (e). The photodetector is connected to a computer $(\mathrm{h})$ running LabView $(B)$ software. This software controls wavelength scans for the optical measurements of devices under test.

\subsubsection{Measurement Process}

The actual measurement process requires a few steps. First, the sample is carefully placed on the sample holder. The microscope is then used to align the tapered fiber to the input of the waveguide that leads to the PhQ region. 'The mirror that redirects output light is placed so that light is redirected into the IR camera. The NRC setup has been 
carefully calibrated to immediately obtain fringes at the output of the objective. The sample is then slowly raised to a vertical position until the fringes have large separation. This occurs until the fringes completely disappear and a bright spot occurs. This spot is the waveguide output. Figure 5.3 shows the outputs of two waveguides, (a) is a $3.5 \mu \mathrm{m}$ waveguide and (b) $5 \mu \mathrm{m}$ waveguide.

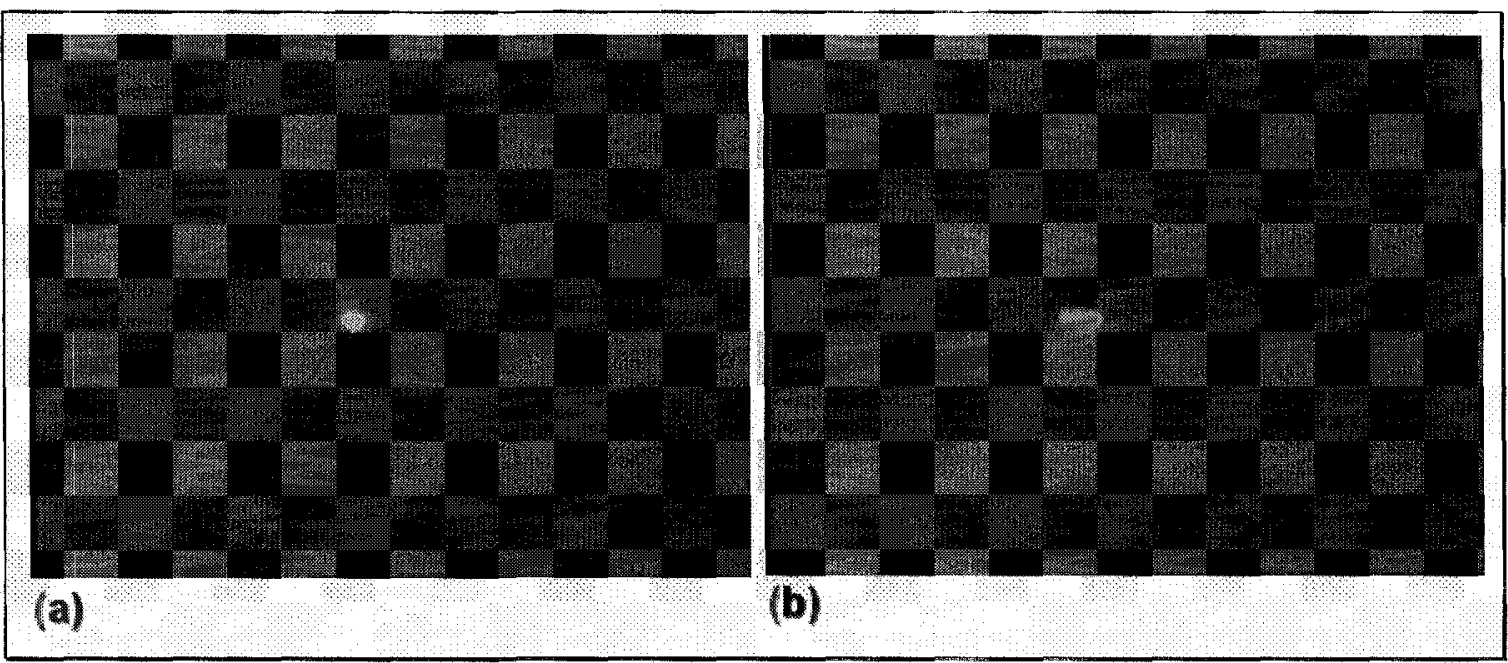

Figure 5.3. Waveguide outputs. (a) Optical output of $3.5 \mu \mathrm{m}$ width straight test waveguide and (b) $5 \mu \mathrm{m}$ width straight test waveguide.

These are SOI waveguides with a top silicon thickness of $250 \mathrm{~nm}$ and an oxide thickness of $1 \mu \mathrm{m}$. The height-width combination is not a single-mode condition but image (a) of the $3.5 \mu \mathrm{m}$ waveguide appears single mode, while the $5 \mu \mathrm{m}$ waveguide shows a higher order mode profile.

Once the output has been detected with the IR camera, the mirror is then used to redirect the output into the photodetector. The photodetector measures the output power. The sample stage controls are adjusted for optimal output power. Once this has been completed, the LabView® software can be set to control the tunable laser output scan range and rate. Then a scan can be initiated. LabView(B) software reads in the photodetector output and displays the results. 


\subsubsection{Measurement Result: System One}

The first system to be tested was the structure built and shown in figure 4.30 . Figure 5.4 shows the results of two scans $(0.1 \mathrm{~nm}$ resolution): (a) a $3.5 \mu \mathrm{m}$ width straight waveguide scan and (b) the waveguide containing PhQ structure.

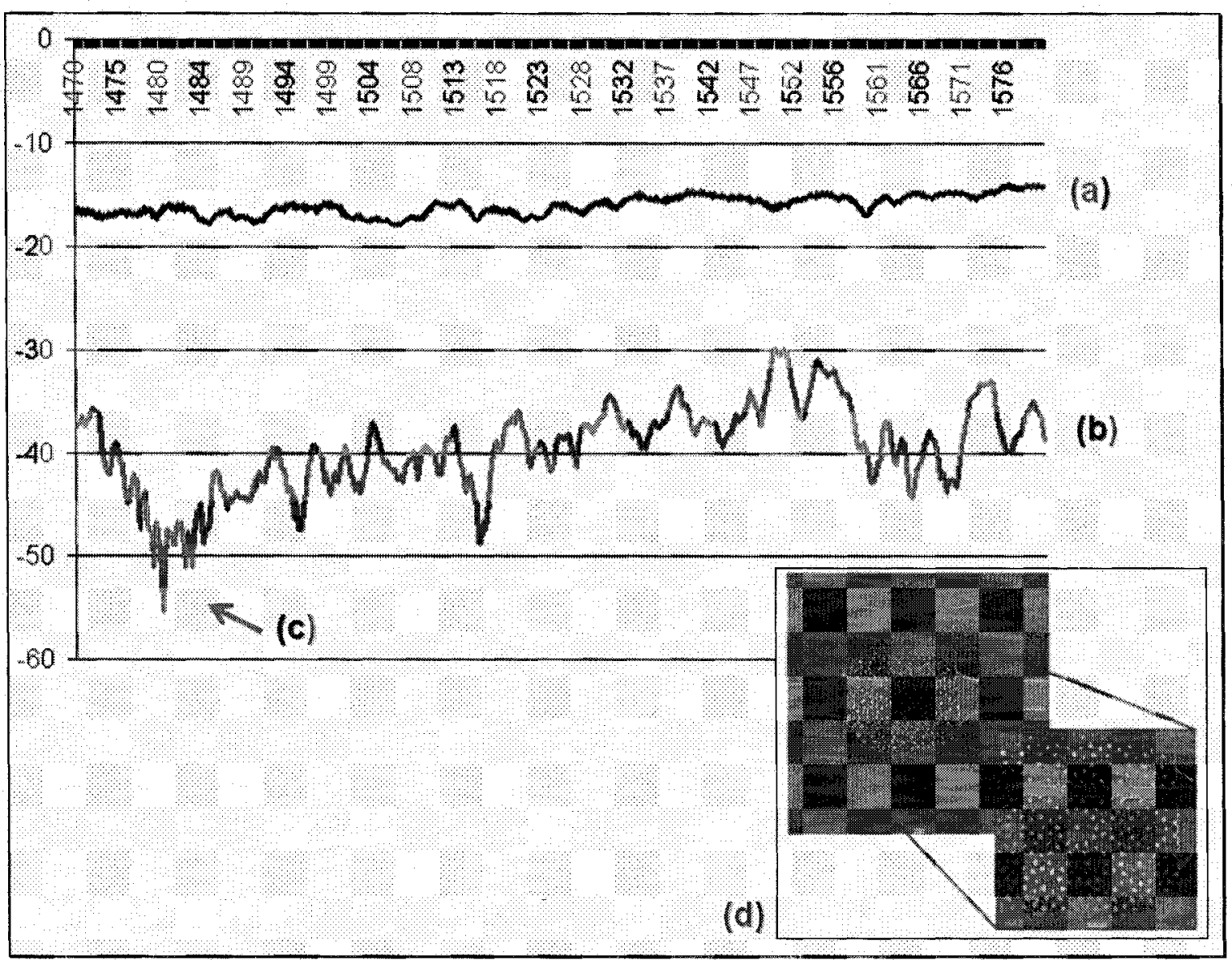

Figure 5.4. Scan of straight waveguide vs. scan of waveguide containing PhQ (i.e. figure 4.30). (a) Straight waveguide scan. (b) Waveguide containing PhQ scan. (c) Large dip near simulated wavelength of defect mode wavelength of $1484 \mathrm{~nm}$. (d) Picture of the fabricated PhQ structure being tested.

Curve (a) shows the output scan of the straight waveguide. Curve (b) accordingly has more loss because of the no taper region, however the noticeable dips are due to the CAM states of the PhQ pattern. Arrow (c) points to a relatively large dip that is near the wavelength $1480 \mathrm{~nm}$ in which there was $m=1$ found in image (b) of figure 3.51, and redrawn again in figure 5.5. Since the simulation was of the electric field, it would make 
sense to simulate the power as well, since intensity is what is detected. Figure 5.6 is a time-harmonic simulation of (a) electric field and (b) power at $1484 \mathrm{~nm}$. The simulated structure is shown in (c).

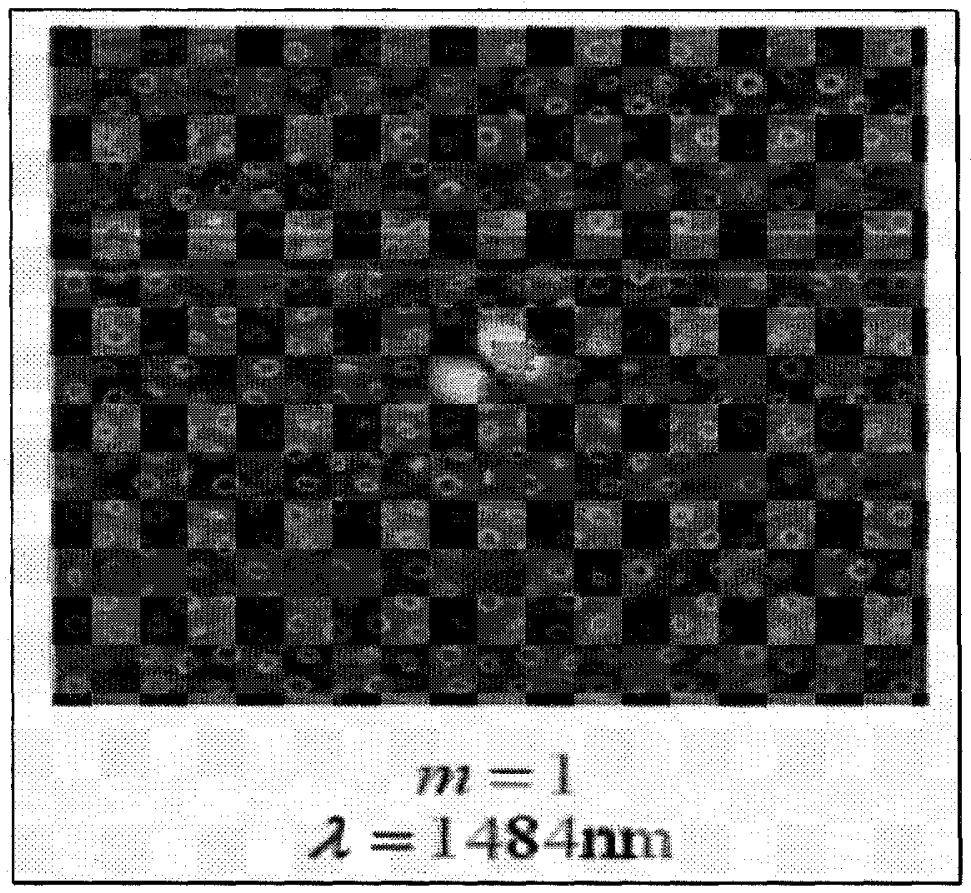

Figure 5.5. Simulated $m=1 \quad$ CAM state with waveguide. 


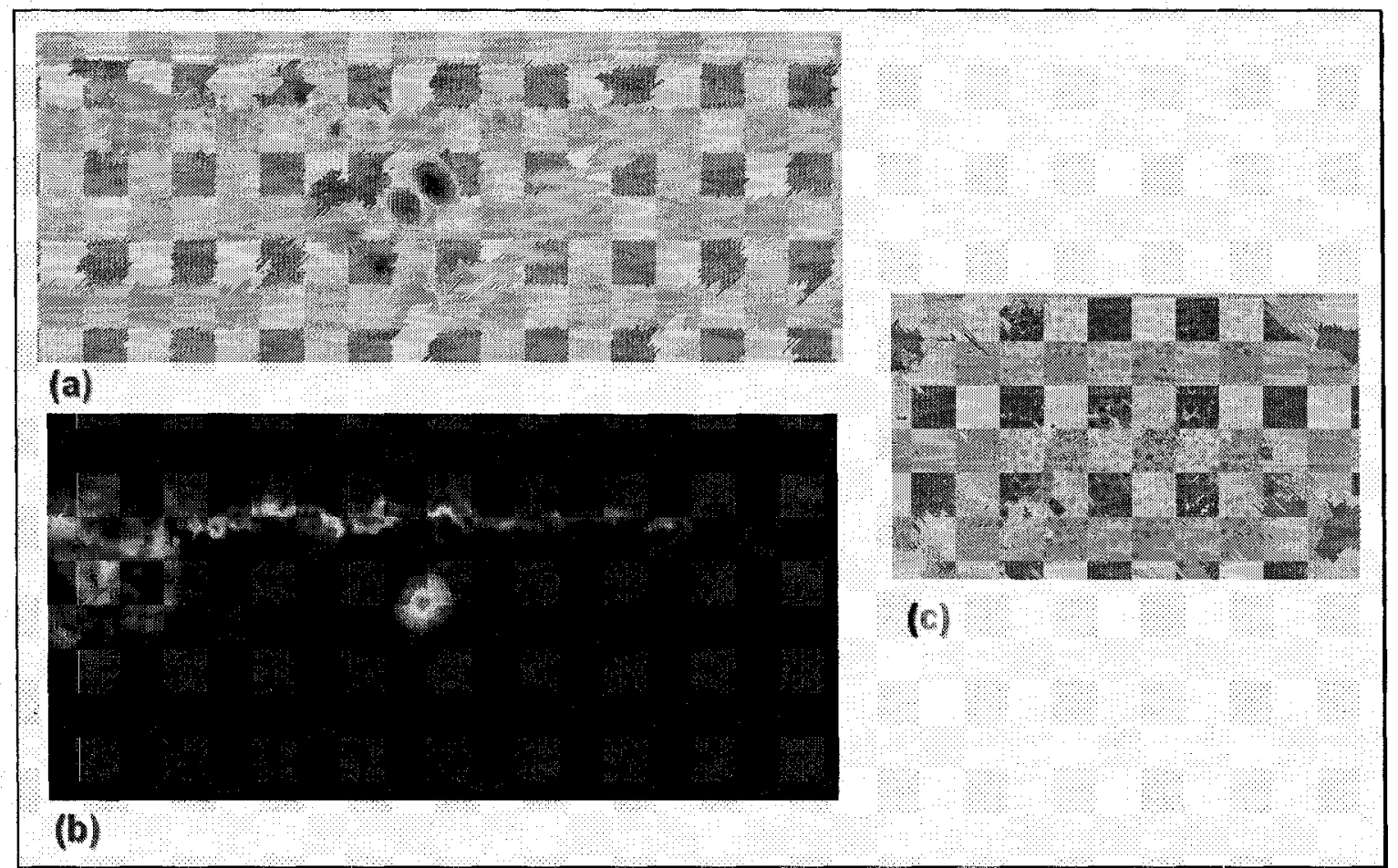

Figure 5.6. Time-harmonic simulations of dip wavelength $1484 \mathrm{~nm}$. (a) the electric field, (b) the power and (c) the simulated structure.

It shows a strong $m=1$ CAM state at the center of the PhQ pattern. This comparison between experimental results and simulation is very motivating. The difference between the simulated wavelength and the experimental wavelength is most likely due to unintended fabrication effects. However, the difference is small enough to conclude that the simulation and experiment agree quite well in relation to the dip and state.

In addition, there were two prominent dips at $1495 \mathrm{~nm}$ and $1517 \mathrm{~nm}$ in figure 5.4. Power simulations at these wavelengths are shown in figure 5.7. They show that at 1495 $\mathrm{nm}$ the power is considerably weaker and in a ring shape at the center of the pattern, while at $1517 \mathrm{~nm}$ the power is stronger than $1494 \mathrm{~nm}$ but weaker than $1484 \mathrm{~nm}$. 


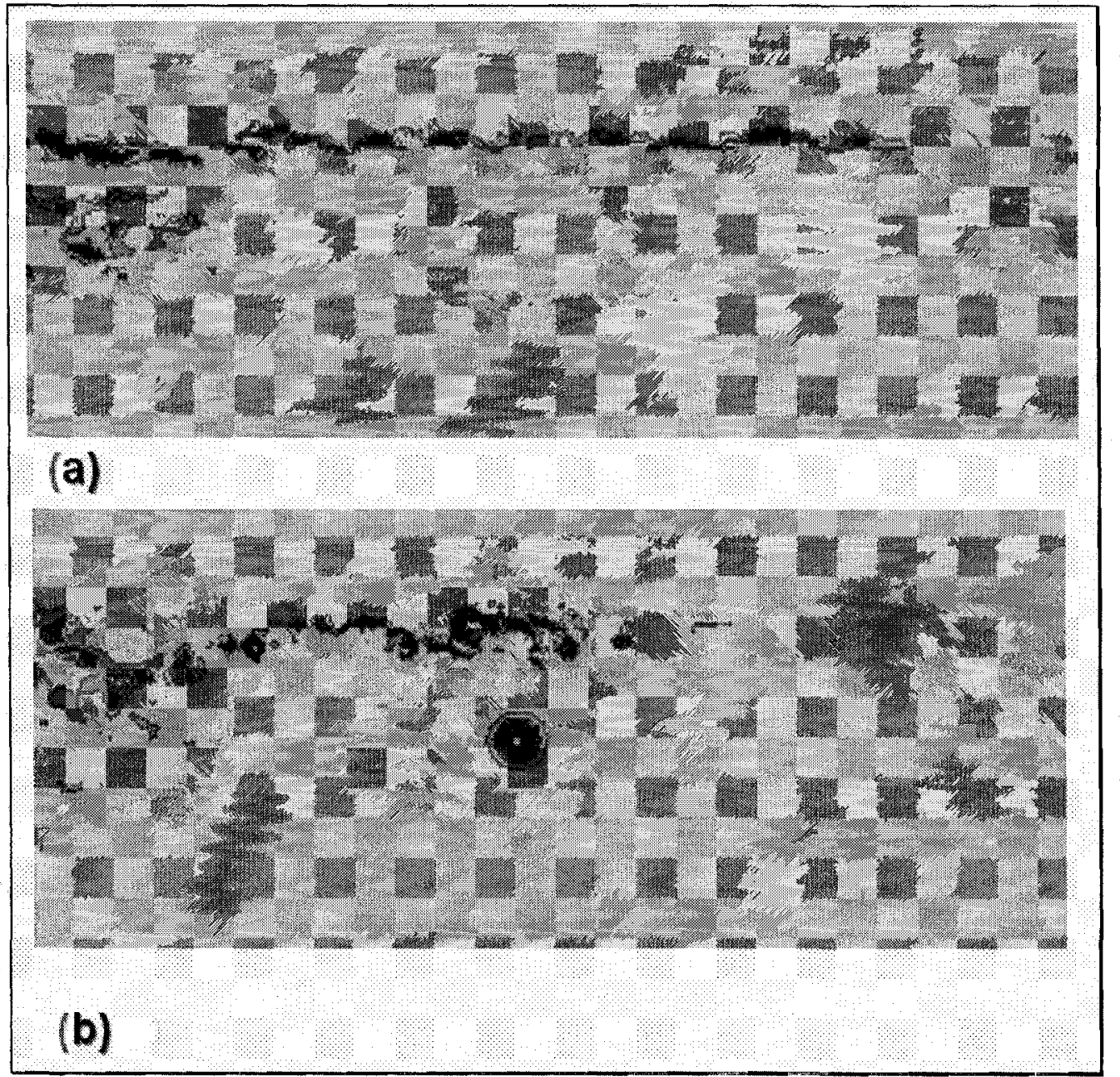

Figure 5.7. Power simulation results of dips at (a) $1495 \mathrm{~nm}$ and (b) $1517 \mathrm{~nm}$.

Two higher resolution $(0.01 \mathrm{~nm}$ resolution) scans were taken of the structure to confirm the presence of the dip. Figure 5.8 shows these two scans (a) and (b). They both showed that the dips were still there. 


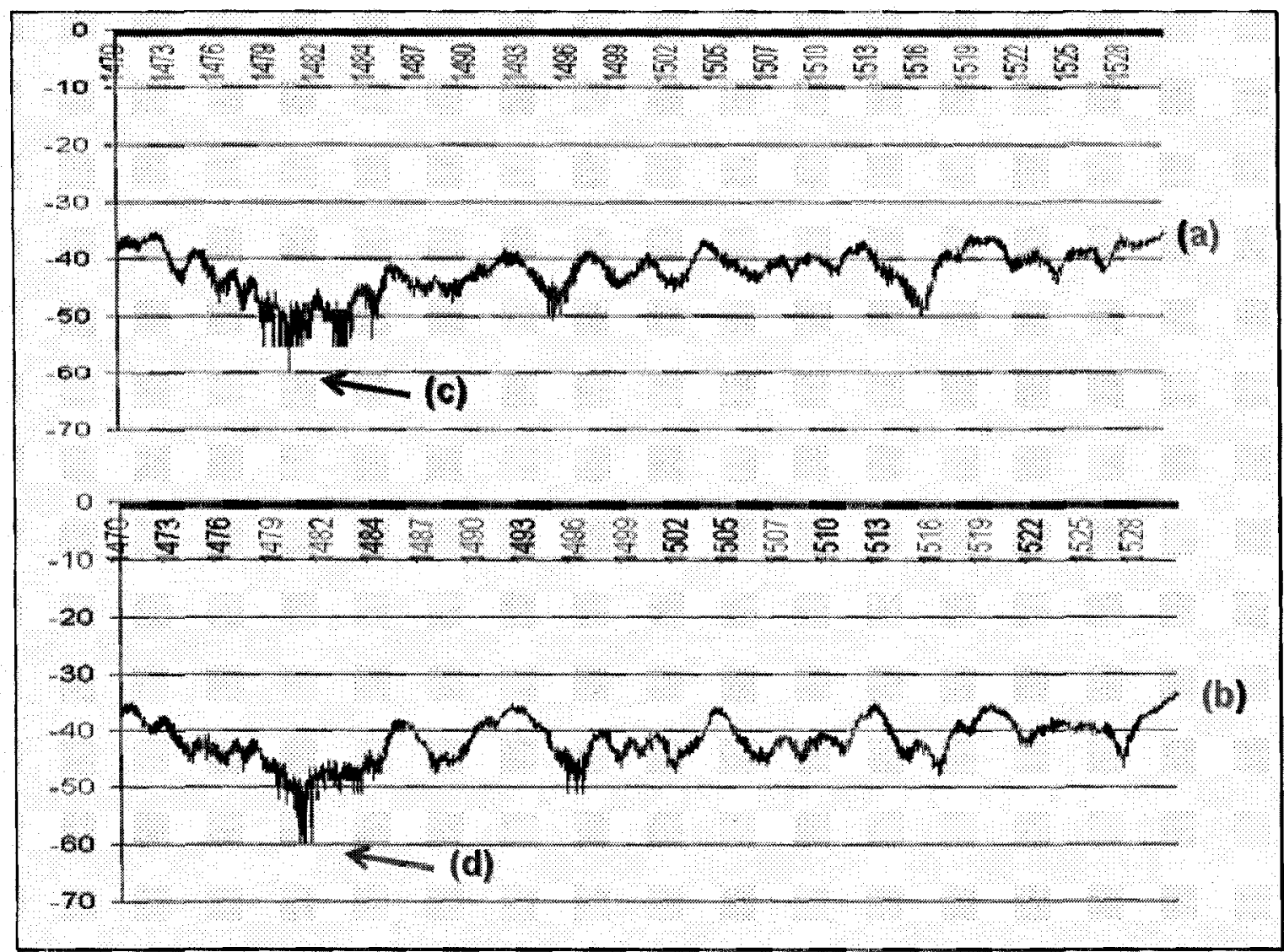

Figure 5.8. Multiple measurements of localized mode wavelength. (a) Wavelength scan with input fiber changed slightly with respect to figure 5.4 measurement. (b) Wavelength scan with input fiber changed slightly with respect to (a) measurement. (c) Dip region at $1484 \mathrm{~nm}$ still prominent while at (d) coupling was reduced.

At the time of these measurements, an overhead imaging station with a very highresolution IR camera and magnification lens was available. This imagining station was used to obtain overhead images of the device while it was being measured. Figure 5.9 shows the results. Picture (a) is the structure. Picture (b), (c) and (d) are the images taken at $1458 \mathrm{~nm}, 1533 \mathrm{~nm}$ and $1484 \mathrm{~nm}$. The images are in false color to show intensity. Images (b) and (c) are non-dip regions that show no clear localization, while image (d) shows a strong localization in the region where the center of the PhQ is located. Image (d) was taken at the exact wavelength that showed localized mode in the center of the PhQ in the simulation. 


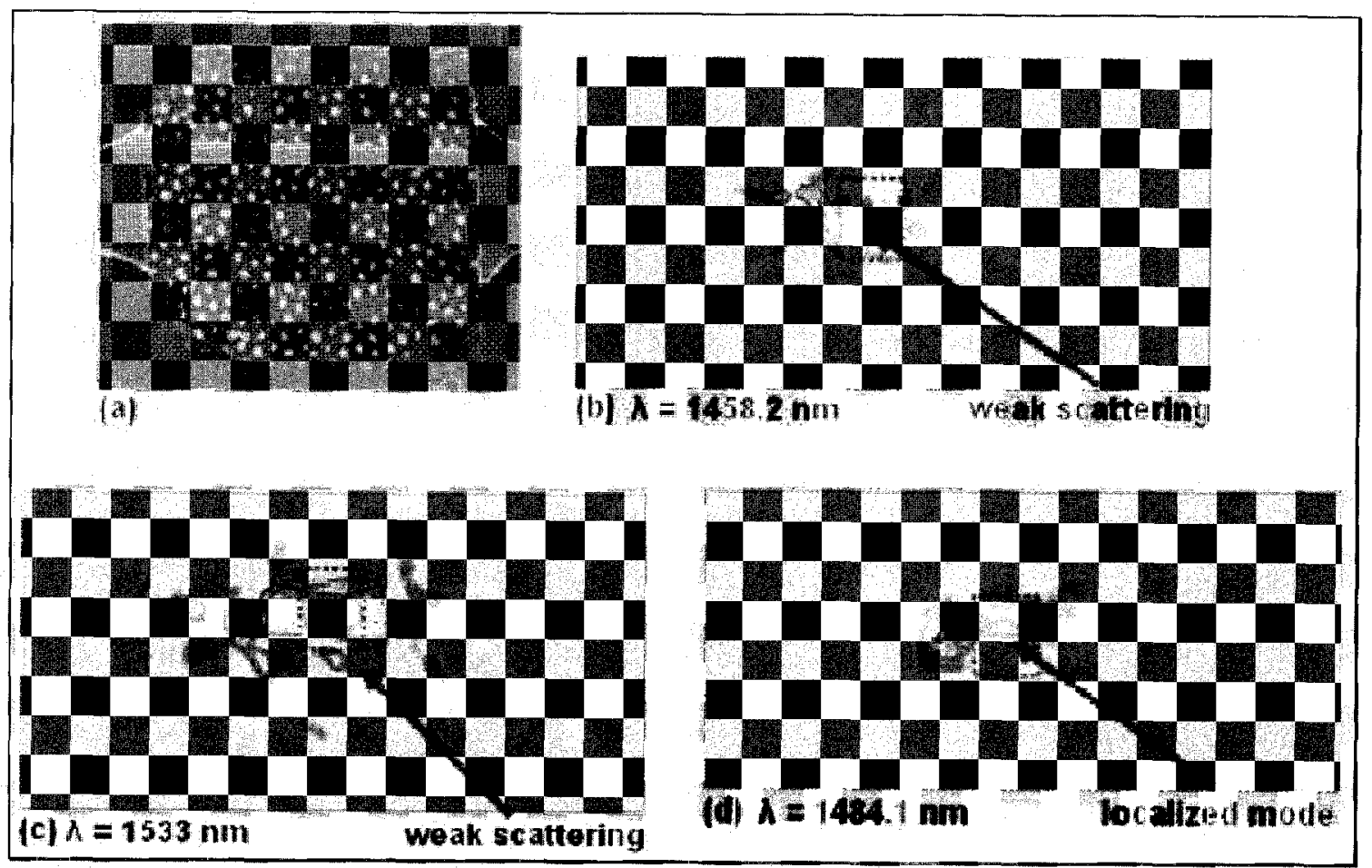

Figure 5.9. Array of IR camera overhead pictures of PhQ. (a) Physical image. (b) and (c) Intensity pictures of the scattering at off-dip wavelength values. Arrows point to weak scattering in the area of the center. (d) Intensity picture of the localized mode shown exactly where the dip region occurs. The dash box represents the outline of the PhQ pattern.

This is the first time that mode localization in PhQs has been observed in this wavelength regime. The agreement between simulation and experiment give strong support to the theory of crystal angular momentum because it verifies that the simulation results are physically accurate.

An a posteriori eigenvalue simulation of the three dips in the far right region of curve (b) of figure 5.4 is shown in figure 5.10. Image (c), (d) and (e) show localized mode profiles around non-center regions. As the next system to measure will show, offcenter regions also have localized modes. These findings, together with the theory of crystal angular momentum, indicate that the bandstructure of a PhQ pattern may be generated in a tight-binding manner, where each site represents an atomic-like system. See Chapter 6 for further discussion on this. 


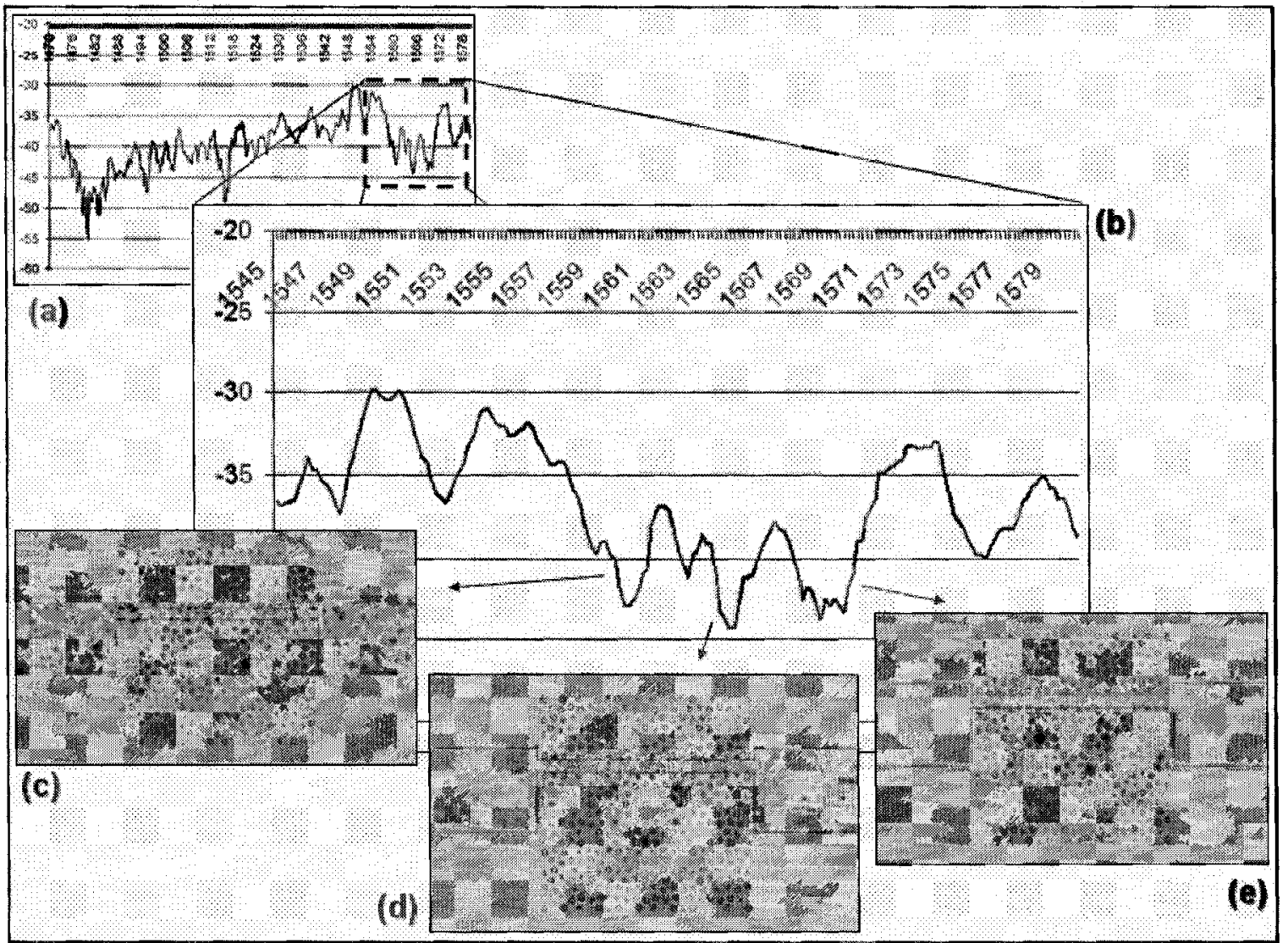

Figure 5.10. Close up on other dip regions. (a) Figure 5.4. (b)Close up of other dip regions. (c), (d), and (e) Eigenvalue simulations of dip wavelengths. 


\subsubsection{Measurement Result: System Two}

The results of system two will show agreement with the previous measurement, further giving evidence to the existence of CAM states.

The next system to measure is the $40 \mu \mathrm{m}$ by $20 \mu \mathrm{m}$ PhQ shown in figure 4.31 .

Figure 5.11 shows the result of three scans. Curve (a), (b) and (c) all have dips in the same region which show consistent behavior for the structure.
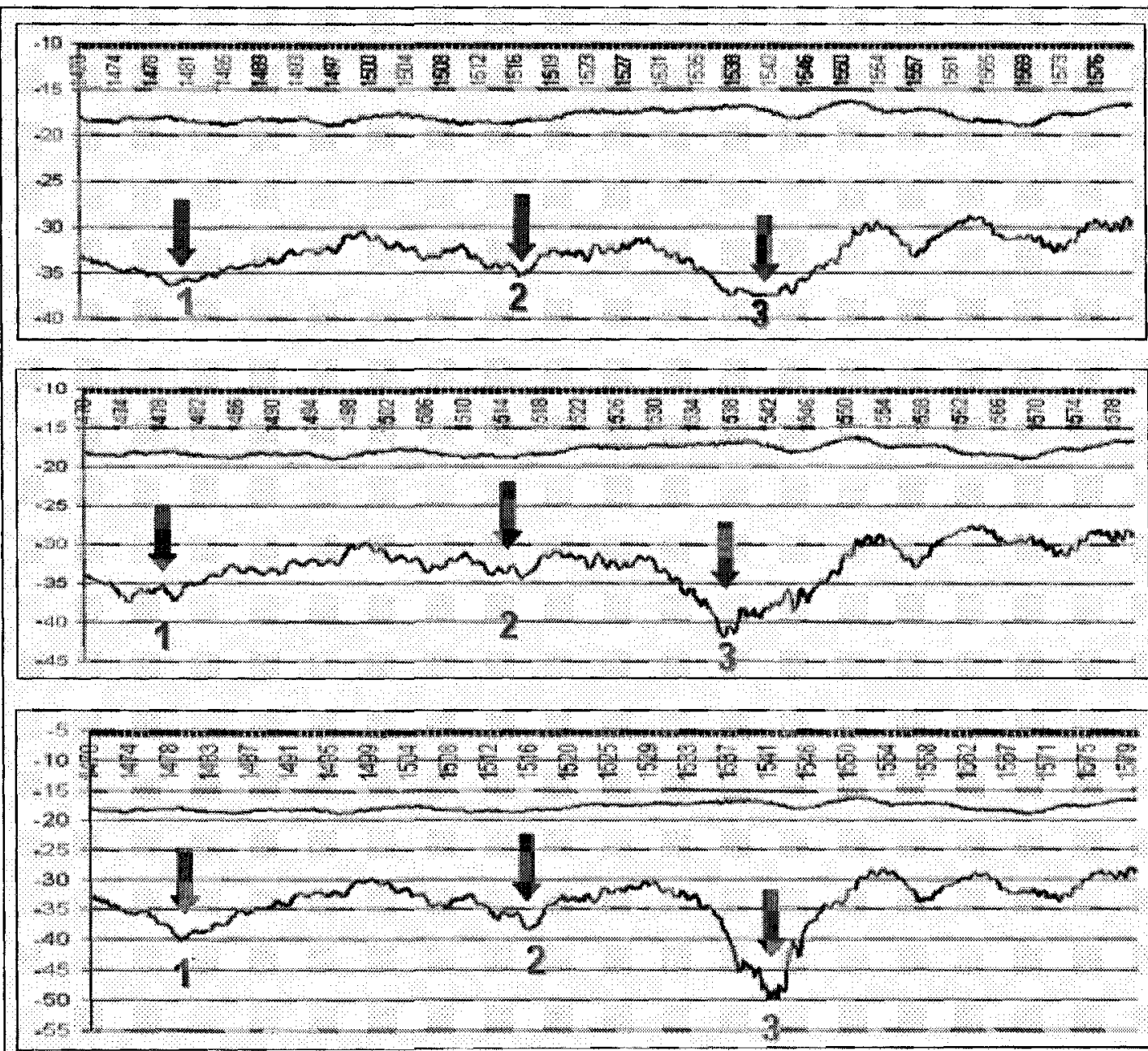

Figure 5.11. Three scans of larger PhQ system. Curve (a), (b) and (c) all have consistent dip regions shown by 1,2 , and 3 . All curves show different coupling strengths. 
Dips 1 and 2 in curves (a) to (c) coincide with two dips at the same wavelengths in the response of the first system. These dips must correspond to the mode profiles of images (b) of both figure 5.6 and figure 5.7, at $1484 \mathrm{~nm}$ and $1517 \mathrm{~nm}$, respectively.

Figure 5.12 shows both system responses together in the same graph.

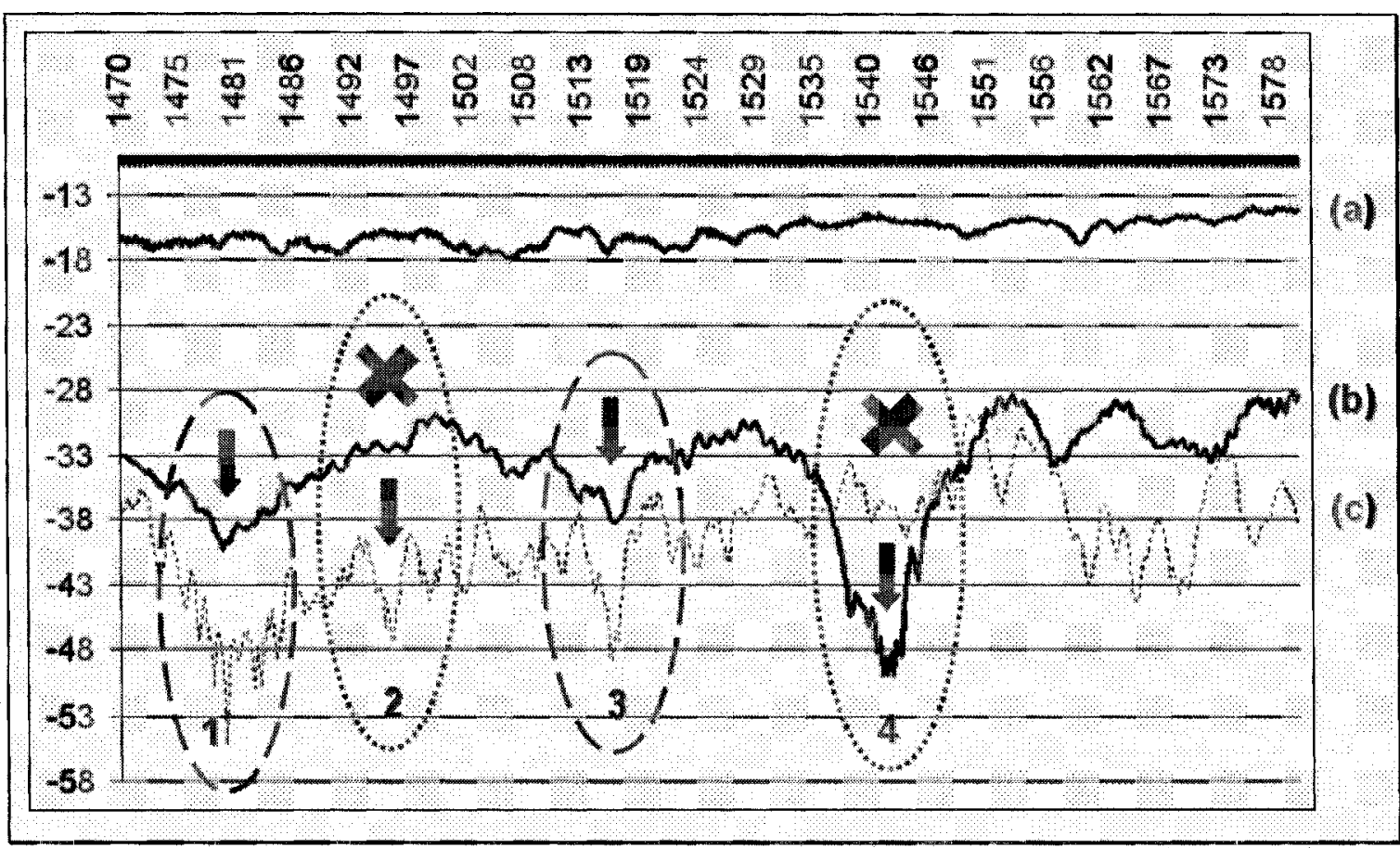

Figure 5.12. System One and Two responses. (a) Straight waveguide response, (b) System 2 response and (c) System 1 response. Dips 1 and 3 coincides with both systems, confirming the existence of the CAM states at the center of the PhQ pattern. Dip 2 is in system 1 but not in system 2 . This is due to the weakness of this state. Dip 4 is in system 2 but not in system 1 because this is associated with a strong, off-center site that is not in system 1. The X symbol indicates a dip on one curve and not in the other.

Curve (a) is the straight waveguide response, curve (b) is the system 2 response, (curve (c) in figure 5.11) and (c) is the system 1 response. Dips 1 and 3 are in excellent agreement when it comes to location. They correspond with the strong mode profiles of the system 1. The X symbol indicates dips in one curve but not in the other. Dip 2 is present in system 1 but not in system 2 . The reason is that the mode associated with this wavelength is already very weak in system 1 , as figure 5.7 (a) indicates. Since system 2 is a lot bigger, and this site is farther away from the input scattering source, it will not be 
present in the scan of system 2. Dip 4 is present in system 2 , but not in system 1 because it is associated with a strong off-center site that is not present in system 1. Figure 5.11 shows this dip is consistently there, however, with different strength. From simulations, there are three off-center eigenmodes with wavelengths very close in range. The eigenmode profiles are shown in figure 5.13. They appear to be $m=0$ like modes.

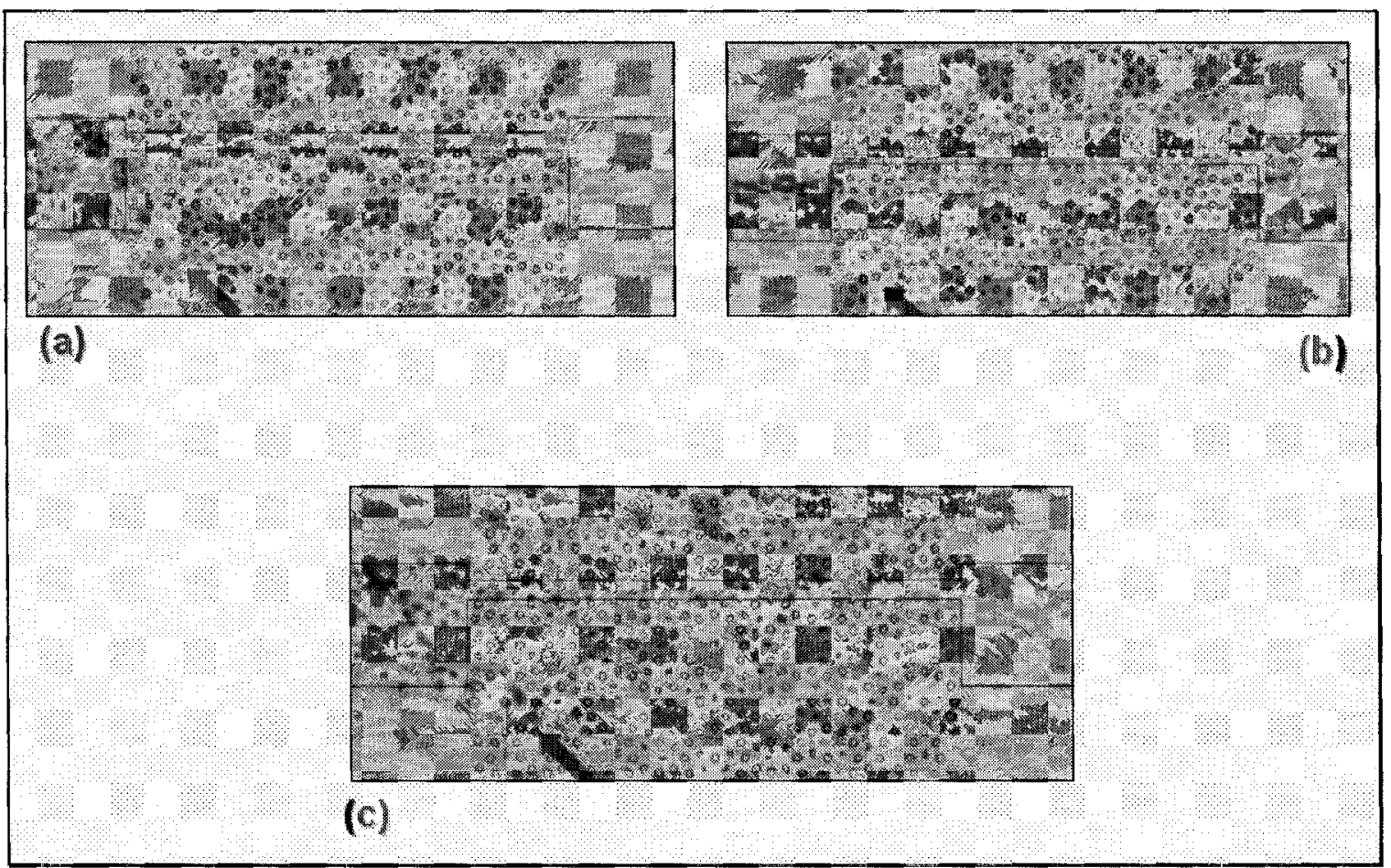

Figure 5.13. Three different strength eigenmode profiles of dip 3 wavelength of figure 5.11. Each eigenmode has slightly different eigenvalues, implying they are degenerate modes whose degeneracy is lifted by pattern structure. There/their/the strength would also depend upon coupling angles, etc.

Because they are close in range, this implies the three modes belong to a triply degenerate mode whose degeneracy is lifted via the pattern.

Figure 5.14 shows the simulated eigenmodes and their associated power simulation at $1484 \mathrm{~nm}$ and $1517 \mathrm{~nm}$. These are the same eigenmodes found in the first system. The measurement results of the two PhQs provide further evidence to the existence of these CAM states. 


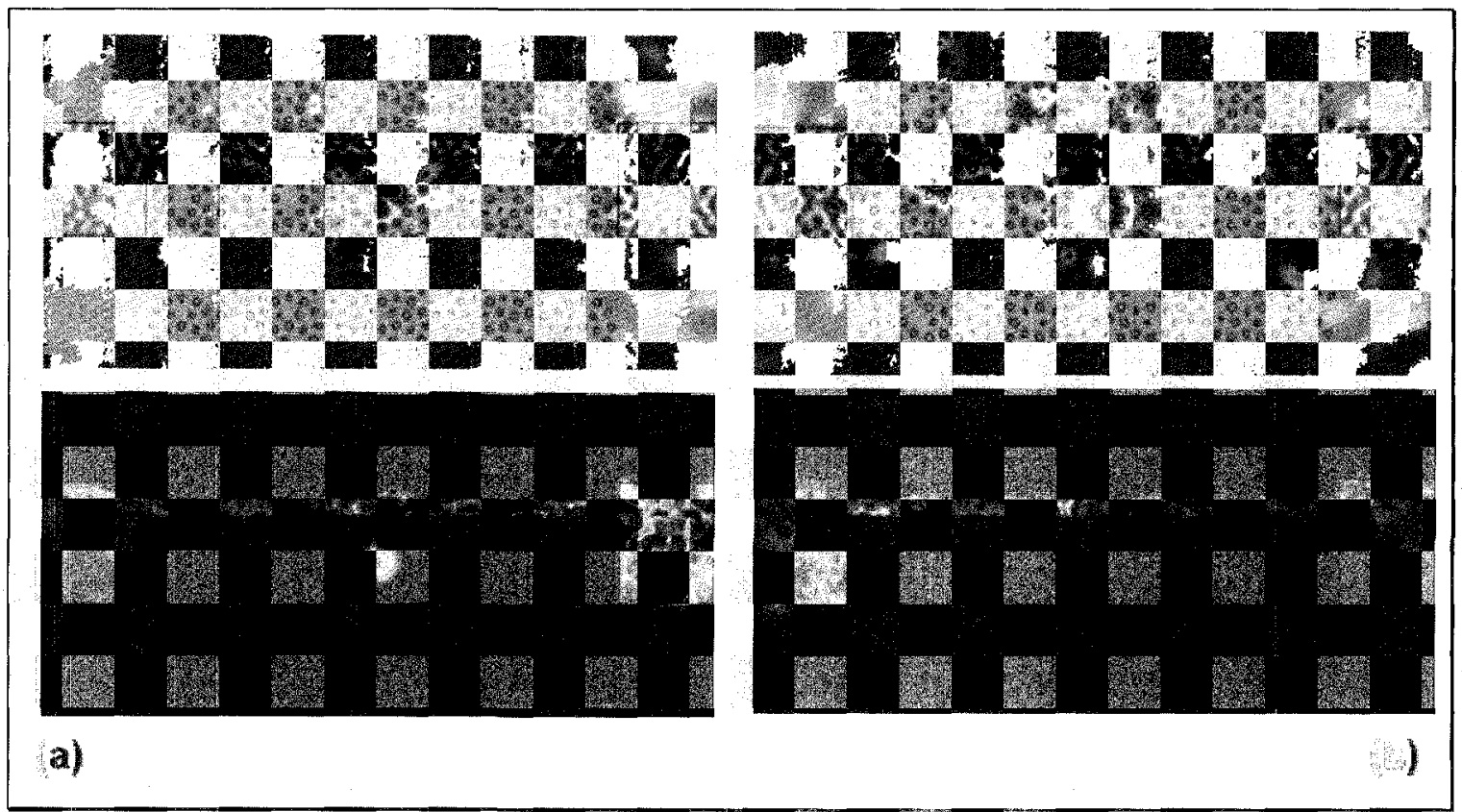

Figure 5.14. Simulated eigenmodes at (a) $1484 \mathrm{~nm}$ and (b) $1517 \mathrm{~nm}$ in the larger system. 


\subsection{Carleton University Measurements}

\subsubsection{Measurement Setup and Equipment}

Self-sufficiency is key to rapid turn around. As such, a testing facility was designed at Carleton University for testing PhQ devices. It was originally built to perform laser trapping experiments. Figure 5.15 shows the basic setup required to perform the testing.

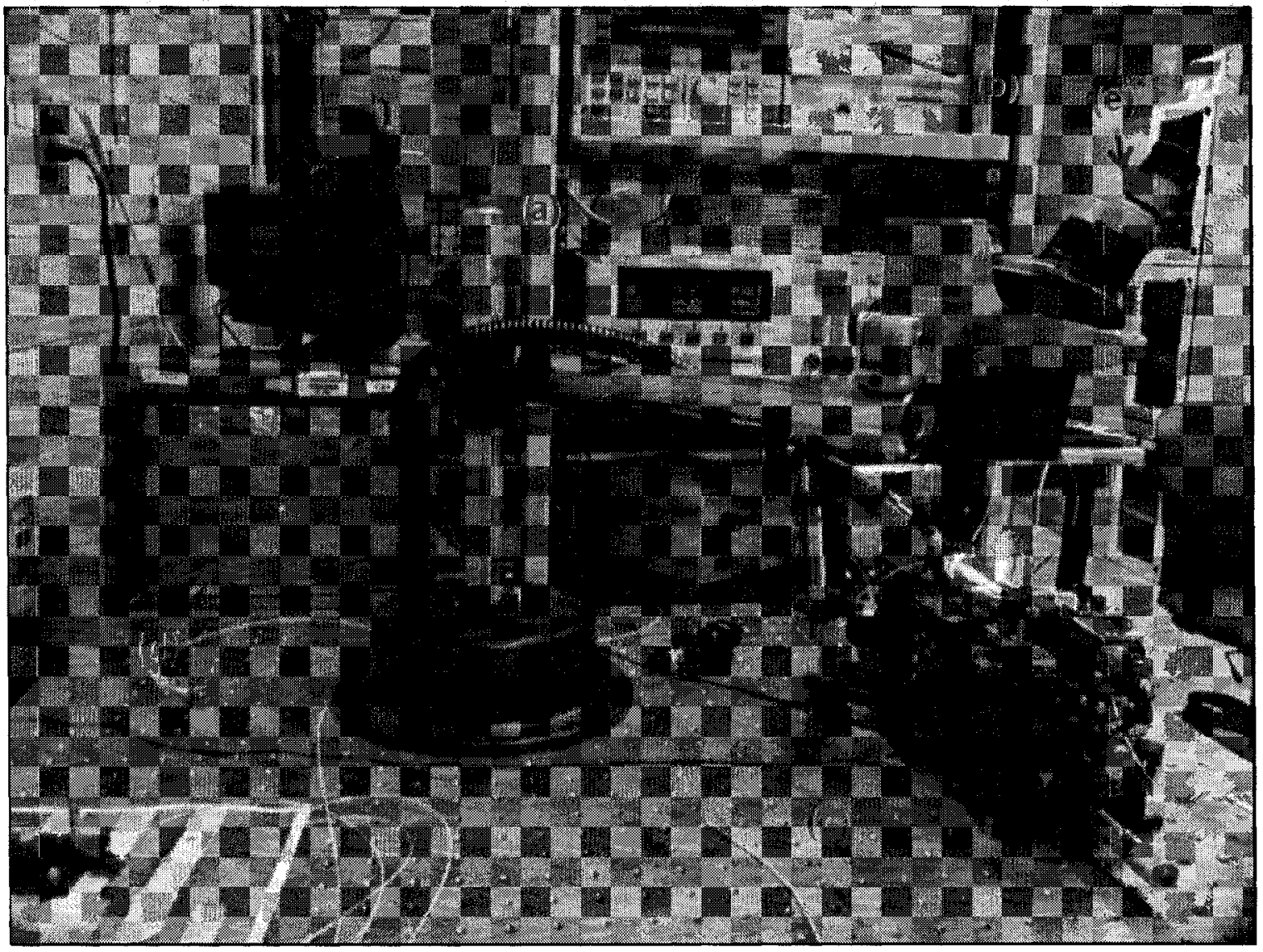

Figure 5.15. Carleton University's Measurement Setup. (a) Agilent/HP 8168F tunable laser source, (b) Agilent/HP 86142A optical spectrum analyzer, (c) test jig, (d) monitor showing waveguide output mode and (e) microscope for viewing fiber-sample-objective lens alignment. [Courtesy of Chris Raum]

Arrow (a) points to an Agilent/HP 8168F tunable laser source. It has a maximum output power of $3 \mathrm{~mW}$, and a wavelength range from $1460 \mathrm{~nm}$ to $1580 \mathrm{~nm}$. The output light from the laser is coupled to a tapered fiber from Corning®. This tapered fiber has a $3 \mu \mathrm{m}$ core size and $20 \mu \mathrm{m}$ focusing distance. Arrow (b) points to an Agilent/HP 86142A 
optical spectrum analyzer used to obtain the waveguide output. Arrow (c) points to the optical test jig. More will be discussed about this later. Arrow (d) points to a monitor that is connected to an IR camera mounted on the test jig. It shows the output optical mode of the J-coupler-PhQ system. Arrow (e) points to the microscope used to align the sample to the camera and fibers.

Figure 5.16 is a close up picture of the test jig setup. Three stages are placed close together and act as the input, sample, and output stages.

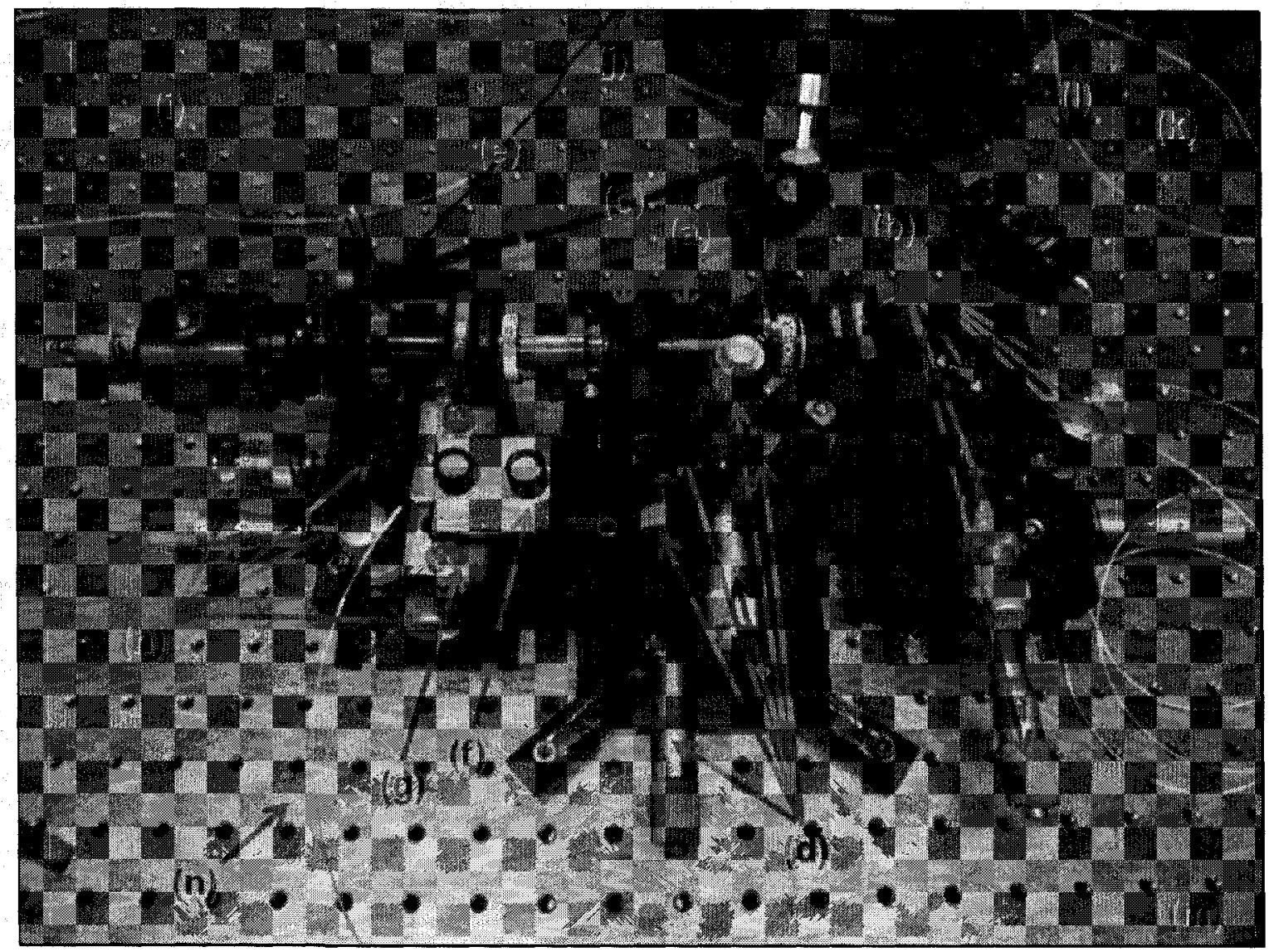

Figure 5.16. Close up picture of test jig. (a) Brass holder holds tapered fiber for input and output purposes, (b) stage that controls fine 3D motion and course 2D plane motion via knobs, (c) sample stage, (d) sample stage that controls 3D fine motion and course 1D motion (transverse to fiber), (e) 40x objective lens for output, (f) fiber stage which holds input tapered fiber, (g) camera-fiber stage for switching between camera and input fiber, (h) camera-input fiber stage and 3D fine motion and 1D course motion control knobs, (i) output IR camera connected to monitor and objective lens, (j) overhead broadband light source, (k) free space coupler, (l) wavelength filter, (m) tapered fiber for input/output purposes and (n) input tapered fiber. [Courtesy of Chris Raum] 
Arrow (a) points at the brass fiber hold that holds the Corning® tapered fiber and (b) points to the stage that controls this fiber. It points to $3 \mathrm{D}$ fine control knobs and $2 \mathrm{D}$ course control knobs. Arrow (c) points to where the sample would be placed for measurement and (d) points to the stage and control knobs of the sample stage. Arrow (e) points to the $40 \mathrm{X}$ output objective, and arrow (f) points to the fiber platform that holds the input fiber for coupling light in for OSA viewing. Arrow $(\mathrm{g})$ points to a custom made switchable platform that holds both the objective lens (e) and input fiber (f) platform. This switchable platform sits upon rollers for easy of exchange between the output mode (lens) and input mode (fiber). Arrow (h) points to the stage that holds this switchable platform. The stage has 3D fine control knobs and a 1D longitudinal course control that acts as a course focus for the objective. Arrow (i) points to an IR camera that is coupled directly to the video monitor. The sample platform is illuminated by a broadband light source pointed to by arrow (j). Arrow (k) and (l) points to a free space coupler and filter, respectively. This system is used for a super-continuum system which is not employed in these measurements. This system will be discussed further in Chapter 7 under the future work section. Arrows (m) and (n) point to the tapered fibers used in this setup.

\subsubsection{Measurement Process}

Measurements at Carleton were completed differently than those completed at NRC. The major difference was the absence of a photodetector at the Carleton setup. Instead, the output of the waveguides were coupled into a tapered fiber that was connected to the OSA. There was no optical-to-electrical conversion.

The sample was first placed on the sample stage and aligned with the lens, as shown in figure 5.17 . 


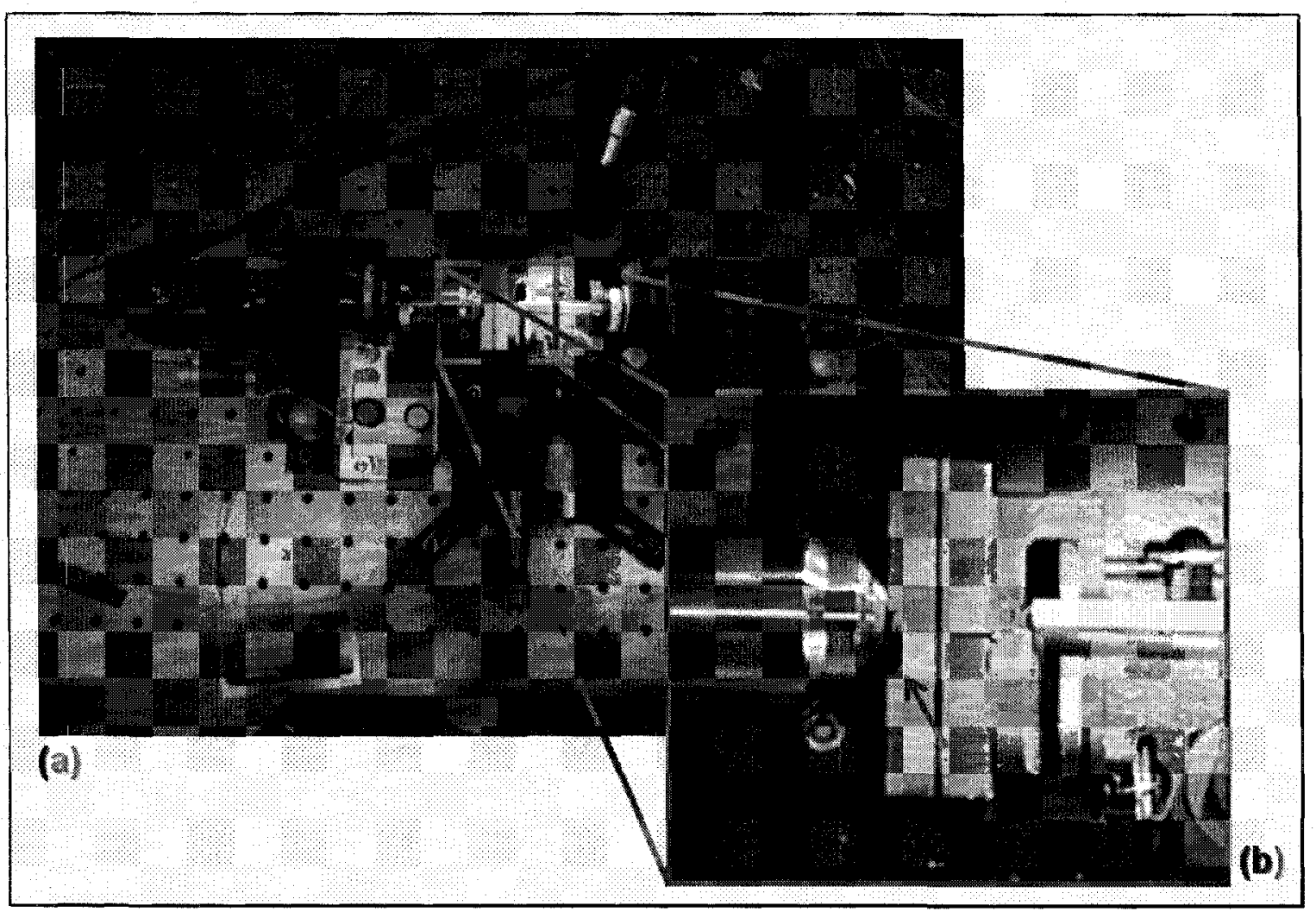

Figure 5.17. Positioning the Sample. (a) Overhead view of test jig and (b) close up of sample holder with sample (pointed to by arrow). [Courtesy of Chris Raum]

Light is then coupled from the fiber on the right in picture (b) of figure 5.17 to the waveguide. Careful position of all three stages (i.e. input fiber stage, sample stage, and lens stages) is required to obtain an optical mode through the waveguide. Figure 5.18 shows the results of careful positioning to obtain the output mode shown by the arrow in the monitor. The inset of figure 5.18 shows the third PhQ system to be measured.

Once the output mode is obtained, the input fiber that is connected to the tunable laser is disconnected from the tunable laser and connected to the OSA, thereby making it the output fiber. The roller stage holding the objective lens and fiber platform is adjusted so that the camera is slowly moved away from the former output end of the fiber, and the 
fiber platform is rolled in to become the input fiber. This new input fiber is connected to the tunable laser source. From this point, the new fiber input from the left is slowly brought in towards the waveguide and the OSA is closely monitored.

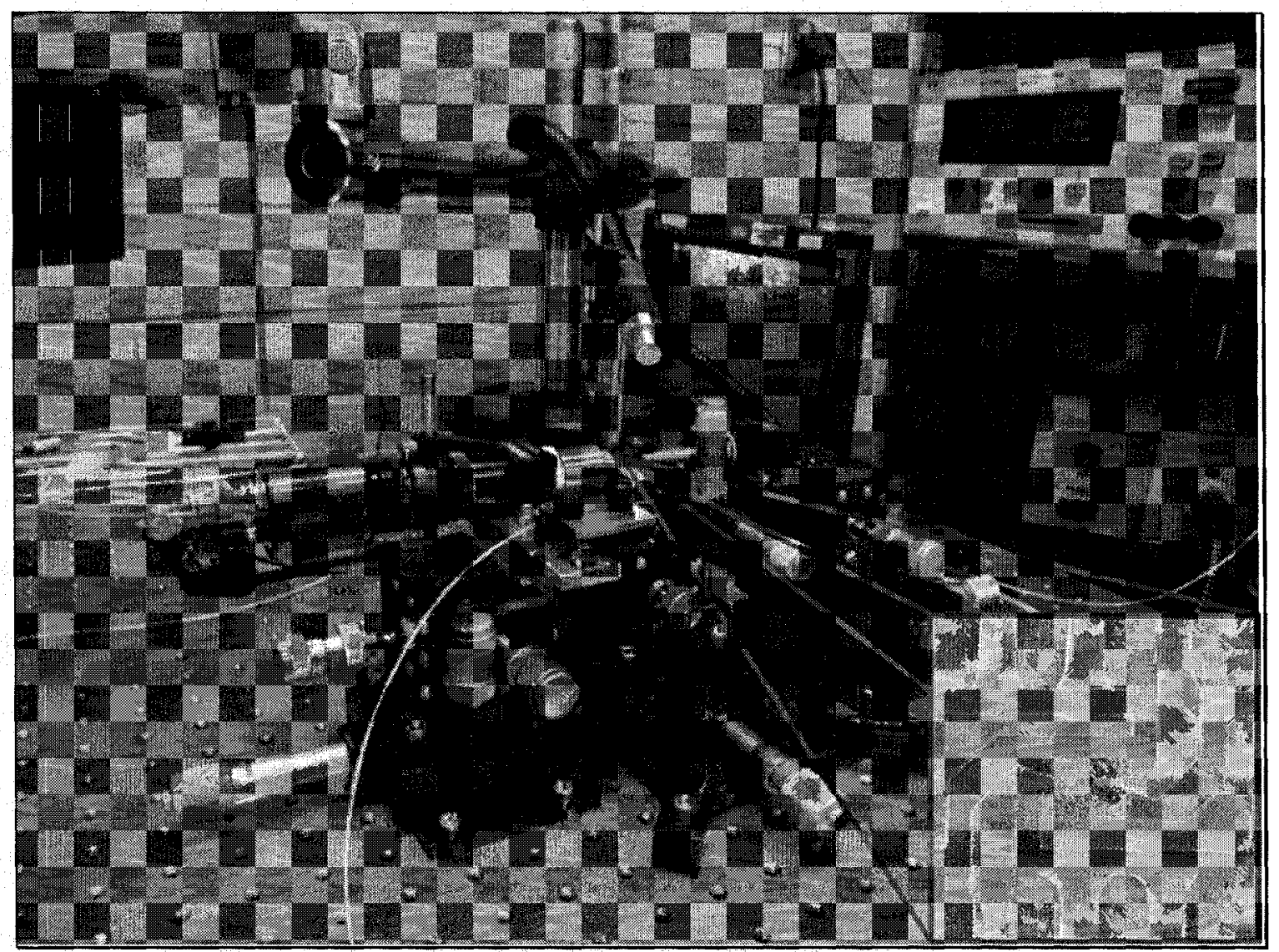

Figure 5.18. Result of careful adjustments. Arrow points to sharp images of waveguide mode of J-coupler-PhQ system. Inset is the third system to test, which is the J-couplerPhQ system. [Courtesy of Chris Raum] 


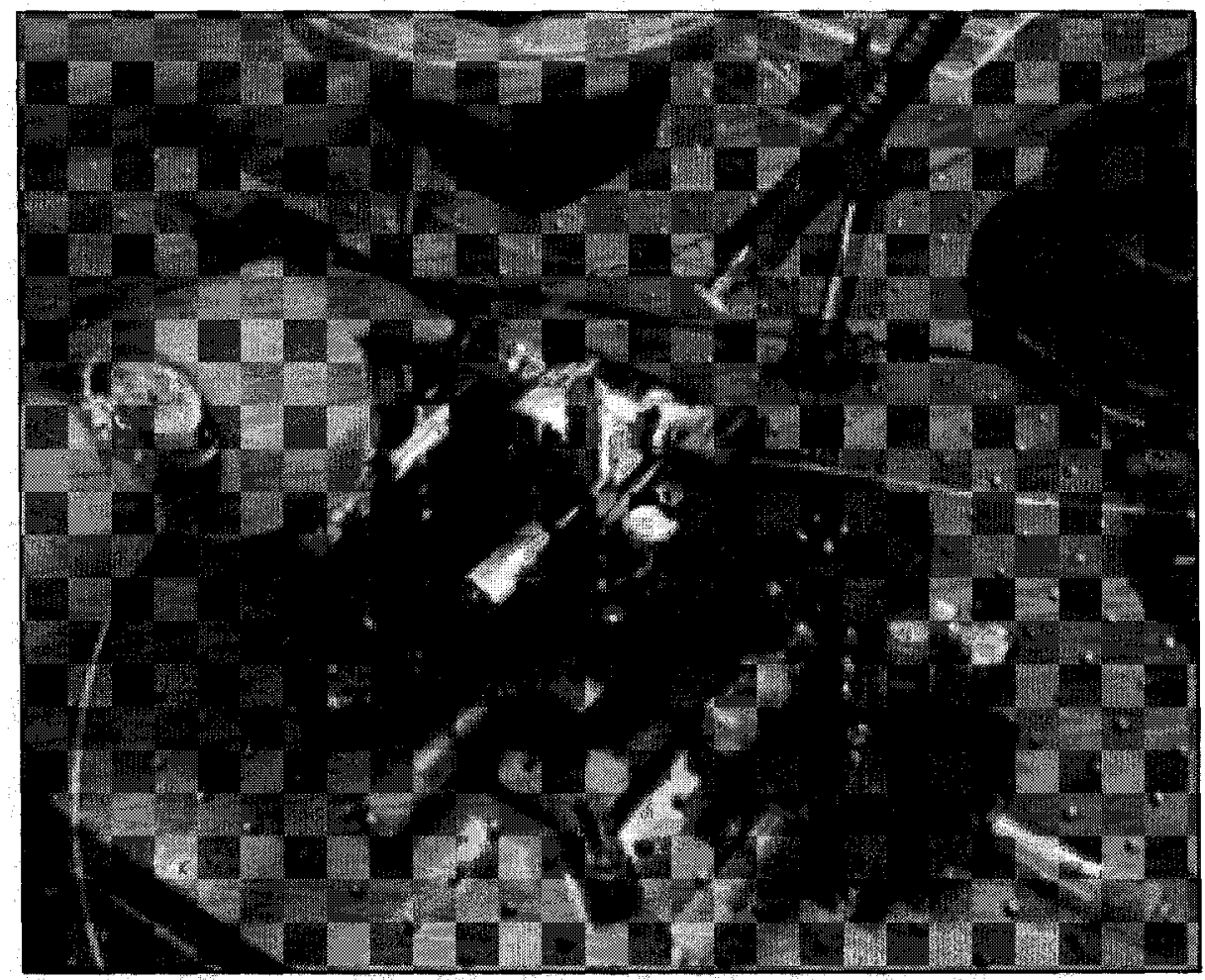

Figure 5.19. Moving lens away and bring new input fiber in one roller stage. Arrow indicates direction of motion. [Courtesy of Chris Raum]

\subsubsection{Measurement Results: System Three}

Using software written by Professor Gauthier and Scott Newman, the author was able to capture scan images from the OSA. Mr. Newman has written code for obtaining the peaks of the OSA output so that a wavelength scan of the output responses from any device can be recorded. Figure 5.20 shows the first scans of a PhQ crystal device measured at Carleton University. 


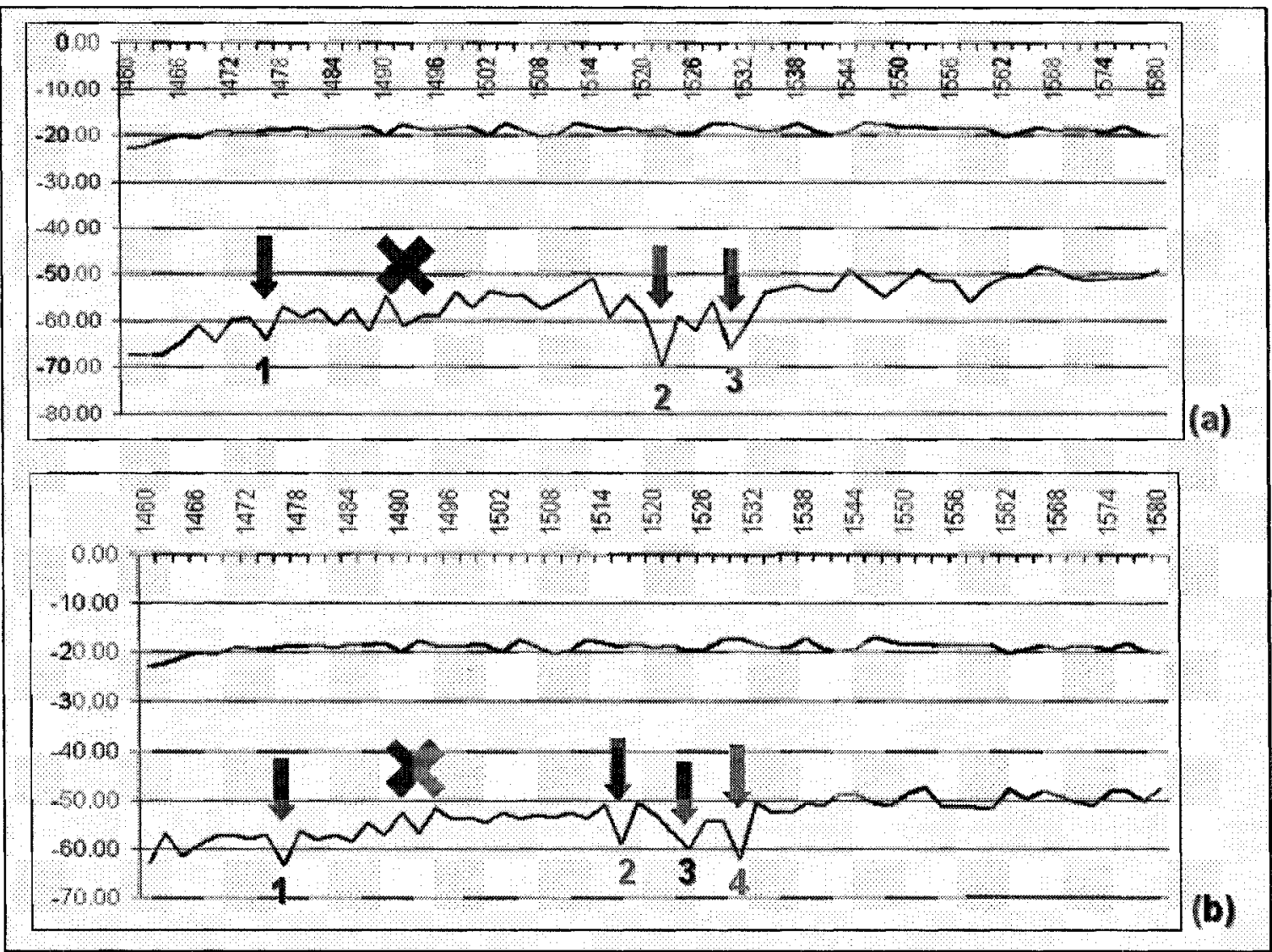

Figure 5.20. First scans done at Carleton for a PhQ device. Curves (a) and (b) show dips in generally the same area in the spectrum which are indicated by the numbered arrows. However, the predicated wavelengths from Chapter 3 do not show dips. The reason is that the scan resolution is very coarse, which is a $2 \mu \mathrm{m}$ set resolution.

In both scans there are consistent dip regions indicated by the numbered arrows. It appears that dip 3 of (a) and dip 4 of (b) are the same mode. Figure 5.21 shows a postmeasurement eigenmode analysis of the five modes that are associated with these dips. Mode (a) is associated with dip 1 of both scan (a) and scan (b). Mode (b) is associated with dip 2 of scan (a). Mode (c) is associated with both dip 3 of scan (a) and dip 4 of scan (b). Modes (d) and (e) are associated with dips 2 and 3, respectively. Each of these modes were generated by the different scatter probabilities available as the light passes through the structure. All these modes are of a $m=1$ nature. Most likely, the different states come from fewer degenerate states whose degeneracy has been lifted by the DFF of the structure. 


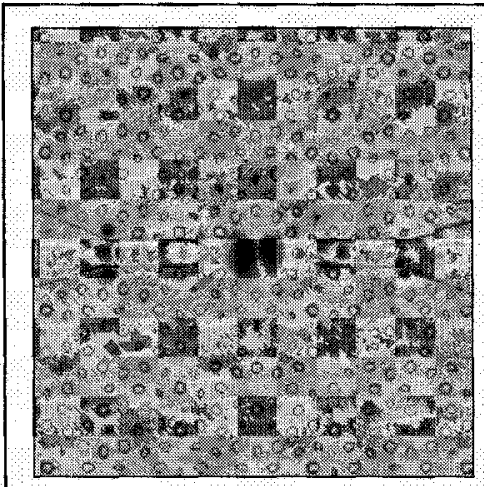

(a)

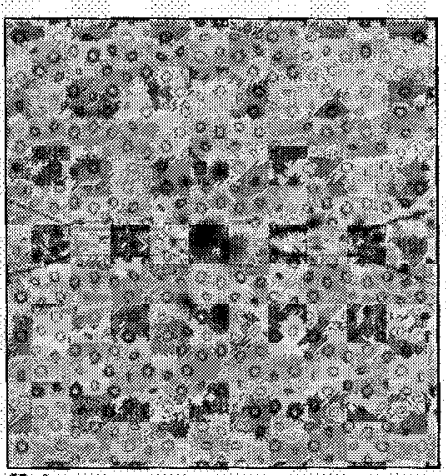

(b)



(c)

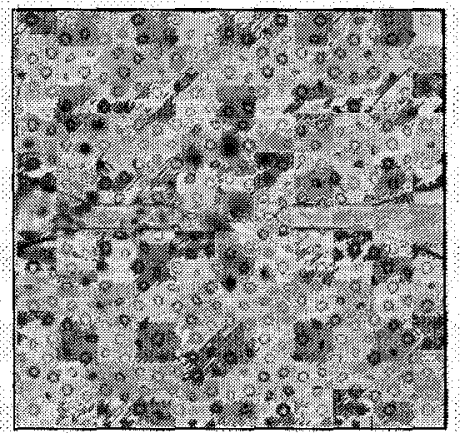

(d)

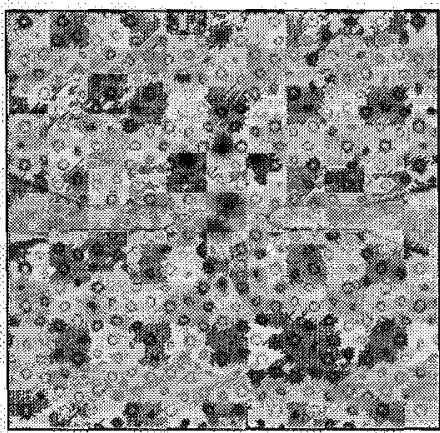

(e)

Figure 5.21. Post-measurement eigenvalue analysis of modes found in initial scan. (a) Dip 1 in both (a) and (b) scans, (b) dip 2 of (a) scan, (c) dip 3 of scan (a) and dip 4 of scan (b), modes (d) and (e) are at dips 2 and 3 of scan (b). These modes may be degenerate modes whose degeneracy is lifted by the DFF.

The $\mathrm{X}$ regions indicate no dips in the wavelengths that showed dips in the first two

structures measured. The reason for this may be that the scan rate was too coarse $(2 \mu \mathrm{m}$ per scan).

Figure 5.22 shows two higher resolution scans $(200 \mathrm{~nm})$ of the $\mathrm{X}$ region. It shows relatively good agreement with the predicated CAM state wavelengths of figure 3.53. 


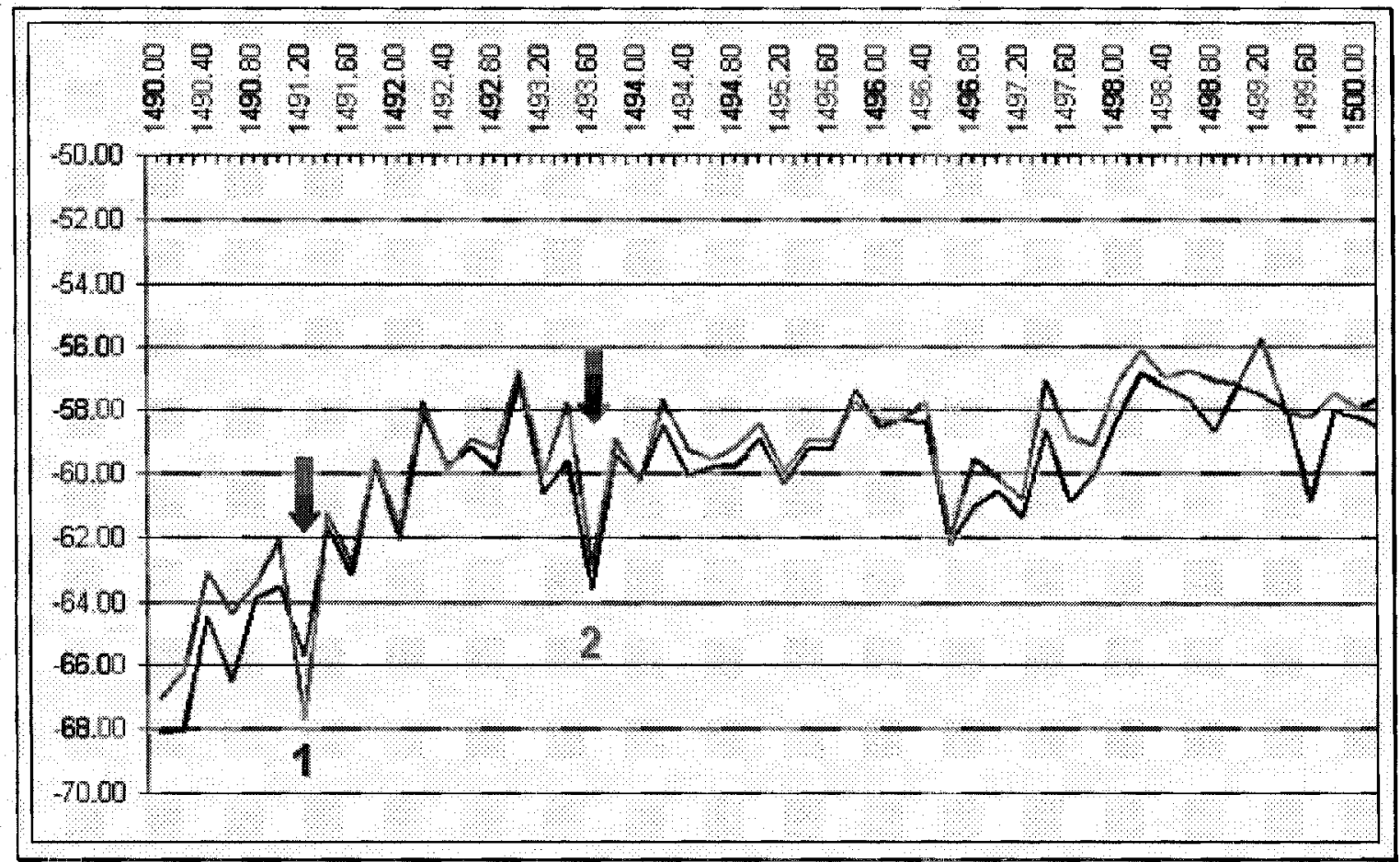

Figure 5.22. Two higher resolution scan of predication region. Dips 1 and 2 relatively correspond to the predicated CAM states that are Lamb shifted. The two scans overlapping in the right regions gives further evidence to the CAM state phenornena.

The wavelengths in dips 1 and 2 in figure 5.22 are relatively in the same order as the predicated eigenmodes shown in figure 3.53. Recall that these eigenmodes are the $m=0$ and $m=1$ modes. They appear to be in a Lamb shifted order because the $m=0$ mode is at a lower wavelength (i.e. higher energy) than the $m=1$ mode.

With the agreement of simulations and measurements demonstrated in this chapter, it would be beneficial to discuss these results and determine whether they support the theory put forward in this thesis about CAM states. The next chapter discusses these results and the implications of the theory of crystal angular momentum in PhQs. 


\section{Conclusions}

\subsection{Summary of Results}

Three PhQ systems were designed, fabricated, and tested for optical coupling to localized states generated by an inherent property of the quasicrystalline geometry. System one was a $10 \mu \mathrm{m} \times 10 \mu \mathrm{m}$ PhQ pattern (figure 4.30). System two was $40 \mu \mathrm{m} \times 20 \mu \mathrm{m}$ PhQ pattern (figure 4.31 ), and system three was $10 \mu \mathrm{m} \times 10 \mu \mathrm{m}$ PhQ pattern with $\mathrm{J}$-coupler ports (figure 4.32). System one's measurement response showed a large dip in transmission near the eigenvalue wavelength that was associated with an $m=1$ mode. These dips correspond to optical coupling from the waveguide or scattered light to the localized mode at the center of the pattern. The coupled light is then out-of-plane coupled as demonstrated by the IR images of figure 5.9. The dips seen here are very similar to dips seen elsewhere in the work on phononic (acoustic) quasicrystals [55].

System two was designed to repeat the findings of system one with an additional result: off-center localizations. These off-center results seem to indicate that local sites also have $m=0$-like and $m=1$-like localized modes. System three was designed to examine these localized modes further, and show that the predicted Lamb-shift $m=0$ and $m=1$ wavelengths were in fact present in the proper order of the physical response.

\subsection{Summary of Thesis}

The goal of this thesis was to explore the nature of localized modes in photonic quasicrystals and introduce the property of crystal angular momentum. The proposed and 
observed localized modes are regarded as the rotational equivalents of Bloch states in translationally symmetric systems. Various photonic quasicrystal structures with different dielectric form factors were examined through simulations. One particular photonic quasicrystal dielectric form factor yielded a series of localized states that resembled atomic angular momentum states about a central potential. The observed wavelength of the lowest order state was lower than the wavelength of the second lowest state. This result resembles the Lamb shift in atomic systems. Three photonic quasicrystal nanostructure systems were fabricated and measured to test the supposition. The experimental results of these three nanostructure systems agreed very strongly with the both a priori and a posteriori localized mode simulations. The agreement of the simulation and experimental results lead to the conclusion that crystal angular momentum in photonic quasicrystals is a real phenomena that can explain mode localization and bandstructure formation in photonic quasicrystals.

\subsection{Future Work}

Observation of the higher localized states $(m>1)$ was not possible because the expected wavelengths were the absorption range of the material used for the dielectric form factor. One suggestion for viewing these higher order modes is to scale the size of the structure as demonstrated by Wang et al [9]. Another suggestion is to explore other dielectric form factor configurations and different materials for the dielectric form factor such as a silicon-silica combination. 
The spectral range in this work was limited to the $\mathrm{S}$ and $\mathrm{C}$ bands (i.e. $1460 \mathrm{~nm}$ to $1580 \mathrm{~nm}$ ) of the optical communication channel. One could propose to use a strong broadband source to increase the spectral range, such as a supercontinuum setup.

From a fabrication point-of-view, the height of the rods in the photonic quasicrystals structures studied here were quite short. Fabricating taller rods would enhance Q-factors and increase the optical coupling of the observed modes. Creating taller nanoscale rods requires a study of hard marks and aspect ratio maintenance. Other processes such as LIGA could be explored.

This work also paves the way for possible theoretical applications that involve a tight-binding theory for light. Even though light does not form bound states, the Lamb shifted CAM states introduced here may lead a direction towards such a theory.

\subsection{Applications}

Areas of application can be split into two areas: tools for fundamental studies in physics and engineering devices. For the first area, the crystal angular momentum states of PhQs could be used in optical angular momentum experiments. PhQ systems can be ideal environments for optical spin-to-orbital angular momentum conversion [56]. Because the system has its own contribution to the conservation rules, one could, in effect, modify interactions by designing the PhQ structure. Another application in fundamental studies are matter-light interactions in quantum optics. Quantum dots in PhQs and interactions via crystal angular momentum may lead to tailoring a specification number of photon generation. This is ideal for single photon sources [57] used for quantum communication cryptography. Furthermore, there is growing interest in orbital angular momentum of 
light in parametric down-conversion for creating entangled photon states $[58,59]$. This can make applications such as quantum teleportation an everyday reality. One interesting application is accessing nonlinear qualities like frequency doubling and phase matching from the PhQ lattice qualities [60]. For device-oriented applications, these crystal angular momentum states may also lead to better cavity efficiencies for laser and LED devices [61,62]. Currently, papers in the literature have suggested using PhQs as adddrop multiplexers $[63,64]$. If the affect of crystal angular momentum is considered in these devices, perhaps better characteristics can be added and hence increase inefficiency and performance since, as this thesis shows, the PhQ itself is an intimate part of the conversion laws of the appropriate physics. 


\section{Appendices}

\section{Appendix I: Algebraic Vector Spaces}

\section{Algebraic Description}

The mathematical methods in physics are not only useful in providing a pathway from analytical equations to computer summations - they also provide powerful insight into what is physically happening and why it happens. The electric and magnetic field vector quantities are now seen as elements of an algebraic vector space. The technical definition of an algebraic vector space is an algebraic group of elements called vectors over an algebraic field of scalars. In most cases, the scalars are real; however, they can be complex*. In this particular case, the scalars are real because they represent frequency. Operators map vector space elements to other vector space elements. If the operator is other than the identity operator and the vector is not the null vector and it maps the vector element to a scaled version of itself, this is known as an eigenvalue problem. For example, if one has the following situation,

$$
\mathbf{A x}=\lambda \mathbf{x} \rightarrow(\mathbf{A}-\lambda \mathbf{I}) \mathbf{x}=0
$$

where $\mathbf{A}$ is some operator in a vector space, $\mathbf{I}$ is the identify operator, $\mathbf{x}$ is an element of the vector space, $\lambda$ is an element of the algebraic field discussed above, then in order for $\mathbf{x}$ to be other than the null vector, the determinant, which maps an operator to the real line, must be zero. This means that the operator $(\mathbf{A}-\lambda \mathbf{I})$ is not invertible, implying that

\footnotetext{
"Especially when one needs to perform integrations that are divergent.
} 
$\mathbf{x}=0 / 0$ which is not infinite or zero, but has some finite value. The characteristic ${ }^{*}$ equation,

$$
\operatorname{det}(\mathbf{A}-\lambda \mathbf{I})=0
$$

determines these finite values. Another important point is that to each element of a vector space is a dual element of a dual vector space. Dual elements map vector elements to the real line. The resultant number is usually called the inner product or dot product and is generally interrupted as a length or distance. The famous Dirac bra-ket notion, the bra is an element of the dual vector space and the ket is the element of the vector space. Hence a dot product between two vectors can be represented as,

$$
\mathbf{F} \cdot \mathbf{G} \equiv\langle\mathbf{F} \mid \mathbf{G}\rangle
$$

If the vector elements represent functions (scalar or vector functions), then the dot product is defined as,

$$
\langle\mathbf{F} \mid \mathbf{G}\rangle \equiv \int d^{3} r \mathbf{F}^{*}(\mathbf{r}) \cdot \mathbf{G}(\mathbf{r})
$$

Each operator in the vector space has an associated adjoint operator. If an operator maps kets to kets, the adjoint operator maps the associated bras to the associated bras. From the general property of inner products, one can define the form of the adjoint,

$$
\left\langle\psi^{\prime} \mid \varphi\right\rangle=\langle\mathbf{A} \psi \mid \varphi\rangle=\left\langle\psi\left|\mathbf{A}^{\dagger}\right| \varphi\right\rangle=\langle\varphi|\mathbf{A}| \psi\rangle^{*}=\langle\varphi \mid \mathbf{A} \psi\rangle^{*}=\left\langle\varphi \mid \psi^{\prime}\right\rangle^{*}
$$

A pictorial representation of this is shown in figure 0.1 . The adjoint can be equal to its operator. In this case, the operator is called Hermitian.

\footnotetext{
* The German word for characteristic is eigen, hence the name 'eigenvalue problems'.
} 


$$
\left\langle\psi^{\prime} \mid \varphi\right\rangle=\langle\mathbf{A} \psi \mid \varphi\rangle=\langle\psi|\mathbf{A}| \varphi\rangle=\langle\varphi|\mathbf{A}| \psi\rangle^{*}=\langle\varphi \mid \mathbf{A} \psi\rangle^{*}=\left\langle\varphi \mid \psi^{\prime}\right\rangle^{*}
$$

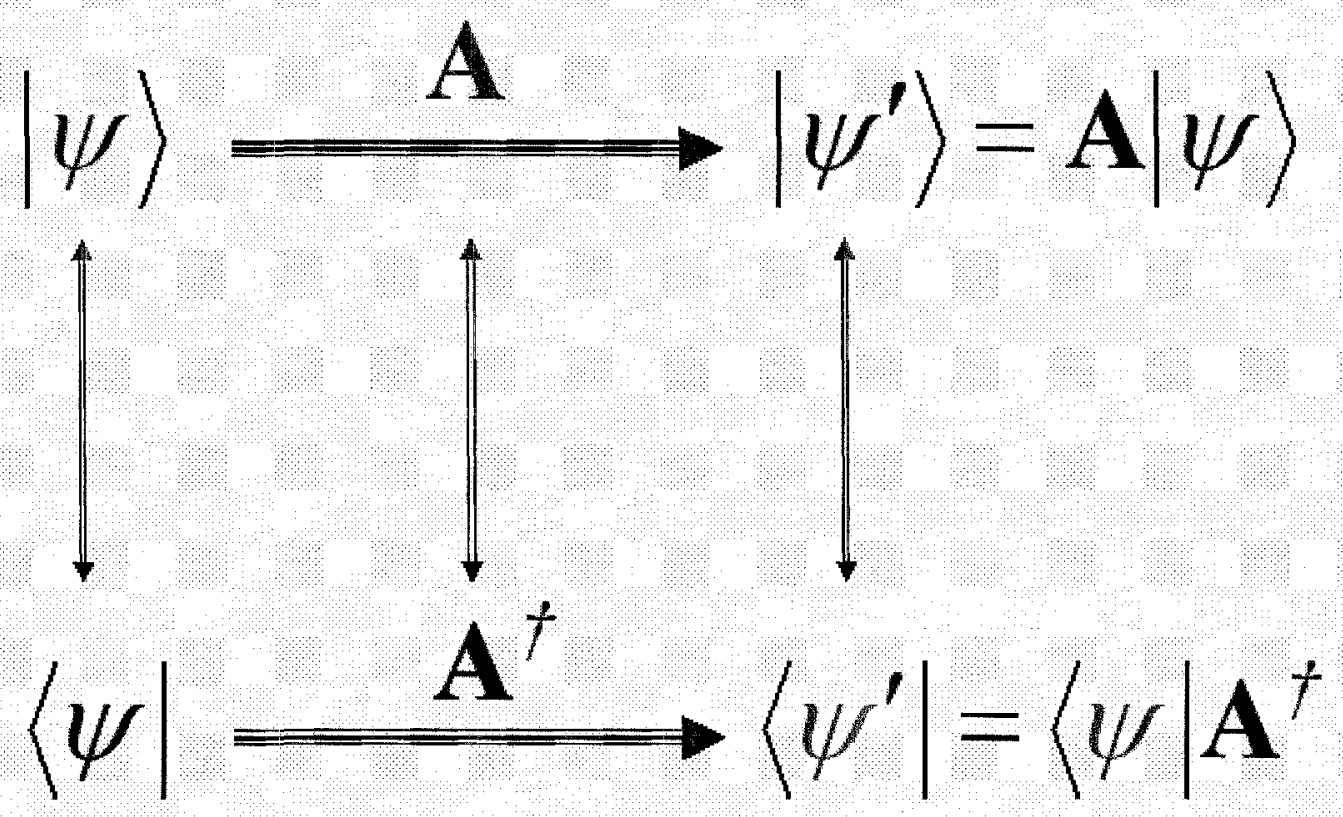

Figure 0.1. Correspondence between operator and its adjoint.

\section{Hermitian Operators}

Hermitian operators have very interesting eigenvalue properties. First, their eigenvalues are always real. This is important ${ }^{*}$ if these operators are to represent physical transformations (i.e. equations of motion). Second, and most importantly, the eigenvectors associated with their nondegenerate eigenvalues are always orthogonal. These means that the eigenvectors of Hermitian operators can be used as a basis in the vector space (or subspace). This means that Hermitian operators can represent spectra of discrete, continuous, or both discrete and continuous eigenvalues.

\footnotetext{
* Some authors in the literature have contested this point.
} 
It turns out that only one of the two operators defined above are Hermitian: the $L_{H}$ is Hermitian, but the $L_{E}$ is not. Although $L_{E}$ can be made Hermitian if its eigenvectors are scaled by $\sqrt{\varepsilon(\mathbf{r})}$ and the operator is,

$$
L_{E}^{\prime}=\frac{1}{\sqrt{\varepsilon(\mathbf{r})}} \nabla \times\left\{\nabla \times \frac{1}{\sqrt{\varepsilon(\mathbf{r})}}\right\}
$$

\section{Proof of Bloch's Theorem}

For electromagnetic radiation in crystals, solid-state physics provides the conservation rules, and electrodynamics determines the equations of motion. It is well known that when waves are confined with boundary conditions they become discrete in number. In addition, the number of allowed wavevectors in a primitive cell of the reciprocal lattice is equal to the number of sites in the crystal. Therefore, any function obeying these boundary conditions can be expanded via the allowed wavevectors. That is, the allowed wavevectors form a complete set within the primitive lattice,

$$
\psi(\mathbf{r}) \equiv\langle\mathbf{r} \mid \psi\rangle=\sum_{\mathbf{k}}\langle\mathbf{r} \mid \mathbf{k}\rangle\langle\mathbf{k} \mid \psi\rangle
$$

where $|\psi\rangle$ is a given state in vector space, $|\mathbf{r}\rangle$ is a well-defined state in position space, and $|\mathbf{k}\rangle$ are the complete wavevectors in the primitive cell. The amplitude for a state to be found in one of the wavevectors in the primitive cell can also be expressed by the complete set of allowable scattered vectors, via the Laue condition,

$$
\psi(\mathbf{r}) \equiv\langle\mathbf{r} \mid \psi\rangle=\sum_{\mathbf{k}}\langle\mathbf{r} \mid \mathbf{k}-\mathbf{K}\rangle\langle\mathbf{k}-\mathbf{K} \mid \psi\rangle
$$


The amplitude to scatter into an allowable wavevector in the primitive cell depends upon the amplitude interference of scattering into allowable wavevectors of other primitive cells. Next, it scatters through the crystal to the primitive cell in question,

$$
\psi(\mathbf{r}) \equiv\langle\mathbf{r} \mid \psi\rangle=\sum_{\mathbf{k}}\langle\mathbf{r} \mid \mathbf{k}-\mathbf{K}\rangle \sum_{\mathbf{K}^{\prime}}\left\langle\mathbf{k}-\mathbf{K}|H| \mathbf{k}-\mathbf{K}^{\prime}\right\rangle\left\langle\mathbf{k}-\mathbf{K}^{\prime} \mid \psi\right\rangle
$$

where $\left\langle\mathbf{k}-\mathbf{K}^{\prime} \mid \psi\right\rangle$ is the amplitude to scatter into an allowable scattered wavevector from another site, and,

$$
\left\langle\mathbf{k}-\mathbf{K}|H| \mathbf{k}-\mathbf{K}^{\prime}\right\rangle=E^{\prime} \mathbf{k}-\mathbf{K}\left|\mathbf{k}-\mathbf{K}^{\prime}\right\rangle \Rightarrow\left\langle\mathbf{k}-\mathbf{K}|(H-E)| \mathbf{k}-\mathbf{K}^{\prime}\right\rangle=0
$$

is the equation of motion, which would be Schrödinger's equation in electronic structures or Maxwell's wave equations in optical structures. What happens next is that the second summation in 0.10 is solved to find $\langle\mathbf{k}-\mathbf{K} \mid \psi\rangle$ and the solution is then put back into 0.11 , which can then be written as,

$$
\psi_{\mathbf{k}}(\mathbf{r})=\sum_{\mathbf{K}} c_{\mathbf{k}-\mathbf{K}} e^{i(\mathbf{k}-\mathbf{K}) \cdot \mathbf{r}}=e^{i \mathbf{k} \cdot \mathbf{r}}\left(\sum_{\mathbf{K}} c_{\mathbf{k}-\mathbf{K}} e^{-i \mathbf{K} \cdot \mathbf{r}}\right)=e^{i \mathbf{k} \cdot \mathbf{r}} u_{\mathbf{k}}(\mathbf{r})
$$

where $c_{\mathbf{k}-\mathbf{K}} \equiv\langle\mathbf{k}-\mathbf{K} \mid \psi\rangle$. This is the proof of Bloch's theorem. Now, if

$$
\begin{aligned}
\psi_{\mathbf{k}}(\mathbf{r}+\mathbf{R}) & =e^{i \mathbf{k} \cdot(\mathbf{r}+\mathbf{R})} u_{\mathbf{k}}(\mathbf{r}+\mathbf{R}) \\
& =e^{i \mathbf{k} \cdot \mathbf{R}} e^{i \mathbf{k} \cdot \mathbf{r}} u_{\mathbf{k}}(\mathbf{r}) \\
& =e^{i \mathbf{k} \cdot \mathbf{R}} \psi_{\mathbf{k}}(\mathbf{r})
\end{aligned}
$$

This result shows that a discrete symmetry operation, such as a spatial translation via a lattice vector, is equal to multiplication by a complex exponential. This result is similar to continuous transformations, as will be shown later in the thesis. It even lends itself to the explanation that the eigenvectors of the discrete translation operator commute 
with the eigenvectors of the equation-of-motion operator. This implies that the two operators possess a complete set of common eigenvectors. However, the point that must be made here is that this result is non-rigorous from a mathematical point of view. It is non-rigorous from a group theorctical point of view because discrete symmetries cannot be described via Lie algebra. This point is being made now because later when only rotational symmetries are available in the system, a similar approach will be asserted, namely, that the rotational symmetry gives rise to a Bloch-type modes about the center of rotation. This rotational Bloch equivalent looks like a defect mode but is fundamentally different. 


\section{Appendix II: Green's Functions}

The origin of Green functions stems from boundary conditions. In translationally periodic materials, the boundary conditions are handled in an abstract manner rather than in a direct way. As discussed above, the periodic boundary conditions in real space lead to confined boundary conditions in momentum-space resulting in discrete eigenvalues. The physical boundary conditions in position-space lead to discontinuities in the dispersion relation. Hence, it is the abstracted manner in how the boundary conditions in position and momentum-space are handled that leads to the bandstructure picture in translationally symmetric structures. PhQs and disordered media are not translationally symmetric so the boundary conditions cannot be handled in this abstracted manner. If one removes all boundary conditions, then all that remains are equations of motion. In this case electromagnetism, it is the wave equations. To handle boundary conditions generically, one then uses mathematical statements that relate closed regions to their bounding surfaces. There is a mathematical theorem known as the fundamental theorem of exterior calculus that states that if one has a $p$-form ${ }^{*} \varphi$,

$$
\int_{R} d \varphi=\oint_{\partial R} \varphi
$$

Where $R$ is some compact $(p+1)$-dimensional (oriented) region whose (oriented) $p$ dimensional boundary (also compact) is denoted $\partial R$. This theorem in regular 3-space gives rise to the famous divergence theorem of Gauss $(p-2)$ and Stokes theorem $(p=1)$. Staring with the 3 -space divergence theorem,

\footnotetext{
*An $n-p$ dimensional function that maps $n$-dimensional vectors to the real line or complex plane.
} 


$$
\int(\nabla \cdot \mathbf{A}) d^{3} x=\oint_{j} \mathbf{A} \cdot \mathbf{n} d a
$$

where $\mathbf{n}$ is the normal vector of the surface and $\mathbf{A}$ is a well-behaved vector field (goes to zero at infinity and is continuous differentiable - what mathematician call $C^{\infty}$ functions) defined in a volume $V$ bounded by a closed surface $S$. Let $\mathbf{A}=\phi \nabla \psi$, where $\psi$ and $\phi$ are arbitrary scalar fields. If the divergence theorem is then expressed in terms of these arbitrary scalar fields one has,

$$
\int_{d}\left(\phi \nabla^{2} \psi+\nabla \phi \cdot \nabla \psi\right) d^{3} x=\oint_{S} \phi \frac{\partial \psi}{\partial n} d a
$$

where $\phi(\partial \psi / \partial n) \equiv \phi \nabla \psi \cdot \mathbf{n}$. Equation 0.16 is known as Green's first identity. If $\mathbf{A}$ is restated as $\mathbf{A}=\psi \nabla \phi$, inserted in the divergence theorem again and then subtracted from 0.16 , one obtains Green's second identity,

$$
\int\left(\phi \nabla^{2} \psi+\psi \nabla^{2} \phi\right) d^{3} x=\oint_{S}\left[\phi \frac{\partial \psi}{\partial n}-\psi \frac{\partial \phi}{\partial n}\right] d a .
$$

This is a non-physical statement because of the arbitrary specification of the scalar fields and their normal derivatives. This mathematical statement can be "tailored" to a physical situation with an appropriate choice of scalar function. In vector problems representing reality, scalars, called potentials, can be deduced via vector identities. They must also be gauge invariant if they are to represent physical reality. The gauge invariance allows a degree of freedom in choosing an appropriate potential that can he exploited to remove either the scalar field or its normal derivative on the right hand side of 0.17 . One last point must be made about the choice of potentials that will make 0.17 into a physical statement. A differential operator statement, such as, 


$$
L y(x)=f(x)
$$

where $L$ is a differential operator in state space. Differential operator statements can be interpreted as mappings from possible responses of the system to possible sources. Integral operators, $K$, statements say the inverse: mapping a source to a possible response of the system,

$$
y(x)=K f(x) \equiv\langle x|K| f\rangle=\int d x^{\prime}\left\langle x|K| x^{\prime}\right\rangle\left\langle x^{\prime} \mid f\right\rangle \equiv \int d x^{\prime} G\left(x, x^{\prime}\right) f\left(x^{\prime}\right)
$$

where $G\left(x, x^{\prime}\right)$ is called the kernel of the integral operator. It seems that the integral operator is the inverse operator of the differential operator. Both operators can describe a system, and if the differential operator's eigenvectors are complete, the integral and differential operators applied consecutively represent unity. In position space, an amplitude that ties one position to another is represented via a Dirac function,

$$
\left\langle x^{\prime} \mid x\right\rangle=\delta\left(x-x^{\prime}\right)
$$

If one has a position state, multiplies by one, expands using the completeness theorem of position space, uses 0.19 , one can show the condition this kernel must fulfill,

$$
|x\rangle=L K|x\rangle=\int L\left|x^{\prime}\right\rangle\left\langle x^{\prime}|K| x\right\rangle=\int\left|x^{\prime}\right\rangle L G\left(x^{\prime}, x\right)=\int\left|x^{\prime}\right\rangle\left\langle x^{\prime} \mid x\right\rangle=|x\rangle
$$

which means,

$$
L G\left(x^{\prime}, x\right)=\delta\left(x-x^{\prime}\right)
$$

This shows that the kernel, $G\left(x^{\prime}, x\right)$, of an integral operator, which is the inverse operator of $L$, is a distribution $\left(C^{-\infty}\right)$, meaning that it is a dual element of a vector space of well-behaved functions $\left(C^{\infty}\right)$. It also shows that $L$ is a self-adjoint operator since it 
operates in both vector spaces. Knowing that $G\left(x^{\prime}, x\right)$ is a distribution rather than a regular function is important in describing quasicrystals and disorder medium.

The differential operator in 0.17 is $L=\nabla^{2}$. Now, in order for 0.17 to be a physical statement, the kernel associated with $\nabla^{2}$ must be found, and in addition be modified to remove either the scalar field or its normal derivative on the right hand side of 0.17 . When this is done, the kernel is referred to as a Green function. Hence, Green functions are generally not unique, and require specifications of symmetry, boundary conditions, and other externally imposed criteria to make them unique. This is why finding the appropriate Green function is difficult, if not impossible. However, a perturbative approach can be applied when a simple geometry is first solved for then changed slowly to fit the actual physical situation.

If it is so difficult to find the Green functions, they why try to find it?

\section{Density of States from Green's Functions}

The Green function has some very lucrative properties. First, it solves the problem completely. What does this mean? Any input can be resolved in terms of Dirac delta functions. Some mathematicians argued that the Dirac delta function is not a true function. However, with the introduction of hyperfunctions by Mikio Sato and notions like Rigged Hilbert space that claim has less value. Although there are still problems in defining multiplication of delta functions which bring about many headaches for particle theorists, delta functions can resolve any practical input function. Practically, the delta function represents an impulse to the system. The system responds to this in a characteristic way. If the input is "chopped" up into impulse then output is continuously added in an appropriate manner (i.e. convolution), and then finally a complete response is 
obtained. Second, it provides a complete picture of the density of states within a structure. This is illustrated in the following equation. Suppose one starts with a Green's function relation in position-space,

$$
[z-L] K(z)=1
$$

where the differential operator is written in a Helmholtz form, and the integral operator shows an explicit dependence upon some complex number. Now, if the differential operator is Hermitian and has a complete set of discrete and/or continuous eigenvectors,

$$
K(z)=\frac{1}{[z-L]}=\sum_{n} \frac{\left|\phi_{n}\right\rangle\left\langle\phi_{n}\right|}{[z-L]}+\int d c \frac{\left|\phi_{c}\right\rangle\left\langle\phi_{c}\right|}{[z-L]}=\sum_{n} \frac{\left|\phi_{n}\right\rangle\left\langle\phi_{n}\right|}{z-\lambda_{n}}+\int d c \frac{\left|\phi_{c}\right\rangle\left\langle\phi_{c}\right|}{\left[z-\lambda_{c}\right]}
$$

Projecting this back into position space,

$$
\left\langle\mathbf{x}|K(z)| \mathbf{x}^{\prime}\right\rangle \equiv G\left(\mathbf{x}, \mathbf{x}^{\prime}, z\right)=\sum_{n} \frac{\phi_{n}(\mathbf{x}) \phi_{n}^{*}\left(\mathbf{x}^{\prime}\right)}{z-\lambda_{n}}+\int d c \frac{\phi_{c}(\mathbf{x}) \phi_{c}^{*}\left(\mathbf{x}^{\prime}\right)}{\left[z-\lambda_{c}\right]} .
$$

Because $L$ is Hermitian, all of its eigenvalues are real. Hence, if $\operatorname{Im}\{z\} \neq 0$ then $z \neq\left\{\lambda_{n}\right\}$, which means that $G\left(\mathbf{x}, \mathbf{x}^{\prime}, z\right)$ is an analytic function in the complex $z$-plane except at those points or portions of the real $z$-axis that correspond to the eigenvalues of $L$. $G\left(\mathbf{x}, \mathbf{x}^{\prime}, z\right)$ exhibits simple poles at the position of discrete eigenvalues of $L$. The inverse of this statement is also true: the poles of $G\left(\mathbf{x}, \mathbf{x}^{\prime}, z\right)$ give the discrete eigenvalues of $L$. If $z=\lambda$, where $\lambda$ belongs to the continuous spectrum of $L$, one has to express the Green function in a limiting process. If the continuous spectrum is associated with propagating or extended states (eigenstate does not decay as $x \rightarrow \infty$ ), the side limits of 
$G\left(\mathbf{x}, \mathbf{x}^{\prime}, \lambda \pm i s\right)$ as $s \rightarrow 0^{+}$exist but are different from each other. This type of continuous spectrum results in a branch cut $^{*}$ in $G\left(\mathbf{x}, \mathbf{x}^{\prime}, z\right)$ along part(s) of the real $z$-axis. Hence,

$$
G^{ \pm}\left(\mathbf{x}, \mathbf{x}^{\prime}, \lambda\right) \equiv \lim _{s \rightarrow 0^{+}} G^{ \pm}\left(\mathbf{x}, \mathbf{x}^{\prime}, \lambda \pm i s\right) .
$$

This form of the Green function allows one to use the following identity,

$$
\lim _{y \rightarrow 0^{+}} \frac{1}{x \pm i y}=\mathrm{P} \frac{1}{x} \mp i \pi \delta(x)
$$

where $\mathrm{P}$ here means taking the principle value. It turns out after some math that density of states is related to the imaginary part of the trace of the Green function (i.e. local density of states):

$$
N(\lambda)=\mp \frac{1}{\pi} \operatorname{Im}\left\{\operatorname{Tr}\left\{G^{ \pm}(\lambda)\right\}\right\} .
$$

This result shows the strength in finding the Greens function for any system configuration. However, finding the Greens function becomes very difficult if the system understudy is complicated. When it comes to PhCs and PhQs, not only are the lattices important in determining the bandstructure, but so are the form factors of the dielectric structure around each lattice point. Many papers have been published to study band formation in photonic crystals and photonic quasicrystals using Greens functions that have circular form factors, however, none has studied structures that have non-circular or complicated form factors. The main topic of this thesis is PhQs that have non-standard form factors. The method of analysis will be numerical, but the theory of Greens

\footnotetext{
"Branch cuts in a domain represents 'multivaluedness' in the domain.
} 
functions has been discussed since it may be the only way forward to a more theoretically rigorous theory. 


\section{Appendix III: Diffusion Picture versus Wave Picture}

How is the diffusive behavior of waves modeled theoretically? This is a vast and complicated topic, which means that it will not be covered entirely here, although it is worth showing a few theoretical results to get an appreciation of the path to localization of disordered media. First, one has the general form of the wave equation,

$$
H \varphi=\left(\nabla^{2}+\kappa^{2}\right) \varphi(\mathbf{r})=0
$$

where $H \equiv\left(\nabla^{2}+\kappa^{2}\right)$ is the Helmholtz operator, $\kappa$ is the term representing the potential and $\varphi(\mathbf{r})$ is the wave function. The potential term can be separated into a spatial dependent and independent term,

$$
\kappa^{2}=\kappa_{0}^{2}-\sigma(\mathbf{r})
$$

where $\kappa_{0}=\omega / v_{0}$ and

$$
\sigma(\mathbf{r})=[1-\varepsilon(\mathbf{r})] \frac{\omega^{2}}{v_{0}^{2}}
$$

where $\varepsilon(\mathbf{r})$ is the inhomogeneous dielectric function. The assumption here is that $\varepsilon(\mathbf{r})$ is not spatially periodic. A complete solution of 0.29 involves finding the Green's function,

$$
\left(\nabla^{2}+\kappa^{2}\right) G\left(\omega, \mathbf{r}, \mathbf{r}^{\prime}\right)=\delta\left(\mathbf{r}-\mathbf{r}^{\prime}\right)
$$

where $\mathbf{r}$ is the point of interest and $\mathbf{r}^{\prime}$ is the source point. One has to note that the source point position is an important parameter in disordered structures because the response to a point source will depends on its position. When dealing with complicated media, the general approach is to start with a simple structure then move on to the complicated 
media. In this case, one starts with a homogenous medium. This also means that the system response is now independent of the source position. The Green's function depends only on the relative separation $\mathbf{r}-\mathbf{r}^{\prime}$ between the source and detector. Hence,

$$
\left(\nabla^{2}+\kappa_{0}^{2}\right) G\left(\omega_{0}, \mathbf{r}-\mathbf{r}^{\prime}\right)=\delta\left(\mathbf{r}-\mathbf{r}^{\prime}\right)
$$

To begin analyzing a material that is disordered, it makes sense to start with an ordered lattice and then add randomization to the lattice. This is done when the Green's function takes the form,

$$
G\left(\omega_{0}, \mathbf{k}\right) \propto \frac{1}{\kappa_{0}^{2}-\beta e(\mathbf{k})} .
$$

where $G\left(\omega_{0}, \mathbf{k}\right)$ is the spatial Fourier transform of $G\left(\omega_{0}, \mathbf{r}-\mathbf{r}^{\prime}\right), e(\mathbf{k})$ is the eigenvalues of a discrete ordered lattice, and $\beta$ is a randomization factor.

To move to the diffusion of light, one first needs the diffusion equation,

$$
\frac{\partial \phi}{\partial t}=D \nabla^{2} \phi(\mathbf{r}, t)
$$

where this time $\phi$ is the density of the diffusion material and $D$ is the diffusion constant which is usually a fixed physical property of the medium. It is known that light and photon diffusion exist and it would be beneficial to describe this theoretically.

Diffusion results from the statistical character of $\mathbf{r}$, which is not directly evident from $\mathbf{r}$. In order to extract the diffusive behavior, one needs to take the moments of $\mathbf{r}-$ i.e. $\langle\mathbf{r}\rangle,\left\langle\mathbf{r}^{2}\right\rangle$, etc. Here, the angular brackets denote averaging over different configurations of the random perturbations $\sigma(\mathbf{r})$ in the wave scattering case. The same approach is applied to the homogenous Green's function. One would find moments of 
the Green's function $\langle G\rangle$. This moment gives the wave characteristics in an averaged sense. It also gives the spatial scale beyond which the average sense does not work anymore. This means that $\langle G\rangle \rightarrow 0$. However, this does not mean that the wave is localized. What it does mean is that the wave is losing its coherence. Transport characteristics of the wave are described via the second moment, $\left\langle G G^{*}\right\rangle$. This moment defines the diffusion constant of a wave in a disordered media. To obtain the phase interference effects that lead to non-classical diffusion effect, the fourth moment is required, $\left\langle G G^{*} G G^{*}\right\rangle$, which is the topic of the next section. 


\section{Appendix IV: Noether's theorem}

A formal proof of Noether's theorem is beyond the scope of this thesis but it is worth showing how this link between symmetry and dynamical variables comes about*. Noether's theorem is derived from Hamilton's first principle, namely that the action of a system is an extremum. In concise form, Hamilton's first principle is stated:

$$
\delta S=0
$$

where $S$ is integral of the Lagrangian,

$$
S=\int d^{4} x L\left(\psi^{\alpha}(x), \partial_{\mu} \psi^{\alpha}(x)\right)
$$

where $L\left(\psi^{\alpha}(x), \partial_{\mu} \psi^{\alpha}(x)\right)$ is the Lagrangian. Formally, it is known as the Lagrangian density because its arguments are field quantities, in this case, the 4-vector $\psi^{\alpha}$ and its 4derivative $\partial_{\mu} \psi^{\alpha}$. Applying 0.36 to 0.37 yields the famous Euler-Lagrange equation, which is normally called the equation of motion. If an infinitesimal transformation is applied to the field quantities,

$$
\psi^{\alpha}(x) \rightarrow \psi^{\prime \alpha}(x)=\psi^{\alpha}(x)+\alpha \Delta \psi^{\alpha}(x)
$$

where $\alpha$ is an infinitesimal parameter and $\Delta \psi^{\alpha}(x)$ is some distortion of the field configuration, and the equations of motions are left invariant, then this transformation is called a symmetry. Because the Euler-Lagrange equation would be left invariant with this transformation, the Lagrangian must also be invariant, up to a 4-divergence:

\footnotetext{
* It is important that something of this be shown in this work since, first, it is the theoretical inspiration of this work and, second, the author should show some proficiency in this area.
} 


$$
L \rightarrow L+\infty \partial_{\mu} J^{\mu}
$$

for some $J^{\mu}$. When comparing the distortion in the Lagrangian to the result obtained by varying its arguments,

$$
\begin{aligned}
\alpha \Delta L & =\frac{\partial L}{\partial \psi}(\alpha \Delta \psi)+\frac{\partial L}{\partial\left(\partial_{\mu} \psi\right)} \partial_{\mu}(\alpha \Delta \psi) \\
& =\alpha \partial_{\mu}\left(\frac{\partial L}{\partial\left(\partial_{\mu} \psi\right)} \Delta \psi\right)+\alpha\left[\frac{\partial L}{\partial \psi}-\partial_{\mu}\left(\frac{\partial L}{\partial \partial_{\mu} \psi}\right)\right] \Delta \psi
\end{aligned}
$$

and then applying the Euler-Lagrange equation to the second term, one finally gets:

$$
\partial_{\mu} j^{\mu}=0
$$

where

$$
j^{\mu}(x)=\frac{\partial L}{\partial\left(\partial_{\mu} \psi\right)} \Delta \psi-J^{\mu}
$$

Equation 0.41 states that the current $j^{\mu}(x)$ is conserved. For each continuous symmetry of $L$, there is a conservation law. As a vivid example, the Lagrangian is purely kinetic, $L=\frac{1}{2}\left(\partial_{\mu} \psi\right)^{2}$. The transformation $\psi \rightarrow \psi+\alpha$ leaves $L$ unchanged, so the current $j^{\mu}=\partial^{\mu} \psi$ is conserved (i.e. field momentum is conserved). 


\section{Appendix V: Gauge Invariance and the Electromagnetic Field}

One now introduces the covariant and contravariant ${ }^{*}$ notations of the 4-vector (Minkowski space notation), respectively,

$$
\begin{aligned}
& A^{\mu}=(\Phi, \mathbf{A}) \\
& A_{\mu}=(\Phi,-\mathbf{A}) .
\end{aligned}
$$

and the covariant and contravariant notation of the 4-current,

$$
\begin{aligned}
& j_{E M}^{\mu}=\left(\rho_{E M}, \mathbf{j}_{E M}\right) \\
& j_{\mu}^{E M}=\left(\rho_{E M},-\mathbf{j}_{E M}\right)
\end{aligned}
$$

Differential operators are represented in a similar compact form,

$$
\begin{gathered}
\partial^{\mu}=\left(\partial_{t},-\nabla\right) \\
\partial_{\mu}=\left(\partial_{t}, \nabla\right) . \\
\partial_{\mu} \partial^{\mu} \equiv \partial_{t}^{2}-\nabla^{2} .
\end{gathered}
$$

The continuity relation is then written as,

$$
\frac{\partial \rho_{E M}}{\partial t}=\nabla \cdot \mathbf{j}_{E M} \rightarrow \frac{\partial \rho_{E M}}{\partial t}-\nabla \cdot \mathbf{j}_{E M}=0 \rightarrow \partial_{\mu} j_{E M}^{\mu}=0
$$

which states the 4-current $j_{E M}^{\mu}$ is a conserved quantity. In 3-space problems, the potentials are found to decouple the Maxwell equations into two uncoupled sccond-order differential equations,

\footnotetext{
* Covariant and contravariant with respect to the basis vectors of the space. Covariant vectors transform like the basis vectors and contravariant vectors transform in an inverse manner when the basis vectors are transformed.
} 


$$
\begin{gathered}
\nabla^{2} \Phi-\frac{1}{c^{2}} \frac{\partial^{2} \Phi}{\partial t^{2}}=-\frac{\rho}{\varepsilon_{0}} \\
\nabla^{2} \mathbf{A}-\frac{1}{c^{2}} \frac{\partial^{2} \mathbf{A}}{\partial t^{2}}=-\frac{\mathbf{j}}{\varepsilon_{0} c^{2}}
\end{gathered}
$$

where these equations were obtained by choosing any gauge, known as the Lorenz gauge,

$$
\nabla \cdot \mathbf{A}=-\frac{1}{c^{2}} \frac{\partial \Phi}{\partial t} .
$$

Expressing $0.50,0.51$ and 0.52 in Minkowski space,

$$
\begin{gathered}
\partial^{\mu} \partial_{\mu} A^{\mu}=j^{\mu} \\
\partial_{\mu} A^{\mu}=0 .
\end{gathered}
$$

Because the components of the electric and magnetic field can be obtained via the vector potential, expressing this relationship in Minkowski space yields that the electric and magnetic field components are components of a 4 by 4 matrix known as the Faraday curvature tensor,

$$
F^{\mu \nu}=\partial^{\mu} A^{\nu}-\partial^{\nu} A^{\mu}=\left(\begin{array}{cccc}
0 & -E^{1} & -E^{2} & -E^{3} \\
E^{1} & 0 & -B^{3} & B^{2} \\
E^{2} & B^{3} & 0 & -B^{1} \\
E^{3} & -B^{2} & B^{1} & 0
\end{array}\right) \equiv\left(\begin{array}{cccc}
0 & -E_{x} & -E_{y} & -E_{z} \\
E_{x} & 0 & -B_{z} & B_{y} \\
E_{y} & B_{z} & 0 & -B_{x} \\
E_{z} & -B_{y} & B_{x} & 0
\end{array}\right) .
$$

Maxwell's equations can then be concisely written as,

$$
\begin{aligned}
\partial_{\mu} F^{\mu v} & =j^{\nu} \text { (Ampere's equation and electrostatics) } \\
\partial_{\mu} F^{\mu \nu} & =0 \text { (Faraday's equation and magnetostatics) }
\end{aligned}
$$

where $\mathrm{F}^{\mu v}$ is known as dual field-strength tensor and is defined as, 


$$
\mathrm{F}^{\mu v}=\frac{1}{2} \boldsymbol{\varepsilon}^{\mu v \alpha \beta} F_{\alpha \beta}
$$

where $\varepsilon^{\mu v a \beta}$ is called the totally antisymmetric fourth-rank tensor. If one takes the 4derivative of $\partial_{\mu} F^{\mu v}=j^{v}$ again, it can be shown that,

$$
\partial_{v}\left(\partial_{\mu} F^{\mu v}\right)=0
$$

implying that $\partial_{v} j^{v}=0$ which is the continuity equation and says that this current is conserved. This fundamentally ties gauge invariance to a conserved quantity, which by Noether's theorem, says that this gauge invariance is a symmetry of the system. 


\section{Appendix VI: Invariance of Schrödinger's Equation}

In quantum mechanics, the Schrödinger's equation is invariant to global phase changes,

$$
-\frac{\nabla^{2}}{2 m} \psi(\mathbf{r}, t)=i \frac{\partial \psi}{\partial t} \Leftrightarrow-\frac{\nabla^{2}}{2 m}\left\{e^{i \theta} \psi(\mathbf{r}, t)\right\}=i \frac{\partial \psi}{\partial t}\left\{e^{i \theta} \psi(\mathbf{r}, t)\right\} .
$$

This is actually a very fortunate fact because continuous symmetry operators can be represented as complex exponentials and their physical effects can be incorporated in the formation of the electron wave function. That is how an electron "knows" it is rotating, translating, or moving in time.

Something very interesting occurs in physics, when local phase change is imposed on Schrödinger's equation. First, local phase change means that the transformation involves a phase that also depends upon position,

$$
\psi(\mathbf{r}, t) \rightarrow \psi^{\prime}(\mathbf{r}, t)=e^{i q \Lambda(\mathbf{r}, t)} \psi(\mathbf{r}, t)
$$

When this is directly substituted into the Schrödinger equation,

$$
\begin{aligned}
\partial_{\mu} \psi^{\prime}(\mathbf{r}, t) & =\partial_{\mu}\left[e^{i q \Lambda(\mathbf{r}, t)} \psi(\mathbf{r}, t)\right] \\
& =e^{i q \Lambda(\mathbf{r}, t)}\left[\partial_{\mu} \psi(\mathbf{r}, t)+i q \psi(\mathbf{r}, t) \partial_{\mu} \Lambda(\mathbf{r}, t)\right]
\end{aligned}
$$

this shows that this is not just a phase change. Taking $\psi(\mathbf{r}, t) \rightarrow \psi^{\prime}(\mathbf{r}, t)$ in Schrödinger's equation,

$$
-\frac{\nabla^{2}}{2 m}\left\{e^{-i q \Lambda(\mathbf{r}, t)} \psi^{\prime}(\mathbf{r}, t)\right\}=i \frac{\partial}{\partial t}\left\{e^{-i q \Lambda(\mathbf{r}, t)} \psi^{\prime}(\mathbf{r}, t)\right\}
$$

and after some algebra,

$$
\frac{1}{2 m}(-i \nabla-q(\nabla \Lambda))^{2} \psi^{\prime}=\left(i \frac{\partial}{\partial t}+q \frac{\partial \Lambda}{\partial t}\right) \psi^{\prime}
$$


Equation 0.64 is not equal to,

$$
-\frac{\nabla^{2}}{2 m} \psi^{\prime}(\mathbf{r}, t)=i \frac{\partial \psi^{\prime}}{\partial t} .
$$

This means that local phase transformations are not invariant. It can be made invariant if the form of the derivative is modified,

$$
\partial_{\mu} \rightarrow D_{\mu}=\partial_{\mu}+i q A_{\mu}
$$

provided that $A_{\mu}$ also transforms as,

$$
A_{\mu} \rightarrow A_{\mu}-\frac{1}{q} \partial_{\mu} \Lambda
$$

then, if $\partial_{\mu} \psi$ is replaced with $D_{\mu} \psi$, the transformation of $D_{\mu} \psi$ will be,

$$
D_{\mu} \psi \rightarrow e^{i q \chi} D_{\mu} \psi
$$

which says that $D_{\mu} \psi$ transforms as $\psi$.

If one makes a comparison with the Schrödinger's equation in an electromagnetic field with the modified local gauge invariant Schrödinger's equation for a free particle,

$$
\left[\frac{1}{2 m}(-i \nabla-q \mathbf{A})^{2}+q \Phi\right] \psi=i \frac{\partial \psi}{\partial t} .
$$

If 0.61 is applied on 0.69 , then

$$
\left[\frac{1}{2 m}(-i \nabla-q(\mathbf{A}+\nabla \Lambda))^{2}+q\left(\Phi-\frac{\partial \Lambda}{\partial t}\right)\right] \psi^{\prime}=i \frac{\partial \psi^{\prime}}{\partial t}
$$

which is simply the statement that the potentials are gauge invariant. 


\section{Appendix VII: SEM Architecture}

The company that made this SEM is JEOL and its model is JSM-840. The column structure to the left in figure 4.1 is the actual column that houses the scanning electron beam. Figure 0.2 takes a closer look at the column section of the SEM.

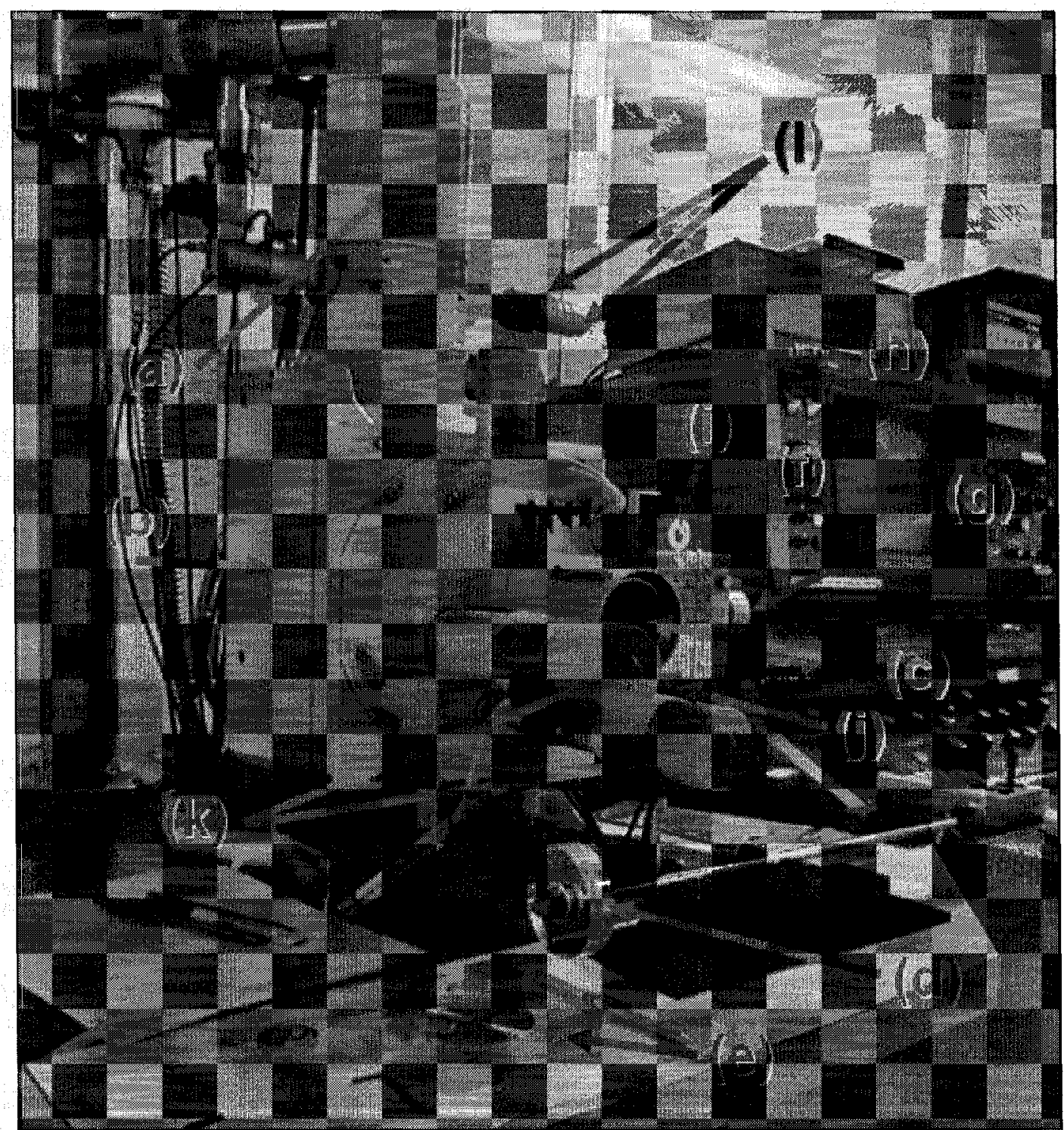

Figure 0.2. SEM Column Description. (a) column housing electron beam source and electron optics system of magnetic lens, (b) sample chamber, (c) airlock port, (d) plunger that goes in airlock to deliver or retrieve sample in chamber, (e) variety of sample holders, (f) airlock control button, (g) airlock door control, (h) vertical control of chamber platform (i.e. bring sample closer or farther from column-chamber opening - also called the gun), (i) roll control of chamber 
platform, $(j)$ yaw control of chamber platform and $(k)$ controls the xy motion of the chamber platform.

The collimated beam exits through an aperture from the column into a chamber holding the sample. The aperture is located between (a) and (b). Entrance to the chamber is obtained by access though the airlock (c). The sample holder plunger (d) is built to completely cover the airlock opening. A variety of sample holders (e) are available and can be screwed on to the airlock plunger. The airlock main switch (f) turns on or off the vacuum, and the airlock door (g) grants external access to the chamber.

The chamber platform can be raised or lowered towards the chamber-column aperture (also known as the gun) by using the knob (h). The platform roll angle is controlled by (i) and its yaw angle is controlled by (j). The platform $x y$-motion is controlled by (k). The beam angle through the aperture is controlled by (1). Figure 0.3 shows a close up of the control module. 


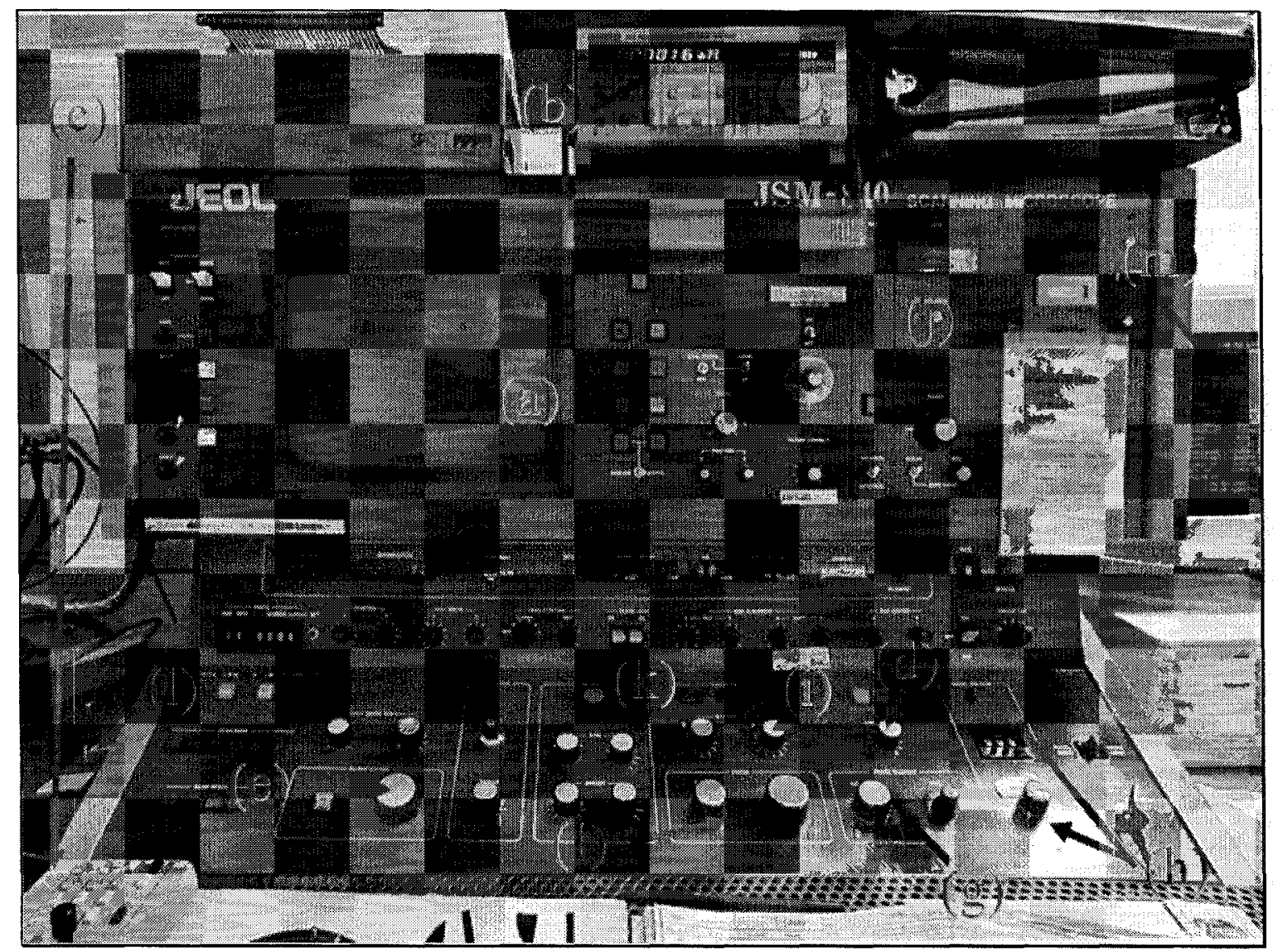

Figure 0.3. Close up of control module of the SEM. (a) CRT where imaging and writing can be seen, (b) emitted beam current meter, (c) stage controller and joystick, (d) wobble control of stage, (e) magnification control, (f) focus control, (g) beam current control, (h) voltage and current main switches, (i) stigmators, (j) beam blanker, (k) gun direction control, (l) SEM to computer control switch and (m) the computer used to control the SEM for writing.

The CRT, (a), is where the samples are viewed (when in microscope mode) and where the electron beam trace of what is being written is viewed (when in write mode). The beam current meter (b) is important when the beam is being conditioned to write. Later in this chapter it will be discussed how an appropriate beam current is selected, but for now, this is where the exact beam current is read. A processor unit and joystick, as indicated by (c) controls platform motion. Stage wobble control (d) determines whether the beam is hitting the sample surface in an orthogonal fashion. When (d) is activated, the image of the sample on the CRT will begin to fade in and out of view. If the beam is not 
perfectly normal to the surface, the image will move up and down or right and left. In order to correct this vertical or lateral motion, two dials (l) on the SEM column can be adjusted to fix the beam's inclination angle.

Magnification, controlled by (e), is very important when writing because it determines the field of view for writing structures. The focus is controlled by (f). This is important to make sure the beam is collimated. Beam diameter is controlled by the beam current (g). The main switches (h) for the voltage and current of the SEM control the absolute voltage and current reading for the SEM tungsten element. When in view mode, typical voltages are $25 \mathrm{kV}$ and when writing, it is taken up right to $39 \mathrm{kV}$ (the limit of this SEM). The stigmators (i) control the magnetic lens and adjust for astigmatism. The beam blanker (j) stops the beam from hitting the sample. The $x y$-motion of the aperture is controlled by $(\mathrm{k})$. When tuning these knobs, one will see the image become slightly brighter which means that all the energy was not previously on the sample. SEM viewing and writing modes are controlled by the switch (1), and (m) is the computer that controls the write software for the SEM.

Inside the column is where the electron optics occurs. Figure 0.4 shows the intricacies of the SEM column. The goal of this elaborate setup is to collect the electrons ejected from some given source material, shape the ejected electrons into a collimated beam, and then actively control the diameter of this collimated beam down into the column. 


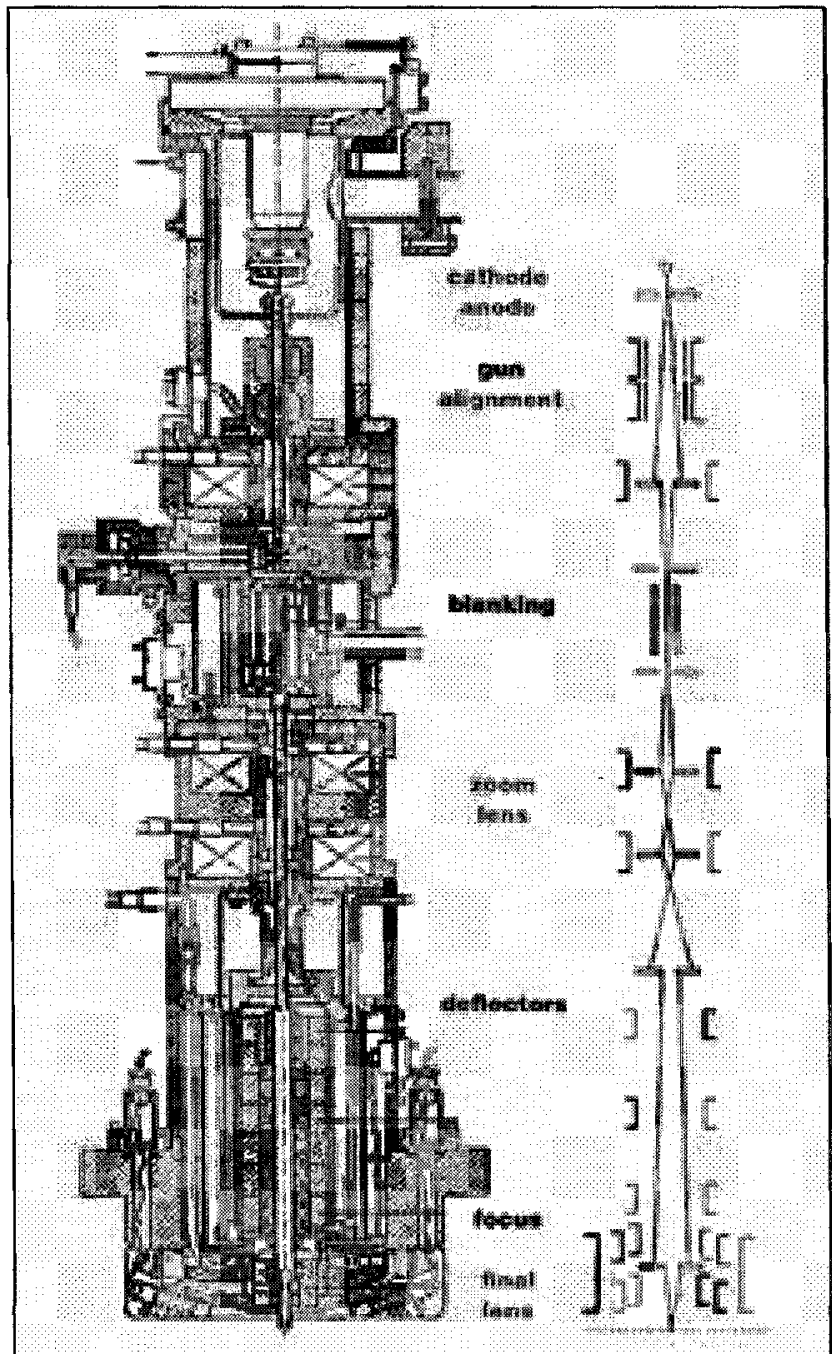

Figure 0.4. Physical and schematic drawing of electron beam column [65].

It is important to immediately acquire a small beam diameter in order for one to write small structures. This is called the virtual source diameter. The virtual beam diameter of two types of sources are shown in figure 0.5 . In the case with Carleton University's SEM, it has a tungsten source, which is the poorest type to have when doing EBL. It has the largest virtual beam diameter than all the other sources, which is about $25 \mu \mathrm{m}$.

The source should also have abundant brightness. Brightness is a parameter comparable to intensity. The higher the brightness, the more current there is in the 
electron beam. If the brightness is too low, then exposure rates in the resist may be inaccurate and lead to dull delineation between exposed and unexposed regions. Again,

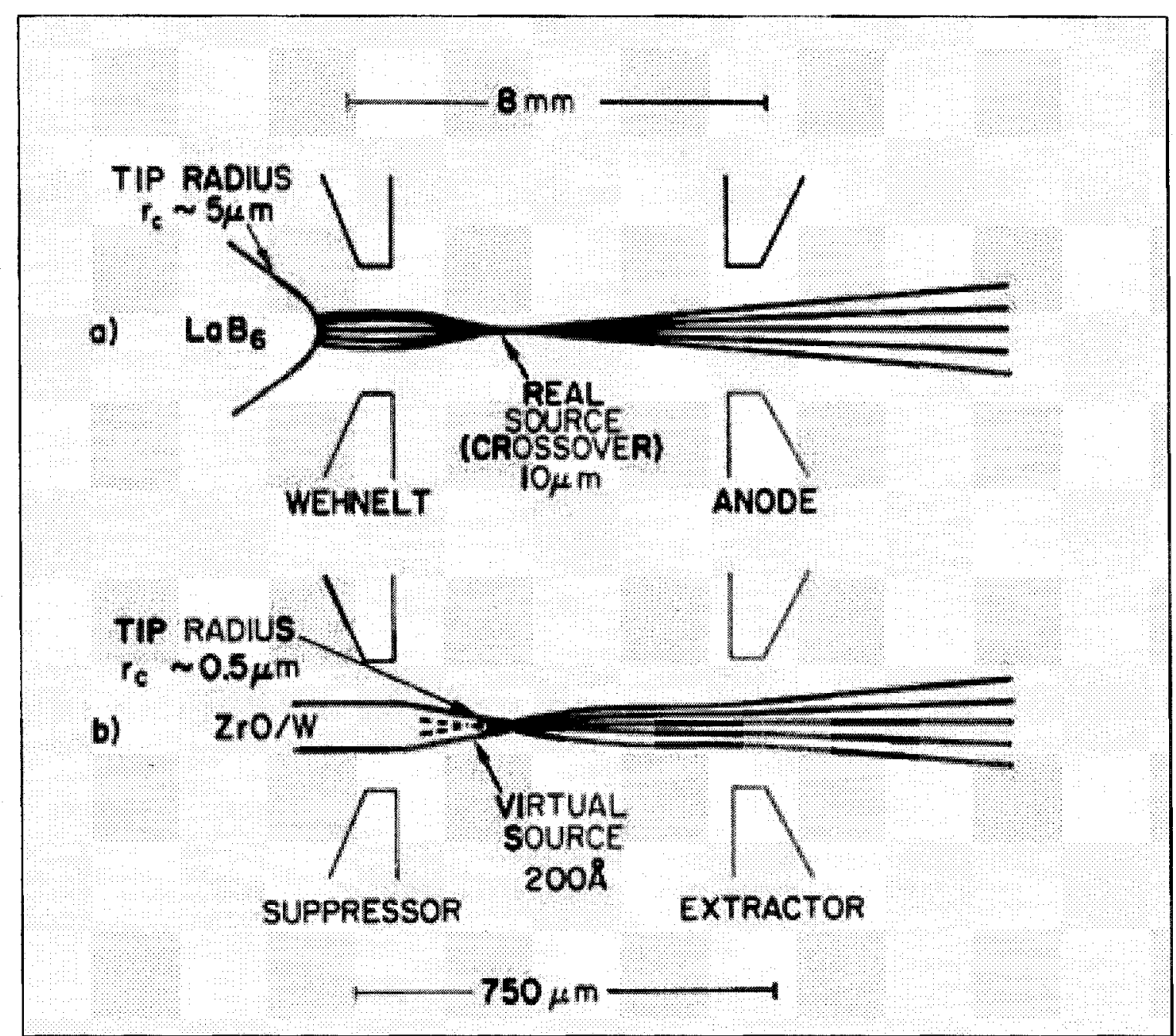

Figure 0.5. Virtual source diameter.

tungsten is the poorest of all the electron sources. The next important parameter in choosing an electron source is the energy spread. The wider the spread, the worse the beam is because a wide energy spread leads to chromatic dispersion. This chromatic dispersion is a result of the different energy electrons response to the magnetic field lens, resulting in a blurred exposure area. Once again, the tungsten source has one of the largest spreads. Table 0.1 shows the properties of some electron sources used in EBL systems. As one can see, tungsten is the lower end of quality. The reason that Carleton 
University's SEM has a tungsten source is that it has been primarily used as a microscope, so there has been no need to specialize.

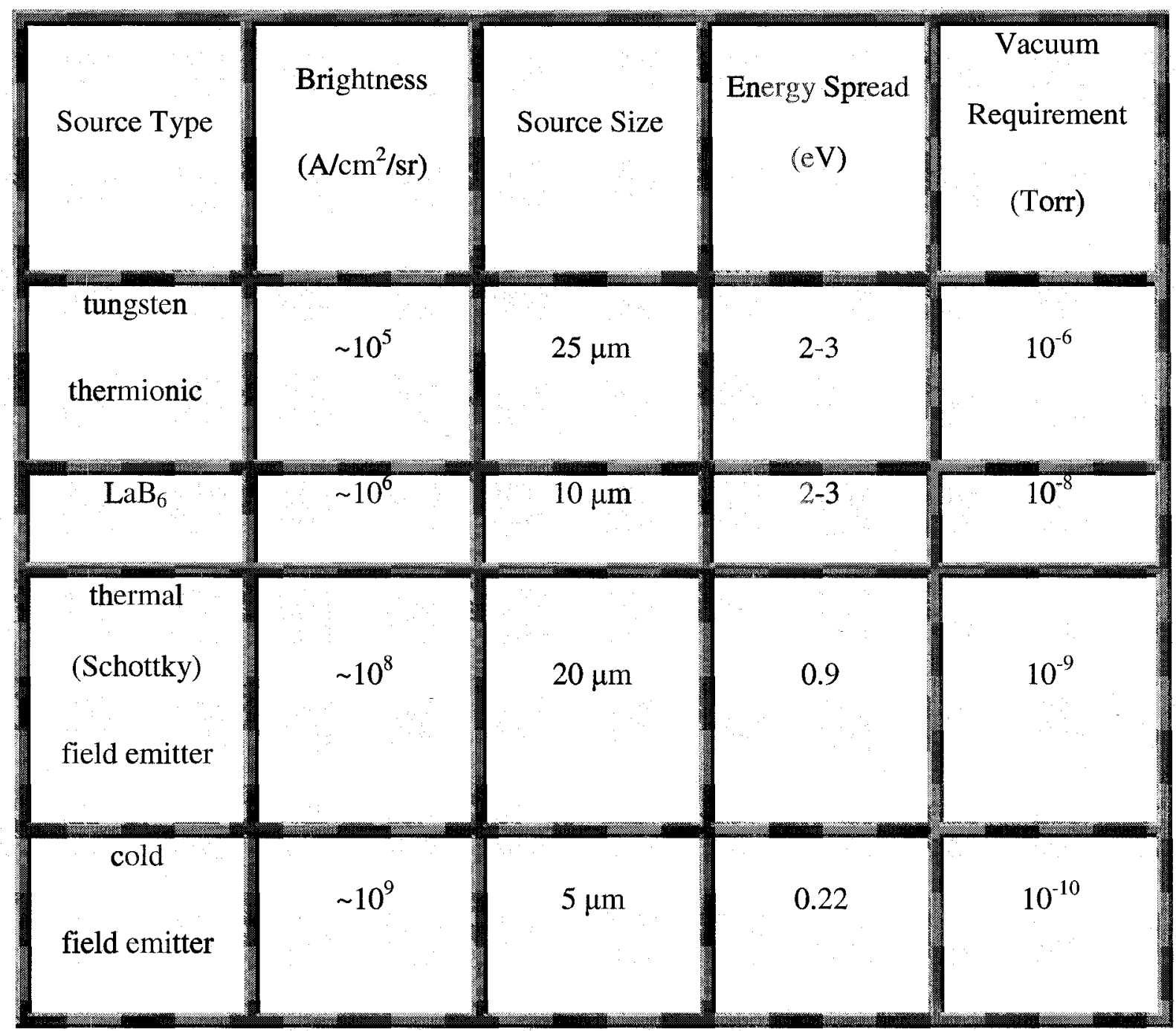

Table 0.1. Properties of electron sources used in EBL systems. 


\section{Appendix VIII: Applying Resist and Sample Preparation}

Figure 0.6 shows the spin station at Carleton University. This is where all resists for photolithography and EBL are applied. Figure 0.6(a) is the actual spin station where the samples are placed upon a chuck that has a vacuum hole that keeps the sample from flying off during spin. The sample holders range from 4 inch holders, 2 inch holders and holders for smaller samples. The spin parameters, such as spin speed and spin duration are controlled from (b). The container of ZEP is shown at (c). Near it is an eyedropper and Petri dish. When the sample is on the chuck, just before the spin begins, the resist is applied slowly via the eyedropper. As the resist is still being applied, the spinning should begin. The hexamethyldisilazane (HMDS) (d) which is a resist adhesion promoter, is then applied. HMDS must be applied before the ZEP. The hotplates, shown at (e) and (F), are used for baking the resist and HMDS. Lower temperatures are used for

photolithography resists and HMDS (i.e. $105^{\circ} \mathrm{C}$ ), while higher temperatures are used for the ZEP (i.e. $180^{\circ} \mathrm{C}$ ). 


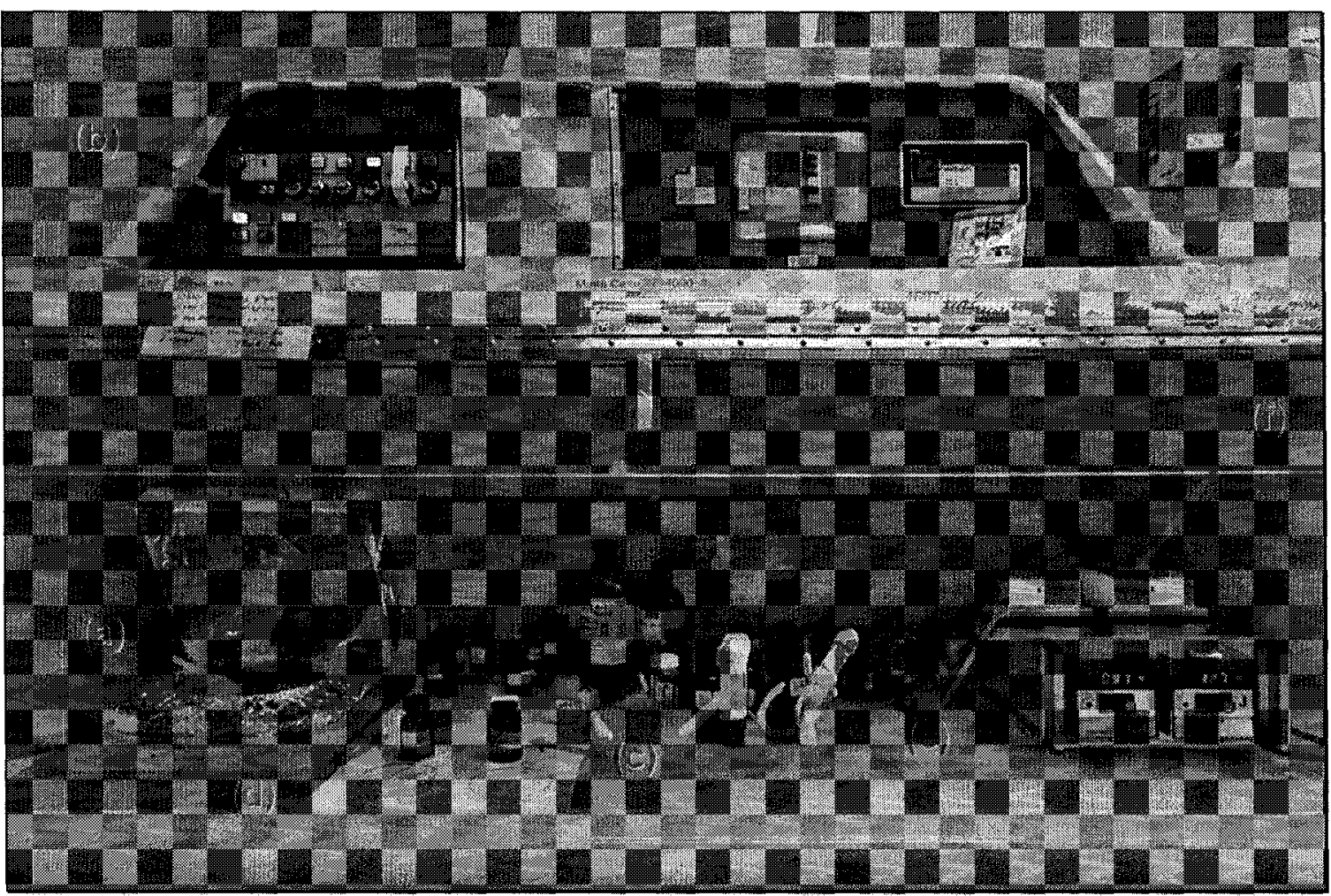

Figure 0.6. Resist spin station. (a) the spin area and spin chuck (not seen, inside), (b) control panel for spin station where spin rate and spin timing are controlled, (c) ZEP resist container, with eye droppers and petri dish near by, (d) hexamethyldisilazane (HMDS) which is applied on the substrate before any resist to promote adhesion, (e) hot plate set for low baking temperature - usually for photolithography resists and HMDS, and (f) higher temperature hotplate for ZEP.

Once the resist is applied and baked on, it is ready to be exposed. For EBL on a converted SEM, the procedure for loading a sample for viewing is the same procedure used to prepare it for EBL. The electron beam holder used for writing is important because different holders have different heights. This will slightly change the half-angle convergence beam described previously, which will affect the beam diameter. This means if one has found the right parameters for exposure on one sample holder, then one should stick to this sample holder when doing subsequent exposures. Once the sample has been placed on the holder it is ready to be loaded into the SEM. A small mark (scratch) is made on the sample to facilitate the SEM imaging of the sample and help 
orient them in space to know where to begin writing. It also allows the SEM user to focus on something to condition the beam for writing.

Figure 0.7 shows how one applies a scratch on the sample. The scratch is generally applied near the top of the sample so that the pattern can be written beneath it. After this, it is then ready to be loaded into the SEM. Once loaded, the SEM is powered up to the correct voltage and the source current is dialed up slowly. Once this is done, the SEM is put into view mode so that one can locate the scratch and condition the beam.

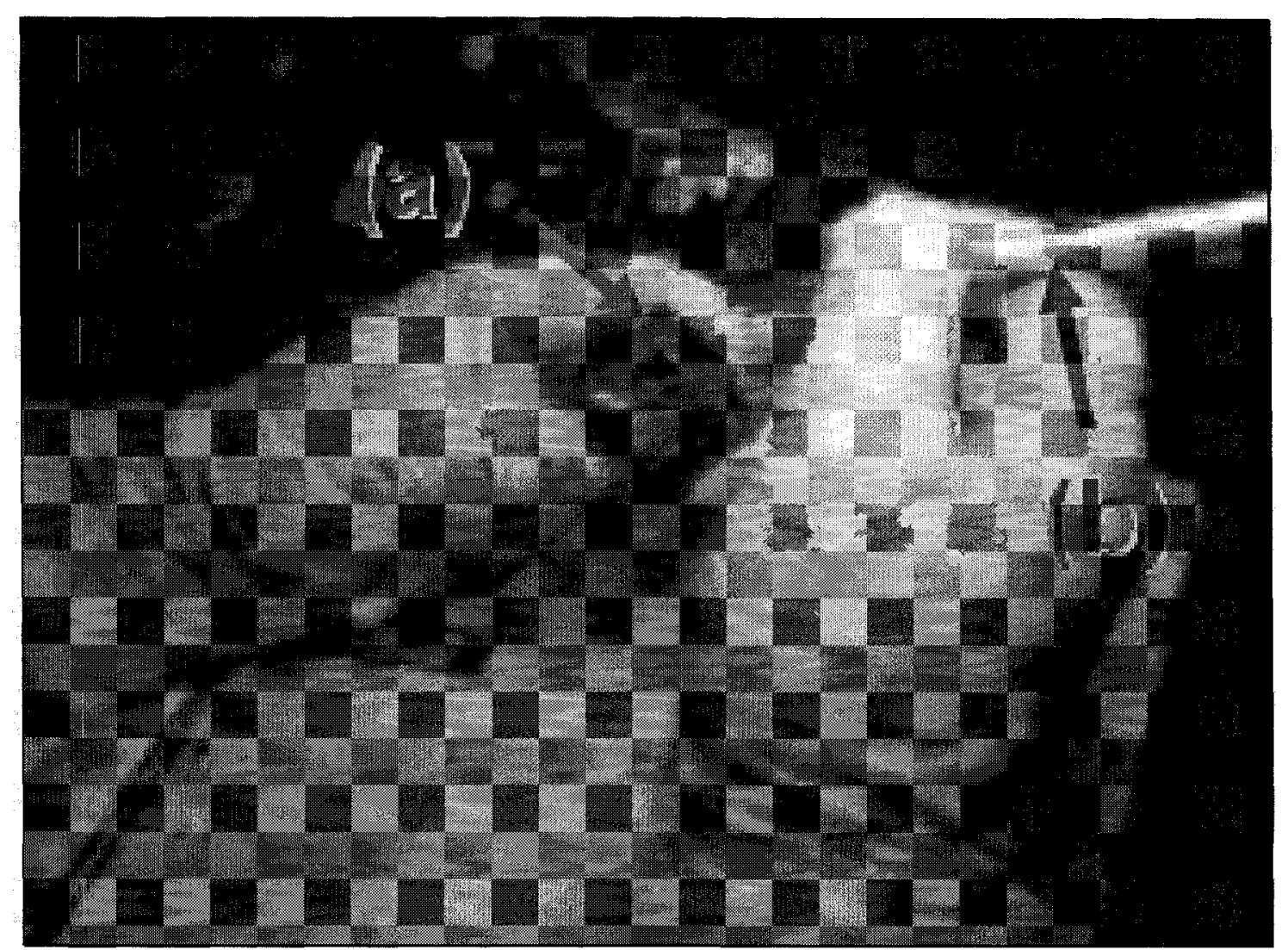

Figure 0.7. Applying scratch on sample before loading into SEM. (a) sample and holder and (b) diamond tipped scribe.

Once the beam is conditioned, the beam blanker can be aclivated which prevents the beam from hitting the surface, and the SEM can then be switched to write mode. 


\section{References}

[1] J.W.S. Rayleigh, "On the maintenance of vibrations by forces of double frequency and on the propagation of waves through a medium endowed with a periodic structure," Philos. Mag. 24, 145 (1887)

[2] H. Kogelnik et al., "Stimulated emission in a periodic structure," App. Phys. Lett. 18, 152 (1971)

[3] E. Yablonovitch, "Inhibited spontaneous emission in solid-state physics and electronics," Phys. Rev. Lett. 58, 2059 (1987)

[4] S. John, "Strong localization of photons in certain disordered dielectric Superlattices," Phys. Rev. Lett. 58, 2486 (1987)

[5] P.W. Anderson, "Absence of Diffusion in Certain Random Lattices," Phys. Rev. 109, $1492(1958)$

[6] S. Noda et al., "Trapping and emission of photons by a single defect in a photonic bandgap structure," Nature. 407, 608 (2000)

[7] D. Shechtman et al., "Metallic Phase with Long-Range Orientational Order and No Translational Symmetry," Phys. Rev. Lett. 53, 1951 (1984)

[8] Y. S. Chan et al., "Photonic Band Gaps in Two Dimensional Quasicrystals," Phys. Rev. Lett. 80, 956 (1998)

[9] Y. Wang et al., "Localized modes in defect-free dodecagonal quasiperiodic photonic crystals." Phys. Rev. B 68, 165106 (2003)

[10] K. Mnaymneh et al., "Mode localization and band-gap formation in defect-free photonic quasicrystals," Opt. Express 15, 5089 (2007)

[11] M. Kohmoto et al., "Localization in optics: quasiperiodic media," Phys. Rev. Lett. 58, $2436(1987)$

[12] W. Gellermann et al., "Localization of light waves in Fibonacci directly multilayers," Phys. Rev. Lett. 72, 633 (1994)

[13] C. Sibilia et al., "Electromagnetic properties of periodic and quasi-periodic onedimensional, metallo-dielectric photonic band gap structures," J. Opt. A: Pure Appl. Opt. 1, 490 (1999)

[14] A. Rostami et al., "Optical pulse distortion in Fibonacci-class quasi photonic crystals," IEEE Micro. Conf. Pro. 5, 4 (2005)

[15] K. B. Abdelaziz et al., "A broad omnidirectional reflection band obtained from deformed Fibonacci quais-periodic one dimensional photonic crystals," J. Opt. A: Pure Appl. Opt. 7544 (2005)

[16] G. G. Naumis et al., "Substitutional disorder in a Fibonacci chain: Resonant eigenstates and instability of the spectrum," Phys. Rev. B. 54, 15079, (1996)

[17] K. Kimura and S. Takeuchi, Quasicrystals: The State of the Art, World Scientific, Singapore (1991) 
[18] R. Penrose, "The role of aesthetics in pure and applied mathematical research," Bull. Inst. Math. Appl. 10, 266 (1074)

[19] S. S. M. Cheng et al., "Defect and transmission properties of two-dimensional quasiperiodic photonic band-gap systems," Phys. Rev. B 59, 4091 (1999)

[20] C. Jin et al., "Band gap and wave guiding effect in a quasiperiodic photonic crystal," Appl. Phys. Lett. 75, 1848 (1999)

[21] M. E. Zoorob et al., "Complete and absolute photonic bandgaps in highly symmetric photonic quasicrystals embedded in low refractive index materials," Mat. Sci. and Eng. 74, 168 (2000)

[22] M. E. Zoorob et al., "Complete photonic bandgaps in 12-fold symmetric quasicrystals," Nature 404, 740 (2000)

[23] M.A. Kaliteevski et al., "Two-dimensional Penrose-tiled photonic quasicrystals: from diffraction pattern to band structure," Nanotechnology 11, 274 (2000)

[24] M.A. Kaliteevski et al., "Two-dimensional Penrose-tiled photonic quasicrystals: diffraction of light and fractal density of modes," J. Mod. Opts. 47, 1771 (2000)

[25] C. Jin et al., "Two-dimensional dodecagonal and decagonal quasiperiodic photonic crystals in the microwave region," Phys. Rev. B. 61, 10762 (2000)

[26] M.A. Kaliteevski et al., "Diffraction and transmission of light in low-refractive index Penrose-tiled photonic quasicrystals," J. Phys: Condens. Matter 13, 10459 (2001)

[27] M.A. Kaliteevski et al., "The design of two-dimensional photonic quasicrystals by means of a Fourier transform method," J. Mod. Opts. 48, 9 (2001)

[28] S. David et al., "Isotropic Photonic Structures: Archimedean-Like tilings and quasicrystals," IEEE J. Quantum. Elec. 37, 1427 (2001)

[29] M. Bayindir et al., "Photonic band-gap effect, localization, and waveguiding in the two-dimensional Penrose lattice," Phys. Rev. B. 63, 161104 (2001)

[30] M. Hase et al., "Isotropic photonic band gap and anisotropic structures in transmission spectra of two-dimensional fivefold and eightfold symmetric quasiperiodic photonic crystals," Phys. Rev. B 66, 214205 (2002)

[31] B. P. Hiett et al., "Photonic band gaps in 12-fold symmetric quasicrystals," J. Mat. Sci. Elec. 14, 413 (2003)

[32] M. Notomi et al., "Lasing Action due to the two-dimensional quasiperiodicity of photonic quasicrystals with a Penrose lattice," Phys. Rev. Lett. 92, 123906 (2004)

[33] K. Nozaki et al., "Quasiperiodic photonic crystal microcavity lasers," Appl. Phys. Lett. 84, 4875 (2004)

[34] R. C. Gauthier et al., "Production of quasi-crystal template patterns using a dual beam multiple exposure technique," Opt. Express 12, 990 (2004)

[35] N. Horiuchi et al., "Isotropic photonic gaps in a circular photonic crystal," Opt. Lett. 29, 1084 (2004)

[36] J. Chaloupka et al., "Local density of states and modes of circular photonic crystal cavities," Phys. Rev. B. 72, 085122 (2005)

[37] A. Della Villa et al., "Band gap formation and multiple scattering in photonic quasicrystals with a Penrose-type lattice," 94, 183903 (2005)

[38] J. Romero-Vivas et al., "Photonic quasicrystals for application in WDM systems," Phys. Stat. Sol. 202, 997 (2005) 
[39] R. C. Gauthier et al., "Photonic band gap properties of 12-fold quasi-crystal determined through FDTD analysis," Opt. Express 13, 1985 (2005)

[40] K. Wang, "Light wave states in two-dimensional quasiperiodic media," Phys. Rev. B 73, $235122(2006)$

[41] R. C. Gauthier et al., "FDTD analysis of 12-fold photonic quasi-crystal central pattern localized states," Opt. Comm. 264, 78 (2006)

[42] A. Della Villa, "Localized modes in photonic quasicrystals with Penrose-type lattice," Opt. Express 14, 10021 (2006)

[43] Y. Lai et al., "Gap structures and wave functions of classical waves in large-sized two-dimensional quasiperiodic structures," Phys. Rev. B. 74, 054305 (2006)

[44] Y. Wang, "Coupled-resonator optical waveguides in photonic crystals with Archimedean-like tilings," Europhs. Lett., 74, 261 (2006)

[45] R. C. Gauthier, "FDTD analysis of out of plane propagation in 12-fold photonic quasi-crystals," Opt. Comm. 269, 395 (2007)

[46] N. W. Ashcroft and N. D. Mermin, Solid State Physics, Harcourt College, New York (1976)

[47] M. E. Peskin and D. V. Schroeder, An Introduction to Quantum Field Theory, Addison-Wesley, New York (1998)

[48] D. M. Sullivan, Electromagnetic Simulation Using the FDTD Method, Wiley-IEEE Press, New Jersey (2000)

[49] F. W. Byron and R. W. Fuller, Mathematics of Classical and Quantum Physics, Dover Publications, New York (1970)

[50] J. Jin, Finite Element Method for Electromagnetics, IEEE, Toronto (2003)

[51] J. D. Joannopoulos, Robert D. Meade and Joshua N. Winn, Photonic Crystals: Molding the Flow of Light, Princeton University Press, Singapore (1995)

[52] J. Nabity, http://www.jcnabity.com/

[53] D. W. Prather, "High-efficiency coupling structure for a single-line-defect photoniccrystal waveguide," Opt. Lett. 27, 1601 (2002)

[54] Agilent 81600B Tunable Laser Source Family, Technical Specifications, May 2006

[55] D. Sutter et al., "Ultrasonic investigation of phononic Penrose crystals," Phys. Stat. Sol. 1, 2716 (2004)

[56] L. Marrucci et al., "Optical spin-to-orbital angular momentum conversion in inhomogeneous anisotropic media," Phys. Rev. Lett. 96, 163905 (2006)

[57] E. Waks et al., "Quantum networking with quantum dots coupled to micro-cavities," Proc. SPIE 6780, 67800A (2007)

[58] S. Feng et al., "Why is the orbitial angular momentuym conserved in spontaneous parametirc down-conversion?," Proc. SPIE 6710, 67100C (2007)

[59] C. I. Osorio et al., "The orbital angular momentum spectrum of photons generated via parametric down-conversion," Proc. SPIE 6710, 67100D (2007)

[60] R. Lifshitz et al., "Photonic quasicrystals for nonlinear optical frequency conversion," Phys. Rev. Lett. 95, 133901 (2005)

[61] M. Notomi et al., "Lasing Action due to the two-dimensional quasiperiodicity of photonic quasicrystals with a Penrose lattice," Phys. Rev. Lett. 92, 123906 (2004)

[62] M. Charlton et al., "Photonic quasi-crystal LEDs: design, modeling, and optimization,” Proc. SPIE 6486, 64860R1 (2007) 
[63] J. Romo-Vivas et al., "Resonant add-drop filter based on a photonic quasicrystal," Opt. Express 13, 826 (2005)

[64] M. Bayindir et al., "Photonic band gap and localization in two-dimensional Penrose lattice," Lasers and Electro-Optics CLEO, 596 (2001)

[65] P. Rai-Choudhury, Handbook of Microlithography, Micromachining, and Microfabrication. SPIE Publications, New York (1997) 